Friedhelm Herb

Marktwirtschaftliche Innovationspolitik 


\section{Friedhelm Herb}

\section{Marktwirtschaftliche Innovationspolitik}

Geringe Wachstumsraten und hohe Arbeitslosigkeit haben die Innovationspolitik in das Zentrum der politischen Diskussion gerückt. In dieser Arbeit werden die gesamt- und einzelwirtschaftlichen Grundlagen der Innovationstätigkeit analysiert. Eingehend werden der Einfluß der Wettbewerbsbedingungen auf das Innovationsverhalten sowie die Bedeutung der steuerlichen Institutionen und der Finanzierungsmöglichkeiten für die Risiko- und Innovationsbereitschaft untersucht. Darauf aufbauend wird das Konzept einer akkommodierenden Innovationspolitik entwickelt, das der gegenwärtig praktizierten, induzierend angelegten Politik gegenübergestellt wird.

Friedhelm Herb wurde 1961 in Heilbronn geboren. Studium der Wirtschaftswissenschaften von 1980-1985 an der Universität Hohenheim. Von 1985-1988 Wissenschaftlicher Mitarbeiter bei Prof. Dr. Cay Folkers am Lehrstuhl für Finanzwissenschaft im Institut für Volkswirtschaftslehre der Universität Hohenheim. 1986-1988 Stipendiat nach dem Landesgraduiertenförderungsgesetz Baden-Württemberg. 
Marktwirtschaftliche Innovationspolitik 


\section{Hohenheimer \\ Volkswirtschaftliche Schriften}

Herausgegeben von

Prof. Dr. Cay Folkers, Prof. Dr. Klaus Herdzina,

Prof. Dr.Franz Mehler t, Prof. Dr. Walter Plesch,

Prof.Dr.Ingo Schmidt, Prof.Dr. Helmut Walter,

Prof.Dr. Josua Werner

Band 9

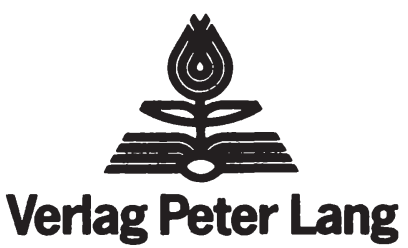

Frankfurt am Main · Bern · New York · Paris 


\section{Friedhelm Herb}

\section{Marktwirtschaftliche Innovationspolitik}

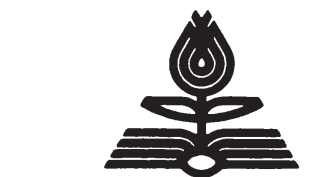

\section{Verlag Peter Lang}

Frankfurt am Main - Bern - New York - Paris 
CIP-Titelaufnahme der Deutschen Bibliothek

Herb, Friedhelm:

Marktwirtschaftliche Innovationspolitik / Friedhelm Herb. Frankfurt am Main ; Bern ; New York ; Paris : Lang, 1988

(Hohenheimer volkswirtschaftliche Schriften ; Bd. 9)

ISBN 3-631-40752-1

NE: GT

Open Access: The online version of this publication is published on www.peterlang.com and www.econstor.eu under the international Creative Commons License CC-BY 4.0. Learn more on how you can use and share this work: http://creativecommons. org/licenses/by/4.0.

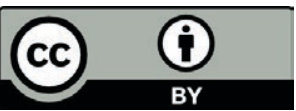

This book is available Open Access thanks to the kind support of ZBW - Leibniz-Informationszentrum Wirtschaft.

\author{
D 100 \\ ISSN 0721-3085 \\ ISBN 3-631-40752-1 \\ ISBN 978-3-631-75475-7 (eBook) \\ (c) Verlag Peter Lang GmbH, Frankfurt am Main 1988 \\ Alle Rechte vorbehalten.
}

Das Werk einschließlich aller seiner Teile ist urheberrechtlich geschützt. Jede Verwertung außerhalb der engen Grenzen des Urheberrechtsgesetzes ist ohne Zustimmung des Verlages unzulässig und strafbar. Das gilt insbesondere für Vervielfältigungen, Übersetzungen, Mikroverfilmungen und die Einspeicherung und Verarbeitung in elektronischen Systemen.

Printed in Germany 
When the dragons grow too mighty to slay with pen or sword, I grow weary of the battle and the storm I walk toward. When all around is madness and there's no safe port in view, I long to turn my path homeward to stop awhile with you.

(Neil Pert) 
Friedhelm Herb - 978-3-631-75475-7 Downloaded from PubFactory at 01/11/2019 04:33:39AM via free access 


\section{VORWORT}

Die vorliegende Arbeit entstand während meiner Tätigkeit als wissenschaftlicher Mitarbeiter bei Prof. Dr. Cay Folkers am Lehrstuhl für Finanzwissenschaft der Universität Hohenheim. Herr Prof. Dr. C. Folkers hat diese Arbeit angeregt und begleitet; ihm sei an erster stelle gedankt.

Meinen Kollegen Dipl. oec. Thomas Märtz und Dipl.-Volkswirt Peter Mendler möchte ich für ihre stete Diskussionsbereitschaft und für zahlreiche Hinweise danken, die mir in verschiedenen Flauten zu neuem Rückenwind verhalfen.

Bei den Diplom-okonomen Andreas Maurer, Jürgen Menges und Sabine Reichelt möchte ich mich ebenfalls für die Unterstützung bedanken.

Besonderen Dank schulde ich dem Land Baden-Württemberg für ein Stipendium nach dem Landesgraduiertenförderungsgesetz, das mir die Möglichkeit gab, mich auf mein Promotionsvorhaben zu konzentrieren.

\footnotetext{
(0) Die veröfentlichung dieser Arbeit erfolgte mit freundicher Unterstützung der LG-stiftung: Ausbilseltung dung, Fort- und Weiterbildung, einer stiftung der Landesgirokasse stuttgart. Für die großzügige Druckkostenbeihilfe bedanke ich mich herzlich.
}

Hohenheim, im Juli 1988

Friedhelm Herb 
Friedhelm Herb - 978-3-631-75475-7 Downloaded from PubFactory at 01/11/2019 04:33:39AM via free access 


\section{GLIEDERUNG}

A.

EINLEITUNG

S. 1

I. PROBLEMSTELLUNG

S. 1

II. GANG DER UNTERSUCHUNG

S. 3

TEIL 1: GRUNDLEGENDE GESAMT- UND

EINZELWIRTSCHAFTLICHE ZUSAMMENHTNGE

S. 6

B. WIRTSCHAFTSWACHSTUM UND

$\begin{array}{ll}\text { PRODUKTIVITRTSENTWICKLUNG } & \text { S. } 6\end{array}$

I. KOMPONENTEN DES WIRTSCHAFTSWACHSTUMS

S. 6

II.

DETERMINANTEN DER PRODURTIVITATSENTWICKLUNG

S. 7

1. Prozeßinnovationen und Economies of Scale

S. 7

2. Produktivitätsverluste durch suboptimale

Faktorallokation

S. 11

2.1 Produktivitätsdeterminanten der Inputfaktoren Kapital und Arbeit.

S. 12

2.1.1 Faktorwanderung und Faktormobilität

S. 12

2.1.2 Faktorpreisentwicklung und

Faktorsubstitutionsmöglichkeiten

S. 13

2.1.3 Faktorqualität

S. 14

2.1.3.1 Bestimmungsgrößen der Qualität der Arbeitsleistung

S. 14

2.1.3.2 Bestimmungsgrößen der qualităt des Sachkapitals

S. 15

2.1.4 Leistungsintensität der eingesetzten Faktoren

S. 16

2.1.4.1 Bestimmungsgrößen der Leistungsintensität des Arbeitseinsatzes

S. 16

2.1.4.2 Bestimmungsgrößen der Leistungsintensität des eingesetzten Sachkapitals

S. 18

2.2 Institutionelle Determinanten der

Produktivitätsentwicklung

s. 18 
2.2.1 Regulierung von Ausnahmebereichen

S. 18

2.2.2 Wettbewerbs-, Handels- und Patentpolitik

S . 20

2.2.3 Regulierung intangibler Inputfaktoren

S. 21

2.2 .4

Besteuerung

S. 21

2.2 .5

Dienstleistu

S. 22

2.2 .6 Rent-Seeking

S. 23

2.2 .7

Sonstige institutionelle Faktoren

S. 23

3.

Gesamtwirtschaftliche Determinanten der

Produktivitätsentwicklung

S . 24

3.1 Auslastungsgrad der Produktionsfaktoren

S. 24

3.2

Inflation

S. 25

III. ERGEBNISSE EMPIRISCHER FORSCHUNG ZUR PRODURTIVITATSENTWICKLUNG

S. 26

1. Wirtschaftswachstum und

Produktivitätsentwicklung

S. $\quad 27$

2. Anteile von Inputsteigerungen und

Produktivitätserhöhungen am Wachstum

des Sozialprodukts

S. 30

3. Romponenten des Wirtschaftswachstums

aufgrund erhöhten Faktoreinsatzes

S. 30

4. Komponenten der Produktivitätsentwicklung

S. 31

IV. ZUSAMMENFASSUNG

S. 41

C.

DETERMINANTEN VON INNOVATIONSENTSCHEIDUNGEN

S. 43

I.

DAS NEORLASSISCHE INVESTITIONSMODELL

S. 43

1 .

Das Grundmodell

S. 43

2 .

Die wirkungen der Besteuerung im

neoklassischen Investitionsmodel1

S. 46

3.

Grenzen des neoklassischen Investitionsmodells

S. 48

II.

DER INNOVATIONSPROZESS

S. 50

1 .

Technology-push und Demand-pull

S. 50

2.

Der Ablauf von Innovationsprozessen

S. 52

3.

Der Innovationsablauf unter Berücksichtigung

von Ronkurrenzbeziehungen

S. 54 
III. DIE BESONDERHEITEN VON INNOVATIONSENTSCHEIDUNGEN

S. 56

IV. ZUSAMMENFASSUNG

S. 57

D. DER EINFLUSS DER MARKTVERHXLTNISSE

$\begin{array}{ll}\text { AUF DIE INNOVATIONSBEREITSCHAFT } & \text { S. } 58\end{array}$

I. DIE INNOVATIONSPLANUNG OHNE

KONKURRENZBEZIEHUNGEN

S. 58

1. Die Innovationskosten

S. 58

2. Der Innovationskalkül

S. 60

II. DIE INNOVATIONSENTSCHEIDUNG UNTER WETTBEWERBSBEDINGUNGEN S. 63

1. Der entscheidungstheoretische Ansatz $\quad 63$

1.1 Das Modell S. 63

1.2 Zusammenfassung $\quad$ s. 72

2. Der spieltheoretische Ansatz $\quad$ S. 73

2.1 Das Modell S. 73

2.2 Zusammenfassung $\quad$ S. 78

3. Ergänzungen $\quad$ S 79

III. WOHLFAHRTSWIRKUNGEN $\quad$ S. 80

IV. ZUSAMMENFASSUNG $\quad$ S. 82

TEIL 2: DER EINFLUSS VON STEUERN UND FINANZIERUNGSBEDINGUNGEN AUF DIE RISIKO- UND INNOVATIONSBEREITSCHAFT

B. DER EINFLUSS DER BESTEUERUNG AUF

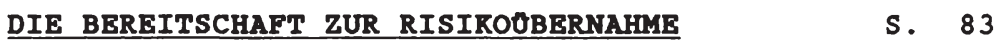

I. DAS PORTEFEUILLE-MODELL $\quad S .84$

1. Die Modellgrundlagen $\quad$ S. 84 
2. Die sichere Anlageform erbringt keinen Ertrag $(r=0) \quad$ S. 86

3. Die sichere Anlageform erbringt Ertrag $(r>0) \quad$ S. 88

II. DIRERTER ANSATZ S 92

1. Der Modellansatz $\quad$ S. 92

2. Proportionale Besteuerung $\quad$ S. 96

3. Progressive Besteuerung $\quad$ S. 97

4. Die Wirkungen von Tarifreformen auf die $\begin{array}{ll}\text { Risikobereitschaft } & \text { S. } 98\end{array}$

III. UNVOLLSTANDIGER VERLUSTAUSGLEICH

IV. DIE BEHANDLUNG SCHWANKENDER EINKOMMEN S. 101

V. DIE BEDEUTUNG DER STEUERBILANZPOLITIR S. 103 VI. DIE WIRKUNG DER BESTEUERUNG VON VERMOGEN
UND UMSATZ AUF DIE RISIROBEREITSCHAFT

VII. DIE DISKRIMINIERUNG UNTERNEHMERISCHER TATIGREIT $\quad$ S. 112

VIII. DAS STEUERPOLITISCHE RISIKO S. 113

IX. ZUSAMMENFASSUNG S. 114

F. DER EINFLUSS DER FINANZIBRUNGSBEDINGUNGEN AUF DIE INNOVATIONSBEREITSCHAFT 116

$\begin{array}{ll}\text { I. RAPITALBILDUNG } & \text { S. } 117\end{array}$

1. Die steuerlichen Rahmenbedingungen der Rapitalbildung in privaten Haushalten S. 117

2. Die Förderung bestimmter Vermögensanlagen $\quad$. 118

3. Die Rapitalbildung in Unternehmen $\quad$ S. 119 
II. RAPITALBESCHAFFUNG

S. 120

1. Die steuerliche Behandlung alternativer

Finanzierungsformen

S. 120

1.1 Die steuerlichen Implikationen der einzelnen Finanzierungsformen

S. 120

1.1.1 Fremdinanzierung

S. 120

1.1.2 Beteiligungsfinanzierung

S. 121

1.1.3 Selbstfinanzierung

S. 123

1.2 Vergleich der Finanzierungsformen

S. 123

1.2.1 Fremdfinanzierung versus

Beteiligungsfinanzierung

S. 123

1.2.2 Selbstfinanzierung versus

Beteiligungsfinanzierung

S. 126

1.2 .3

Selbstfinanzierung versus Fremdfinanzierung

S. 127

1.3

Der Einfluß der Finanzierungsbedingungen

auf die Investitionsplanung

S. 128

1.4 Fazit

S. 129

2. Die Finanzierung von Innovationsprojekten

S. 130

2.1

Problemstellung

S. 130

2.2 Fremdfinanzierung

S. 131

2.2.1 Fremdkapitalaufnahme bei Kreditinstituten

S. 131

2.2.2 Fremdfinanzierung mit schuldscheindarlehen und Industrieobligationen

S. 136

2.2.3 Finanzierung durch Pensionsrückstellungen

S. 137

2.2.4 Zusammenfassung

S. 137

2.3 Eigenfinanzierung

S. 138

2.3.1 Eigenfinanzierung und Rechtsform

S. 138

2.3.2 Zusammenfassende Gegenüberstellung

S. 141

3. Die Eigenfinanzierung von Unternehmen

über den Kapitalmarkt

S. 144

3.1 Die steuerlichen Folgen der Rechtsformwahl

S. 144

3.1 .1

Die steuerlichen Folgen des Rechtsformwechsels

S. 144

3.1 .2

Die Belastungen bei der Aktiengesellschaft

S. 146

3.2

Die Bedingungen für die Börsenzulassung

S. 146

4.

Finanzierung durch nicht-gesellschafts-

rechtliche Beteiligungstitel

S. 147

III. ZUSAMMENFASSUNG

S. 148 
TEIL 3: MARKTWIRTSCHAFTLICHE INNOVATIONSPOLITIR:

MOGLICHKEITEN - GRENZEN - RONZEPTE

S. 151

G. MOGLICHREITEN UND GRENZEN DER

INNOVATIONSPOLITIR

S. 151

I. GEGENSTAND DER INNOVATIONSPOLITIR

S. 151

II. VORAUSSETZUNGEN FUR EINE INNOVATIONSPOLITIR S. 152

1. Notwendige Bedingung

S. 152

1.1 Optimalitätsbedingungen

S. 152

1.2 Die Informationserfordernisse

S. 155

1.3

Spezielle Probleme der Innovationspolitik

S. 160

2 .

Hinreichende Bedingung

S. 162

2.1 Rapitalisierungseffekte

S. 162

2.1.1 Die Rapitalisierung im Marktproze $\beta$

S. 163

2.1 .2

Die Kapitalisierung im politischen Proze $\beta$

S. 164

2.2

Die Interventionsakzeleration

S. 166

III. MOGLICHREITEN DER INNOVATIONSPOLITIR

S. 167

IV. GRUNDLAGEN EINER

ARROMMODIERENDEN INNOVATIONSPOLITIK

S. 169

1. Die Funktionen von Unternehmen und Stat in einer marktwirtschaftlichen ordnung

S. 169

2. Grundzüge einer

akkommodierenden Innovationspolitik

S. 170

V. ZUSAMMENFASSUNG

S. 176

H. INNOVATIONSPOLITIK IN DER

BUNDESREPUBLIK DEUTSCHLAND

S. 177

I. ZIELE UND GRUNDSATZE

S. 177

II. ORONOMISCHE BEGRONDUNGEN DER

FORSCHUNGS- UND TECHNOLOGIEPOLITIR

IN DER BUNDESREPUBLIK DEUTSCHLAND

S. 179

1. Externalitäten

S. 179 


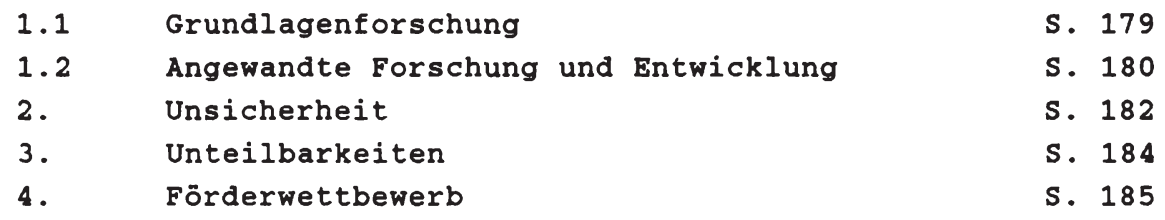

III. INNOVATIONSPOLITIK IN DER BUNDESREPUBLIK DEUTSCHLAND - EINE RRITISCHE BESTANDSAUFNAHME S. 191

1. Die Ausgaben für Forschung und Entwicklung S. 191

2. Die Steuerpolitik S. 199

3. Die Kapitalmarktpolitik S. 203

4. Die Wettbewerbspolitik 206

$\begin{array}{lr}\text { IV. FAZIT } & \text { S. } 207\end{array}$

V. VORSCHLAGE FUR MASSNAHMEN IM RAHMEN EINER ARKOMMODIERENDEN INNOVATIONSPOLITIK S. 209

1. Maßnahmen im Bereich der steuerpolitik S. 210

2. Maßnahmen im Bereich der Kapitalmarktpolitik S. 213

3. Maßnahmen im Bereich der Industriepolitik s. 214

4. Implementationsprobleme $\quad$ S. 215

$\begin{array}{ll}\text { J. ZUSAMMENFASSUNG } & \text { S. } 216\end{array}$

$\begin{array}{ll}\text { LITERATURVERZEICHNIS } & \text { S. } 227\end{array}$ 
Friedhelm Herb - 978-3-631-75475-7 Downloaded from PubFactory at 01/11/2019 04:33:39AM via free access 


\section{$\underline{I}$} PROBLEMSTELLUNG

Die Sozialproduktsentwicklung ist seit der Mitte der siebziger Jahre in den westlichen Industrienationen durch einen auffallenden Rückgang der Wachstumsraten gekennzeichnet. Zu Beginn der achtziger Jahre setzte zwar eine stabilisierung der Wachstumsraten ein, jedoch auf niedrigem Niveau'). Die mit dieser Entwicklung einhergehenden gravierenden Beschãftigungsprobleme, verstärkt durch die demographische Entwicklung, ließen den Ruf nach einer "Neuen Industriepolitik" oder auch "Neuen Industrie- und Forschungspolitik" laut werden'). Mit einer solchen neuen strategie sollen zwei ziele erreicht werden. Zum einen soll der verlust von Arbeitsplätzen durch die Konkurrenz der schwellenländer in traditionellen Industriezweigen gestoppt werden. Zum zweiten sollen durch die Förderung "zukunftsträchtiger Industrien" neue Märkte erschlossen werden ${ }^{3}$ '.

Gegenstand dieser Arbeit sind die zur Erreichung des letztgenannten zieles durchgeführten und geforderten Maßnahmen. Untersucht werden die Voraussetzungen für eine zielgerichtete Innovationspolitik sowie die Möglichkeiten, die den politischen Entscheidungsträgern zur Gestaltung der innovativen Aktivitäten der privaten Wirtschaftssubjekte zur Verfügung stehen.

Auffällig ist in diesem Bereich der Wirtschaftspolitik die sich ständig ausweitende Anzahl von Förder- und Aktionsprogrammen für immer neue Bereiche mit immer detaillierteren Regelungen. Einschlägige Maßnahmen werden parallel auf supranationaler, Bundes-, Landes- und kommunaler Ebene initiiert' 1 .

1) Vgl. Tabelle B.I

2) Vgl. Schmidt. K.-D. (1985), S. 63 und Issing, 0. (1986), S. 2

3) Vgl. Schmidt, K.-D. (1985), S. $63 \mathrm{ff}$

4) Vgl. Dittes, E. (1987), Bundesminister für Forschung und Technologie (1986) und Ministerium für Wirtschaft, Mittelstand und Technologie Baden-Württemberg (1987) 
Die Analyse dieses "technologiepolitischen Aktivismus"1) erfordert neben der Untersuchung der ökonomischen Grundlagen einer solchen Politik auch die Einbeziehung politisch-ökonomischer Prozesse. Durch diese Erweiterung um eine positive politisch-ökonomische Analyse der Förderaktivitäten ändert sich die für die Operationalität innovationspolitischer Maßnahmen entscheidende Fragestellung. Um den staatlichen Handlungsbedarf im Bereich der Innovationspolitik zu bestimmen, werden nicht aus ökonomischer sicht ideale Anreizstrukturen den bestehenden, unvollkommenen institutionellen Regelungen gegenübergestellt ("Nirvana Approach"2)). Vielmehr werden die Möglichkeiten der Innovationspolitik beurteilt, indem die Effizienz staatlicher Maßnahmen innerhalb des existierenden ökonomischen und politischen systems analysiert wird. Dementsprechend stellt diese Arbeit auf Verbesserungen innerhalb des gegebenen politisch-ökonomischen systems ab.

Der in dieser Arbeit verwendete Innovationsbegriff geht auf eine von J.A. Schumpeter getroffene Definition zurück. Schumpeter beschreibt die Innovationstätigkeit als "Doing things differently."3) Eine Innovation ist somit nicht nur die Durchsetzung technischer Neuerungen sondern jede wirtschaftliche Verwertung neuartiger Kombinationen von Angebot und Nachfrage, seien es neue Produkte oder Produktqualitãten, Produktionsverfahren oder Organisationsformen').

wird in dieser Arbeit von Innovationstätigkeit gesprochen, werden alle für ein Unternehmen neuartigen Kombinationen eingeschlossen. Für den Innovationsbegriff wird also nicht darauf abgestellt, ob es sich um die erste kommerzielle Nutzung einer neuartigen Kombination in der Volkswirtschaft überhaupt handelt, sondern ob sie für das betreffende Unternehmen eine Neuerung darstellt( ) . Dieser einzelwirtschaftliche Innovationsbegriff erscheint im Rahmen der vorliegenden Arbeit sinn-

1) Staudt, E. (1985), S. 646

2) Demsetz, H. (1969), S. 1

3) Schumpeter, J.A. (1939), S. 84; vgl. dazu die deutsche Obersetzung dieser Definition: Innovation heißt "Andersmachen"; Schumpeter, J.A. (1961), S. 91.

4) Vgl.Schumpeter, J.A. (1964), S. $100 f$

5) Vgl. Mohr, H. -W. (1977), S. 23 
voll, da die Innovationspolitik nicht nur auf aus volkswirtschaftlicher sicht neuartige Kombinationen abstellt, sondern auf die Neuerungsaktivitäten der einzelnen Unternehmen abzielt.

Die Analyse dieser Arbeit hebt auf die Möglichkeiten der Beeinflussung von Innovationsentscheidungen ab. Eine Analyse gesamtwirtschaftlicher Diffusionsprozesse erfolgt nicht.

II. GANG DER UNTERSUCHUNG

Die vorliegende Arbeit ist in drei Teile gegliedert. Zunächst werden die grundlegenden gesamt- und einzelwirtschaftichen Zusammenhänge der Innovationstätigkeit analysiert. AnschlieBend erfolgt eine Untersuchung des Einflusses von steuern und Finanzierungsbedingungen au die Risiko- und Innovationsbereitschaft. Im dritten Teil werden dann die Möglichkeiten und Grenzen einer marktwirtschaftlichen Innovationspolitik in der Demokratie diskutiert.

Die innovationspolitischen Aktivitäten bauen auf der Hypothese auf, daß es Innovationen sind, die mittel- und langfristig wirtschaftliches Wachstum sichern. In Kapitel B wird deshalb die Innovationstätigkeit in ihren gesamtwirtschaftichen Zusammenhang gestellt. Zur oberprüfung der angeführten Hypothese werden die einzelnen Determinanten des wirtschaftswachstums unterschieden. Anschließend wird die Entwicklung dieser Bestimmungsgrößen unter Heranziehung empirischer Arbeiten analysiert.

In den Kapiteln $C$ und $D$ werden die einzelwirtschaftlichen Grundlagen der Innovationstätigkeit und der Innovationspolitik untersucht. Zunächst werden in Rapitel $C$ die Besonderheiten von Innovationsentscheidungen herausgearbeitet, indem einem einfachen neoklassischen Investitionsmodell der idealtypische Verlauf von Innovationsprozessen gegenübergestellt wird.

In Rapitel D wird der Kalkül zur Bestimmung des optimalen Innovationszeitpunkts aufgestellt. Anschließend wird der Einfluß der Wettbewerbsbedingungen und der Gewinnerwartungen auf das Innovationsverhalten analysiert. 
Im zweiten Teil der Arbeit wird die Bedeutung der steuerlichen Bedingungen und der Finanzierungsmöglichkeiten für die Risiko- und Innovationsbereitschaft untersucht. In Rapitel $E$ werden die wirkungen der Besteuerung auf die Bereitschaft zur Risikoübernahme diskutiert. Zunächst wird die Risikowirkung der Ertragsbesteuerung in einem Portefeuille-Modell über die struktur der im Portefeuille gehaltenen Titel bestimmt. Ein anschließend verwendeter neuer Ansatz setzt bei der Bestimmung der Risikowirkungen der Besteuerung direkt an der Marginalbedingung an und stellt auf die Beantwortung der Frage ab, wie die steuererhebung auf Entscheidungen zwischen zwei Alternativen wirkt, die vor steuern als gleichwertig erachtet werden und die risikolos bzw. risikobehaftet sind. Mit diesem Ansatz werden dann auch die Auswirkungen von Tarifānderungen auf die Risikobereitschaft sowie die Bedeutung von Verlustausgleichsregelungen bei schwankenden Einkommen für die Risikoübernahme analysiert. Zudem wird in diesem Abschnitt der Einfluß steuerpolitischer Aktivitäten auf das Ausmaß des steuerlichen Risikos diskutiert.

Gegenstand von Kapitel $F$ sind die für Innovationsentscheidungen besonders bedeutsamen Finanzierungsbedingungen. Zwei Fragestellungen werden hier angesprochen. Einmal wird die steuerliche Behandlung der Rapitalbildung und der alternativen Wege der Rapitalbeschaffung analysiert. Da diese Untersuchung eine eindeutige Präferenz zugunsten einer bestimmten Finanzierungsform, der Fremdfinanzierung, ergibt, wird anschlieBend erörtert, ob die kapitalsuchenden, innovationsbereiten Unternehmen diese Präferierung bei der Kapitalbeschaffung berũcksichtigen und nutzen können oder ob sie aufgrund von Restriktionen seitens der Kapitalgeber bzw. wegen der institutionellen struktur der Rapitalmärkte andere strategien verfolgen müssen.

Auf der Basis der vorangehenden Untersuchungen werden im dritten Teil der vorliegenden Arbeit die Möglichkeiten und Grenzen innovationspolitischer Aktivitäten in einer marktwirtschaftlichen Demokratie diskutiert. Zunächst werden in Kapitel $G$ die Voraussetzungen für eine zielgerichtete Innovationspolitik untersucht. Die Ausführungen konzentrieren sich 
auf die Informationsanforderungen, die eine solche Politik stellt, und auf die Bedeutung marktlicher und politisch-ökonomischer Reaktionen für die Wirksamkeit innovationspolitisch motivierter Interventionen. Innovationen sind neuartige wirtschaftiche Rombinationen von Angebot und Nachfrage. Ihre strukturen sind im vorhinein nicht bekannt. Da aber lenkende staatliche Interventionen Bezugspunkte erfordern, an denen die Maßnahmen anknüpfen, ist eine induzierende Innovationspolitik nicht möglich. Die Möglichkeiten der Innovationspolitik bestehen deshalb in einer akkommodierenden strategie, die auf den Abbau von Innovationshemmnissen ausgerichtet ist.

Rapitel $\mathrm{H}$ bietet einen Oberblick über die innovationspolitischen Aktivitäten in der Bundesrepublik Deutschland. Obwohl die Grundsätze der Politik ordnungspolitisch ausgerichtet sind, werden zahlreiche selektive Eingriffe gerechtfertigt. Die Begründungen für die innovationspolitischen Maßnahmen und die Realisierung der Fördermaßnahmen werden kritisch analysiert. Der gegenwärtig praktizierten Politik werden dann Vorschläge für eine akkommodierende Innovationspolitik gegenübergestellt, die aus dem in Rapitel $G$ vorgestellten Ronzept für eine solche strategie und den Ergebnissen dieser Arbeit entwickelt werden. 
TEIL 1: GRUNDLEGENDE GESAYT- UND EINZELHIRTSCHAFTLICHE ZUSAMTENEINGE

B. WIRTSCHAFTSWACHSTUM UND PRODURTIVITATSENTWICRLUNG

I. ROMPONENTEN DES WIRTSCHAFTSWACHSTUMS

In diesem Kapitel soll die Bedeutung der Innovationstätigkeit für die Produktivitätsentwicklung und das Wirtschaftswachstum untersucht werden. Dabei wird die Hypothese überprüft, daß es letztlich Innovationen sind, aus denen langfristig Wirtschaftswachstum resultierti).

Unter wirtschaftichem Wachstum wird hier die Zunahme des realen Sozialprodukts, also der Menge der in einer Volkswirtschaft in einem bestimmten Zeitraum erzeugten Güter und Dienstleistungen verstanden. Grundsätzlich lassen sich zwei Quellen des Wirtschaftswachstums unterscheiden ${ }^{2}$ ). Einerseits kann die Outputsteigerung durch eine Erhöhung der Inputmengen bei gegebener Produktivität erzielt werden. Andererseits kann mit gegebenem Input eine größere Ausbringungsmenge erstellt werden, wenn eine Produktivitässteigerung realisiert wird.

Das Wirtschaftswachstum aufgrund erhöhten Faktoreinsatzes kann seinerseits in zwei Rategorien unterteilt werden. Zum einen kann mit dem zusätzlichen Input die Produktion bereits im Sozialprodukt enthaltener Güter und Dienste erhöht werden. In diesem Fall handelt es sich um einen reinen Mengeneffekt. Zum zweiten kann eine innovative Komponente unterschieden werden. Zusātzliche Inputs können aber auch zur Erstellung von Gütern und Diensten verwendet werden, die bisher nicht im Sozialprodukt enthalten waren. Die Inputsteigerung dient in diesem Fall zur Produktion neuartiger Güter und Dienste. Voraussetzung dieser Art des Wirtschaftswachstums sind Produktinnovationen.

Im folgenden werden die Determinanten der Produktivitätsentwicklung im einzelnen analysiert.

1) Vgl. Bundesminister für Forschung und Technologie (1984), S. 10, 34ff

2) Vgl. Sato, R./Suzawa, G.S. (1983), S. $3 f$ 
Bei der Analyse der Determinanten der Produktivitätsentwicklung können zwei Gruppen von Faktoren unterschieden werden ${ }^{1}$ '. Die erste Gruppe von Bestimmungsgrößen führt dazu, daß bei sonst gleichen Bedingungen Produktivitätssteigerungen durch verbesserte Produktionsverfahren und durch Ausdehnung der Produktionsmengen, also durch Prozeßinnovationen und Economies of scale erzielt werden können. Diese Produktivitätssteigerungen beruhen auf veränderten Produktionsbedingungen. Die zweite Rategorie von Determinanten der Produktivitäsentwicklung ist in der für die erste Gruppe geltenden ceterisparibus-Bedingung enthalten. Sie umfaßt solche Faktoren, welche verhindern, daß bei gegebenen technischen Bedingungen und gegebenen Marktvolumina die Produktivität maximiert wird. Diese, die Realisierung einer effizienten Produktion störenden Faktoren sind sowohl in der Qualität und Mobilităt der Produktionsfaktoren Arbeit und Rapital begründet, als auch durch institutionelle und gesamtwirtschaftliche Einflüsse verursacht.

Im folgenden werden die einzelnen Bestimmungsgrößen der Produktivitätsentwicklung jeweils unter der Annahme analysiert, daß die anderen Determinanten unverändert bleiben.

\section{Prozeßinnovationen und Economies of Scale}

Die produktivitätssteigernden Wirkungen von Prozeßinnovationen und Economies of Scale lassen sich graphisch in einem einfachen Ein-Produkt-Zwei-Faktoren-Modell darstellen.

Auf den Koordinatenachsen werden die Inputmengen der Produktionsfaktoren Arbeit und Kapital abgetragen. Die Isoquanten 0 und $O^{\prime}$ sind Kurven konstanter Outputmengen bei alternativen Mengenkombinationen der beiden Produktionsfaktoren.

1) Vgl. Denison, E.F. (1979), S. 64 
Abbildung B.1: ProzeBinnovationen

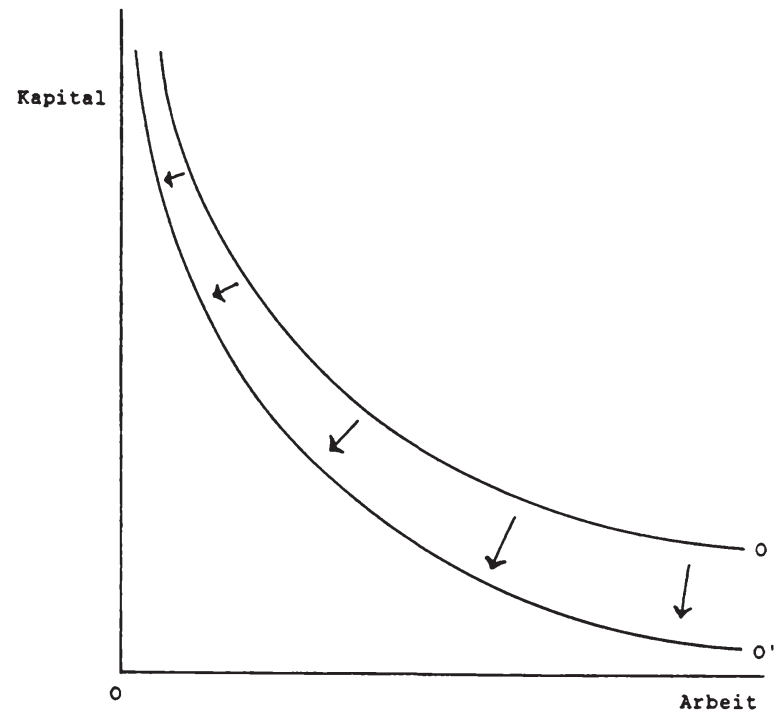

Eine Prozeßinnovation führt dazu, daß unter den neuen technischen Bedingungen die gleiche Produktionsmenge mit geringerem Faktoreinsatz als zuvor hergestellt werden kann. Graphisch läßt sich eine Prozeßinnovation als Verschiebung der Rurve konstanten Outputs zum Roordinatenursprung hin darstellen ( $O$ $\left.\Rightarrow O^{\prime}\right)$. 0 und $0^{\prime}$ repräsentieren somit das gleiche Outputniveau. Das Faktoreinsatzverhältnis bleibt dabei nur zufällig konstant. Entscheidend ist, daß die neue Isoquante im Bereich der jeweils relevanten Isokostenkurve unterhalb der ursprünglichen Isoquante liegt.

Während Produktivitätssteigerungen bei Prozeßinnovationen durch eine Anderung der technischen Bedingungen ausgelōst werden, resultieren sie im Falle von Economies of scale aus Erhöhungen des Ausbringungsniveaus .

Der Begriff der Economies of Scale (wörtlich: Ersparnisse durch Grōßenverhältnisse) beschreibt die Entwicklung der Produktionskosten bei steigerungen der Ausbringungsmenge. Je höher das Produktionsniveau, desto geringer ist der Faktoraufwand je erzeugter outputeinheit. 
Abbildung B.2: Economies of Scale

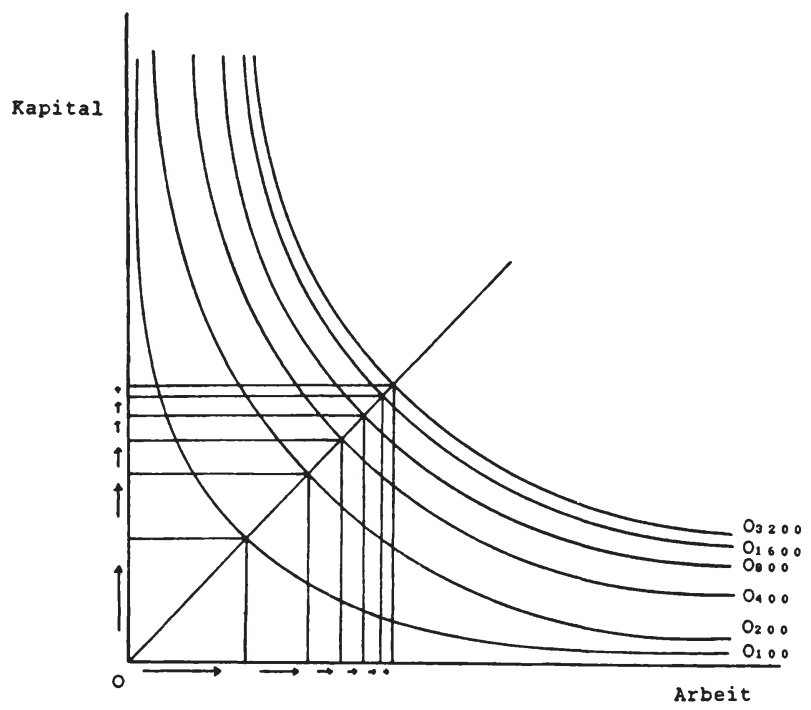

In der graphischen Darstellung bedeutet dies, daß bei schrittweiser Ausdehnung der Produktion um die gleiche Menge der Abstand zwischen den Isoquanten nach und nach abnimmt. Für die zusätzliche produktion muß also eine immer geringere Faktormenge bereitgestellt werden.

Formal läßt sich dieser Tatbestand folgendermaßen darstellen. Die Produktionsbedingungen seien beschrieben durch:

(B.1)

$\theta r \cdot 0=f(\theta \cdot I)$,

mit I: Input

o: Output

o: Parameter der Inputvariation

$r$ : Skalenfaktor

Die skalenelastizität $\varepsilon$ sei definiert als:

(B.2)

$\varepsilon=d o / 0: d I / I$.

Ist das Outputwachstum größer als die Inputsteigerung, also $\varepsilon>1$, ist auch $r>1$. 
Mit

(B.3) $\quad \mathrm{O}_{2}=\theta^{r} \cdot \mathrm{O}_{1}$

und

(B.4) $\quad I_{2}=\theta \cdot I_{1}$

sowie

(B.5) $r>1$ und $\theta>0$

läßt sich auch die Produktivitätswirkung der Economies of scale ermitteln:

$$
P_{2}=\frac{O_{2}}{I_{2}}=\frac{\theta^{r} \cdot O_{1}}{\theta \cdot I_{1}}
$$

$\Longrightarrow P_{2}>P_{1}$

Grund für Economies of Scale sind die Vorteile der Spezialisierung, die bei großen Produktionsmengen ausgenutzt werden können'). Durch die Arbeitsteilung können sich die Arbeitskräfte auf eine Aufgabe bzw. einen Produktionsvorgang konzentrieren. Zum einen gewinnen sie dadurch bezüglich dieser einen Tätigkeit größere Fertigkeiten und Erfahrung, zum anderen fällt der Zeitaufwand für Umstellungen weg. Auf der Kapitalseite sind zwei Aspekte zu unterscheiden. Zum ersten können Economies of scale dadurch entstehen, daß bereits vorhandene Kapazitäten stärker ausgelastet und deshalb die fixen Rosten auf mehr Produktionseinheiten verteilt werden. Zum zweiten sind auch auf der Kapitalstockseite spezialisierungsvorteile zu erzielen, da bestimmte Technologien erst bei großen Produktionsmengen eingesetzt werden können bzw. erst dann Anlagen speziell für einzelne Arbeitsvorgãnge bereitgestellt werden können, so daß Umrüstungskosten entfallen.

Die bisher angeführten Argumente liefern die produktionstechnischen Grundlagen für das Auftreten von Economies of Scale. Um die skalenvorteile zu realisieren, sind bei den einzelnen Produzenten Outputerhöhungen erforderlich. Diese können einerseits durch Ausdehnung der bestehenden bzw. Erschließung

1) Vgl. Wilson, J.H. (1981), S. $134 f f$ 
neuer Märkte erfolgen, oder andererseits durch eine Verringerung der Anzahl der Produzenten auf den bestehenden Märkten erreicht werden. Somit gewinnen im Bereich der Economies of Scale staatliche Eingriffe in Form von Import- und Exportregulierungen sowie Wettbewerbs- und Fusionskontrollen Einfluß auf die Produktivitätsentwicklung' '.

2. Produktivitätsverluste durch suboptimale Faktorallokation

Bei gegebener Technik und gegebenem Produktionsvolumen wird das Produktivitätsmaximum dann erreicht, wenn die angestrebte Ausbringungsmenge mit minimalem Aufwand hergestellt wird.

Abbildung B. 3: Suboptimale Faktorallokation

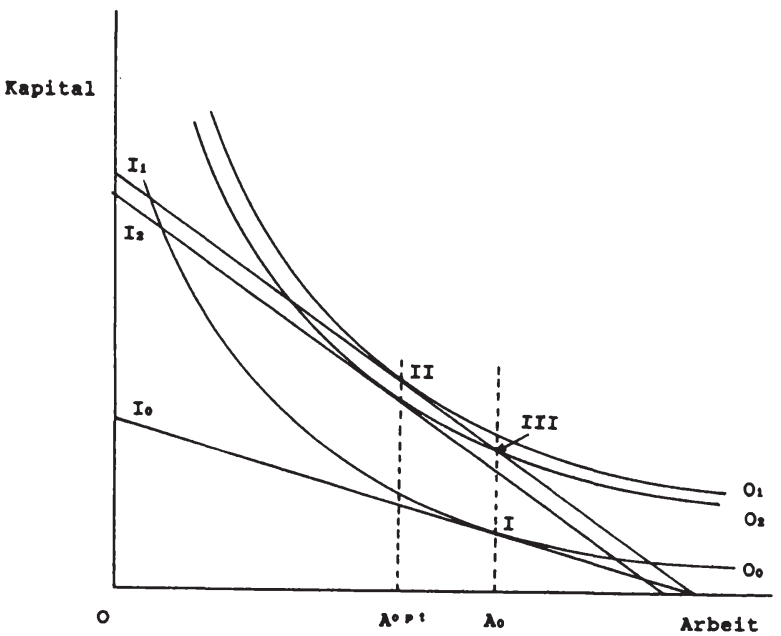

In der graphischen Darstellung wird die optimale Faktorkombination durch den Tangentialpunkt der Isoquanten und der Isokostenkurve, deren steigung das (negative) Faktorpreisverhältnis angibt, bestimmt.

1) Vgl. dazu auch Abschn. 2.2.2 
Zahlreiche Faktoren führen jedoch dazu, daß zu höheren als den minimalen Rosten produziert wird. Die Auswirkungen einer solchen Regelung auf Entscheidungen über Faktorkombinationen werden in $\mathrm{Abb}$. B.3 für eine relative Verteuerung des Faktors Arbeit dargestellt. Beim ursprünglichen Faktorpreisverhältnis repräsentiert Punkt I, der Tangentialpunkt der Isokostenkurve Io mit der Isoquante $O_{0}$, die optimale Faktorkombination. Nach einer relativen Verbilligung des Faktors Kapital (Drehung von Io nach $I_{1}$ ) würde das neue Optimum auf der Isoquante $O_{1}$ in Punkt II erreicht. Wird aber die Freisetzung von Arbeitskräften durch gesetzliche oder vertragliche Regelungen verhindert, so wird die Minimalkostenkombination verlassen und nur das Outputniveau $\mathrm{O}_{2}$ erreicht. In Punkt III ist deshalb die Produktivität suboptimal. Analoge Ronstellationen lassen sich bei entsprechenden subventionen bzw. Regulierungen für den Faktor Kapital zeigen.

Im folgenden sollen zunächst die von den Produktionsfaktoren Arbeit und Rapital ausgehenden Produktivitätsdeterminanten analysiert werden. Anschließend werden die institutionellen und gesamtwirtschaftlichen Bestimmungsgrößen der Produktivitätsentwicklung diskutiert.

\subsection{Produktivitātsdeterminanten der Inputfaktoren Kapital und Arbeit}

\subsubsection{Faktorwanderung und Faktormobilität}

Kennzeichnend für nicht-stationäre Volkswirtschaften sind Verschiebungen in der relativen Bedeutung von Branchen und Sektoren. Die Rolle einzelner Industrien ist abhängig von ihrer Produktivitätsentwicklung und von der Nachfrage nach ihren Produkten. Wandern die Produktionsfaktoren in die produktiveren Verwendungen, so erhöht sich die gesamtwirtschaftliche Produktivität'). Vielfältige Gründe verzögern jedoch diese Wanderungsbewegungen und verursachen ein Hinterherhinken

1) Vgl. Klodt, H. (1984), S. $24 f$ 
der tatsächlichen hinter der bei den gegebenen Bedingungen möglichen Produktivitãtsentwicklung. Mobilitätshemmnisse können zum Beispiel starke regionale Bindungen der Arbeitskräfte sein, vertragliche und gesetzliche Regelungen, welche die Rosten der Wanderung erhōhen (zum Beispiel der Verlust von Betriebsrenten oder die Grunderwerbsteuer), staatliche Eingriffe, die der Erhaltung veralteter strukturen dienen (zum Beispiel Subventionen der Eisen- und stahlindustrie, des Bergbaus und der Landwirtschaft oder Investitionsvergünstigungen in bestimmten Regionen bzw. für einzelne Industriezweige).

2.1.2 Faktorpreisentwicklung und Faktorsubstitutionsmöglichkeiten

Bei gegebenem Erlōs für die hergestellten Erzeugnisse hat eine Erhöhung der Faktorpreise ein Absinken der Produktivität zur Folge:

$$
\mathrm{dP} / \mathrm{dI}=-0 / I^{2}<0 .
$$

Ist ein Unternehmen ohne Einfluß auf die Faktorpreisbildung und bleiben die relativen Faktorpreise unverändert, so kann es die Produktivitätssenkung nicht kompensieren. Verschieben sich die relativen Faktorpreise, dann kann durch verstärkten Einsatz des relativ billiger gewordenen Faktors ein Teil der Produktivitätseinbuße ausgeglichen werden. Grundlage fūr eine produktivitätserhöhende substitution von Produktionsfaktoren sind zum einen eine entsprechende Flexibilität der Produktionsstruktur und zum zweiten die Fähigkeit der Unternehmung, den relativ teuereren Faktor auch tatsāchlich freisetzen zu können. Je nach struktur des produktionsprozesse kann eine Faktorsubstitution überhaupt unmöglich sein (limitationale Faktoreinsatzverhältnisse) oder die Umstellungskosten übersteigen den Preisvorteil des relativ güntigeren Faktors. Auch kann eine substitution daran scheitern, daß gesetzliche oder vertragliche Regelungen die Freisetzung von Faktoren verhindern (zum Beispiel Arbeit) oder daß keine Faktoren entsprechender Qualität zur Verfügung stehen. 


\subsubsection{Faktorqualität}

Anderungen in der Qualität der Produktionsfaktoren äußern sich in Anderungen des Outputs bei gegebenem Inputvolumen. Binzelne, die Qualität der Inputs bestimmende Aspekte sollen im folgenden getrennt für die beiden Produktionsfaktoren Arbeit und Rapital untersucht werden.

\subsubsection{Bestimmungsgrößen der Qualität der Arbeitsleistung}

Grundlegende Determinanten der Qualităt des Produktionsfaktors Arbeit sind das allgemeine Bildungs- und das berufsspezifische Ausbildungsniveau. Die Ausbildung beeinflußt entscheidend die Möglichkeiten der Arbeitsplatzwahl und die Fãhigkeiten, bestimmte Aufgaben auszuführen. Verbesserte Ausbildung ermöglicht die obernahme anspruchsvollerer Tätigkeiten und die Erledigung gegebener Aufgaben mit geringerem Aufwand. Außerdem erhöht eine breitere Allgemeinbildung die Aufgeschlossenheit und Obernahmebereitschaft gegenüber neuen Produktionsmethoden. Zudem sind sich besser ausgebildete Arbeitskräfte ihrer Fähigkeiten und Arbeitsmöglichkeiten bewußter und können deshalb leichter die Positionen erreichen, in denen ihr Grenzprodukt am größten ist' ').

Allerdings ist es zweifelhaft, ob stāndige Verlängerungen der Ausbildungszeiten die Faktorqualität auch tatsächlich erhöhen ${ }^{2}$ ). Der Bildungssektor ist hoch subventioniert und Ausbildung wird oft nur als Ronsumgut betrachtet. Zudem kann die Ausbildungsqualitāt nicht als konstant vorausgesetzt werden. Da auch der Markttest für Humankapital sehr problematisch ist, ist es durchaus denkbar, daß die Grenzerträge verlängerter Ausbildungszeiten auf null absinken oder sogar negativ werden kōnnen.

Bildung und Ausbildung haben ihrerseits Auswirkungen auf den institutionellen Rahmen der Wirtschaftstätigkeit. So ist die Formulierung und Durchsetzung von Gruppeninteressen auch von

1) Vgl. Denison, E.F. (1979), S. 42

2) Vgl. Baily, H.N. (1981), S. $10 \mathrm{f}$ 
Bildung und beruflicher Qualifikation ihrer vertreter abhängig.

Neben der Ausbildung verschafft auch Erfahrung die Fähigkeit, gegebene Aufgaben mit geringerem Arbeitseinsatz zu erledigen ${ }^{1}$. Verschiebungen in der Altersstruktur der Beschäfigten sind deshalb nicht ohne Bedeutung für die Arbeitsproduktivität. So kann zum Beispiel eine starke Erhöhung des Anteils der neu in das Erwerbsleben eintretenden Beschäftigten zu einem (vorübergehenden) Absinken der Produktivität führen.

\section{$\underline{2.1 .3 .2}$ Bestimmungsgrößen der Qualität des Sachkapitals}

Als eine erste wichtige Bestimmungsgröße der Qualitāt des Sachkapitals ist die Altersstruktur des eingesetzten Rapitalstocks zu nennen'). Mit zunehmendem Alter des eingesetzten Rapitals sinkt der Anteil der mit neuester Technik ausgestatteten Rapitalgüter. Entsprechend bleibt die tatsächliche Entwicklung der Produktivität hinter der zurück, wie sie beim Einsatz modernster Anlagen möglich wäre.

Die mit erhöhtem Alter der Kapitalgüter steigenden Ausfallund Reparaturzeiten sind ein Grund dafür, daß die effektive Einsatzzeit der Anlagen absinkt. Eine Verkürzung der Nutzungsdauer der Kapitalgüter führt jedoch nicht zwangsläufig zu positiven Produktivitätseffekten. Durchgreifende Produktivitătswirkungen können oft nur durch den Austausch von langlebigen Wirtschaftsgütern erzielt werden. Anlagen mit kurzer Nutzungsdauer enthalten hăufig nur relativ geringfügige Verbesserungen, welche die Produktivität des gesamten Rapitalstocks nur unwesentlich beeinflussen.

Für die Altersstruktur und technische zusammensetzung des Rapitalstocks ist das Investitionsvolumen die entscheidende Größe. Je höher die Ersatzinvestitionen und damit die Erneuerungsrate des Rapitalstocks und je bedeutender das Ausma $\beta$ der Erweiterungsinvestitionen, desto schneller wird der Rapitalstock mit technisch verbesserten Anlagen durchsetzt; d.h.

1) Vgl. Baily, K.N. (1981), S. $8 f$

2) Vgl. Rlodt, H. (1984), S. 62 
d.h. mit höherem Investitionsvolumen wird die Diffusion von Prozeßinnovationen beschleunigt und die Produktivität des eingesetzten Rapitals erhöht.

\subsubsection{Leistungsintensität der eingesetzten Faktoren}

2.1.4.1 Bestimmungsgrößen der Leistungsintensität des Arbeitsoinsatzes

Bei der Analyse der Leistungsintensitāt des Arbeitseinsatzes sind zwei Fragestellungen zu unterscheiden'). Zum einen ist zu untersuchen, welche Größen das Verhältnis der bezahlten Arbeitszeit zur effektiven Arbeitszeit bestimmen. Zum zweiten ist $z u$ analysieren, wodurch die Intensität der Arbeitsleistung während der zeit bestimmt wird, in der sich die Beschäftigten am Arbeitsplatz befinden.

Das Verhältnis von bezahlter zeit zu der zeit, während der die Arbeitskräte an ihrem Arbeitplatz zur Verfügung stehen, ist weitgehend durch institutionelle Bedingungen festgelegt. Zunächst führen die gesetzlichen Feiertage zu einer Diskrepanz zwischen bezahlter und verfügbarer Arbeitszeit. Ein zweiter Aspekt ist die Anzahl der (bezahlten) Urlaubstage. Sie wird gesetzlich und in Tarifverhandlungen festgelegt.

Ein dritter Punkt sind die vom Unternehmen zu bezahlenden Krankheitstage. Zwar werden die gesetzlichen und vertraglichen Regelungen zur Lohnfortzahlung ohne Mitwirkung des einzelnen Unternehmens festgelegt, der Eintritt des Rrankheitsfalles selbst ist aber in gewissem Umfang vom Unternehmen zu beeinflussen. So können zum Beispiel das Auftreten von Berufskrankheiten durch entsprechende Vorkehrungen bzw. Umgestaltungen des Arbeitsprozesses und auch die Ausnutzung von Karenztagen zum "Rrankfeiern" durch Verbesserungen der Anreizstruktur reduziert werden. Außerdem sind Fort- und Weiterbildungsmaßnahmen zu erwăhnen. Sie führen zwar zu Fehlzei-

1) Vgl. Baily, M.N. (1981), S. $14 f$ 
ten, machen aber Produktivitätsfortschritte durch verbesserte Qualifikation möglich.

Werden Nachfrageschwankungen, die zu einer Unterauslastung der Produktionsfaktoren führen, außer acht gelassen, so können zwei Faktorgruppen unterschieden werden, die die Arbeitsintensität während der am Arbeitsplatz verbrachten zeit bestimmen'). Zunächst ist auf die Motivation, d.h. die individuellen Arbeitsanreize abzustellen'). Einmal hängt sie davon $\mathrm{ab}$, ob die Fähigkeiten des Beschäftigten den Anforderungen seines Arbeitsplatzes entsprechen. Eine weitere Bestimmungsgrōße der Motivation ist die Art der Bezahlung. Ist sie leistungsabhängig, besteht ein monetärer Anreiz zu erhöhten Anstrengungen, die zu Produktivitätssteigerungen führen können. Als weitere Aspekte, welche die Leistungsbereitschaft beeinflussen, sind die mit der Arbeit verbundenen Gefahren, die Arbeitsorganisation und die Arbeitsumgebung sowie die sozialen Leistungen des Unternehmens zu nennen.

Ein in der öffentlichen Diskussion hăufig auftauchendes Argument betrifft jahrgangsspezifische (cohort-specific) Unterschiede in der Arbeitsfähigkeit und der Arbeitswilligkeit ${ }^{3}$. Aufgrund schulischer, sozialer und politischer Erfahrungen können die Arbeitsproduktivitäten einzelner Jahrgănge voneinander abweichen. Die Auswirkungen der Motivation auf die Arbeitsproduktivität werden jedoch durch die Rontroll- und Sanktionsmöglichkeiten des Arbeitgebers begrenzt. Die Beschäftigten sind um so stärker bemüht, das vom Arbeitgeber erwartete Produktionsniveau zu erreichen, je größer die Rosten des Arbeitsplatzverlustes sind und je höher die Wahrscheinlichkeit ist, daß Arbeitsleistungen, die von den Erwartungen nach unten abweichen, entdeckt und geahndet werden').

1) Vgl. Heisskopf, T.E./Bowles, S./Gordon, D.M. (1983), S. 392ff

2) Vgl. Weisskopf, T.E./Bowles, S./Gordon, D.M. (1983), S. 395

3) Vgl. zu diesem Argument das Schlagwort "people don't want to work any more", Denison, E.F. (1979), S. 134; vgl. außerdem Baily, M.N. (1981), S. $11 \mathrm{ff}$.

4) Vgl. Weisskopf, T.E./Bowles, S./Gordon, D.M. (1983), S. $393 f f$ 
2.1.4.2 Bestimmungsgrößen der Leistungsintensität des eingesetzten Sachkapitals

Abgesehen von Nachfrageschwankungen kann eine Reihe von Gründen zu einer suboptimalen Nutzung der vorhandenen Produktionsanlagen führen'). Die tatsăchliche Produktivität bleibt dann hinter der technisch möglichen zurūck. So hat zum Beispiel die Verteuerung von Rohstoffen zur Folge, daß die Nutzung von Anlagen, die diese stoffe besonders intensiv verbrauchen, reduziert wird. Wird versucht, die Rostensteigerung durch erhöhten Kapitaleinsatz aufzufangen, so bedeutet dies, daß der Faktoreinsatz bei unveråndertem output ausgedehnt wird. Die Substitution führt somit zwangsläufig zu einer Senkung der Kapitalproduktivitä. Auch haben Anderungen in den Außenhandelsbeziehungen und verschiebungen der relativen Preise Anpassungsreaktionen zur Folge, in deren Verlauf Rapitalgüter obsoleszent oder nur noch geringfügig genutzt werden. Schließlich führen restriktive Arbeitszeitregelungen und Tarifauseinandersetzungen dazu, daß die Produktionsanlagen während bestimmter zeiten nicht genutzt werden können.

2.2 Institutionelle Determinanten der Produktivitätsentwicklung

Gegenstand dieses Abschnitts sind solche staatlich gesetzten Institutionen, welche die Realisierung technisch möglicher Produktivitätsfortschritte verhindern.

\subsubsection{Requlierung von Ausnahmebereichen}

In den westlichen Volkswirtschaften werden einzelne Industriezweige dem marktlichen Proze $\beta$ entzogen und unter staatliche Rontrolle oder Regie gestellt. Dieser Art der Regulierung unterliegen insbesondere Verkehrs- und Versorgungsunternehmen (Energie- und Rommunikationsunternehmen). Die Konsti-

1) Vgl. Baily, M.N. (1981), S. $15 \mathrm{ff}$ 
tuierung von Ausnahmebereichen erfolgt mit Verweisen auf Besonderheiten in der Produktionsstruktur (Vorliegen von natürlichen Monopolen'), oder auf die herausragende politische Bedeutung (Versorgungssicherheit, Meinungsvielfalt) dieser Industrien. Auf diese Begründungen soll hier nicht eingegangen werden. Nur die Produktivitătseffekte der staatlichen Leistungen sollen diskutiert werden.

Durch Marktzutritts-, Mengen- und Preisregulierung versucht der staat, eine "angemessene" Versorgung der Bevölkerung sicherzustellen. Um diese zielsetzung zu realisieren, werden die Preis- und Mengenbeschränkungen so gestaltet, daß den regulierten Unternehmen eine "angemessene" Verzinsung ihres eingesetzten Kapitals verbleibt'). Die Bindung des Gewinns an das eingesetzte Kapital führt bei gewinnmaximierenden Unternehmen $z u$ einem überhōhten Einsatz von Kapital. Die Unternehmen versuchen, den vorgeschriebenen bzw. nachgefragten output mit einem Maximum an Kapital zu erzeugen, um dadurch ihren Gewinn zu erhöhen. Sie weichen damit aber von der Minimalkostenkombination $\left.a b^{3}\right)$. Das zusätzlich eingesetzte Rapital wird weniger produktiv eingesetzt als dies bei unverzerrten Anreizmechanismen möglich wäre. Ahnliche produktivitätsmindernde Effekte treten auf, wenn die Unternehmen in staaticher Regie stehen. In diesem Fall lautet die zielsetzung, die Versorgung der Bevölkerung kostendeckend sicherzustellen. Da im Kostenbegriff in der Regel die kalkulatorischen zinsen enthalten sind, resultieren auch hier die oben genannten verzerrungen. Ohne den vom Markt erzeugten Rostendruck kommt es zudem in anderen Bereichen zu Abweichungen von der Minimalkostenkombination. So werden Kosten verursacht, die zwar den an dem Unternehmen Beteiligten und Interessierten nutzen, fūr die Leistungserstellung selbst aber nicht erforderlich sind. Auch diese $x$-Ineffizienzen verringern die Produktivität der Unternehmen').

1) Vgl. Musgrave, R.A./Musgrave, P.B./Kullmer, L. (1987), S. $261 \mathrm{ff}$

2) Vgl. Müller, J./Vogelsang, I. (1979), S. 49

3) Vgl. Averch, H./Johnson, L.L. (1962), S. $1052 \mathrm{ff}$ sowie die Darstellung von Zajac, E.E. (1970), S. $117 f f$

4) Zum Begriff der X-Ineffizienz vgl. Leibenstein, H. (1966), S. 392ff 


\subsubsection{Wettbewerbs-, Handels- und Patentpolitik}

Die Unternehmen sind um so stärker bestrebt, ihre Rosten zu minimieren bzw. ihre Produktivität zu maximieren, je eher sie bei einer Abweichung von der Minimalkostenkombination Gefahr laufen, Marktpositionen zu verlieren und Gewinne einzubüßen. Mit der Wettbewerbs- und Handelspolitik stehen dem staat Instrumente zur Verfügung, die er zur steuerung des Produktivitåtsverhaltens der Unternehmen einsetzen kann. Die Monopolisierung von Mărkten durch Rartelle und Fusionen reduziert den Wettbewerbsdruck ebenso wie der Schutz vor ausländischer Konkurrenz durch protektionistische Maßnahmen. Ziel der Wettbewerbs- und Handelspolitik muß es deshalb sein, die Märkte offen zu halten und zu verhindern, daß die zahl der wettbewerber nicht durch andere Gründe als mangelnde Rentabilität reduziert wird.

Eine solche Politik hat allerdings zur Folge, daß jeder Marktteilnehmer neue Informationen und neue Technologien nutzen kann'). In dieser situation entfällt der Anreiz, neue Produkte und Produktionsverfahren zu entwickeln, da sich der Erfinder den Ertrag seiner Anstrengungen nicht aneignen kann. Eine Alternative besteht für ihn darin, seine Informationen geheimzuhalten. Dadurch wird aber Parallelforschung hervorgerufen und es wird verhindert, daß auf der Grundlage der bereits vorhandenen Informationen weitergeforscht werden kann. Die Patentpolitik hat deshalb eine doppelte zielsetzung. Zum einen soll, um den Anreiz zu Neuentwicklungen zu erhalten, dem Erfinder die alleinige Entscheidungsgewalt über das neue Produkt oder das neue Produktionsverfahren gesichert werden. Zum zweiten sollen, um eine effiziente Forschungsarbeit zu ermöglichen, die neuen Informationen veröfentlicht werden. Diesen Vorteilen des Patentschutzes stehen seine Rosten gegenüber, die aus der Monopolisierung') der Mărkte fūr Neuerungen resultieren. Deshalb ist die Dauer des Patentschutzes zu begrenzen. Das Patent soll theoretisch zu dem zeitpunkt

1) Zun Folgenden $\nabla g 1$. Ramien, M.I./Tauman, Y. (1984), S. 93

2) Diese Rosten umfassen nicht nur Produktivitatseinbußen sondern auch Wohlfahrtsverluste. 
auslaufen, an dem die Grenzkosten der Monopolisierung die Grenznutzen des Erfinderschutzes ausgleichen'). Da aus Gründen der Operationalität die Dauer des Patentschutzes fūr alle Neuentwicklungen pauschal festgelegt werden muß, führen die Patentgesetze zu Produktivitātseinbußen im vergleich zum theoretisch ableitbaren optimum²).

\subsubsection{Requlierung intangibler Inputfaktoren}

Zahlreiche staatliche Programme führen zu Auflagen im Unternehmenssektor. Um diesen Beschrānkungen nachzukommen, mũssen die Unternehmen Arbeit und Rapital einsetzen. Der Input wird erhöht, das Produktionsergebnis wird aber nicht gesteigert; d.h. die staatlichen Auflagen bewirken Produktivitätseinbu$\left.B^{3}{ }^{3}\right)$. Zu nennen sind in diesem zusammenhang in erster Linie die vielfäligen Auflagen zum Umweltschutz, die Maßnahmen zur Arbeitssicherheit und zum Gesundheitsschutz, aber auch zum Verbraucherschutz etc. Alle diese Programme führen dazu, daß Produktionsfaktoren zu Aufgaben eingesetzt werden mūssen, die den Output nicht erhöhen'). Auch können die Auflagen dazu führen, daß einzelne Projekte aufgrund zeitintensiver Genehmigungsverfahren verzögert oder aufgegeben werden').

\subsubsection{Besteuerung}

Besteuerungseinflüsse sind in den bisher aufgefūhrten Produktivitätsdeterminanten bereits enthalten. So beeinflußt die Besteuerung Faktorwanderungen und Faktorsubstitutionsmöglich-

1) Vgl. Nordhaus, W.D. (1969), S. $76 f f$ und Schleicher, H. (1984), S. $324 f f$

2) Vgl. Schleicher, B. (1984), S. $330 f f$ und Kaufer, E. (1985), S. 61ff

3) Vgl. Christiansen, G.B./Haveman, R.B. (1981), S. $320 f$

4) Vgl. Myers, J.G./Nakamura, L. (1980), S. 463ff. Hier werden nur die negativen Produktivităswirkungen solcher Maßnahmen dargestellt. Je nach Zielkriterium können sich diese Programme in Rosten-Nutzen-Analysen sehr wohl als sinnvoll erweisen; vgl. Denison, E.F. (1979), S. 69.

5) Vgl. Dicke, H./Hartung, H. (1986), S. $24 f f$ und Röpke, J. (1983), S. $110 \mathrm{ff}$ 
keiten, Ausmaß, struktur und qualität des Faktorangebots sowie Faktorpreise und Entscheidungen über Faktorkombinationen'). Explizit werden im Rahmen dieser Arbeit nur die Wirkungen der Besteuerung auf Innovationsentscheidungen untersucht. In diesem Abschnitt sollen nur die Bedeutung der steuerstruktur und der laufenden steuerănderungen für die Unternehmensentscheidungen betont werden'). Die steuergesetze enthalten ebenso wie die Regulierungsvorgaben zahlreiche spezialregelungen, mit denen Einzel- und Gruppeninteressen verfolgt werden. Diese führen zu Verzerrungen in der Ressourcenallokation und verhindern, daß die Produktivität beim Einsatz der Produktionsfaktoren maximiert wird ${ }^{3}$ ). Hăufige Gesetzesānderungen verursachen zudem Unsicherheit und haben eine Unterversorgung mit langfristigen und riskanten Investitionen zur Folged).

\section{$\underline{2.2 .5}$ Dienstleistungen der Unternehmen fūr den Staat}

Im Zuge der Erfüllung seiner Aufgaben verpflichtet der staat die Unternehmen zur Erbringung verschiedenster Dienstleistungen'). So sind die Unternehmen zur Abführung von Quellensteuern und Sozialversicherungsabgaben verpflichtet, obwohl sie nicht deren schuldner sind. Außerdem sind Daten $z u$ erheben, statistiken $z u$ erstellen und andere Dienstleistungen $z u$ erbringen. Diese Verpflichtung zur Erfüllung staatlich angeordneter Aufgaben führt zur Bindung von Ressourcen, die ansonsten zur Erstellung der Marktleistung der Unternehmen hätten eingesetzt werden können' ').

1) Vgl. Atkinson, A.B./Stiglitz, J.A. (1980), insbes. Rapitel 2 und 5

2) Vgl. Denison, E.F. (1979), S. $59 f$ und $131 \mathrm{ff}$ sowie Borell, R./Schemmel, L. (1987), S. $110 \mathrm{ff}$

3) Vg1. Dicke, H./Hartung, H. (1986), S. $28 f f$ und $41 f f$

4) Vgl. Dicke, H./Hartung, H. (1986), S. $38 \mathrm{ff}$

5) Vgl. Denison, E.F. (1979), S. $128 f$

6) Vgl. Dicke, H./Hartung, H. (1986), S. $19 \mathrm{ff}$ 


\subsubsection{Rent-Seeking ${ }^{1}$ )}

Mit steigendem Umfang der staatstātigkeit werden die Aktionsund Gewinnerzielungsmöglichkeiten der Unternehmen immer stärker durch staatliche Regulierungsmaßnahmen bestimmt2). Daraus resultiert für die privaten Wirtschaftssubjekte ein zunehmender Anreiz, durch den Einsatz von Ressourcen die Vergabe von Privilegien, subventionen und steuervergünstigungen bzw. die Belastung mit Abgaben zu beeinflussen. Sind die zu erwartenden Erträge aus diesen Aktivitäten größer als die aus der Markttätigkeit der Unternehmen, so verlagern sich ihre investiven Anstrengungen in den politischen Proze $\beta$. Durch diese Umschichtung der Aktivitäten, die aus einzelwirtschaftlicher sicht rational ist, werden gesamtwirtschaftliche Verluste verursacht ${ }^{3}$ ). Beim Gewinnstreben im Marktprozeß werden die Ressourcen in Verwendungen mit komparativen Vorteilen gelenkt. Daraus resultieren Wachstum und Produktivitätserhöhungen. Dagegen sind die Erträge des.Rent-Seeking das Ergebnis von Umverteilungsmaßnahmen, die zudem zu Verzerrungen der Anreizstrukturen führen. Die dafür investierten Ressourcen sind aus gesamtwirtschaftlicher sicht vergeudet. Der Wettbewerb um (Um-) Verteilungsvorteile ist ein produktivitätsminderndes Negativ-Summen-Spiel').

\subsubsection{Sonstige institutionelle Faktoren}

Die Bedeutung der Festlegung von Arbeitszeiten und Feiertagen wurde bereits bei der Erörterung der Faktornutzungsintensität angesprochen. Zudem können durch Tarifstreitigkeiten Produktivitätsverluste verursacht werden. Als weitere institutionelle Größe, die Einfluß auf die produktivitātsentwicklung hat, ist die Verteilung der staatlichen Forschungs- und Entwicklungsförderung auf zivile und militārische Projekte zu

1) Vgl. Kapitel G, Abschn. II.2.2.1.2 sowie den Oberblick bei Tollison, R.D. (1982), S. $575 f f$

2) Vgl. Olson, M. (1982), S. $143 \mathrm{ff}$

3) Vgl. Buchanan, J.M. (1980), S. 9

4) Vgl. Tullock, G. (1980), S. $269 \mathrm{ff}$ 
erwăhnen' ${ }^{\prime}$. Werden Mittel für Vorhaben aufgewendet, deren Ergebnisse keinen Einfluß auf die Produktionsprozesse haben, so geht von ihnen keine wirkung auf die Produktivitätsentwicklung aus. Dies trifft vor allem auf die militärische aber auch auf die zivile Forschung zu, die nur auf Endprodukte ausgerichtet ist und deshalb nur geringfügige Verbesserungen der Produktionsstruktur mit sich bringt.

3. Gesamtwirtschaftliche Determinanten der Produktivitätsentwicklung

\subsection{Auslastungsgrad der Produktionsfaktoren}

Die Bedeutung des Auslastungsgrades für die Produktivitãtsentwicklung läßt sich an der um die Produktionskapazität PK erweiterten Relation zwischen Output und Input veranschaulichen: ${ }^{2}$ )

$$
P=\frac{O}{I}=\frac{P R}{I} \cdot \frac{O}{P R} .
$$

Wâhrend das erste Glied des Produkts die technische Relation zwischen Rapazität und Input angibt, spiegelt das zweite Glied den Auslastungsgrad wider. Sind der Faktorbestand und damit die Produktionskapazitäten - zumindest kurzfristig nicht variabel, so führt ein Produktionsrückgang zu fallender Produktivität. Zwei gesamtwirtschaftliche Faktoren lassen sich unterscheiden, die über ihre Nachfrage- und damit Outputwirkung die Produktivitätsentwicklung beeinflussen. Zum einen bestimmt die konjunkturelle verfassung der volkswirtschaft in kurzfristiger sicht die Produktivitätsentwicklung mit $\left.^{3}\right)$. Zum zweiten sind Verschiebungen in der Nachfragestruktur langfristige Produktivitätsdeterminanten.

1) Vgl. Sato, R./Suzawa. G.S. (1983), S. $162 f$

2) Zur Darstellung vgl. Rose, R. (1964), S. 617

3) Vgl. Kendrick, J.H. (1977), S. 66 


\subsection{Inflation}

Geldwertänderungen beeinflussen die Produktivitätsentwicklung in vielfältiger Weise und auf verschiedenen Ebenen ${ }^{1}$. Zunăchst beeinträchtigen sie die Funktionsfähigkeit des Preismechanismus als Roordinations- und steuerungssystem wirtschaftlicher Aktivitäten. Die für Bntscheidungen erforderlichen Informationen über die relativen Preise erhalten die Wirtschaftssubjekte aus dem Vergleich der absoluten Preise. Indern sich diese aber ständig und insbesondere bei hohen Inflationsraten ungleichmäßig und sprunghaft, so können die Informationen über die relativen Preise nur noch schwer und unzuverlāssig gewonnen werden. Trotz höherer Kosten weichen die Wirtschaftssubjekte deshalb auf Alternativwährungen und Tauschhandel aus - "with disastrous effects on productivity"2).

Außerdem verzerrt die Inflation direkt oder indirekt Entscheidungen. Wegen erhöhter Unsicherheit über zukünftige Preise werden die Laufzeiten von Verträgen verkürzt. Entsprechend werden die Investitionsentscheidungen zugunsten kurzfristiger projekte verzerrt. Ober die steuergesetzgebung wirkt die Inflation indirekt auf wirtschaftliche Entscheidungen $^{3}$ ). Verzerrungen der Bemessungsgrundlagen und Verschiebungen der realen Tarifstruktur () führen $\mathrm{zu}$ Abweichungen von der effizienten Ressourcenallokation.

Versucht der staat, der Inflation durch restriktive Maßnahmen zu begegnen, so kommt es zu produktivitätssenkender Unterauslastung der Produktionsfaktoren bzw. im Falle von Preis- und Lohnkontrollen $\mathrm{zu}$ weiteren Erhöhungen der Transaktionskosten's).

1) Vgl. Clark, P.R. (1982), S. $149 f f$

2) Friedman, M. (1977), S. 467

3) Vol. die Beitrăge von Brinner, R.E., Davidson, S. und Veil, R.L., sowie Tideman, T.N. und Tucker, D.P. in: Aaron, H.J. (1976)

4) Vgl. Herb, F. (1986)

5) Vgl. Denison, E.F. (1979), S. 18 
Im folgenden Abschnitt werden die Determinanten der Produktivitätsentwicklung auf ihre quantitative Bedeutung hin untersucht. Dazu wird ein Oberblick über verschiedene empirische Arbeiten zur Analyse der Produktivitätsentwicklung gegeben.

III. ERGEBNISSE EMPIRISCHER FORSCHUNG ZUR PRODURTIVITATSENTWICRLUNG

Ziel der Untersuchung ist die Rlärung der Bedeutung von Produkt- und Prozeßinnovationen für Wirtschaftswachstum und Produktivitätsentwicklung. Auf methodische Unterschiede und Meßprobleme bei den verschiedenen Arbeiten wird nur am Rande eingegangen.

Da für die Bundesrepublik Deutschland nur wenige Untersuchungen zur Produktivitätsentwicklung und ihren Bestimmungsgrößen vorliegen, stützen sich die folgenden Ausführungen weitgehend auf in den Vereinigten staaten von Amerika gemachte Arbeiten.

Tabelle B.1: Entwicklung des Bruttosozialprodukts

in der Bundesrepublik Deutschland und den USA

- Durchschnittliche jährliche Wachstumsraten in Prozent -

\begin{tabular}{|l|r|r|r|r|r|r|r|}
\hline Zeitraul & 1950-1955 & $1955-1960$ & $1960-1965$ & $1965-1969$ & $1969-1973$ & $1973-1979$ & $1979-1984$ \\
\hline BR Deutschland1 & $9,1^{21}$ & 6,821 & 1,9 & 1,0 & 1,1 & 2,1 & 0,9 \\
\hline OSA') & 1,2 & 2,4 & 1,9 & 1,0 & 3,6 & 2,8 & 2,1 \\
\hline
\end{tabular}

1) Bruttosozialprodukt in Preisen von 1976

2) Ohne Saarland und Berlin (West)

3) Bruttosozialprodukt in Preisen von 1972

Quellen: Statistisches Bundesamt (1985), S. 41;

U.S. Bureau of Census, verschiedene Auflagen 
Tabelle B.1 zeigt die Entwicklung der Wachstumsraten des Bruttosozialprodukts in der Bundesrepublik Deutschland und den USA von 1950-1984. Im Untersuchungszeitraum ist ein drastischer Rückgang der Wachstumsraten bis hin zu einer Schrumpfung des realen sozialprodukts festzustellen. $\mathrm{Zu} \mathrm{Be-}$ ginn der achtziger Jahre läßt sich eine leichte Erholung feststellen.

Tabelle B.2: Produktivitätsentwicklung in der Bundesrepublik Deutschland und den USA

a) Entwicklung der Arbeitsproduktivität

- Durchschnittliche jährliche Produktivitātssteigerung in Prozent -

\begin{tabular}{|c|c|c|c|c|c|c|c|}
\hline Zeitraur & $1950-1955$ & $1955-1960$ & $1960-1965$ & $1965-1969$ & $1969-1973$ & $1973-1979$ & $1975-1982$ \\
\hline BR Deutschland1) & $6,62)$ & 4,821 & 4,4 & 4,5 & 3,6 & 3,0 & 2,5 \\
\hline Zeitraun & $1948-1953$ & $1953-1964$ & $1961-1969$ & $1969-1973$ & $1973-1976$ & $1976-1979$ & $1979-1981$ \\
\hline OSA'I & 2,6 & 2,4 & 1,5 & 1,5 & $-0,2$ & 1,541 & $0,(1)$ \\
\hline
\end{tabular}

1) Bruttoinlandsprodukt in Preisen von 1976 je Erwerbstätigem

2) In Preisen von 1962, ohne Saarland und Berlin (West)

3) Volkseinkommen je Beschäftigtem, in Preisen von 1972

4) Volkseinkommen je Beschäftigtem, deflationiert mit dem Preisindex des Bruttosozialprodukts

Quellen: Klodt, H. (1984), S. 5; Denison, E.F. (1979), S. 27 ; U.S. Bureau of Census, verschiedene Auflagen 
Eine ähnliche Entwickinng ist auch bei den Produktivitätssteigerungsraten zu beobachten. Ein Veigleich der Entwicklung der Arbeitsproduktivität in beiden Ländern (T̃belle B.2a)) zeigt, daß sich der Rückgang des Produktivitātszuwachses in der Bundesrepublik Deutschland zwar in etwa dem gleichen relativen Ausmaß wie in den USA, jedoch von einem deutlich höheren Niveau aus vollzieht.

Tabelle B.2: Produktivitätsentwicklung in der Bundesrepublik Deutschland und den USA

b) Entwicklung der totalen Faktorproduktivität

- Durchschnittliche jährliche Produktivitätssteigerung in Prozent -

\begin{tabular}{|l|r|r|r|r|r|}
\hline Zeitrauı & $1948-1953$ & $1953-1964$ & $1964-1969$ & $1969-1973$ & $1973-1976$ \\
\hline USA & 2,46 & 2,32 & 1,77 & 1,64 & 0,04 \\
\hline
\end{tabular}

1) Output: Volkseinkommen in Preisen von 1972

Input : Arbeit, Kapital (Wirtschaftsgebäude, Anlagen, Lagerbestände), Boden

Quelle: Denison, E.F. (1979), S. 104. Die Rlassifikation der Wachstumsdeterminanten wurde gegenüber der Aufteilung von Denison in der Weise geändert, daß Struktur- und Qualitätsänderungen beim Faktor Arbeit als Determinanten der Produktivität und nicht als Inputfaktoren gewertet werden.

In Tabelle B.2b) wird die Entwicklung der gesamten Faktorproduktivität in den USA dargestellt'). Sie ist definiert als das Verhāltnis des Outputs zu der summe der Inputfaktoren Arbeit, Kapital und Boden. Auch hier ist eine ausgeprägte Abnahme des Produktivitätszuwachses festzustellen.

1) Entsprechende Angaben liegen für die Bundesrepublik Deutschland nicht vor. 
Dieser Verlauf der Produktivitätsentwicklung ergibt sich unabhängig davon, welche Input- und Outputgrößen als Berechnungsgrundlagen gewăhlt werden'). Erste Untersuchungen für die USA deuten darauf hin, daß sich auch zu Beginn der achtziger Jahre die Produktivitätssteigerungen auf niedrigem Niveau bewegen ${ }^{2}$ ), jedoch mit ansteigender Tendenz ${ }^{3}$ ).

Tabelle B.3: Wachstum des Volkseinkommens und seine

Zusammensetzung in den USA 1948-1976

- Durchschnittliche jährliche Wachstumsrate des Volkseinkommens in Prozent -

- Beitrag der Wachstumskomponenten in Prozentpunkten -

\begin{tabular}{|c|c|c|c|c|c|c|}
\hline Zeitraul & $1948-1973$ & $1948-1953$ & $1953-1964$ & $1964-1969$ & $1969-1973$ & $1973-1976$ \\
\hline Volkseinkonen & 3,65 & 4,64 & 3,12 & 4,45 & 2,91 & 0,58 \\
\hline Brhöhung des Inputs & 1,53 & 2,18 & 0,80 & 2,68 & 1,27 & 0,54 \\
\hline - davon: Arbeit & 0,82 & 1,37 & 0,16 & 1,88 & 0,63 & 0,08 \\
\hline Rapital & 0,71 & 0,81 & 0,64 & 0,80 & 0,64 & 0,46 \\
\hline $\begin{array}{l}\text { Steigerung der } \\
\text { totalen Paktor- } \\
\text { produktivitat }\end{array}$ & 2,12 & 2,46 & 2,32 & 1,77 & 1,64 & 0,04 \\
\hline
\end{tabular}

Quelle: Denison, E.F. (1979), S. 104; zu den Anderungen gegenüber den Angaben von Denison vgl. Anmerkung zu Tab. B.2.b.

1) Vgl. auch die Ansătze bei Clark, P.R. (1978); Norsworthy, J.R./Harper, M.J./ Kunze, K. (1979); Baily, M.N. (1981) und (1982), sowie Weisskopf, T.E./Bowles, S./Gordon, D.M. (1983)

2) Vgl. Clark, P.K. (1984), S. $133 f f$

3) Vgl. Baily, M.N. (1984), S. $234 f$ 

hungen am Wachstum des Sozialprodukts

Tabelle B.3 enthält eine Aufgliederung des Sozialproduktswachstums. Die steigerungsraten der Produktivität sind in Prozent angegeben. Der Beitrag der Inputerhöhungen wird in Prozentpunkten angeführt. In dieser Darstellung sind die Folgen der Abnahme des Produktivitātszuwachses deutlich zu erkennen. Tragen die steigerungen der Faktorproduktivität 1948-1973 noch mit 58 Prozent zum Sozialproduktswachstum bei, so geht ihr Anteil im Zeitraum von 1973-1976 auf 7 Prozent zurūck.

3. Romponenten des Wirtschaftswachstums aufgrund erhöhten Faktoreinsatzes

wie oben ausgeführt'), kann zusätzlicher Faktoreinsatz dazu verwendet werden, die Produktion bereits am Markt eingeführter Güter auszuweiten oder um neue Produkte herzustellen. Untersuchungen über die Zusammensetzung der mit zusätzlichem Faktoreinsatz erstellten Produktion liegen nicht vor. Anhaltspunkte für die Bedeutung der Produktinnovationen können aber aus Analysen der struktur von Unternehmensumsätzen bzw. der struktur des Umsatzwachstums gewonnen werden. Eine entsprechende Untersuchung ergibt, daß 50 prozent und mehr des Umsatzes mit Produkten gemacht werden, die in einem zeitraum von zehn Jahren vor dem Untersuchungszeitpunkt entwickelt oder eingeführt worden sind2). Zum kurzfristigen Unternehmenswachstum tragen nach dieser Analyse die neuentwickelten Produkte zu 46 Prozent bis 100 Prozent bei ${ }^{3}$ ). Im Jahre 1985 machten die Produkte, die sich in der Markteinführungs- und Wachstumsphase befinden, 38 Prozent des Umsatzes aus ${ }^{4}$.

Bei der Interpretation dieser Zahlen ist mit Blick auf ihre Bedeutung für das Wachstum des Sozialprodukts zu beachten,

1) Vgl. Abschn. B.I

2) Vgl. Booz/Allen/Bamilton (1964), S. 2

3) Vgl. Booz/Allen/Hamilton (1964), S. 6

4) Vgl. Schmalholz, H./Scholz, L. (1987), S. 21 
daß bei der Aufgliederung des Sozialprodukts nur solche Neuentwicklungen als Produktinnovationen erfaßt werden, die von Endverbrauchern konsumiert werden. Alle Neuentwicklungen, die von Produzenten erworben werden, gehen als Vorleistungen in den Produktionsproze $\beta$ ein und sind deshalb Prozeßinnovationen. Dennoch kann davon ausgegangen werden, daß Produktinnovationen einen entscheidenden Anteil an dem durch erhöhten Faktoreinsatz getragenen Wachstum des Sozialprodukts haben.

\section{4. Komponenten der Produktivitätsentwicklung}

Die quantitative Bedeutung der einzelnen Determinanten der Produktivitätsentwicklung kann anhand der umfassenden Arbeit von E.F. Denison: Accounting for slower Economic Growth 1 ) dargestellt werden. Liegen zu einzelnen Bestimmungsgrößen der Produktivitätsentwicklung weitere Untersuchungen vor, werden diese ergänzend angeführt.

Tabelle B.4 enthält die von Denison herausgearbeiteten Bestimmungsgrōßen des Sozialproduktswachstums, die hier als Produktivitātsdeterminanten klassifiziert werden ${ }^{2}$ ).

Die Arbeitsqualităt ist mit einem Beitrag von rund einem Viertel zum gesamten Produktivitätswachstum über den Zeitraum 1948-1973 die bedeutendste der einzeln berechneten Produktivitätsdeterminanten. In den Jahren 1973-1976 übersteigt die Qualitätsverbesserung des Faktors Arbeit den gesamten Produktivitäsfortschritt bei weitem. Da zudem der Beitrag der Arbeitsqualität zum Produktivitätswachstum im betrachteten Zeitraum absolut konstant bleibt, liefert er keine Erklärung

1) Denison, E.F. (1979); vgl. auch die Arbeit von Kendrick, J.W. (1980), der Denisons Methode des Growth Accounting zugrunde liegt. Grundlage dieser Kethode sind Zeitreihen über die Entwicklung der einzelnen Outputdeterminanten. Ihren Verănderungen werden die Anderungen des Outputs gegenübergestellt; vgl. zur Vorgehensweise Denison, E.F. (1974).

2) Abweichungen $\mathrm{zu}$ der Aufteilung von Denison bestehen vor allem beim Faktor Arbeit. Denison weist struktur- und Qualitătsănderungen als inputsteigernde Faktoren aus, wăhrend sie hier als Produktivitătsdeterminanten klassifiziert werden; wie hier auch Rendrick, J.H. (1980), S. $3 f$. 
Tabelle B.4: Bestimmungsgrößen der Produktivitätsentwicklung in den USA 1948-1976

- Durchschnittliche jährliche steigerungsraten des Volkseinkommens und der Faktorproduktivität in Prozent -

- Beitrag der Produktivitātsdeterminanten in Prozentpunkten -

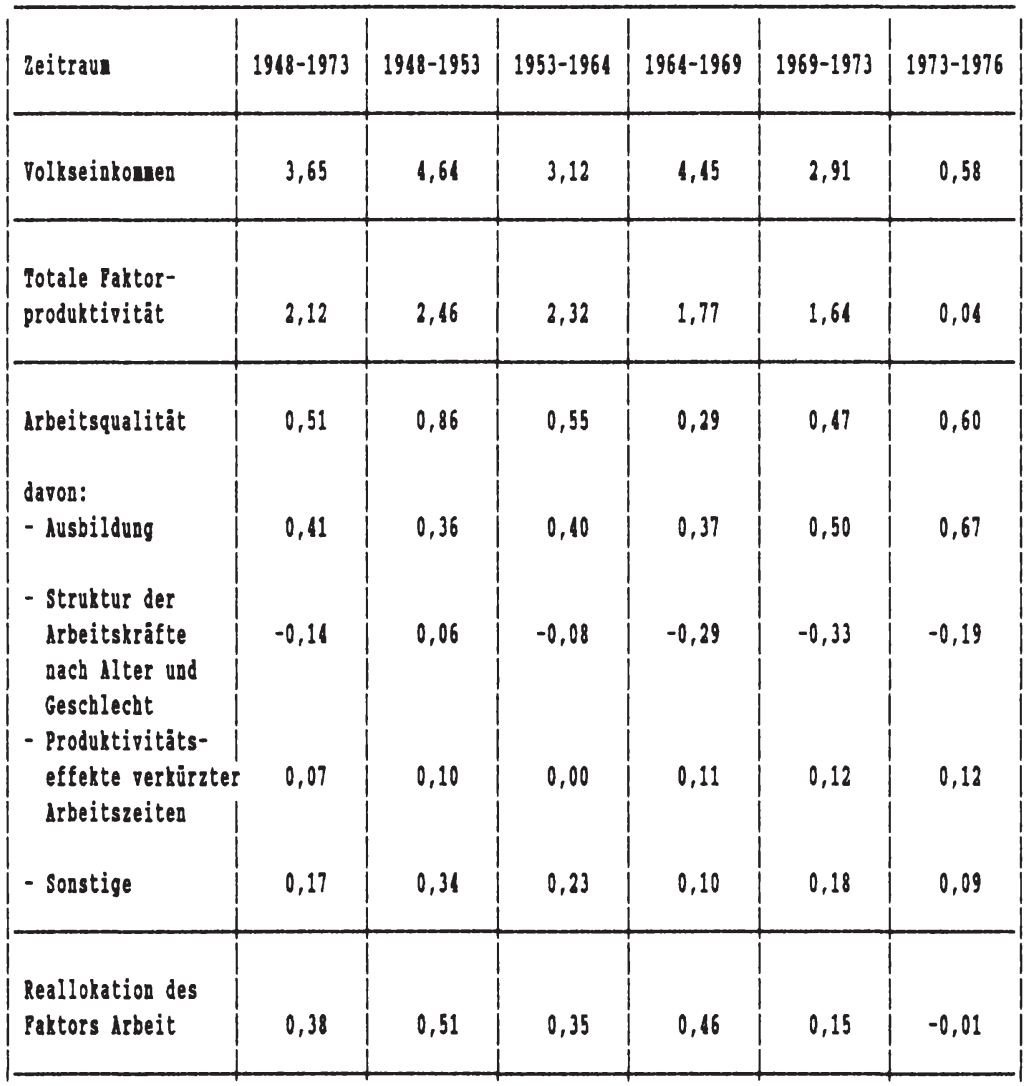


Fortsetzung Tabelle B.4

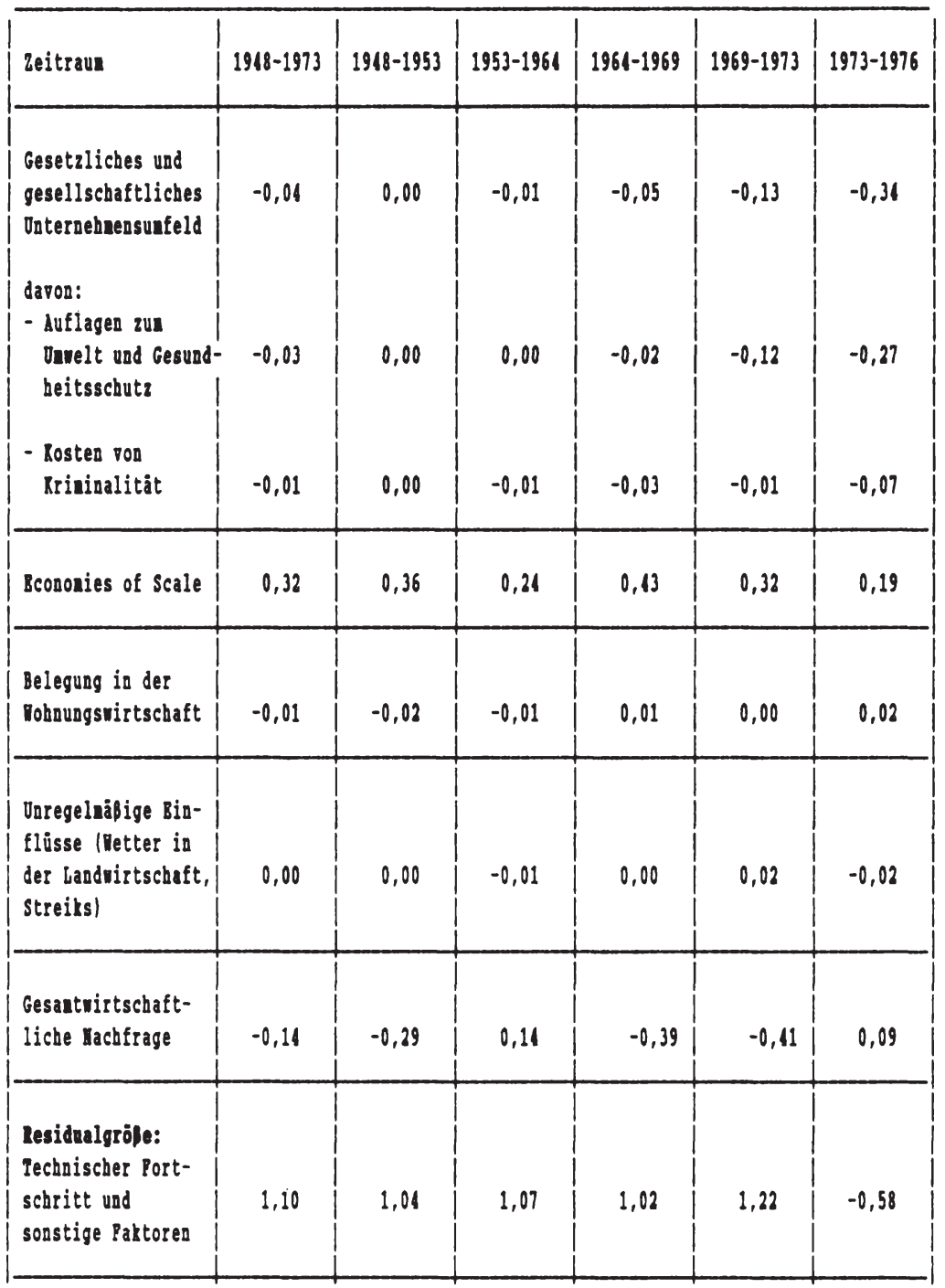

Quelle: Denison, E.F. (1979), S. 104; zu den Anderungen gegenüber den Angaben von Denison vgl. Anmerkung zu Tab. B.2.b. 
für die Produktivitātsschwäche und es muß nach Faktoren gesucht werden, welche die Produktivitātsentwicklung negativ beeinflussen. Die mit Abstand wichtigste Romponente der Qualitätssteigerung ist die Verbesserung des Ausbildungsniveaus; sie trägt über 80 prozent zur Erhöhung der Faktorqualität bei. Ein insgesamt negativer Effekt geht von Anderungen in der zusammensetzung der Beschäftigten aus. Er ist die Folge des Eintretens vieler junger und unerfahrener Arbeitskräte sowie einer Erhöhung des Anteils der Frauen an den Beschäftigten. Ein positiver Produktivitătsbeitrag wird durch die Verkürzung der Wochenarbeitszeit erreicht. Durch die Verminderung der zu leistenden Arbeitsstunden wird der Output pro zeiteinheit erhōht. Die Rubrik "Sonstige" enthält Produktivitātseffekte, die bei Arbeitskräten auftreten, welche keine Marktleistungen erstellen. Es sind vor allem Produktivitätssteigerungen aufgrund verbesserter Ausbildung von Beschäftigten im offentlichen Dienst und bei Privathaushalten. Auch sind Produktivitäseffekte aufgrund von Umschichtungen innerhalb dieser Bereiche sowie zwischen ihnen und der Privatwirtschaft enthalten').

Die Untersuchungsergebnisse von Denison zur produktivitässenkenden Anderung der Arbeitskräftestruktur werden in Arbeiten von Baily, Clark, Darby und Norsworthy/Harper/Runze bestätigt'). Die Untersuchungen zu den Produktivitätseffekten von Verānderungen der Arbeitsqualität basieren auf der Annahme, daß die Produktivitätsunterschiede von Arbeitskräften in den relativen Löhnen widergespiegelt werden, die Beschäftigten also nach ihrem Grenzwertprodukt bezahlt werden. Diese Unterstellung ist nicht unproblematisch. Zahlreiche institutionelle und gesellschaftliche Faktoren führen dazu, daß gleiche Arbeiten ungleich entlohnt werden. Wenn zum Beispiel Frauen und Farbige geringe Löhne crhalten, dann bedeutet dies nicht unbedingt, daß sie weniger produktiv sind ${ }^{3}$ ). Ihre Produktivität kann allerdings deshalb niedriger sein, weil sie

1) Vg1. Denison, E.F. (1979), S. $106 f$ und FN 4

2) Vgl. Baily, M.N. (1981), S. 8ff, Clark, P.R. (1978), S. 968ff, Darby, M.R. (1984), S. $302 f f$ und Norsworthy, J.R./Harper, M.J./Runze, R. (1979), S. $405 f f$

3) Vgl. Baily, M.N. (1981), S. $8 \mathrm{ff}$ 
bei steigendem Arbeitsangebot in weniger produktive Beschäftigungen abgedrăngt werden. Auch ist es fraglich, ob zusätzliche Ausbildung die Produktivität im Ausmaß der Lohnsteigerung erhōht. Es ist somit nicht auszuschließen, daß die wirkungen zusātzlicher Ausbildung bei Denison zu hoch ausgewiesen sind').

Zwei weitere Determinanten der Produktivitătsentwicklung, die sich auf den Produktionsfaktor Arbeit beziehen, werden von Weisskopf/Bowles/Gordon und Medoff untersucht' ${ }^{2}$. Weisskopf/ Bowles/Gordon beziehen in ihre Analyse der Produktivitätsentwicklung auch die motivationsabhängige Leistungsintensität der Arbeitskräfte ein. In ihrem Modell trägt sie entscheidend zur Erklärung der Produktivitătsschwäche bei $\left.{ }^{3}\right)$. In den beiden untersuchten zeitrăumen wird der Rückgang des Produktivitätszuwachses zu rund 80 bzw. 30 Prozent erklärt. Allerdings ist in ihrer Untersuchung die Wahl der Indikatoren der Arbeitsintensität sehr problematisch, da eine Rorrelation zwischen Indikatoren und Produktivitätsentwicklung nicht ausgeschlossen werden kann'). Medoff stellt in seiner Untersuchung fest, daß die Arbeitgeber seit 1973 trotz steigender Arbeitslosigkeit deutlich größere Anstrengungen unternehmen, Arbeitskräfte anzuwerben, die ihre Anforderungen erfüllen. Das demnach bestehende Ungleichgewicht zwischen dem Arbeitsangebot und der struktur der Arbeitsnachfrage erhöht die Aufwendungen für den Inputfaktor Arbeit und reduziert seine Produktivität's'.

Aus der Reallokation des Faktors Arbeit resultiert ungefähr ein Fünftel des Produktivitäsfortschritts im zeitraum von 1948 bis 1973. Seit dem Ende der sechziger Jahre ist jedoch ein starker Rückgang des Beitrags der Reallokationen zur Produktivitätssteigerung festzustellen. Im Zeitraum von 1973 bis

1) Vgl. Baily, M.N. (1981), S. $10 f$

2) Weisskopf, T.E./Bowles, S./Gordon, D.M. (1983), S. 381ff; Medoff, J.L. (1983), S. 87ff

3) Weisskopf, T.E./Bowles, S./Gordon, D.M. (1983), S. $421 f$

4) Vgl. Kommentare und Diskussion zu der Arbeit von Weisskopf, T.E./Bowles, S./Gordon, D.M., in: Brookings Papers on Economic Activity $2: 1983$, S. $442-450$

5) Medoff, J.L. (1983), S. $115 \mathrm{ff}$ 
1976 wirkten die Faktorwanderungen produktivitätsmindernd. Die Produktivitätssteigerungen aus der Reallokation des Faktors Arbeit ergeben sich dadurch, daß Arbeitskräfte in eine Beschäftigung wandern, in der sie ein höheres Grenzprodukt erzielen als zuvor. Denison erfaßt in seiner Arbeit zwei Wanderungsbewegungen'1). Zwei Drittel trägt der Abzug von Beschäftigten aus der Landwirtschaft in den sekundären oder tertiāren sektor zum Produktivitätsfortschritt bei. Das dritte Drittel steuert die Wanderung von selbständigen und mithelfenden Familienangehōrigen in eine abhängige Beschäftigung in der privaten Wirtschaft bei. Die Möglichkeiten, durch eine Behebung von Fehlallokationen des Faktors Arbeit Produktivitätsfortschritte zu erzielen, lassen jedoch seit dem Ende der sechziger Jahre stark nach und im Zeitraum von 1973 bis 1976 ist die Reallokation des Faktors Arbeit aus Verwendungen in der Landwirtschaft und als Selbständige keine Quelle des Produktivitätsfortschritts mehr.

Kendrick ${ }^{2}$ ) bezieht in seine Untersuchung auch die Produktivitätseffekte der Reallokation des Faktors Rapital ein. Betrug der Produktivitātsbeitrag der Reallokation von Kapital im Zeitraum von 1948 bis 1973 noch über 0,4 Prozentpunkte, so ging er im Zeitraum von 1973 bis 1978 auf 0,2 Prozentpunkte zurück.

Klodt ${ }^{3}$, vergleicht für die Bundesrepublik Deutschland die partiellen Produktivitätseffekte des strukturwandels in den Zeiträumen von 1960 bis 1970 und von 1970 bis 1980 . Es zeigen sich die gleichen Produktivitätsdifferenzen zwischen dem Agrarbereich und der übrigen Wirtschaft wie in den USA. Der strukturwandel zugunsten des tertiären und zu Lasten des primären Sektors bei relativer Ronstanz des sekundären sektors hat eine positive, jedoch abnehmende steigerungsrate der Arbeitsproduktivitāt zur Folge. Dagegen fält die steigerungsrate der Rapitalproduktivität stark. Wăhrend sie sich von 1960 bis 1970 infolge des strukturwandels um 1,8 prozent erhöht, fällt sie von 1970 bis 1980 um 0,6 prozent.

1) Vgl. Denison, E.F. (1979), S. $64 \mathrm{ff}$

2) Kendrick, J.W. (1980), S. 3 und $13 f$

3) Klodt, H. (1984), S. $24 f f$ 
Einen stark wachsenden Einfluß auf die Produktivitätsentwicklung gewinnen Faktoren aus dem Umfeld der Unternehmen. Staatliche Auflagen engen die Aktionsfreiheit der Unternehmen ein und binden Mittel, die ansonsten zur Outputerstellung hätten eingesetzt werden können. Das Ausmaß derartiger Regulierungen nimmt stark zu und entsprechend steigt ihr negativer Einfluß auf die Produktivitätsentwicklung. Die von Denison erfaßten Regulierungen im Bereich des Umweltschutzes, der Arbeitssicherheit und des Gesundheitsschutzes erklären rund zwölf Prozent des Produktivitātsrückgangs von 1969-1973 zu 1973-1976. Zwei Drittel davon sind durch Umweltschutzmaßnahmen verursacht.

In verschiedenen anderen Untersuchungen wurden produktivitätsmindernde Effekte der Regulierung in etwa gleicher GröBenordnung ermittelt').

Die zweite hier erfaßte Komponente von Faktoren aus dem Umfeld der Unternehmen, die Ressourcen in nicht produktiver Verwendung binden, sind die Folgen der Kriminalitāt. Die Aufwendungen der Unternehmen zum Schutz gegen Verbrechen und zur Beseitigung ihrer Folgen haben insbesondere im letzten hier betrachteten zeitraum einen deutlich produktivitätsmindernden Effekt.

Anderungen in der Belegungsrate der Wohnungen haben ebenso wie klimatischen Einflūsse und streiks nur geringfügige bzw. keine Produktivitātswirkungen und können den Produktivitātsrückgang nicht erklären. Sowohl die Belegung des Wohnungsbestandes als auch Tarifauseinandersetzungen haben sich im Betrachtungszeitraum nicht so verändert, als daß sie einen spürbaren Einfluß auf die Produktivitätsentwicklung gehabt hätten.

Bconomies of Scale tragen nach den Berechnungen Denisons von 1948 bis $1973 \mathrm{ca}$. 15 Prozent zum Produktivitätsfortschritt bei. Im Zeitraum von 1973 bis 1976 geht ihr absoluter prozentualer zuwachs deutlich zurück, ihr Produktivitätsbeitrag ist

1) Vgl. den Oberblick bei Christiansen, G.B./Havemann, R.H. (1981), S. 320 ff und Haveman, R./Norsworthy, J.R. (1987) sowie die Arbeit von Link, A.N. (1982), S. $548 f f$. 
aber immer noch größer als der Fortschritt der gesamten Faktorproduktivitãt.

Inderungen der gesamtwirtschaftlichen Nachfrage beeinflussen die Produktivitätsentwicklung über ihre Effekte auf die Auslastung der gegebenen Produktionsmöglichkeiten. Der negative Beitrag der gesamtwirtschaftichen Nachfrage auf die Produktivitätsentwicklung über den zeitraum von 1948 bis 1973 zeigt, daß das Produktionspotential 1948 stärker als 1973 ausgelastet war. Dagegen ist die Auslastung von 1973 bis 1976 leicht angestiegen. Der geringe Produktivitäsanstieg von 1973 bis 1976 wird also in diesem Ansatz nicht durch eine geringere Nachfrageintensitãt erklärt.

Denisons Berechnungen basieren auf dem langfristigen Trend der Auslastung des Produktionspotentials. Dieser ist seit 1948 fallend. Trotz der stärkeren Nachfrage von 1976 im Vergleich zu 1973 ist absolut gesehen die Rapazitätsauslastung in 1976 geringer als im Trend der Vorjahre'). Wird nun der konjunkturelle Einfluß auf die Produktivitätsentwicklung nicht aufgrund des Trends sondern am absoluten Auslastungsgrad gemessen, so trägt die konjunkturelle Entwicklung deutlich zum Produktivitātsrūckgang bei. Baily führt beispielsweise über zehn Prozent des Produktivitätsrückgangs nach 1973 auf eine abgeschwächte Nachfrage zurück²).

Der Beitrag des technischen Fortschritts zum Produktivitäswachstum wird bei Denison als Restgröße ermittelt. Diese blieb von 1948 bis 1973 nahezu konstant und war mit einem Beitrag von rund 1,1 Prozentpunkten die bedeutendste Quelle von Produktivitätssteigerungen. Nach 1973 nahm diese Romponente des Produktivitatsfortschritts jedoch drastisch ab und wurde negativ. Da es jedoch keine Anzeichen dafür gibt, daß der Beitrag der Forschungs- und Entwicklungsaktivitäten zum Produktivitäswachstum abgenommen hat ${ }^{3}$ ), ist zu untersuchen, ob seit 1973 Entwicklungen eingetreten sind, die in der Residualgröße enthalten sind und einen Teil der Produktivitätsschwäche erklären.

1) Vgl. Denison, E.F. (1979), S. 65

2) Baily, M.N. (1982), S. $428 \mathrm{ff}$

3) Vgl. Griliches, Z. (1986), S. $144 f f$ 
Die oben angesprochene Verwendung einer alternativen Meßmethode zur Bestimmung der konjunkturellen Binflūsse auf die Produktivitätsentwicklung hat zwar einen geringeren Rückgang der Residualgröße zur Folge. Der sprunghafte Rückgang des Produktivitätszuwachses kann jedoch nicht erklärt werden, da auch in den Vorperioden Anpassungen vorgenommen werden mü $\beta-$ ten.

Im folgenden werden deshalb verschiedene, bislang nicht berücksichtigte Entwicklungen auf ihre Produktivitätswirkungen hin untersucht.

In der bisherigen Analyse sind die Produktivitätseffekte der Wettbewerbs- und Handelspolitik nicht enthalten. Empirische Untersuchungen', zeigen, daß Industriezweige mit hōherer Wettbewerbsintensität günstigere Produktivitätsentwicklungen haben als solche mit geringerem Ronkurrenzdruck.

Werden die Marktverhältnisse in der gesamten Wirtschaft betrachtet, sind allerdings nur geringfügige Anderungen der Marktstruktur festzustellen'2). Mit abnehmendem Ronkurrenzdruck kann also der sprung in der Produktivitätsentwicklung nicht erklärt werden.

Die Belastung der Unternehmen durch Dienstleistungen, die sie für den staat zu erbringen haben, blieb bisher ebenfalls unberücksichtigt.

Nach einer Untersuchung der Commission on Federal Paperwork ${ }^{3}$ ) mußten die Unternehmen (ohne Wohnungswirtschaft) im Jahr 1976 2,4 bis 3,1 Prozent ihres Einkommens für Dienstleistungen an den Bundesstaat und etwa 3,0 bis 4,6 Prozent für Leistungen an alle staatlichen Ebenen aufwenden. Für ein besonders starkes Ansteigen dieser Belastungen nach 1973 gibt es keine Anzeichen, zumal die 1973 noch geltenden Lohn- und Preiskontrollen in der $\mathrm{Zwischenzeit} \mathrm{aufgehoben} \mathrm{wurden.}$

1) Vgl. die Arbeiten von Baily, M.N./Chakrabarti, A.K. (1985), S. 609ff mit einem Vergleich von Industriezweigen und Wagner, $\mathbb{R}$. (1980), $S$. $17 f f$ mit einem Lăndervergleich.

2) Vgl. White, L.J. (1981), S. $223 \mathrm{ff}$

3) Zitiert in Denison, E.F. (1979), S. $128 \mathrm{f}$ 
Anders verläuft die Entwicklung dieser Belastungen in der Bundesrepublik Deutschland ${ }^{1}$ ). Von 1957 bis 1983 sind die Rosten der unentgeltlichen Dienstleistungen der Unternehmen für den staat von einem Anteil von 0,5 Prozent am Bruttosozialprodukt über 1,1 Prozent im Jahr 1971 auf 3,7 Prozent angestiegen. Zum Produktivitātseffekt dieses Anstiegs liegen keine Untersuchungen vor, doch dürfte er zum Produktivitätsrückgang in der Bundesrepublik Deutschland fühlbar beigetragen haben.

Zeitgleich mit dem drastischen Absinken des Produktivitātszuwachses sahen sich die energieimportierenden Volkswirtschaften einem externen Schock ausgesetzt. Die Olpreise stiegen 1973 aufgrund der Mengenverknappung durch das OPEC-Rartell schlagartig an.

Energie hat nur einen Anteil von etwa 5 Prozent am gesamten Input2). Führt die Energieverteuerung aber zu Anpassungsreaktionen, so kann der Produktivitätseffekt wesentlich stärker ausfallen, als die Inputverteuerung allein vermuten läßt. Investitionen zur Energieeinsparung erhöhen den Input, ohne Ausbringungswirkungen $z u$ haben. Energieintensive Anlagen, die weniger oder nicht mehr genutzt werden, bleiben dennoch im Rapitalstock enthalten. Ihr Beitrag zum Output ist aber stark reduziert. Zudem werden viele Investitionsentscheidungen sehr langfristig getroffen. Trotz der Energieverteuerung wurden im Energiesektor Investitionen durchgefüht und hatten dann aufgrund veränderten Nachfrageverhaltens oberkapazitäten zur Folge ${ }^{3}$. Die Energieverteuerung führt somit über die Preiserhöhungen hinaus $\mathrm{zu}$ Kosten durch die erforderlichen Anpassungsreaktionen. Diese verursachen Produktivitātseinbußen, da im Kapitalstock viele Anlagen enthalten sind, mit denen am Markt keine Erträge mehr erwirtschaftet werden können').

1) Vgl. Dicke, H./Hartung, H. (1986), S. $47 \mathrm{ff}$

2) Vgl. Berndt, E, R. (1980), S. $66 f$ und Denison, E.F. (1979), S. $138 f$ sowie Klodt, H. (1984), S. 53

3) Vgl. die Beispiele bei Baily, M.N. (1981), S. 19 und Klodt, H. (1984), S. 57, Fußnote 1

4) Vgl. Baily, M.N. (1981), S. 18f; vgl. auch Jorgenson, D.W. (1984), S. $26 \mathrm{ff}$ und Bruno, M. (1984), S. 10ff, der für das produzierende Gewerbe den Preisanstieg der gesamten Rohstoffe einbezieht. 
Baily verwendet deshalb zur Bestimmung der Faktorproduktivität nicht den Kapitalstock, sondern den jeweils aktuellen Marktwert des Rapitals als Indikator für die "Capital services". Wird die Produktivitätsentwicklung mit Hilfe dieses Ansatzes untersucht, so nimmt der Produktivitätsfortschritt nach 1973 zwar ab, die Abnahme der steigerungsraten beträgt aber im Unternehmenssektor (ohne Finanzsektor) für den Zeitraum 1973 - 1978 nur noch 20 Prozent gegenūber dem Zeitraum 1968 - 1973 , während bei der Bewertung des Rapitaleinsatzes mit dem Buchwert die Wachstumsraten der Produktivität um rund 64 Prozent abnehmen').

Ein letzter Faktor, der bei der Analyse des Produktivitātseinbruchs angesprochen werden soll, ist das Investitionsverhalten. Die Ausgaben für Forschung und Entwicklung blieben seit 1968 real nahezu konstant'). Im gleichen Zeitraum nahm allerdings das Volumen der Anlageninvestitionen bei stark ansteigenden Inflationsraten erheblich $a b^{3}$ ).

Der Beitrag dieser drei Romponenten zum Produktivitätsrūckgang kann nicht quantifiziert werden. Die geringe Investitionstātigkeit der Wirtschaft hat jedoch zur Folge, daß weniger Möglichkeiten $z u$ Innovationen verfolgt werden und daß die Diffusion von Neuerungen verlangsamt wird.

\section{ZUSAMMENFASSUNG}

Ziel dieses Rapitels war es, die Bedeutung der Innovationstätigkeit der Wirtschaft für das Wachstum des Sozialprodukts zu ermitteln. Dazu wird das Wirtschaftswachstum in einzelne Komponenten aufgegliedert.

Eine erste Determinante des Wirtschaftswachstums ist erhöhter Faktoreinsatz. Der Beitrag der Produktinnovationen zum Wachstum des sozialprodukts kann allerdings nicht quantifiziert werden. Untersuchungen der Umsatzstruktur von Unternehmen

1) Vgl. Baily, M.N. (1981), S. $41 \mathrm{ff}$

2) Vgl. fŭr die USA: Denison, E.F. (1979), S. 123 und für die Bundesrepublik Deutschland: Rlodt, H. (1984), S. 89

3) Vgl. Denison, E.F. (1979), S. $50 \mathrm{ff}$ und Klodt, H. (1984), S. 62ff 
zeigen jedoch, daß der Anteil neuer Produkte am Umsatz bedeutend ist.

Verbesserungen der Arbeitsqualität und Reallokationen des Faktors Arbeit waren nach den vorliegenden Informationen die bedeutendsten Quellen des Produktivitätsfortschritts. Mit zunehmendem Bildungsniveau nehmen aber die Möglichkeiten der Produktivitätssteigerung durch verbesserte Ausbildung ab. Auch das Potential der Produktivitätssteigerungen durch Reallokation nimmt mit zunehmendem Ausgleich von sektoralen Produktivitätsunterschieden ab.

Zudem wächst der negative Einfluß staatlicher Interventionen auf die Produktivitätsentwicklung ständig. Für weiteres Wirtschaftswachstum und Produktivitäsfortschritt gewinnt deshalb das Innovationsverhalten, also die Forschungs- und Investitionstātigkeit der Wirtschaft überragende Bedeutung.

Im folgenden sollen nun die Determinanten des Innovationsverhaltens untersucht und die Begründungen für staatliche Interventionen sowie die Möglichkeiten eines Einsatzes des wirtschaftspolitischen Instrumentariums analysiert werden. Ziel der Analyse ist es, Grenzen und Möglichkeiten einer staatlichen Innovationspolitik zu ermitteln. 
c. DETERMINANTEN VON INNOVATIONSENTSCHEIDUNGEN

In diesem Kapitel sollen diejenigen Merkmale herausgearbeitet werden, die für Innovationsprozesse charakteristisch sind. Ausgangspunkt der Diskussion ist ein einfaches neoklassisches Investitionsmodell. Aus der Kritik an diesem Modell werden im weiteren die Besonderheiten von Innovationsprozessen abgeleitet.

I. DAS NEORLASSISCHE INVESTITIONSMODELL

1. Das Grundmodell

Die folgenden Ausführungen zum neoklassischen Investitionsmodell schließen im wesentlichen an Darstellungen von Hall/Jorgenson und Sandmo an').

Zielkriterium für Unternehmensentscheidungen ist danach die Maximierung des Barwerts der Zahlungsüberschūsse aus der Unternehmenstātigkeit bzw. die Maximierung des Barwerts der Dividendenausschüttungen.

Das Investitionsmodell basiert auf folgenden Annahmen: ${ }^{2}$ )

Die Marktpreise werden durch die Aktivitäten des Unternehmens nicht beeinflußt. Sie sind dem Unternehmen auch für die zukünftigen Perioden bekannt. Das Unternehmen kann somit zum gegebenen Marktpreis seinen Output absetzen bzw. die zur Leistungserstellung benötigten Ressourcen (Arbeit, Sachmittel, Rapitall nacheragen.

Der Output (O) wird mit den zwei Produktionsfaktoren Rapital (K) und Arbeit (A) erstellt. Die Produktionsbedingungen seien konstant und gegeben durch die Produktionsfunktion:

1) Hall, R.E./Jorgenson, D.W. (1967), S. $391 \mathrm{ff}$ und Sandmo, A. (1974), S. $287 f f$

2) Vgl. Sandmo, A. (1974), S. 289 
(c.1)

$$
\begin{aligned}
& 0=F(R, L), \\
& F^{\prime}>0, \\
& F^{\prime \prime}<0 .
\end{aligned}
$$

Außerdem wird der gesamte Rapitalstock fremdfinanziert.

Vor Besteuerung läßt sich der Zielzahlungsstrom der Periode $t$ beschreiben durch:

$$
\Pi_{t}=p F\left(R_{t}, L_{t}\right)-w L_{t}-q I_{t}+\delta A-r A_{t},
$$

mit $\pi_{t}$ : Zahlungsüberschuß in Periode $t$,

$K_{t}$ : Rapitalbestand in $t$,

$L_{t}$ : Arbeitseinsatz in $t$,

It : Investitionen in $t$,

$\delta A_{t}$ : Rreditaufnahme in $t$,

At : Fremdkapitalbestand in $t$,

$p$ : Preis pro Outputeinheit,

w : Lohnsatz,

$q$ : Investitionsgüterpreis,

$r$ : zinssatz.

Die Investitionen einer Periode setzen sich zusammen aus Erweiterungs- und Ersatzinvestitionen:

(C.3) $I_{t}=K_{t+1}-K_{t}+d R$, mit d: Abschreibungsquote.

Diese Formulierung impliziert, daß Erweiterungsinvestitionen erst in den Perioden nach der Tätigung der Auszahlung genutzt werden.

Die Rreditaufnahme einer Periode wird zur Finanzierung der Rapitalstockerhöhung verwendet:

$$
\begin{aligned}
\delta A_{t} & =A_{t}+1-A_{t} \\
& =q\left(K_{t+1}-K_{t}\right) .
\end{aligned}
$$


Werden die Gleichungen (C.3) und (C.4) in Gleichung (C.1) eingesetzt, ergibt sich mit $A_{t}=q K_{t}$ :

(C.5) $\pi_{t}=p F\left(K_{t}, L_{t}\right)-w L_{t}-q(r+d) K_{t}$.

Die Zielvorschrift für das Unternehmensverhalten lautet somit:

(C.6) $\quad V=\sum_{t=1}^{\infty}(1+r)-1 \cdot\left[p F\left(R_{t}, L_{t}\right)-w L_{t}-q(r+d) R_{t}\right]=M \operatorname{Max} !$ mit V: Barwert der Zahlungsüberschũsse .

Da in diesem Modell keine (Inter-) Dependenzen zwischen den Perioden bestehen, wird der Barwert der Gewinne optimiert, indem die Zahlungsüberschüsse in jeder einzelnen Periode maximiert werden.

Die Optimierungsbedingungen für Arbeit und Kapital lauten somit:

$$
\begin{aligned}
& \text { (c. 7) } \frac{d \pi_{t}}{d L_{t}}=p \cdot \frac{d F_{L}}{d L_{t}}-w=0, \text { für } t=1, \ldots, \infty, \\
& \text { (C. 8) } \frac{d \pi_{t}}{d R_{t}}=p \cdot \frac{d F_{\mathrm{r}}}{d R_{t}}-q(r+d)=0, \text { für } t=1, \ldots, \infty .
\end{aligned}
$$

Gleichung (C.7) besagt, daß im Optimum das Grenzwertprodukt des Einsatzes einer zusätzlichen Einheit des Faktors Arbeit gleich dem Lohnsatz zu sein hat.

Gleichung (C.8) gibt die Grenzproduktivitätsbedingung für den Einsatz von Kapitalgütern an. Das Grenzwertprodukt des Kapitaleinsatzes muß gleich seinen Opportunitätskosten sein. Diese setzen sich zusammen aus der Verzinsung einer Rapitaleinheit und den Abschreibungen. Der Ausdruck

(c.9) $\quad c=q(r+d)$

gibt somit die Kapitalkosten an. 
Aus Gleichung (C.8) kann auch der Kalkūl eines Investors zum Kauf einer zusätzlichen Rapitaleinheit abgeleitet werden'). Wird das Grenzwertprodukt als "Capital Services" interpretiert, lautet die kritische Bedingung für die Investitionsentscheidung:

$$
\begin{aligned}
q & =\sum_{t=1}^{\infty}(1+t)-t \cdot c \cdot(1+d)-t \\
& =c \cdot(r+d)-1 \text { 2). }
\end{aligned}
$$

D.h. der Barwert der Rapitalerträge bereinigt um die Abschreibungen hat dem Rapitalgüterpreis zu entsprechen.

$\underline{2 .}$ Die Wirkungen der Besteuerung im neoklassischen Investitionsmodell

Nach Einführung einer proportionalen Gewinnsteuer mit dem steuersatz $\tau$ verändert sich die Bedingung (C.10) zu:

$$
\begin{aligned}
q & =\sum_{t=1}^{\infty}(1+r)-t \cdot c \cdot(1-\tau)(1+d)-t+\tau q D, \\
& =\frac{c(1-\tau)}{r+d}+\tau q D,
\end{aligned}
$$

mit D: Barwert der steuerlich anerkannten

Absetzungen pro Rapitaleinheit.

Nach Umformung ergibt sich:

$$
c=q \cdot \frac{(r+d)(1-\tau D)}{1-\tau} .
$$

Die Wirkung der Besteuerung auf die Rapitalkosten und damit auf die Innovationsbereitschaft läßt sich durch Differentiation von Gleichung (C.12) nach dem steuersatz aufzeigen:

1) Vgl. Hall, R.E./Jorgenson, D.W. (1967), S. $392 f$

2) Fưr genügend kleine Werte von $t$ geht $r \cdot d$ gegen null. 
(C.13) $\frac{d c}{d \tau}=q(r+d) \cdot \frac{1-D}{(1-\tau)^{2}}$.

Die Gewinnbesteuerung hat nur dann keinen Einfluß auf die Innovationsentscheidungen, wenn $d c / d \tau=0$, also $D=1$ ist. Eine Erhöhung der Rapitalkosten und damit eine Reduzierung der Innovationsbereitschaft ergibt sich dann, wenn der Barwert der steuerlich zulässigen Absetzungen pro Rapitaleinheit $D<1$ ist.

Zwei Ausgestaltungen der Absetzungsvorschriften führen dazu, daß die Besteuerung keine Verzerrungen verursacht. Zum einen ist der Barwert der Absetzungen $D=1$, wenn die Rapitalgūter sofort abgeschrieben werden und die zinsen nicht abzugsfāhig sind.

Zum zweiten ist die Gewinnbesteuerung dann neutral, wenn die Ertragswertabschreibung und die zinsen auf den Kapitalbestand abgesetzt werden.

$$
\begin{aligned}
D & =\sum_{t=1}^{\infty}(1+r)-t \cdot(r+d) \cdot(1+d)-t \\
& =\frac{r+d}{r+d}=1 .
\end{aligned}
$$

Weichen die steuerlich zulässigen Abschreibungen von der ökonomischen Rapitalentwertung ${ }^{1}$, ab und/oder können nicht die opportunitātskosten des gesamten Rapitalbestands abgesetzt werden, so werden Investitionsentscheidungen durch die Einführung einer Gewinnsteuer verzerrt.

Parameter der steuerlichen Beeinflussung von Investitionsentscheidungen sind der steuersatz sowie die steuerrechtlichen Quoten für die Absetzung von Abschreibungen und zinsen.

1) Zur Ertragswertabschreibung vgl. Wagner, F.W./Dirrigl, H. (1980), S. $36 f f$ und S. $64 f f$ 
Das neoklassische Investitionsmodell baut auf sehr restriktiven Annahmen auf. Diese sind auf die Analyse von Innovationsentscheidungen hin näher zu erörtern'1).

Im dargestellten Modell erfolgen die Investitionsentscheidungen unter sicherheit. Die Preise der Produktionsfaktoren und der Güter sind dem Unternehmen bekannt und werden durch seine Entscheidungen nicht beeinflußt. Zudem ist das Modell in seinen Grundzügen statisch. Die Produktionsbedingungen sind bekannt und bleiben während des Betrachtungszeitraums konstant. Auch bestehen zwischen den Perioden keine (Inter-) Dependenzen.

Tatsächlich erfolgen jedoch Investitions- und insbesondere Innovationsentscheidungen unter Unsicherheit. Diese besteht bezūglich der Absatzpreise und Absatzmengen sowie auch bezūglich des technischen Erfolgs der Innovationsbemühungen. Ein Teil der im Modell als Gewinn ausgewiesenen Größe stellt deshalb in Wirklichkeit eine Risikoprämie dar. Wird sie besteuert, so wird auch die Bereitschaft, risikobehaftete Rapitalinvestitionen zu tätigen, beeinflußt. Auch bei sofortabschreibung bzw. bei Absetzung der gesamten opportunitätskosten des Rapitalbestandes wirkt die Besteuerung unter diesen Bedingungen nicht neutral.

Die Existenz von Unsicherheit hat zudem Auswirkungen auf Rosten und struktur der Kapitalbeschaffung der Unternehmen. Zum einen werden die Rosten des Fremdkapitals mit wachsendem Fremdkapitalbestand zunehmen. Je höher dieser ist, desto größer sind auch die fixen zinszahlungsverpflichtungen. Mit steigendem Fremdkapitalbestand erhöht sich somit die Gefahr der Illiquidität und eines anschließenden Konkurses auch von Unternehmen mit hohem Ertragswert. Deshalb erhōhen die Glãubiger ihre Risikoprämien mit steigendem Kapitalbestand und reduzieren dadurch die Investitionsmöglichkeiten.

Die bisherige Diskussion der Investitionsentscheidungen erfolgte unter der Annahme vollständiger Fremdfinanzierung der Unternehmenstätigkeit. Um den hohen Finanzierungskosten und den damit verbundenen Beschränkungen der Investitionsmöglich-

1) Zur Rritik vgl. Boadway, R.C. (1979), S. $274 f f$ 
keiten zu entgehen, sehen sich die Unternehmen gezwungen, einen Teil ihrer Aktivitäten mit Eigenkapital zu finanzieren. Da die opportunitätskosten des Einsatzes von Eigenkapital steuerlich nicht abgesetzt werden dürfen, verursacht die Besteuerung außer im Fall der sofortabschreibung weitere Verzerrungen').

Außerdem werden kalkulatorische Rosten wie zum Beispiel der Unternehmerlohn oder die Verzinsung des Eigenkapitals steuerlich nicht anerkannt und deshalb als Gewinnbestandteil mitbesteuert.

Im hier besprochenen Modell wird das Optimum durch die Maximierung der periodischen Zahlungsüberschüsse erreicht. In der Realität bestehen jedoch (Inter-) Dependenzen zwischen den Investitionsentscheidungen der einzelnen Perioden. Insbesondere bei Innovationen stehen hohen Anfangsinvestitionen und langen Entwicklungszeiten erst in späteren Perioden entsprechende Einzahlungen gegenüber. Um die zielgröße zu maximieren, sind deshalb zwischenzeitliche Verluste zu finanzieren. Dadurch werden die bereits angesprochenen Kapitalbeschaffungsprobleme weiter verschärt.

Ein zusātzlicher, bisher vernachlässigter Problembereich ergibt sich aus den Interdependenzen, die zwischen der Investitionsentscheidung und der Privatsphäre der Wirtschaftssubjekte bestehen. So führt zum Beispiel die Besteuerung von zinsen dazu, daß die Rapitalgeber, die sich am Nettoertrag ihres Ronsumverzichts orientieren, höhere Zinsen als vor der Besteuerung fordern werden. Dadurch werden die Rapitalkosten erhöht und die Anzahl lohnender Investitionsobjekte wird reduziert.

1) Vgl. Sandmo, A. (1974), S. $295 f$ 
1. Technology-push und Demand-pull

Im einleitenden Rapitel wurde eine Innovation als die erste wirtschaftliche Verwertung einer Neuerung definiert. Diese wurde ihrerseits als neuartige Kombination von Technologie und Bedürfnissen charakterisiert. Der entscheidende Aspekt einer Innovation ist folglich die synthese von (technischem) wissen und Bedūrfnissen'). Eine Innovation besteht somit nicht nur in der Erzeugung und wirtschaftlichen Verwertung neuen (technischen) Wissens, sondern in der neuartigen Verbindung von bekanntem oder neuem technischen wissen mit den Bedürfnissen der Nachfrager.

In der Literatur werden zwei Hypothesen darüber diskutiert, wie innovative Aktivitäten stimuliert werden ${ }^{2}$ ).

Nach der Techology-push-Hypothest sind Wissenschaftler bzw. Forschungsabteilungen der Unternehmen die Initiatoren vus. Innovationen. Ein im Bereich der Grundlagenforschung erreichusr Erkenntnisfortschritt führt zusammen mit bereits bekannten Wissenselementen zu Neuerungen. Diese werden dann von den Unternehmen bzw. deren kaufmännischen Abteilungen kommerzialisiert.

Demgegenüber sind nach der Demand-pull-Hypothese die Marktforschungs- und Produktionsabteilungen die Initiatoren von Produkt- und ProzeBinnovationen. Diese Abteilungen erkennen das Bedürfnis nach einer bestimmten Neuerung und fordern deshalb entsprechende Lōsungsvorschläge von Wissenschaftlern und Forschungsabteilungen.

Zu beiden Hypothesen über die stimulierung von Innovationsaktivitäten werden Beispiele angeführt. Die Laser-Technik wurde in den fünfziger Jahren entdeckt und entwickelt. Verwendungsmöglichkeiten für diese Technik waren damals nicht bekannt. Erst später wurden Anwendungsmöglichkeiten gefunden - von der Chirurgie bis zum Rrieg der sterne ${ }^{3}$ ).

1) Vgl. Utterback, J.M. (1979a), S. 46

2) Vgl. Schmockler, J. (1966), S. 87 ff und Ramien, M.I./Schwartz, N.L. (1982), S. $33 f f$

3) Vgl. Kamien, M.I./Schwartz, N.L. (1982), S. 34 
Auf der anderen seite wurde nach industriellen Produktionsverfahren für Cortison erst geforscht, nachdem die Wirksamkeit dieses Medikaments bei der Behandlung von Arthritis bekannt war').

Werden die beiden Hypothesen kritisch betrachtet, so ist es zweifelhaft, ob sie als konkurrierende Ansätze zur Erklärung von Innovationsaktivitäten herangezogen werden können.

Jede unternehmerische Aktivităt ist auf Gewinnerzielung ausgerichtet. Produktionstätigkeit setzt stets die Existenz von (latenter) Nachfrage voraus, die vom Unternehmer erkannt wird. Nur wenn ein Unternehmer solche bisher unbefriedigt gebliebenen Bedürfnisse erkennt, wird er sich in angewandter Forschung engagieren. Sie ist definiert als die Forschungstātigkeit, die in Erwartung von Pay-offs in bestimmten Bereichen unternommen wird '). Die angewandte Forschung greift jedoch ihrerseits auf die Ergebnisse der Grundlagenforschung zurūck. Technology-push und Demand-pull sind somit Teile eines einzigen Prozesses. Sie sind keine konkurrierenden Erklärungsansātze für die stimulierung innovativer Aktivitāten ${ }^{3}$ ). Bei entsprechender Ausdehnung des Betrachtungszeitraums kann jedes Ergebnis angewandter Forschung auf einen Erkenntnisfortschritt im Bereich der Grundlagenforschung zurūckgeführt werden. Wird die hier skizzierte Betrachtungsweise auf die oben angeführten Beispiele übertragen, so läßt sich in beiden Fällen die gleiche struktur des Innovationsprozesses erkennen.

Am Beginn beider Innovationsprozesse stehen Ergebnisse der Grundlagenforschung: zum einen die Entdeckung des Laserstrahls, zum anderen die Entwicklung des Wirkstoffes Cortison. Die innovative Leistung besteht in der Kombination bisher unbefriedigter Nachfrage mit den neuen technologischen Möglichkeiten. Die angewandte Forschung mit anschließender Rommerzialisierung setzt in beiden Fällen erst ein, nachdem die Marktchancen für die technischen Möglichkeiten bekannt sind.

1) Vgl. Kamien, M.I./Schwartz, N.L. (1982), S. 35

2) Vgl. Rosegger, G. (1980), Kap. 1

3) Ahnlich Freeman, C. (1979), S. $213 f$ 
Im folgenden Abschnitt wird der idealtypische Verlauf von Innovationsprozessen auf einem neu erschlossenen Markt dargestellt. Im Anschluß daran werden die besonderen Dimensionen von Innovationen unter Bezug auf das neoklassische Investitionsmodell herausgearbeitet.

$\underline{2 .}$ Der Ablauf von Innovationsprozessen

Ausgangspunkt der folgenden Ausführungen sind Aktivitäten, Verhalten und Umgebung einer "Productive Unit". Sie wird verstanden als eine Unternehmenseinheit, die eine homogene Produktlinie herstellt und anbietet. Eine "Productive Unit" oder Unternehmenseinheit kann somit ein Ein-Produkt-Unternehmen oder ein Produktbereich eines Mehr-Produkt-Unternehmens sein').

Auslöser der Tätigkeit einer Unternehmenseinheit ist eine Innovation' ${ }^{2}$, verstanden als eine neuartige Kombination von Bedürfnissen mit Gütern und/oder Dienstleistungen, die deren Befriedigung dienen sollen.

Diese erste Phase der unternehmerischen Aktivität ist mit großen Unsicherheiten verbunden ${ }^{3}$ ). Unsicherheit besteht bezüglich des technischen und des marktlichen Erfolgs. Fraglich ist zum einen, ob die Produkteigenschaften so ausgestaltet werden können, daß sie dem Anforderungsprofil entsprechen, welches das Unternehmen aufgrund der erkannten Bedürfnisse entwickelt hat. Zum zweiten besteht Unsicherheit darüber, ob die vermuteten bzw. ermittelten Bedürfnisstrukturen in kaufkräftige Nachfrage umgesetzt werden, wenn die entsprechenden Güter am Markt angeboten werden.

Dominierend in der Anlaufphase der Unternehmenstätigkeit ist das Bestreben, die Funktionalität der Produkte zu verbessern. Auch Spezialanfertigungen nach Kundenwünschen werden gemacht, um zunächst in Marktnischen Fuß zu fassen.

sind erste Marktsegmente durch diese grundlegenden (major, radical) Produktinnovationen erschlossen, versucht die Unter-

1) Vgl. Utterback, J.M. (1979b), S. $136 f$

2) Zum Folgenden vgl. Utterback, J.M. (1979b), S. $137 f f$ und derselbe (1977), S. $12 \mathrm{ff}$

3) Vgl. Freeman, C. (1979), S. 213 
nehmenseinheit, ihr Produktionsvolumen durch die Anpassung ihres erfolgreichen Produkts an die Ansprüche zusätzlicher Abnehmergruppen auszudehnen. Dazu werden kleinere (minor, incremental) Produktinnovationen in Funktionalität und Design vorgenommen. Da zu diesem Zeitpunkt die Märkte bereits relativ gut erschlossen sind, sind die Unsicherheiten bezüglich des Markterfolgs vergleichsweise gering.

Eine ähnliche Verlagerung der Bemühungen von grundlegenden zu inkrementalen Innovationen ist auch auf der Produktionsseite festzustellen.

Zu Beginn der Aktivitäten ist der Produktionsprozeß flexibel organisiert. Der Einsatz qualifizierter Arbeitskräfte und vielseitig einsetzbarer Anlagen ermöglicht schnelle und wenig kostenaufwendige Umstellungen. Mit der Ausdehnung des Produktionsvolumens ändert sich auch die struktur der Produktionstätigkeit. Durch eine Verlagerung der Innovationsaktivitäten auf den Produktionsprozeß können beträchtliche Kosteneinsparungen erreicht werden. Sie resultieren aus einer Erhöhung der Arbeitsteilung und einer Intensivierung des Rapitaleinsatzes. Dadurch wird jedoch die Produktionsstruktur inflexibler und mit hohen Fixkosten belastet, sodaß diese Unternehmen ihrerseits wieder verwundbar durch kleine, stark marktorientierte Unternehmen werden, die zunächst in Marktlücken drängen'1).

Gleichzeitig wird versucht, eine standardisierung der Produkte zu erreichen. Diese Bestrebungen führen zu einer Reduzierung des Sortiments auf eines oder wenige Grundmodelle und verschiedene Produktvarianten.

Die weitere Innovationstätigkeit beschränkt sich auf wenige inkrementale, vorwiegend kostenorientierte Innovationen, in die auch neu erkannte technische Möglichkeiten eingehen. Sie erfordern in der Regel eine Anpassung von Produkt und Produktionsverfahren.

1) Vgl. Abernathy, W.J./Utterback, J.M. (1982), S. 100 

kurrenzbeziehungen

In der bisherigen Analyse werden Ronkurrenzbeziehungen nicht berücksichtigt. Der Innovationsablauf entspricht dem Fall einer Unternehmenseinheit, die sich durch ihre Produktinnovation eine Monopolstellung geschaffen hat und keine potentielle Konkurrenz befüchten muß. Ist die Innovation ertragreich, dann ist eine solche Unternehmenssituation allerdings nur kurzfristig denkbar. Tatsächlich mūssen die Unternehmen bei ihren Entscheidungen immer den Markteintritt weiterer Unternehmen sowie das Verhalten potentieller Konkurrenten beachten.

Bei der Einbeziehung von Ronkurrenzverhältnissen in die Analyse müssen zwei Konstellationen unterschieden werden.

Die innovative Unternehmenseinheit kann zum einen mit einem Substitutionsgut in einen bereits erschlossenen Markt drängen. Zum zweiten kann das Unternehmen durch seine Innovationsanstrengungen einen neuen Markt erschließen. Bei entsprechenden Gewinnmöglichkeiten muß es jedoch mit nachstoßendem Wettbewerb anderer Unternehmen rechnen. Die Existenz (potentieller) Ronkurrenz führt eine weitere Unsicherheitskomponente in die Innovationsentscheidung ein. Zusätzlich zu den Unsicherheiten über den technischen und den marktlichen Erfolg beeinflußt die Unsicherheit über die Reaktionen der Ronkurrenten das Innovationsverhalten.

Tritt die innovative Unternehmenseinheit mit einem substitutionsprodukt auf den Markt, so besteht fūr die etablierten Unternehmen die Gefahr von Marktanteilsverlusten, die zu ihrem Ausscheiden aus dem Markt führen können'1). Sie werden deshalb versuchen, ihre Produkte durch erhōhte Anstrengungen zu verbessern. Gelingt es dem innovativen Unternehmen trotz der Abwehrreaktionen der etablierten Anbieter, aufgrund der neuartigen struktur seines Produkts auf Teilmärkten Fuß zu fassen oder zusätzliche verwendungsmöglichkeiten zu erschließen, so steht ihm durch inkrementale Innovationen ein wesentlich größeres Potential zur Verbesserung der Produktqualität und zu Rostensenkungen zur Verfügung.

1) Zum Folgenden vgl. Utterback, J.M. (1979a), S. $55 \mathrm{ff}$ 
Die Möglichkeiten der Produktinnovation auf der Basis der alten Technologie sind für die etablierten Unternehmen in der Regel begrenzt. Werden, um die Marktanteile zu halten, Preissenkungsstrategien verfolgt, so stehen dem innovativen Unternehmen zumeist wesentlich größere Rostensenkungsmöglichkeiten zur Verfügung, da es über eine standardisierung der Produktion Kostenvorteile erzielen kann, die von den etablierten Anbietern bereits ausgeschöpft sind. Im Lauf des Innovationsprozesses kommt es somit zu einer Verbesserung der Produkte der etablierten Unternehmen bei einem niedrigeren Preisniveau. Diese inkrementalen Innovationen reichen jedoch in der Regel nicht aus, die Vorteile der grundlegenden Produktinnovation $\mathrm{zu}$ kompensieren. Je höher allerdings die Gewinnspanne und je homogener die Gruppe der etablierten Anbieter, desto größer ist der Druck, den sie auf das innovative Unternehmen, zum Beispiel durch vorübergehende Dumpingpreisaktionen, ausüben kōnnen. Bei entsprechend aggressivem Verhalten der etablierten Unternehmen kann das innovative Unternehmen dadurch zur Aufgabe gezwungen werden').

Wenn durch die Innovation eine neuer Markt erschlossen wird, wirken sich die Konkurrenzbeziehungen vor allem auf den zeitlichen Ablauf des Innovationsprozesses aus. Wird dem Unternehmen während der Vorbereitung des Markteintritts bekannt, daß ein Konkurrent dasselbe Innovationsziel verfolgt, so mu es entscheiden, ob es seinen Markteintritt vorzieht, um als erster Anbieter den Markt prägen zu können, oder ob es den Markteintritt des Ronkurrenten abwartet und dann versucht, die "Rinderkrankheiten" bei seinem Produkt zu vermeiden.

Ist die innovative Unternehmenseinheit bereits am Markt und treten dann Imitatoren ("statische Wirte"2)) auf, so wird der Druck, die produkte in Funktionalität und Design an die Nachfragerbedürfnisse anzupassen, erhöht. Zudem erhōht sich auch der Rostendruck. Die Unternehmen versuchen, unter Ausweitung

1) Zu den möglichen Reaktionen der etablierten Unternehmen vgl. Bain, J.S. (1956), S. $97 f$

2) Zur Unterscheidung von Virten und Unternehmern vgl. Schumpeter, J.A. (1964), S. 119ff 
der Rapazitäten den Produktionsprozeß zu standardisieren. Dieser Prozeß führt dann auch auf expandierenden Märkten zu Oberkapazitäten und zum Ausscheiden von Unternehmen, die nicht durch permanente Produktinnovationen standig neue Räuferschichten erschließen können'l).

\section{DIE BESONDERHEITEN VON INNOVATIONSENTSCHEIDUNGEN}

Im neoklassischen Investitionsmodell wird das zielkriterium, die Maximierung des Barwerts der Ausschüttungen, durch die Erfüllung der Grenzproduktivitätsbedingungen in jeder Periode optimiert. Da in diesem Modell konstante Produktionsbedingungen unterstellt werden, zudem die Marktbedingungen vom Unternehmen nicht beeinflußt werden können und auch die Produktionsentscheidungen interperiodisch unverbunden sind, führt das neoklassische Modell praktisch zu einem einperiodischen, statischen Ansatz.

Bei Innovationsentscheidungen bestehen dagegen starke Interdependenzen zwischen den Aktivitäten und Aktionsmöglichkeiten der verschiedenen Wettbewerber und zwischen verschiedenen Perioden. So werden zum Beispiel die Gestaltung des Markteintritts und die Vornahme erster Produktanpassungen weitgehend durch die vorbereitenden Aktivitäten in frūheren Perioden determiniert. Zudem sind sowohl die Technik als auch die Marktbedingungen während des Innovationsprozesses nicht konstant. Die sich ändernden ökonomischen Bedingungen und die Notwendigkeit der Betrachtung größerer Entscheidungszeiträume führen Unsicherheiten in die Innovationsentscheidung ein. Die Unsicherheit hat zur Folge, daß die Prämisse des vollkommenen Rapitalmarkts aufgegeben werden muß. $\mathrm{Zu}$ untersuchen sind die Möglichkeiten und Risiken der Aufbringung von Risikokapital sowie ihre Ronsequenzen für die Innovationsentscheidung.

1) Vgl. die Mărkte für elektronische Bauteile und Personal- bzw. Heimcomputer 
Außerdem beeinflussen steuerliche Institutionen die Innovationsentscheidung und damit auch die Bereitschaft zur Risikoübernahme. Insbesondere Verlustausgleichs- und Abschreibungsmöglichkeiten haben aus der Sicht des Investors Auswirkungen auf das mit den einzelnen Handlungsalternativen verbundene Risiko und damit auf ihre Realisierung.

IV. ZUSAMMENFASSUNG

In diesem Rapitel wurden die grundlegenden Merkmale von Innovationsentscheidungen untersucht. Ausgangspunkt der Diskussion war ein einfaches neoklassisches Investitionsmodell, in das die Wirkungen der Besteuerung einbezogen wurden. Diesem Investitionsmodell wurde der idealtypische Verlauf von Innovationsprozessen gegenübergestellt. Zunächst wurde die Erschließung eines neuen Marktes durch einen Pionierunternehmer untersucht. Die Darstellung der Innovationsprozesse wurden durch die Analyse der Einführung einer Innovation auf einem bereits erschlossenen Markt ergänzt. Aus der Gegenüberstellung dieser beiden Ansätze wurden die Besonderheiten von Innovationsentscheidungen abgeleitet. Es zeigt sich, daß das neoklassische Investitionsmodell zwar zur Analyse von Entscheidungen über Ersatz- und auch Erweiterungsinvestitionen auf bereits erschlossenen Mārkten mit ausgereiften Produkten und relativ konstanter Marktstruktur und Nachfrage eingesetzt werden kann, zur Untersuchung von Innovationen jedoch nicht geeignet ist. Die wirkungen steuerlicher Institutionen auf die Bereitschaft zur Risikoūbernahme sowie die Finanzierungsund Wettbewerbsbedingungen sind von besonderer Bedeutung für die Innovationsbereitschaft. Diese werden im folgenden eingehend analysiert. 
D. DER EINFLUSS DER MARKTVERHALTNISSE AUF DIE INNOVATIONSBEREITSCHAFT

In diesem Kapitel wird der Einfluß der Marktverhältnisse auf die Innovationsaktivitäten der Unternehmen und das Innovationstempo untersucht. Im Zentrum der Analyse steht die Entscheidung über den Zeitpunkt des Markteintritts, also der geplante Innovationszeitpunkt. Die Untersuchung in diesem Rapitel hat zum ziel, die Bedeutung der Wettbewerbsbedingungen für Ausmaß und Intensität der Innovationsanstrengungen $z u$ bestimmen. Zunãchst wird nach einer Diskussion der Eigenschaften von Innovationskostenfunktionen der optimale zeitplan zur Durchführung eines Innovationsvorhabens für ein Unternehmen abgeleitet, das nicht in Ronkurrenzbeziehungen steht. In den darauffolgenden Abschnitten wird dieser Ansatz durch die Einbeziehung von Innovationskonkurrenz erweitert.

\section{I. DIE INNOVATIONSPLANUNG OHNE RONRURRENZBEZIEHUNGEN}

1. Die Innovationskosten

Als Rosten eines Innovationsvorhabens werden sãmtliche Aufwendungen verstanden, die bis zur Markteinführung des neuen Produkts bzw. bis zum Einsatz der Prozeßinnovation anfallen. Neben den Ausgaben für Forschung und (experimentelle) Entwicklung sind dies auch die Aufwendungen für Ronstruktion und Design sowie die Kosten der Produktions- und Absatzvorbereitung' ' Für die Gestaltung der Innovationsplanung und insbesondere die Wahl des Innovationszeitpunktes ist es wichtig zu wissen, welche Wirkungen von einer Verkürzung oder Verlängerung des Innovationszeitraums auf die Kosten des Vorhabens ausgehen' ${ }^{2}$.

1) Vgl. Schmalholz, В./Scholz, В. (1987), S. 24

2) Zum Folgenden vgl. Scherer, F.M. (1966), S. $71 \mathrm{ff}$ und derselbe (1967), S. $359 f f$ 
Die Phase vom Abschluß der Innovationsplanung bis zur Markteinfūhrung bzw. bis zur Fertigstellung des Projekts ist dadurch gekennzeichnet, daß in Trial-and-Error-Prozessen Mittel und Verfahren gefunden werden müssen, welche die Realisierung der angestrebten Innovation ermöglichen. Eine Verkürzung des Innovationszeitraums hat zur Folge, daß die bis zum Erfolg erforderliche Anzahl von Versuchen innerhalb kürzerer zeit durchzuführen ist. D.h. daß einzelne Forschungs- und Entwicklungsansätze nicht mehr nacheinander verfolgt werden können, sondern parallel angegangen werden müssen. Während jedoch bei sequentiellem Forschungsverlauf Erkenntnisse aus Fehlschlägen in späteren Versuchen verwertet und so Wiederholungen gleicher Fehler vermieden werden können, beginnt bei paralleler Forschung jeder Versuch auf dem gleichen Informationsstand. Lerneffekte können somit nicht erzielt werden'1).

Desweiteren kōnnen bei sequentiellem Vorgehen die Forschungsanstrengungen sofort gestoppt werden, wenn ein erfolgreicher Ansatz gefunden ist. Nur wenn erst der letzte der geplanten Versuche zum Erfolg führt, sind bei sequentiellem und parallelem Forschen die gleiche Anzahl von Versuchen erforderlich. Dieser Fall ist jedoch unwahrscheinlich, da bei Forschungsund Entwicklungsaktivitäten Lerneffekte erzielt werden können.

AuBerdem zwingt das parallele Vorgehen zu einer Erhöhung des Personal- und Mitteleinsatzes. Diese ist, sobald das Angebot an Produktionsfaktoren bei konstanter Qualität nicht völlig elastisch ist, mit abnehmenden Grenzerträgen verbunden ${ }^{2}$ ).

Eine Verkürzung der Projektdauer führt somit zu steigenden Kosten, da Lerneffekte nicht genutzt werden können, der Erwartungswert der Anzahl von Trial-and-Error-Prozessen erhöht wird und da eine Intensivierung des Mitteleinsatzes nur zu sinkenden Grenzerträgen möglich ist.

zur Bestimmung des optimalen Innovationszeitpunkts $T$ wird deshalb eine Rostenfunktion mit den folgenden Eigenschaften unterstellt:

1) Vgl. Nelson, R.R. (1961), S. $352 \mathrm{f}$

2) Vgl. Nelson, R.R. (1961), S. 362 
(D.1)

$$
\begin{aligned}
& C(T)>0, \\
& C^{\prime}(T)<0, \\
& C^{\prime \prime}(T)>0, \text { für alle } T>0 .
\end{aligned}
$$

D.h. bei stets positiven Entwicklungskosten führt eine Verlängerung des Innovationszeitraums zu überproportionalen Kostenersparnissen').

\section{Der Innovationskalkül}

Um den Kalkül zur Bestimmung des optimalen Innovationszeitpunktes aufstellen zu können, muß zunächst der strom der Erträge aus der Innovationstätigkeit bestimmt werden. Für den Ertragsstrom wird angenommen, daß der Innovator bei sofortiger Realisierung seines Projekts im Zeitpunkt $t=0$ den Betrag Po abschöpfen kann' '). Dieser geht über den marktüblichen Ertrag hinaus und wird deshalb als Quasi-Rente des Innovators bezeichnet. Im Zeitablauf ändern sich die Erträge mit der Rate $g$ :

(D.2) $\quad P_{0}(t)=e^{g t} P_{0}$.

Ist g>0, sieht sich der Innovator einem wachsenden Markt gegenüber ${ }^{3}$, bei $g<0$ befindet sich der Innovator auf einem schrumpfenden Markt4).

Wird mit dem zinssatz $r$ diskontiert, beträgt der Barwert der erwarteten Erträge bei einer Vornahme der Innovation im Zeitpunkt $\mathbf{T}$ :

(D. 3)

$$
\begin{aligned}
V(T) & =\int_{s=t}^{\infty} e^{-r s} P_{0}(s) d s \\
& \left.=e^{-(r-g) t} P_{0} /(r-g) s\right) .
\end{aligned}
$$

1) Vgl. Kamien, M.I./Schwartz, N.L. (1972), S. 50

2) Zum Folgenden vgl. Kamien, M.I./Schwartz, N.L. (1972), S. $45 f f$

3) So zum Beispiel Barzel, Y. (1968), S. 349

4) Diesen Fall nimmt Scherer, F.M. (1967), S. $367 f$ an.

5) Da nur endliche Gewinne okonomisch sinnvoll sind, soll gelten: $g<r$. 
Ist der Unternehmer in seinen Entscheidungen von Konkurrenten unabhängig, weil er beispielsweise in einem regulierten Markt mit eintrittsverhindernden schranken aktiv ist, reduziert sich sein Kalkül darauf, den Ertragswert seiner Gewinne zu maximieren.

Mit

$$
R(t)=V(t)-C(t) \Longrightarrow \operatorname{Max} !
$$

sind die Bedingungen erster und zweiter ordnung:

(D.5)

$$
R^{\prime}(t)=0
$$

und

$$
R^{\prime \prime}(t)<0
$$

zu erfüllen;

d.h.

$$
-e^{-(r-g) T} P_{0}=C^{\prime}(T)
$$

und

$$
(r-g) e^{-(r-g) T P_{0}}-C^{\prime \prime}(T)<0 .
$$

Die Bedingung erster Ordnung besagt, daß im Optimum der Anstieg des Barwerts der Erträge bei einer Verkürzung des Innovationszeitraums um eine Zeiteinheit gleich dem Kostenanstieg aufgrund der Zeitverkürzung sein muß.

Aus der Marginalbedingung ist auch zu entnehmen, daß das Innovationsprojekt um so stärker forciert wird, je höher die erwartete Quasi-Rente und die Wachstumsrate sind und je geringer der Diskontierungssatz ist.

Da eine Verkürzung der Entwicklungszeit nur zu überproportional steigenden Kosten möglich ist, ist auch die Bedingung zweiter ordnung in der Regel erfüllt, je eher, um so stärker der Markt wächst.

Dieser Kalkül zur Bestimmung des optimalen Innovationszeitpunkts kann nach der Vornahme einiger Ergänzungen auch eingesetzt werden, um das Innovationsverhalten auf Ronkurrenzmärkten zu analysieren.

Bevor die Untersuchung unter diesem Aspekt fortgesetzt wird, soll anhand der graphischen Darstellung des Innovationskalküls auf einen Punkt eingegangen werden, der für die Interpretation der nachfolgenden Untersuchungen wichtig ist. 
Abbildung D.1: Innovationskalkül

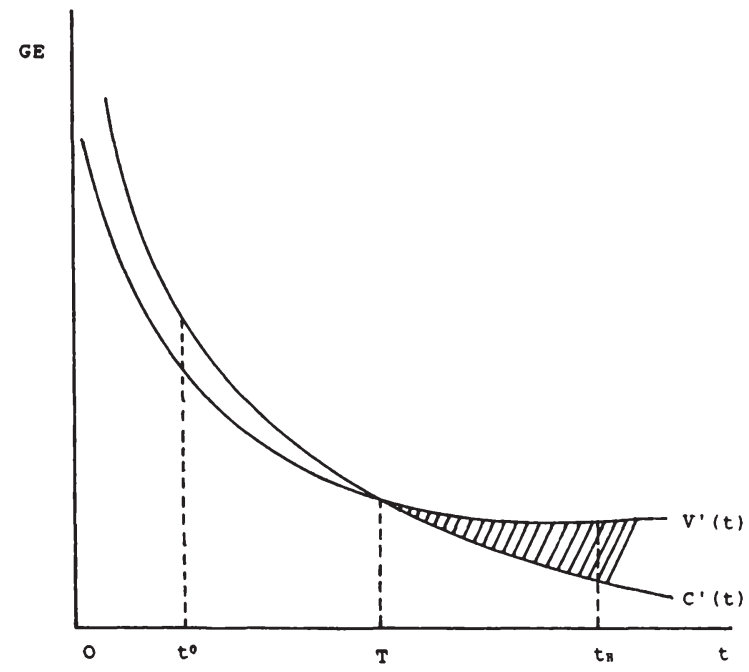

In Abbildung D.1 sind die Grenzerlös- und die Grenzkostenkurve für ein Innovationsprojekt dargestellt. Der optimale Innovationszeitpunkt $T$ wird durch den schnittpunkt der beiden kurven bestimmt. Im zeitpunkt $T$ wird der maximale Innovationsgewinn realisiert. Die schraffierte Fläche zwischen den beiden Kurven rechts von $T$ stellt den Rapitalwert des Projekts dar. Links von $T$ sind die Rosten der Innovation pro Zeiteinheit höher als die Erträge. Wird die Innovation trotzdem früher als in $T$ durchgeführt, reduziert sich ihr Rapitalwert. Für den Innovator ist das Projekt solange noch attraktiver als die durch die Diskontierungsrate reprāsentierte Alternativanlage, als die Fläche zwischen den beiden Kurven links von $T$ nicht größer wird als die Fläche rechts von $T^{11}$. Gesamtwirtschaftich ist das Vorziehen des Innovationszeitpunkts suboptimal. Solange allerdings das Projekt für den Innovator profitabel bleibt, bewirkt seine Realisierung auch gesamtwirtschaftlich eine Wohlfahrtssteigerung. Sie weicht vom sozialen optimum im Ausmaß der Fläche links von $T$ ab.

1) Vgl. Barzel, Y. (1968), S. 351 
Staatliche Interventionen, die eine Erhöhung der Innovationsgewinne bewirken, führen im einzelwirtschaftlichen Ralkūl zu einer Beschleunigung der Innovationsanstrengungen, die aber gesamtwirtschaftlich eine Wohlfahrtsminderung bedeutet.

II. DIE INNOVATIONSENTSCHEIDUNG UNTER WETTBEWERBSBEDINGUNGEN

1. Der entscheidungstheoretische Ansatz

1.1 Das Mode11

Der im vorangehenden Abschnitt aufgestellte Ralkül zur Bestimmung des optimalen Innovationszeitpunkts soll hier durch die Berücksichtigung von Konkurrenzbeziehungen ergänzt werden'1).

Bezieht das innovationsbereite Unternehmen die Aktivitāten seiner (potentiellen) Ronkurrenten in seine Planungen ein, so kann das Unternehmen nicht mehr davon ausgehen, daß es bei erfolgreicher Realisierung des Innovationsprojekts die gesamte Quasi-Rente aus der Innovationstätigkeit abschöpfen kann. Zum einen muß das Unternehmen damit rechnen, daß ihm ein Konkurrent zuvorkommt und es selbst nur noch Imitationsgewinne erzielen kann. Zum zweiten hat das Unternehmen zu berücksichtigen, daß, auch wenn es vor der Konkurrenz mit seiner Innovation auf den Markt kommt, sein Innovationsgewinn durch das Auftreten von Imitationswettbewerb reduziert wird.

Folgende Annahmen werden über die Hōhe der erwarteten Gewinne getroffen:

$$
V(t)=\left\{\begin{array}{l}
e^{-(r-g) t} \cdot P_{0} \text { für } T \leq t<v \\
e^{-(r-g) t} \cdot P_{1} \text { für } T<v \leq t \\
e^{-(r-g) t} \cdot P_{2} \text { für } v<T \leq T \\
0 \leq P_{1} \leq P_{0} ; 0 \leq P_{2} \leq P_{0} ; P_{1} \stackrel{\geqslant}{=} P_{2},
\end{array}\right.
$$

1) Zum folgenden Modell vgl. Kamien, M.I./Schwartz, N.L. (1972), (1974) und (1976), sowie Barzel, Y. (1968). 
mit

T: Geplanter Innovationszeitpunkt des Unternehmens,

$t$ : Betrachtungszeitpunkt,

v: Zeitpunkt des Markteintritt eines Ronkurrenten.

Der Gewinn beträgt $\mathrm{P}_{0}$, wenn das Unternehmen einziger Anbieter auf dem Markt, also Monopolist ist. Er geht auf $P_{1}$ zurück, wenn das Unternehmen zwar Innovator ist, Ronkurrenten jedoch Imitationswettbewerb betreiben. Der Gewinn sinkt schließlich auf $\mathrm{P}_{2}$, wenn dem Unternehmen ein Ronkurrent mit der Innovation zuvorkommt und es nur noch als Imitator auftreten kann.

Ober die Wahrscheinlichkeit des Auftretens von Konkurrenten bildet sich das Unternehmen folgende subjektive Verteilungsfunktion:

(D.9)

$$
F(v)= \begin{cases}1-e^{-b v}, & \text { für } 0 \leq v<T \\ 1-e^{(k-b) T-k v,} & \text { für } v \geq T\end{cases}
$$

Der erste Fall gibt die Wahrscheinlichkeit dafür an, daß dem Unternehmen ein Ronkurrent mit der Innovation zuvorkommt und im zweiten Fall wird die Wahrscheinlichkeit für das Auftreten von Imitatoren, nachdem das Unternehmen innoviert hat, ausgedrückt.

Durch die Parameter $h$ und $k$ wird die Wettbewerbsintensität bestimmt. Je höher diese beiden Werte sind, desto größer ist der Wert der kumulierten Wahrscheinlichkeitsfunktion $F(v)$ für das Auftreten eines Konkurrenten als Innovator bzw. Imitator. Die Erwartungswerte für das Auftreten eines Ronkurrenten betragen:

(D.10) $\quad E(v)= \begin{cases}1 / h \quad \text { für } 0 \leq v<T \\ T+1 / k, & \text { für } v \geq T 11 .\end{cases}$

D.h. ist bisher kein Konkurrent aufgetreten, beträgt die Wahrscheinlichkeit, daß dies im nächsten Moment $\delta t$ geschieht,

1) $\operatorname{Mit} E(v)=\frac{1-F(v)}{F^{\prime}(v)}$ 
h bzw. bei Imitation im Zeitpunkt ( $T+\delta t) k$. Diese Eintrittswahrscheinlichkeiten sind also über den gesamten Betrachtungszeitraum konstant'). Im Zeitraum vor dem Betrachtungszeitpunkt liegende Ereignisse und Prozesse gehen nicht in die Eintrittswahrscheinlichkeit ein, die Wahrscheinlichkeitsverteilung ist "memoryless".

Tatsächlich dürfte sich aber die Wahrscheinlichkeit des Markteintritts eines Ronkurrenten mit zunehmendem zeitablauf erhöhen'). Auch wird diese Wahrscheinlichkeit nicht unabhängig von der Anzahl der im Wettbewerb aktiven Unternehmen sein $^{3}$ ). Im folgenden wird trotz dieser Mängel die analytisch leichter handhabbare Exponentialverteilung unterstellt, da die resultierenden Ergebnisse auch bei der Verwendung einer erweiterten Verteilungsfunktion gültig sind4).

Mit diesen Ergänzungen, den Erträgen bei verschiedenen Marktkonstellationen und deren jeweiligen Wahrscheinlichkeiten, ist es möglich, den kapitalwertmaximierenden Innovationszeitpunkt in einem Marktmodell zu bestimmen.

Die erwarteten Erträge aus dem Innovationsprojekt belaufen sich auf:

$$
\begin{aligned}
V(T)= & \int_{t=T}^{\infty} e^{-(r-g) t} \cdot\left\{P_{0}[1-F(t)]\right. \\
& \left.-P_{1}[F(t)-F(T)]+P_{2} \cdot F(T)\right\} d t .
\end{aligned}
$$

Dabei ist [1-F(t)] die Wahrscheinlichkeit dafür, daß das Unternehmen bis zum Zeitpunkt $t$ Monopolist ist, [F(t)-F(T)] die Wahrscheinlichkeit dafür, daß das Unternehmen zwar Innovator ist, jedoch bis zum Zeitpunkt $t$ Innovatoren aufgetreten sind und $F(T)$ ist die Wahrscheinlichkeit dafür, daß dem Unternehmen ein Konkurrent mit der Innovation zuvorkommt und es nur noch Imitationsgewinne erzielen kann.

Einsetzen von Gleichung $(D .10)$ in (D.11) und Umformen ergibt:

1) Vgl. Kamien, M.I./Schwartz, N.L. (1972), S. $46 f$

2) Vgl. Kamien, M.I./Schwartz, N.L. (1980), S. 245

3) Vgl. Fethke, G.C./Birch, J.J. (1982), S. $276 \mathrm{ff}$

4) Vgl. Kamien, M.I./Schwartz, N.L. (1980), S. $247 f$

und Fethke, G.C./Birch, J.J. (1982), S. $276 \mathrm{ff}$ 
(D.12)

$$
\begin{aligned}
V(T)= & \int_{t=1}^{\infty}\left[e^{-(r-g+k) t+(k-t) T} \cdot\left(P_{0}-P_{1}\right)\right. \\
& +e^{\left.-(r-g) t-k T \cdot\left(P_{1}-P_{2}\right)+e^{-(r-g) t} \cdot P_{2}\right] d t .}
\end{aligned}
$$

Nach Vornahme der Integration ergibt sich die Erlösfunktion in Abhängigkeit vom Innovationszeitpunkt:

$$
\begin{aligned}
& V(T)=e^{-D T} \cdot e^{-(r-g) T} \cdot\left[\frac{P_{0}-P_{1}}{r-g+k}+\frac{P_{1}}{r-g}\right] \\
& +\left(1-e^{-b T}\right) \cdot e^{-(r-g) T} \cdot \frac{P_{2}}{r-g} .
\end{aligned}
$$

Der erste summand gibt den erwarteten Barwert der Erträge als Innovator, der zweite den erwarteten Barwert als Imitator an. Die jeweiligen Wahrscheinlichkeiten betragen $e^{-\Delta T}$ bzw. $\left(1-e^{-b T}\right)$.

Das Optimierungsproblem für die Wahl des Innovationszeitpunkts lautet:

$$
R(T)=V(T)-C(T) \Longrightarrow M a x !
$$

Durch Differentiation nach $T$ ergeben sich die Bedingungen erster und zweiter ordnung:

$$
\begin{aligned}
V^{\prime}(T)= & -(r-g+h) e^{-(r-g+b) T} \cdot\left[\frac{P_{0}-P_{1}}{r-g+k}+\frac{P_{1}-P_{2}}{r-g}\right] \\
& -e^{-(r-g) T \cdot P_{2}-C^{\prime}(T)=0}
\end{aligned}
$$

und

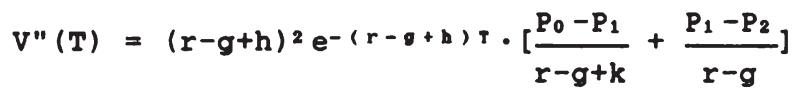

$$
\begin{aligned}
& +(r-g) e^{-(r-g) T \cdot P_{2}-C^{\prime \prime}(T)<0 .}
\end{aligned}
$$

Besteht keine Ronkurrenz, also $h=k=0$, sind $\mathrm{P}_{0}=\mathrm{P}_{1}$ und $\mathrm{P}_{2}=0$. Nach Einsetzen dieser Werte in die Bedingung erster ordnung ergibt sich als spezialfall dieses erweiterten Modells die im vorigen Abschnitt abgeleitete Marginalbedingung für die Wahl 
des optimalen Innovationszeitpunkts'). Ein Beispiel für diese Ronstellation ist der Fall der Rartellbildung mit gleichzeitiger Errichtung von Marktschranken bei gemeinsamer Durchführung des Innovationsprojekts durch die Rartellmitglieder. Die durch das Konkurrentenverhalten ausgelösten Unsicherheiten sind eliminiert und die Innovationsanstrengungen werden nur einmal unternommen ${ }^{2}$ ).

Der Einfluß des Wettbewerbsdrucks auf die Wahl des Innovationszeitpunkts läßt sich durch partielle Differentiation der Bedingung erster Ordnung nach $h$ ermitteln:

(D.17) $\operatorname{sign}\left[d T^{\star} / d h\right]=\operatorname{sign}\left[T^{\star}(r-g+h)-1\right]$,

d.h. $\quad \frac{d T^{\star}}{d h} \stackrel{\geq}{<}$.

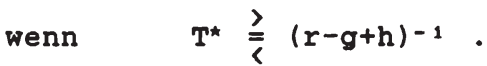

Eine Intensivierung des Wettbewerbs kann also sowohl zu einer Beschleunigung als auch zu einer Verzögerung der Innovationsaktivitäten führen. Eine Erklärung für diesen Vorzeichenwechsel ergibt sich aus folgender Oberlegung ${ }^{3}$ ).

Besteht keine Ronkurrenz, erreicht die Unternehmung ihren optimalen Innovationszeitpunkt, wenn sich die Grenznutzen und die Grenzkosten der Verschiebung des Innovationszeitpunkts ausgleichen'). Treten Konkurrenten auf den Plan, läuft das Unternehmen Gefahr, daß ihm andere mit der Innovation zuvorkommen und die Innovatorenrente abschöpfen. Der einsetzende Wettbewerb wird deshalb $z u$ einer Vorverlagerung des geplanten Innovationszeitpunkts führen, also $\mathrm{dT} / \mathrm{dh}<0$. steigt die Wettbewerbsintensität weiter an, vergrößern sich sowohl die Wahrscheinlichkeit, $d a \beta$ ein Ronkurrent vorzeitig innoviert, als auch die Rosten der Beschleunigung der eigenen Innovationsaktivitäten. Erreicht die Wahrscheinlichkeit der Innovati-

1) Das gleiche Ergebnis resultiert auch für $h=\left(P_{0}-P_{1}\right)=0$. Hier ist zwar Imitation möglich, sie bringt jedoch keinen Ertrag $\left(P_{2}=0\right)$.

2) Vgl. Kamien, M.I./Schwartz, N.L. (1972), S. $52 f$

3) Vgl. Kamien, M.I./Schwartz, N.L. (1976), S. $254 f$

4) Vgl. Abschnitt I.2 dieses Kapitels 
on durch einen Konkurrenten trotz forcierter eigener Entwicklungsaktivitäten einen kritischen Wert, ist es für das Unternehmen rational, den eigenen Innovationszeitpunkt zu verzögern und dadurch zwar auf die Quasi-Rente als Innovator zu verzichten, jedoch die Innovationskosten überproportional zu reduzieren.

Der Extremfall ist dann erreicht, wenn jeder Vorstoß des Unternehmens durch Konkurrentenreaktionen umgehend neutralisiert wird. Im vorliegenden Modell lautet die Marginalbedingung für $h=0, d . h$. alle Ronkurrenten agieren als Imitatoren:

$$
\frac{(r-g) P_{0}+k P_{1}}{r-g+k}=-C^{\prime}(T) \cdot e^{-(r-g) T} .
$$

Sofortige Ronkurrentenreaktion bedeutet, daß $\mathrm{k}$ unendlich gro $\beta$ wird mit der Konsequenz, daß die Quasi-Rente bei Imitation $P_{1}$ gegen null geht; die linke Seite von Gleichung (D.18) geht somit gegen null. Damit die Marginalbedingung erfüllt wird, muß auch die rechte seite der Gleichung (D.18) null werden. Dies ist bei der unterstellten Kostenfunktion dann der Fall, wenn $T$ gegen unendich geht. Damit ergibt sich das bekannte Ergebnis, daß bei vollkommenem Wettbewerb Innovationsanstrengungen unterbleiben, da Gewinne sofort wegkonkurriert werden ${ }^{1}$ ).

An dieser stelle soll kurz auf das in Abschnitt I.2 diese Kapitels bereits angesprochene Argument von Barzel2) eingegangen werden, daß Wettbewerb zu einer vorzeitigen Realisierung von Innovationsprojekten führt, was zwar nicht zu privaten, wohl aber zu gesellschaftlichen verlusten führt.

Barzel argumentiert in einem Modell, in dem nur der Innovator einen Gewinn erzielen kann. Zudem erfordert die Innovation fixe Kosten in Höhe von $C_{0}$.

Somit lautet der Ralkũl:

(D.19)

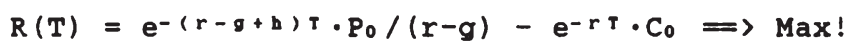

1) Vgl. Kamien, M.I./Schwartz, N.L. (1972), S.56

2) Vgl. Barzel, Y. (1968), S. $351 \mathrm{f}$ 
für die Bedingung erster ordnung ergibt sich:

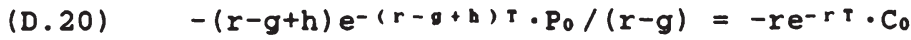

und somit

(D.21) $\quad T=(g-h)-1 \cdot \ln \left[r C_{0}(r-g) / P_{0}(r-g+h)\right]$.

Für die Bedingung zweiter ordnung muß gelten:

(D.22)

$$
(r-g+h)^{2} e^{-(r-g+b) T \cdot P_{0} /(r-g)}-r^{2} \cdot e^{-r T} C_{0}<0
$$

bzw. nach Einsetzen von Gleichung (D.20):

(D.23)

$$
(r-g+h) r e^{-r T} \cdot C_{0}<r^{2} e^{-r T} \cdot C_{0}
$$

und somit

(D.24) g>h.

Der optimale Innovationszeitpunkt soll nun verglichen werden mit dem Einführungszeitpunkt to, in dem die Innovation keinen Gewinn erbringt; also

(D.25)

$$
e^{-(r-g+b) t \cdot \cdot P_{0} /(r-g)}=e^{-r t \cdot \cdot C_{0}}
$$

somit

(D.26)

$$
t^{0}=(g-h)-1 \cdot \ln \left[C_{0}(r-g) / P_{0}\right] .
$$

Wegen Gleichung (D.24) zusammen mit (D.21) und (D.26) gilt immer:

(D.27) $\quad T>t^{0}$,

d.h. der Wettbewerb führt nicht zu einer so frühzeitigen Realisierung des Innovationsprojekts, daß die Gewinne völlig verschwinden')

1) Dabei liegt $T$ im Vergleich $z u$ to um so später, je stärker der Kostenanstieg bei einer Vorziehung des Innovationszeitpunkts ist. 
Der Einfluß der weiteren Modellvariablen auf den Innovationszeitpunkt läßt sich ebenfalls durch partielle Differentiation der Marginalbedingung ermitteln' ${ }^{1}$.

Die Differentiation nach $P_{0}$ und $P_{1}$ ergibt, daß eine Erhöhung der Innovationsgewinne eindeutig innovationsbeschleunigend wirkt:

(D.28)

$$
\frac{d R^{\prime}(T)}{d P_{0}}=-\frac{r-g+h}{r-g+k} \cdot e^{-(r-g+b) T}<0
$$

und

(D.29)

$$
\frac{d R^{\prime}(T)}{d P_{1}}=\left[\frac{r-g+h}{r-g+k}-\frac{r-g+h}{r-g}\right] \cdot e^{-(r-g+b) T}<0 .
$$

Eine Anderung der Imitationsgewinne führt nicht zu eindeutigen Wirkungen auf den Innovationszeitpunkt:

(D.30)

$$
\frac{d R^{\prime}(T)}{d P_{2}}=\frac{r-g+h}{r-g+k} \cdot e^{-(r-g+D) T}-e^{-(r-g) T} \stackrel{\geqslant}{=},
$$

je nachdem ob

$$
\text { (D.31) } \quad \mathrm{T} \leqq \ln \left[\frac{\mathrm{P}_{0}-\mathrm{P}_{1}}{\mathrm{r}-\mathrm{g}+\mathrm{k}}\right] .
$$

Je nach Marktstruktur führt eine Erhöhung der Imitationsgewinne zu einer Verzögerung oder einer Beschleunigung des geplanten Markteintritts. Eine Verschärfung des Imitationswettbewerbs führt immer zu einer Verschiebung des geplanten Markteintritts in die zukunft:

(D.32)

$$
\frac{d R^{\prime}(T)}{d k}=(r-g+h) \cdot e^{-(r-g+b) T} \cdot \frac{P_{0}-P_{1}}{(r-g+k)^{2}}>0 .
$$

In dem vorgestellten Modell wird ein erwartungswertmaximierender Innovationszeitplan aufgestellt. An ihm wird unbeeinflußt von den tatsāchlichen Ausprägungen des Konkurrentenver-

1) Vgl. Kamien, M.I./Schwartz, N.L. (1972), S. $56 f$ 
haltens festgehalten. Die Ausgabenstruktur entspricht somit einem Innovationsauftrag mit genauer Festlegung des Zeitpunkts der Leistungserbringung. Sind jedoch die Imitationsgewinne gering oder können keine erzielt werden, zum Beispiel bei Patentschutz für die Innovation oder bei Exklusiv-verträgen in Nachfragemonopolen (vgl. das Post- und Fernmeldewesen), wird das Unternehmen seine Forschungs- und Entwicklungsanstrengungen abbrechen und auf andere Projekte verlagern, wenn ein Ronkurrent innoviert.

Die Innovationsanstrengungen $Y(t)$ werden also nur solange fortgesetzt, wie noch kein Ronkurrent auf den Markt getreten ist. Im Falle der Verhinderung von Imitationswettbewerb beispielsweise durch Patentschutz lautet der Kalkül für den optimalen Zeit- Ausgabenplan:

$$
[1-F(T)] \int_{t=1}^{T+L} e^{-r t} \cdot P d t-\int_{t=0}^{T} e^{-r t} \cdot y(t)[1-F(t)] d t \Rightarrow \operatorname{Max} !
$$

Der erste summand gibt den Barwert der mit der Wahrscheinlichkeit [1 - F(T)] zu erzielenden Innovationsgewinne an, der zweite die geplanten Ausgaben, die beim Auftreten eines Konkurrenten gestoppt und deshalb mit der entsprechenden Wahrscheinlichkeit gewichtet werden.

Um den optimalen Innovationszeitplan aus Gleichung (D.33) zu berechnen, muß eine kumulierte Ausgabenfunktion $C(Y(t))$ zugrunde gelegt werden, für die beim Eintreten eines bestimmten Ausgabenniveaus der Erwartungswert der Lösung der Innovationsaufgabe gleich eins wird. Auf die Ableitung dieser Funktion soll hier verzichtet werden'). Nur auf die Ergebnisse sei eingegangen ${ }^{2}$ ). Der geplante Markteintritt erfolgt um so später, je geringer die Quasi-Rente $P$. und je höher die erwarteten kumulierten Rosten $C(Y(t))$ sind. Auch für diese Ronstellation läßt sich ein optimaler Wettbewerbsgrad bestimmen. Er liegt um so höher, je höher die Patentrente und je länger die Patentlaufzeit sind ${ }^{3}$ ).

1) Vgl. Kamien, M.I./Schwartz, N.L. (1974), S. 184ff

2) Vgl. Kamien, M.I./Schwartz, N.L. (1974), S. $186 f$ und dieselben (1976), S. $255 \mathrm{ff}$

3) Vgl. Kamien, M.I./Schwartz, N.L. (1976), S. 258 
In bezug auf den Patentschutz ist das Ergebnis interessant, daß zwar die Entwicklungsdauer um so kürzer ist, je länger der Patentschutz dauert. Es gibt jedoch Konstellationen, in denen trotz unbegrenzten Patentschutzes ein Projekt nicht unternommen wird. Dies ist dann der Fall, wenn der Erwartungswert der Aufwendungen aufgrund hoher Ronkurrenzintensität die Patentrente übersteigt. In solchen Fällen sichert nur die Gewährung eines Monopols die Realisierung des Projekts').

\section{$1.2 \quad$ Zusammenfassung}

Die beiden hier vorgestellten Varianten eines entscheidungstheoretischen Modells des Innovationswettbewerbs führen zu übereinstimmenden Ergebnissen. Sowohl bei Annahme eines exante bestimmten und später unbeeinflußt vom tatsächlichen Konkurrentenverhalten beibehaltenen Forschungsplans als auch bei der Unterstellung, daß die Innovationsanstrengungen nach dem Markteintritt eines Konkurrenten gestoppt werden, fūhrt eine Erhöhung der Innovations-bzw. Patentrente zu einer Forcierung der Innovationsbemühungen. Da die Unternehmen Erwartungswerte maximieren, die durch die Konkurrenzintensität bestimmt werden, gibt es in beiden Modellen einen optimalen Wettbewerbsgrad. Optimalitäskriterium ist dabei die Minimierung des Innovationszeitraums. Die optimale Wettbewerbsintensität liegt um so höher, je größer die Innovationsgewinne sind, d.h. je höher die Quasi-Renten und je geringer die Rosten sind.

Der vorgestellte entscheidungstheoretische Ansatz dient der Ermittlung des optimalen Innovationsausgabenplans. Unter diesem Aspekt ist die Analyse dynamisch. Allerdings wird das Konkurrentenverhalten nur über subjektive Wahrscheinlichkeitsverteilungen berücksichtigt. Das Ronkurrentenverhalten wird folglich als gegeben vorausgesetzt ${ }^{2}$. . Dieser Mangel soll im folgenden spieltheoretischen Ansatz durch die Endogenisierung der Konkurrentenreaktionen behoben werden.

1) Vgl. Kamien, M.I./Schwartz, N.L. (1974), S. 186

2) Vgl. Reinganum, J.F. (1981), S. 22 
Die Fragestellung des folgenden Modells') entspricht der zweiten Variante des zuvor besprochenen entscheidungstheoretischen Ansatzes. In einem Patentrennen konkurrieren $n$ Unternehmen um die Patentrente $V$. Das Unternehmen, das als erstes die Innovation auf den Markt bringt, erhält das Patent. Für jeden der $n$ Konkurrenten beträgt die Wahrscheinlichkeit, daß die Forschungsanstrengungen im Zeitpunkt $T$ erfolgreich abgeschlossen werden können:

$$
F(T)=1-e^{-b(x) t} ;
$$

Gleichung (D.34) beschreibt die technologische Unsicherheit. Der Erwartungswert für den Innovationszeitpunkt beträgt:

$$
\operatorname{ET}(x)=h(x)-1
$$

Durch die Wahl ihrer Forschungs- und Entwicklungsausgaben $x$ können die Unternehmen den Erwartungswert ihres Innovationszeitpunkts beeinflussen.

Für $h(x)$ wird angenommen, $d a \beta h^{\prime}(x)>0$ und $h^{\prime \prime}(x)<0$. Es wird also eine klassische produktionsfunktion mit abnehmenden Grenzerträgen unterstell $t^{2}$ ).

Die Marktunsicherheit für ein Unternehmen $i$ besteht aufgrund der Unkenntnis der Innovationspläne seiner Konkurrenten $j \neq i$. Wie im vorigen Abschnitt sei

$$
v=\min _{j \neq 1}\{T(x,)\}
$$

Die Wahrscheinlichkeit, daß bis zum Zeitpunkt $t$ keiner der Ronkurrenten als Innovator auftritt, beträgt:

1) Zu diesem Modell vgl. Loury, G.C. (1979), S. 395ff; Lee, T./Wilde, L.L. (1980), S. $429 \mathrm{ff}$ sowie den grundlegenden Aufsatz von Scherer, F.M. (1967), S. $259 f$.

2) Loury, G.C. (1979), S. 398 und Lee, T./Wilde, L.L. (1980). S. 430 nehmen für einen Anfangsbereich steigende Skalenertrăge an: $h^{\prime \prime}(x)>0$ für $x$ $<x^{\prime}$. 
(D.37)

$$
W R(v \leq t)=1-e^{-\operatorname{tgh}(x)}
$$

bzw. mit $a=\sum_{j \neq 1} h\left(x_{j}\right)$

$$
W R(v s t)=1-e^{-t} \text {. }
$$

Die erwarteten Erträge aus dem Innovationsprojekt betragen dann:

$$
\begin{aligned}
E V & =\int_{t=0}^{\infty} W R(v=t)\left[\int_{s=0}^{t} W R(T=s) V e^{-r s} d s\right] d t \\
& =\int_{t=0}^{\infty} a e^{-a t} \cdot\left[\int_{s=0}^{t} h(x) e^{-h(x) s}\left(V e^{-r s}\right) d s\right] d t \\
& =\frac{h(x) \cdot V}{a+h(x)+r} .
\end{aligned}
$$

Die Kosten, fixe Rosten $F$ im Zeitpunkt $t=0$ sowie die laufenden Kosten $x$, werden nur solange eingegangen, bis einer der Konkurrenten $j$ oder das betrachtete Unternehmen $i$ selbst innovieren. Da das Unternehmen, das als erstes auf den Markt kommt, das Patent und somit die gesamte Quasi-Rente aus der Innovation erhält, wird dieses spezielle Projekt dann von den anderen Unternehmen nicht mehr weiter verfolgt.

Die erwarteten Rosten betragen somit:

(D. 39)

$$
\begin{aligned}
E C & =\int_{t=0}^{\infty}\left[\int_{s=0}^{t} x e^{-r s} d s\right] \cdot W R(v=t v T=t) d t+F \\
& =\int_{t=0}^{\infty}\left[\int_{s=0}^{t} x e^{-r s} d s\right] \cdot W R(a+h(x)) e^{-(a+h(x)) t} d t+F \\
& =\frac{x}{a+h(x)+r}+F .
\end{aligned}
$$

Der erwartete Gewinn beläuft sich nun auf:

$(D .40) \quad E R=E V-E C$

$$
=\frac{h(x) \cdot V-x}{a+h(x)+r}-F .
$$


Aus der Marginalbedingung für die Maximierung des erwarteten Gewinns

$$
\text { (D. 41) } \quad \frac{d E R}{d x}=\frac{(a+r)\left(h^{\prime}(x) \cdot v-1\right)-\left[h(x)-x h^{\prime}(x)\right]}{(a+h(x)+r)^{2}}=0
$$

ergibt sich:

$$
\text { (D. 42) } \quad V=\frac{a+h(x)+r-h^{\prime}(x)}{(a+r) h^{\prime}(x)}
$$

und somit für den erwarteten Gewinn bei optimalen variablen Ausgaben 8 :

$$
\text { (D. 43) } \quad E R=\frac{h(x)-s h^{\prime}(\hat{x})}{(a+r) h^{\prime}(\hat{x})}-F \text {. }
$$

Da das Unternehmen das Innovationsprojekt nur bei positiven Gewinnerwartungen realisieren wird, muß gelten:

$$
\text { (D.44) } \frac{h(\hat{x})-8 h^{\prime}(\hat{x})}{(a+r) h^{\prime}(\hat{x})}>F
$$

oder zumindest

$$
h(\hat{x})>8 h^{\prime}(\hat{x})
$$

Um den Einfluß einer Veränderung von a auf die geplanten optimalen Ausgaben $\&$ zu ermitteln, wird Gleichung (D.42) nach \& aufgelōst und total differenziert. Nach geeigneten Umformungen resultiert:

(D. 46) $\quad \frac{d s}{d a}=\frac{h^{\prime}(8) v-1}{[(a+r) v+s] \cdot h^{\prime \prime}(8)}$.

Wegen $h^{\prime \prime}(x)<0$ ist der Nenner negativ. Aus Gleichung (D.41) ist ersichtlich, daß der Wert des zählers von Gleichung (D.46) das gleiche vorzeichen hat wie die beiden summanden des zählers von Gleichung (D.41). Somit ist aufgrund von (D.45) der Zähler von Gleichung (D.46) positiv und de/da>0. Wird a als der Grad der Wettbewerbsintensität interpretiert, so impliziert Gleichung (D.46), daß eine Erhöhung des Wettbe- 
werbsdrucks $z u$ verstärkten Innovationsanstrengungen führt' '.

Die bisherigen Ableitungen beziehen sich auf das individuelle Unternehmensgleichgewicht. Für den Gesamtmarkt soll nun ein symmetrisches Cournot-Nash-Gleichgewicht ermittelt werden'2).

Für jedes der $n$ gleichen Unternehmen beträgt a nun

(D.47) $\quad a=(n-1) h(\hat{x})$.

Wird \& als Funktion von a definiert, also $\&=\mathrm{H}(\mathrm{a})$, ergibt sich aus dem totalen Differential unter Berücksichtigung von Gleichung (D.47):

(D. 48$)$

$$
\frac{d \hat{x}}{d n}=\frac{d H / d a \cdot h(\hat{8})}{1-(n-1) \cdot d H / d a \cdot h^{\prime}(\hat{x})} .
$$

Da $\mathrm{dH} / \mathrm{da}=\mathrm{d} R / \mathrm{da}>0$ ist, wird das Vorzeichen von Gleichung (D.48) durch den Nenner bestimmt.

Wegen $\mathrm{dH} / \mathrm{da}=\mathrm{d} \boldsymbol{d} / \mathrm{da}$ ist

$$
1-(n-1) \cdot \frac{d H}{d a} \cdot h^{\prime}(\hat{x})=\frac{[(a+r) v+\hat{x}] h^{\prime \prime}(x)+\left[h^{\prime}(\hat{x}) v-1\right](n-1)}{[(a+r) v+\hat{x}] h^{\prime \prime}(\hat{x})} ;
$$

aus der Bedingung zweiter Ordnung mit $a=(n-1) h(\hat{x})$

(D. 50)

$$
\frac{d E R^{2}}{d^{2} x}=\frac{[(a+r) v+x] h^{\prime \prime}(x)+\left[h^{\prime}(x) v-1\right](n-1) h^{\prime}(x)}{a+h(x)+r}
$$

folgt :

(D.51) $\mathrm{d} 8 / \mathrm{dn}>0$.

1) Dieses Ergebnis ist von der unterstellten Kostenfunktion abhăngig. Loury, G.C. (1979), nimmt nur fixe Kosten an und kommt zum unter diesen Annahmen plausiblen gegenteiligen Ergebnis (S. 400). Zur Interpretation dieser Differenzen vgl. Reinganum, J.F. (1982), S. $683 f$ und dieselbe (1984), S. 61ff.

2) $\mathrm{Zu}$ anderen Gleichgewichtskonzepten vgl. Scherer, F.M. (1967), S. $373 f$ 
D.h. mit wachsender Anzahl von Unternehmen steigt das Ausmaß der Innovationsanstrengungen auf dem Markt.

Mit Hilfe dieses Ergebnisses läßt sich jetzt die Abhängigkeit des erwarteten Innovationszeitpunkts von der Anzahl der Marktteilnehmer bestimmen.

Der Innovationszeitpunkt auf dem Markt sei definiert durch:

(D.52) $\quad T(n)=\min _{0 \leq 1 \leq \pm} T\left(R_{1}\right) ;$

der im Gleichgewicht erwartete Wert von $T(n)$ beträgt dann:

$$
T(n)=(n \cdot h(x))-1
$$

Eine Erhöhung der Anzahl der Marktteilnehmer führt zu einer Verkürzung des Innovationszeitraums auf dem betreffenden Markt, wenn gilt:

$$
\text { (D.54) } \frac{\operatorname{dET}(n)}{\operatorname{dn}}<0 \text { bzw. (D.55) } \frac{\mathrm{d}[1 / \mathrm{ET}(\mathrm{n})]}{\mathrm{dn}}>0 \text {. }
$$

Diese Bedingung ist erfüllt, da

$$
\text { (D.56) } \frac{d[1 / E T(n)]}{d n}=h(s(n))+n h^{\prime}(x(n)) \cdot \frac{d s}{d n}>0
$$

wegen $n, h(x), h^{\prime}(x)$ und $d x / d n>0$.

Somit führt der Markteintritt zusätzlicher potentieller Innovatoren $z u$ einer Beschleunigung der Innovationsanstrengungen und zu einer Verkürzung der Innovationsdauer. Der Marktzutritt hält so lange an, wie Gewinne erzielt werden können, also

$$
E R>0
$$

bzw. aus Gleichungen (D.40) und (D.41):

(D.57) $h^{\prime}(8)(V-F)>1$.

Der Marktzutritt weiterer Wettbewerber endet um so früher, je höher die fixen Kosten $F$, je geringer die Patentrente $V$ und 
um so höher das optimale Ausgabenniveau $\hat{x}^{1}$, bzw. wegen $\mathrm{d} \mathbf{x} / \mathrm{dn}$ > 0 je größer die Anzahl der bereits im Markt vertretenen Unternehmen ist. Wie im entscheidungstheoretischen Modell sind auch hier die Gewinnmöglichkeiten die wichtigste Determinante der Innovationsbereitschaft. Zusätzlich wird deutlich, daß fixe Rosten ceteribus paribus innovationshemmend wirken.

\section{$\underline{2.2 \quad Z u s a m m e n f a s s u n g}$}

In dem dargestellten spieltheoretischen Modell wird die optimale strategie eines potentiellen Innovators durch ein Cournot-Nash-Gleichgewicht bestimmt. Für die Innovationsausgaben wird unterstellt, daß zu Beginn der Aktivitäten fixe Rosten anfallen und in jeder Periode so lange weiter investiert wird, bis eines der konkurrierenden Unternehmen als Innovator auf den Markt tritt. Je höher der Wettbewerbsdruck auf die potentiellen Innovatoren ist, desto stärker werden sie ihre Innovationsanstrengungen intensivieren. Entsprechend steigen mit der Anzahl der Ronkurrenten auch die gesamten auf dem Markt getätigten Innovationsausgaben. Da der Innovationserfolg um so wahrscheinlicher ist, je höher die Innovationsanstrengungen sind, sinkt mit steigender Anzahl der Ronkurrenten der Erwartungswert des Innovationszeitpunkts, d.h. der erwartete Innovationserfolg liegt zeitlich um so früher, je höher die Anzahl der Wettbewerber ist.

Die Bestimmung der optimalen Innovationsstrategien erfolgt unter Einbeziehung der optimalen Konkurrentenreaktionen auf das eigene Handeln. In diesem Sinn ist das Modell dynamisch. Allerdings wird hier nicht wie im entscheidungstheoretischen Ansatz ein optimaler Ausgabenplan ermittelt. Unter diesem Aspekt ist das spieltheoretische Modell statisch, d.h. die geplanten Ausgaben sind in allen Perioden gleich ${ }^{2}$ ).

1) Bei abnehmenden Grenzertrăgen ist dann $h^{\prime}(x)$ um so kleiner.

2) Vgl. Reinganum, J.F. (1981), S. 22 
Die beiden vorgestellten Ansätze sind in mehreren Richtungen unvollständig. Da die für die Innovationspolitik bedeutsamen, grundsätzlichen Ergebnisse bestehen bleiben, wird über die verschiedenen Modellerweiterungen nur ein Oberblick gegeben. während im entscheidungstheoretischen Ansatz das Ronkurrentenverhalten als gegeben unterstellt wurde, wurde für das spieltheoretische Modell eine konstante R\&D-Investition pro Periode angenommen und damit der Aspekt der optimalen Ausgabenplanung vernachlässigt. Durch Integration der beiden Ansätze in einem kontrolltheoretischen Modell können diese Mängel beseitigt werden. Dabei wird das Ergebnis des spieltheoretischen Ansatzes bestätigt, wonach bei wirksamem Patentschutz eine Erhöhung der Anzahl der Ronkurrenten zu einer Beschleunigung der Innovationstätigkeit führt'). Je unvollkommener jedoch der Patentschutz und je intensiver der Imitationswettbewerb, d.h. je stärker die Innovatorenrente im Imitationswettbewerb aufgezehrt wird, desto eher wird eine Position des Abwartens lohnend. Bei vollkommenem Wettbewerb kommt dann die Innovationstätigkeit zum Erliegen²).

Die bisherige Analyse bezieht sich isoliert auf ein bestimmtes Innovationsprojekt. Der Innovationsproze $\beta$ im sinne Schumpeters ist jedoch mehrstufig. Jedes abgeschlossene Innovationsprojekt ist nur eine $\mathrm{Zwischenstufe}$ im Innovationswettbewerb $\left.^{3}\right)$. In einem solchen sequentiellen Proze $\beta$ ist der bisher betrachtete Innovationswettbewerb jeweils eine stufe des gesamten spiels. Zwei Arten von Konkurrenten sind zu unterscheiden. Zum einen Unternehmen, die aufgrund einer Innovation ein Patent besitzen und auf dem Markt etabliert sind. Zum anderen Herausforderer, die in einem Proze $\beta$ schöpferischer zerstörung die Etablierten durch Verbesserungsinnovationen verdrängen wollen.

Wie im einstufigen Modell steigen mit der Anzahl der Wettbewerber und der Höhe der Innovationserträge die Innovationsan-

1) Vgl. Reinganum, J.F. (1982), S. 684

2) Vgl. Reinganum, J.F. (1982), S. 685ff; vgl. auch die Ergebnisse im entscheidungstheoretischen Ansatz.

3) Zu diesen Modellansătzen vgl. Friedman, J.H. (1986), S. 71ff 
strengungen ${ }^{1}$. Die strategie der Herausforderer und des jeweils etablierten Unternehmens weichen allerdings voneinander ab. Der Etablierte investiert auf jeder stufe des Prozesses weniger in neue Projekte als seine Herausforderer'). Der Grund dafür ist, $d a \beta$ er zwar durch die Investitionen die Wahrscheinlichkeit dafür erhöhen kann, daß er auf der nächsten stufe wieder das Patent erhält, er dadurch aber gleichzeitig die erwartete Nutzungszeit seines laufenden Patents verkürzt. Je attraktiver seine aktuelle Position desto geringer werden seine Innovationsbemühungen sein ${ }^{3}$ ). Analog sind auf der jeweiligen stufe die Innovationsanstrengungen um so geringer, je günstiger die zukünftigen Innovationsmöglichkeiten eingeschätzt werden').

\section{WOHLFAHRTSWIRRUNGEN}

Bei der Beurteilung der Wohlfahrtseffekte des Innovationswettbewerbs sind zwei gegenläufige Wirkungsrichtungen $z u$ beachten.

Die im Innovationsprozeß aktiven Unternehmen beziehen in ihren Kalkül auch die Aktivitäten der Konkurrenten mit ein. Allerdings beschränkt sich diese Einbeziehung der Konkurrenzbeziehungen auf die Wahrscheinlichkeit des Ronkurrenteneintritts bei der Bildung des Erwartungswerts der Quasi-Rente, die im Falle einer erfolgreichen Innovation abgeschöpft werden kann. Was die Unternehmen nicht berücksichtigen, ist der Verlust an erwartetem Ertrag, der auftritt, wenn der Innovationsprozeß durch die erfolgreiche Innovation eines Unternehmens beendet wird. Durch den Innovationserfolg entstehen bei den unterlegenen Unternehmen externe Rosten, die bei der Bestimmung der Innovationsstrategie nicht berücksichtigt wurden's). Dies führt zu einem Innovationstempo, welches über das

1) Vgl. Reinganum, J.F. (1985), S. 93

2) Vgl. Reinganum, J.F. (1985), S. 89; ähnlich auch Katz, M.L./Shapiro, C. (1987), S. $406 \mathrm{ff}$

3) Vgl. Reinganum, J.F. (1983), S. $745 f$

4) Vgl. Reinganum, J.F. (1985), S. $94 f$

5) Mit diesem Argument wird unterstellt, daß die unterlegenen Unternehmen aus den bis zum Innovationszeitpunkt des Konkurenten gemachten Investitionen keine Ertrăge erzielen können. 
soziale Optimum hinausgeht').

Dieser überoptimalen Innovationstätigkeit steht aber entgegen, daß sich die Unternehmen die Erträge ihrer Innovationsanstrengungen nur teilweise aneignen können, d.h. daß die Forschungs- und Entwicklungsergebnisse zumindest partiell die Eigenschaft öffentlicher Güter besitzen. Kann sich das Unternehmen jedoch nur Teile seiner Innovationserträge aneignen, reduziert sich sein erwarteter Gewinn. Das Unternehmen wird deshalb seine Innovationsanstrengungen im Vergleich zum gesellschaftlichen optimum reduzieren. Theoretisch könnten die mangelnden privaten Anreize durch Subventionen korrigiert werden'). Praktisch scheitert dieser vorschlag an den für seine Realisierung erforderlichen Informationserfordernissen. Auch können die mit einer solchen strategie der Internalisierung externer Effekte verbundenen administrativen Kosten zu negativen Anreizen für die Innovationstătigkeit der Unternehmen führen ${ }^{3}$ ).

Gleichzeitig ist zu beachten, daß die Forschungsergebnisse, die den charakter eines öffentlichen Gutes haben, von den Ronkurrenten verwertet werden können. Soweit dies möglich ist, können sie dann auf Parallelforschung verzichten'). Je höher der öffentliche-Guts-Anteil an der Forschungs- und Entwicklungstätigkeit ist, um so stärker werden die eigenen Innovationsanstrengungen reduziert. Der Gesamteffekt in bezug auf das Pareto-optimum ist für jede einzelne Innovation von der Ertragsstruktur, der stäke der Externalitäten und ihrer Verwendbarkeit bei Dritten abhängig's).

Aus diesen Ausführungen ergibt sich, daß ein Pareto-optimum aufgrund der Externalitäten und ihrer nur teilweisen Kompensation durch das Patentsystem nur zufälig erreicht werden kann. Die Richtung der Abweichung ist nicht eindeutig und nur für den Einzelfall bestimmbar. Läßt sich auch die Richtung der Abweichung vom Pareto-optimum nicht generell bestimmen,

1) Vgl. Mortensen, D.T. (1981), S. 969ff; Loury, G.C. (1979), S. 405f; Lee, T./Wilde, L.L. (1980), S. $434 f$

2) Vgl. Spence, A.M. (1984), S. $103 \mathrm{ff}$

3) Vgl. Capitelli, R./Müller, H.H. (1986), S.547ff

4) Vgl. Spence, A.M. (1984), S. 107

5) Vgl. Reinganum, J.F. (1981), S. $36 \mathrm{ff}$ 
so kann doch eine Aussage zum Wohlfahrtseffekt der Innovationstätigkeit gemacht werden. Wird durch eine Innovation ein neuer Markt erschlossen, so wirkt eine Innovation immer wohlfahrtssteigernd. Da die Unternehmen in ihre Innovationsplanung die Konkurrentenaktivitäten einbeziehen, werden die geplanten Kosten den erwarteten Innovationsgewinn nicht übersteigen. Zusätzlich erhalten die Konsumenten einen Rentenzuwachs' ${ }^{1}$.

IV. ZUSAMMENFASSUNG

In diesem Rapitel wurde untersucht, wie der von einem Unternehmen geplante Innovationszeitpunkt durch Konkurrenzbeziehungen beeinflußt wird. Während im spieltheoretischen Modell und seinen Erweiterungen eine Intensivierung des Wettbewerbs, d.h. eine Erhöhung der Anzahl der innovationsaktiven Unternehmen, immer eine Beschleunigung des Innovationsprozesses bewirkt, führt im entscheidungstheoretischen Ansatz verstärkter Wettbewerb zwar zunächst auch zu einer Erhöhung des Innovationstempos. Obersteigt die Wettbewerbsintensität jedoch einen kritischen Grad, so wird der Innovationszeitpunkt verzögert, da aufgrund der Imitationsgewinne eine abwartende Position lohnend wird.

Oberlegungen $z u$ den Wohlfahrtseffekten der Innovationstätigkeit ergeben, daß zwar durch ein generell gültiges Patentsystem die in jedem Einzelfall verschiedenen Externalitäten nur unvollkommen kompensiert werden können und deshalb ein Pareto-Optimum nur zufällig erreicht werden wird, daß aber die Realisierung von Innovationen immer wohlfahrtssteigernd ist.

Im folgenden Teil der Arbeit werden die Wirkungen von Steuern und Finanzierungsbedingungen auf die Risiko- und Innovationsbereitschaft untersucht.

1) Vgl. Usher, D. (1964), S. $282 f$ sowie Scherer, F.M. (1967), S. $392 f$. Führt die Innovation $z u$ einer Unverteilung der Nachfrage auf einem Markt mit konstantem Volumen, kann kein eindeutiger Wohlfahrtseffekt abgeleitet werden. Ubersteigen die zusätzlich möglichen Gewinne durch Kostensenkungen und erhöhten Ronsumentenrente die Innovationskosten, dann ist auch hier ein positiver Wohlfahrtseffekt festzustellen; vgl. Scherer, F.M. (1967), S. 293. 
TEIL 2: DER EINFLUSS VON STEUERN UND FINANZIERUNGSBEDINGUNGEN BEDINGUNGEN AUF DIE RISIRO- UND INNOVATIONSBEREITBEREITSCHAFT

E. DER EINFLUSS DER BESTEUERUNG AUF DIE BEREITSCHAFT ZUR RISIROOBERNAHME

Wie in Kapitel $C$ herausgearbeitet wurde, ist für Innovationen ein im Vergleich zu anderen Arten von Investitionen erhöhtes Risiko typisch. Im folgenden sollen die wirkungen der Besteuerung auf die Risikobereitschaft untersucht werden.

Diese Problemstellung wird zunächst innerhalb eines Modells der Portefeuille-Bildung analysiert. Untersucht wird, wie sich die Besteuerung auf die struktur der im Portefeuille gehaltenen Titel auswirkt. Anschließend wird die Analyse erweitert und gefragt, wie die Besteuerung oder die Anderung steuerlicher Institutionen auf die Entscheidung über Investitionsobjekte wirkt, die vor der Besteuerung als gleichwertig erachtet werden. Während im Portefeuille-Modell die Wirkungen der Besteuerung auf die Bereitschaft zur Obernahme von Risiken aus der sicht des Kapitalanlegers diskutiert werden, entspricht der zweite Ansatz dem Entscheidungsproblem eines (potentiellen) Innovators, der dem erwarteten Nutzen aus einem risikobehafteten Innovationsvorhaben die Nutzen gegenüberstellt, die er aus einem alternativen, risikolosen Engagement ziehen kann.

Aufbauend auf diesen Ansätzen werden dann einzelne steuerliche Regelungen, welche die Investitions- und insbesondere die Innovationsbereitschaft betreffen, untersucht. 


\section{1.} Die Modellgrundlagen

In diesem Modell soll die Frage beantwortet werden, wie ein gegebenes Anfangsvermögen auf risikobehaftete und sichere Anlageformen aufzuteilen ist, damit der erwartete Nutzen aus der Vermögensanlage des Entscheidungsträgers maximiert wird. Das verwendete Erwartungsnutzenkonzept' ${ }^{\prime}$ läßt allgemeinere Schlußfolgerungen $z u$ als der in der früheren Literatur übliche Mittelwert-Varianz-Ansatz'), da die unterstellte Nutzenfunktion keinen Beschrănkungen unterliegt.

Die zielfunktion des Individuums für den Erwartungsnutzen des Endvermögens $\$$ lautet ${ }^{3}$ ) :

$$
E[U(W)] \Longrightarrow \operatorname{Max} !
$$

Das Anfangsvermögen Wo wird auf die riskante Anlage mit der Ertragsrate $x$ und die sichere Anlageform mit der Verzinsung $r$ aufgeteilt. Der Anteil der riskanten Anlage im Portefeuille beträgt a. Das Endvermögen (W) beträgt:

$$
W=[1+r+a(X-r)] \cdot W_{0} .
$$

Sein Nutzenmaximum erreicht der Investor dann, wenn er für seine Grenzinvestition die folgende (Marginal-)Bedingung erfüllt:

1) Dieses Konzept wurde zuerst verwendet von stiglitz, J.E. (1969), S. $263 f f$ und Mossin, J. (1968), S. 74ff; vgl. auch Ahsan, S.M. (1974), S. 318ff; Haegert, L./Kramm, R. (1975), S. 69ff; Sandmo, A. (1985), S. 293ff; Cansier, D. (1985), S. $403 f f$ und Swoboda, P./Zechner, J. (1985), S. $403 f f$.

2) Vgl. Tobin, J. (1958), S. 65ff; Richter, M.K. (1960), S. 152ff; Penner, R.G. (1964), S. $83 f f$ sowie mit einem Erwartungswert-Verlust-Ansatz Domar, E.D./Musgrave, R.A. (1944), S. 388ff; ein Uberblick über die verschiedenen Ansätze findet sich bei Allingham, M.G. (1972), S. $205 f \mathrm{f}$.

3) Zur Vorgehensweise vgl. Atkinson, A.B./Stiglitz, J.E. (1980), S. 104ff 
(E. 3)

$$
E\left[U^{\prime}(W) \cdot(X-r)\right]=0
$$

bzw.

$$
E\left[U^{\prime}(W) \cdot X\right]=U^{\prime}(W) \cdot r \text {. }
$$

D.h. für ein Nutzenmaximum hat der Investor sein Vermögen so aufzuteilen, daß sich die Grenznutzen aus den verschiedenen Anlageformen ausgleichen.

Bei Einführung einer proportionalen Ertragsteuer mit dem steuersatz $\left.\tau(0<\tau<1)^{1}\right)$ ändern sich die Gleichungen (E.2) und (E. 3) zu

(E.4) $W_{r}=[1+(1-\tau)\{r+a(X-r)\}] \cdot W_{0}$

mit $W_{r}$ : Vermögensendwert nach steuern,

und

(E.5) $\quad(1-\tau) E\left[U^{\prime}\left(W_{\tau}\right)(X-r)\right]=0$.

Da $(1-\tau) \neq 0$, kann Gleichung (E.5) durch diesen Ausdruck dividiert werden:

$$
E\left[U^{\prime}\left(W_{r}\right)(X-r)\right]=0 \text {. }
$$

Bei unterschiedsloser Besteuerung der Erträge verschiedener Rapitalanlagen ändert sich die Optimalbedingung offensichtlich nicht. Um die steuerwirkungen auf die Bereitschaft zur Risikoübernahme festzustellen, ist abzuleiten, wie sich der Anteil der risikobehafteten Investitionen im Portefeuille mit der Besteuerung verändert. Implizite Differentiation von Gleichung (E.6) ergibt:

(E.7)

$$
\begin{aligned}
\frac{d a}{d \tau} & =\frac{E\left\{U^{\prime \prime}\left(W_{r}\right)(X-r)[r+a(X-r)]\right\}}{(1-\tau) E\left\{U^{\prime \prime}\left(W_{\tau}\right)(X-r)^{2}\right\}} \\
& =\frac{a}{1-\tau}+\frac{r}{1-\tau} \cdot \frac{E\left\{U^{\prime \prime}\left(W_{\tau}\right)(X-r)\right\}}{E\left\{U^{\prime \prime}\left(W_{\tau}\right)(X-r)^{2}\right\}} .
\end{aligned}
$$

1) Die Ertragsraten $r$ und $X$ unterliegen keinen Restriktionen. Es wird ein sofortiger Verlustausgleich mit dem steuersatz $\tau$ unterstellt. 
Zusätzlich wird Gleichung (E.6) implizit differenziert, mit $A=a \cdot W_{0}:$

(E.8) $\frac{\mathrm{dA}}{\mathrm{d} W_{0}}=-\frac{1+r(1-\tau)}{1-\tau} \cdot \frac{\mathrm{E}\left\{U^{\prime \prime}\left(W_{\tau}\right)(\mathrm{X}-r)\right\}}{\mathrm{E}\left\{U^{\prime \prime}\left(W_{r}\right)(X-r)^{2}\right\}}$

und in (E.7) eingesetzt:

(E.9) $\frac{d a}{d \tau}=\frac{a}{1-\tau}-\frac{r}{1+r(1-\tau)} \cdot \frac{d A}{d W_{0}}$.

Wird der zweite Term auf der rechten seite von Gleichung (E.9) mit Wo erweitert und die Gleichung entsprechend umgestellt, resultieren die steuerwirkungen auf die Aufteilung des Portefeuilles in Abhängigkeit von der Vermögenselastizitāt der Nachfrage nach riskanten Titeln (dA/A : dWo/Wo):

$$
\frac{d a}{d \tau}=\frac{a}{1-\tau} \cdot\left[1-\frac{r(1-\tau)}{1+r(1-\tau)} \cdot\left(\frac{d A}{A}: \frac{d W_{0}}{W_{0}}\right)\right] .
$$

\section{$\underline{2 .}$} Die sichere Anlageform erbringt keinen Ertrag $(r=0)$

Erbringt der risikolose Titel keinen Ertrag, beispielsweise bei Anlage in flüssigen Mitteln im Falle von Preisniveaustabilität, dann reduziert sich Gleichung (E.10) zu:

$$
\frac{d a}{d \tau}=\frac{a}{1-\tau}
$$

Die Wirkung der Erhebung einer proportionalen Einkommensteuer auf die Risikobereitschaft ist eindeutig. Der riskant investierte Vermögensanteil wird auf das $1 /(1-\tau)$-fache erhöht. Dieses Investorenverhalten kann mit folgender uberlegung erklärt werden').

Vor steuern ist der Erwartungsnutzen dann maximal, wenn der Vermögensanteil a riskant angelegt wird. Da die Grenznutzen der Geldanlage annahmegemäß nicht negativ sind, sinkt bei Besteuerung der Erwartungsnutzen auf:

1) Zum Folgenden vgl. Mossin, J. (1968), S. $75 \mathrm{ff}$ 
(E.12) $\quad E\left[U\left(W_{0}\{1+(1-\tau)\right.\right.$ aX $\left.\left.\}\right)\right]$.

Das maximale Erwartungsnutzenniveau kann jedoch durch Vermögensumschichtung genau dann wieder erreicht werden, wenn der nach steuern risikobehaftet angelegte Vermögensanteil ar die folgende Bedingung erfüllt:

(E.13)

$$
a_{r} \cdot(1-\tau)=a \text {. }
$$

Durch diese Umschichtung bleibt das erwartete Endvermögen vor und nach steuern gleich').

Bestehen keine Verschuldungsmöglichkeiten, so kann das alte Nutzenniveau nur dann wieder erreicht werden, wenn der vor steuern riskant investierte Vermögensanteil nicht größer als $(1-\tau)$ ist.

Abschließend soll die eben analysierte Problemstellung graphisch erläutert werden').

Abbildung B.1: Portefeuille-struktur mit $r=0$

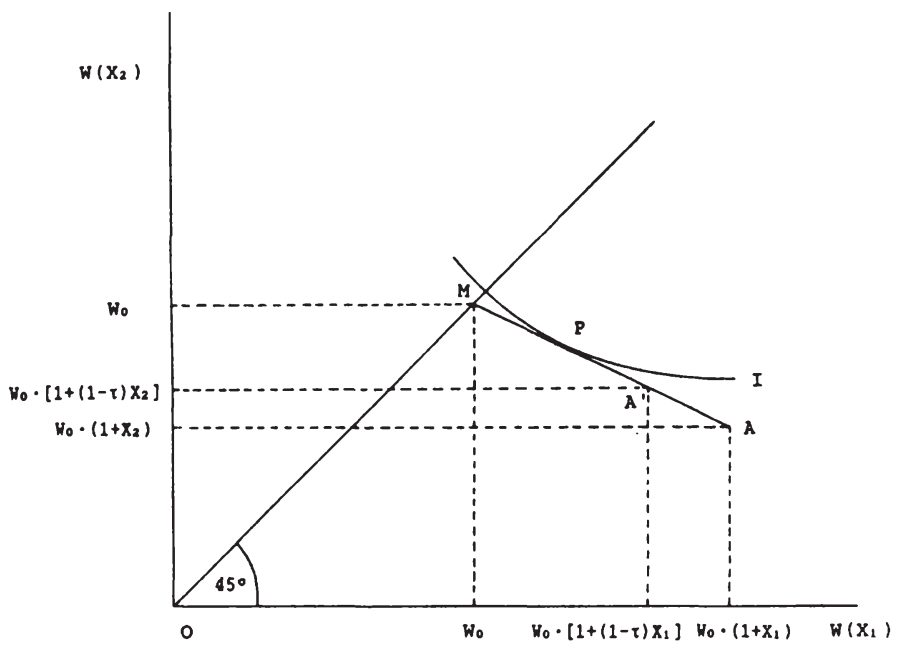

1) Vgl. Sandmo, A. (1985), S. 295

2) Vgl. Stiglitz, J.E. (1969), S. 273 
Werden zwei mögliche Realisierungen für $\mathrm{X}$ mit $\mathrm{X}_{1}>0$ und $\mathrm{X}_{2}<0$ zugelassen, repräsentiert die strecke MA die Budgetlinie vor steuern. In $M$ (A) wird das gesamte Vermögen sicher (riskant) angelegt. Der Erwartungsnutzen wird im Punkt $P$ maximiert. $\mathrm{Da}$ die sichere Anlage ertraglos ist und somit auch keine steuer anfält, beginnt die Budgetlinie nach steuern weiterhin in $M$. Ihre steigung bleibt unverändert, da Gewinne und Verluste symmetrisch behandelt werden. Aufgrund der Besteuerung der Erträge aus der risikobehafteten Anlage endet die Budgetlinie nun in $A^{\prime}$. Der Erwartungswer.t wird weiterhin in $\mathbf{P}$ maximiert. Der Anteil der riskanten Anlageformen im Portefeuille ist jedoch als Folge des substitutionseffekts von MP/MA auf MP/MA' gestiegen') .

3. Die sichere Anlageform erbringt Ertrag $(r>0)$

Ist auch die sichere Vermögensanlage ertragbringend, gilt für die Anpassung des Portefeuilles an die durch die Besteuerung der Kapitalerträge veränderten Anlagebedingungen Gleichung (E.10):

$$
\frac{d a}{d \tau}=\frac{a}{1-\tau} \cdot\left[1-\frac{r(1-\tau)}{1+r(1-\tau)} \cdot\left(\frac{d A}{A}: \frac{d W_{0}}{W_{0}}\right)\right] .
$$

Werden neben den Erträgen aus der riskanten Anlageform auch die sicheren zinserträge besteuert, so hat dies zur Folge, daß das Nutzenniveau vor steuern auch nach erfolgten Portefeuille-Umschichtungen nicht mehr erreichbar ist. Zu dem im Fall $r=0$ aufgetretenen substitutionseffekt kommt hier als zweiter noch ein Effekt hinzu, der zum Ausdruck bringt, daß die Nutzenbewertungen der Portefeuille-strukturen auf verschiedenen Nutzenniveaus unterschiedlich sein können. Dem gesunkenen Nutzenniveau läßt sich gedanklich ein geringeres Anfangsvermögen zurechnen. Der Effekt, der den möglicherweise unterschiedlichen Verlauf von Indifferenzkurven auf verschiedenen Nutzenniveaus zum Ausdruck bringt, wird deshalb als Vermögens- oder Einkommenseffekt bezeichnet.

1) Vgl. Cansier, D. (1985), S. 272 
In Gleichung (E.10) wird er durch die Vermögenselastizität der Nachfrage nach riskanten Anlageformen (dA/A:dWo/Wo) repräsentiert. Andert sich diese Elastizität bei unterschiedlichen Vermögensniveaus, so bedeutet dies, daß die Indifferenzkurven je nach der Vermögenshöhe unterschiedlich verlaufen.

Graphisch läßt sich diese Ronstellation analog zum Fall der ertraglosen sicheren Anlage darstellen').

Abbildung B.2: Portefeuille-struktur mit $r \neq 0$

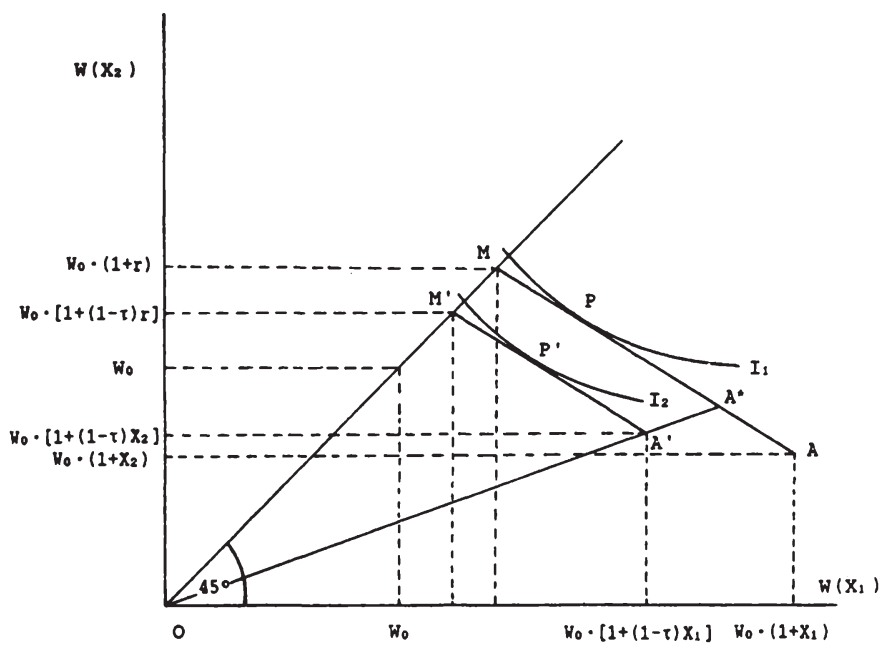

Für die riskante Anlage werden wieder zwei mögliche Realisierungen mit $X_{1}>0$ und $X_{2}<0$ angenommen. In Punkt $M(A)$ wird das gesamte Anfangsvermögen (Wo) in die sichere (riskante) Anlage investiert. Vor steuern gilt für den Anleger die Budgetlinie MA, nach Steuern reduzieren sich die möglichen Anlageerfolge auf $M^{\prime} A^{\prime}$. Vor steuern beträgt der Anteil der riskanten Anlagen MP/MA. In diesem Schaubild erhöht sich der Anteil der riskanten Anlagen nach steuern auf $M^{\prime} P^{\prime} / M^{\prime} A^{\prime}$. Der substitutionseffekt ist in der Graphik durch die Bewegung von MP/MA

1) Vgl. Stiglitz, J.E. (1969), S. 271 
nach MP/MA* dargestellt. Der Vermögenseffekt kommt in der Differenz von $M P / M A^{*}$ und $M^{\prime} P^{\prime} / M^{\prime} A^{\prime}$ zum Ausdruck, dabei ist $A^{*}$ der auf die Budgetlinie vor Steuern vom Ursprung aus übertragene Punkt $A^{\prime 1}$ ).

Für die in Abb. E.2 dargestellte Ronstellation führt die Einführung einer proportionalen steuer auf die Kapitalerträge zu einer erhöhten Risikoübernahme. Um die steuerwirkungen auf die Bereitschaft zur Obernahme von Einkommensrisiken allgemein formulieren zu können, sollen die von Pratt2) und Arrow ${ }^{3}$ ) vorgeschlagenen Maße für die Risikoaversion in die Analyse eingeführt werden.

Die absolute Risikoabneigung ist definiert durch

$$
R_{A}=-U^{\prime \prime}(W) / U^{\prime}(W) .
$$

Nimmt die absolute Risikoabneigung ab, so heißt dies, daß die Bereitschaft, ein gegebenes Risiko zu übernehmen, sich mit steigendem Vermögensniveau erhöht.

somit gilt:

(E.14)

$$
d R_{A} / d W\langle 0 \Longrightarrow d A / d W>0
$$

Das zweite Risikomaß ist die relative Risikoaversion:

$$
R_{R}=-W \cdot U^{\prime \prime}(W) / U^{\prime}(W)
$$

Nimmt die relative Risikoabneigung $z u$, so bedeutet dies, daß die Bereitschaft, ein Risiko zu übernehmen, das proportional zum Vermögen wächst, mit steigendem Vermögen abnimmt.

Somit:

$$
\begin{aligned}
\mathrm{d} R_{R} / \mathrm{dW}>0 & \Rightarrow \mathrm{da} / \mathrm{dW}<0 \\
& \Rightarrow \mathrm{dA} / \mathrm{dW}: \mathrm{A} / \mathrm{W}<1 .
\end{aligned}
$$

1) Vgl. Cansier, D. (1985), S. 272

2) Vgl. Pratt, J.W. (1964), S. $122 f f$

3) Vgl. Arrow, K.J. (1971b), S. $94 \mathrm{ff}$ 
Eine zunehmende relative Risikoabneigung ist also identisch mit einer Vermögenselastizität der Nachfrage nach riskanten Anlageformen von kleiner als eins.

Werden nun die Gleichungen (E.9) und (E.10) mit Hilfe dieser Risikomaße analysiert, so können die folgenden Aussagen abgeleitet werden ${ }^{1}$.

Eine proportionale steuer mit vollem Verlustausgleich erhöht die Bereitschaft zur Risikoübernahme, wenn

- die absolute Risikoabneigung steigt oder konstant ist; d.h.

(E.17) $\quad d R_{A} / d W \geq 0 \Longrightarrow d A / d W \leq 0$,

daraus folgt mit Gleichung (E.9):

(E.18) da/d $2 \mathrm{a} \cdot(1-\tau)^{-1}>0$;

- die relative Risikoabneigung zunimmt oder konstant bleibt; d.h.

(E.19) $\mathrm{dRR} / \mathrm{dW} \geq 0 \Longrightarrow \mathrm{dA} / \mathrm{A}: \mathrm{dW} / \mathrm{W} \leq 1$,

daraus folgt für Gleichung (E.10):

(E.20)

$$
1-\frac{r(1-\tau)}{1+r(1-\tau)} \cdot\left(\frac{d A}{A}: \frac{d W_{0}}{W_{0}}\right)>0 \Leftrightarrow \frac{d a}{d \tau}>0 .
$$

Sind absolute und relative Risikoaversion fallend, so ist keine eindeutige Aussage möglich; d.h.

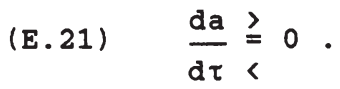

Der Anteil der risikobehafteten Titel im Portefeuille kann bei Einführung einer Ertragsteuer bzw. im Falle ihrer Erhöhung sowohl ausgeweitet als auch reduziert werden.

1) Vgl. Stiglitz, J.E. (1969), S. 274 
Bei absolut und relativ fallender Risikoaversion ist eine eindeutige Aussage über die Wirkung der Besteuerung auf die Bereitschaft zur Risikoübernahme somit nur bei Renntnis des genauen Verlaufs der Risikonutzenfunktion des jeweiligen Individuums möglich.

\section{II.} DIREKTER ANSATZ

1. Der Modellansatz

Im vorhergehenden Abschnitt wurden die wirkungen der Besteuerung auf die Bereitschaft zur Risikoübernahme in einem Portefeuille-Modell untersucht. Die Risikowirkung der Besteuerung wurde über die Veränderung des in risikobehaftete Titel investierten Vermögensanteils gemessen. Die in dieser Analyse eingenommene sichtweise entspricht derjenigen eines Rapitalanlegers, der über die Aufteilung seines Vermögens auf risikobehaftete und risikolose Anlageformen entscheidet. In diesem Abschnitt soll die Untersuchung aus der Perspektive des Investors bzw. Innovators durchgeführt werden. Mit dem folgenden Ansatz soll die Frage beantwortet werden, wie sich die Bewertung zweier vor steuern als gleichwertig erachteter Anlagemöglichkeiten unter dem Einfluß der Besteuerung der Rapitalerträge ändert'1).

Vor steuererhebung gilt:

$$
\left.U\left(Y_{0}\right)=E U(\bar{Y}) \quad 2\right) ;
$$

Yu ist das Sicherheitsäquivalent zum unsicheren Einkommen $Y$. Das Modell soll eine Aussage darüber erlauben, ob nach steuern

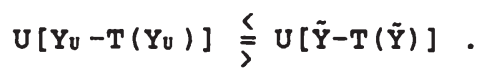

1) Die Analyse folgt den Arbeiten von Bamberg, G./Richter, W.F. (1984), S. $93 f f$ und (1985), S. 3ff; vgl. zu dieser Fragestellung auch Buchholz, H. (1985), S. $882 \mathrm{ff}$ und Schneider, D. (1977), S. $634 \mathrm{ff}$.

2) Vgl. die Marginalbedingung im Portefeuille-Modell, mit $Y=r \cdot H_{0}$ und $\tilde{Y}=X \cdot H_{0}$. 
Drei Partialeffekte müssen unterschieden werden, wenn die Besteuerungswirkungen auf vor steuern gleichwertige Alternativen bestimmt werden sollen'1).

- Der Jensen-Effekt bzw. die Proportionale Ungleichheitsaversion

$$
\alpha(Y, T)=-Y \cdot \frac{R^{\prime \prime}(Y)}{R^{\prime}(Y)}=Y \cdot \frac{T^{\prime \prime}(Y)}{1-T^{\prime}(Y)},
$$

mit

$$
R(Y)=Y-T(Y) .
$$

Der Jensen-Effekt wird gemessen durch die Einkommenselastizität des marginalen Residualeinkommens. Er wird dadurch ausgelöst, daß bei nicht-proportionaler Besteuerung konstante und schwankende Einkommen ungleich behandelt werden. Bei steuertarifen mit monoton steigenden Grenzsteuersätzen werden schwankende Einkommen steuerlich stärker belastet als konstante Einkommen:

$$
\operatorname{ET}(\tilde{Y}) \geq T[E(\tilde{Y})]
$$

(Jensens Ungleichung' )).

Steigt $\alpha(Y, T)$, so bedeutet dies eine Verschärfung der Progression. Die Folge ist eine verstärkte steuerliche Belastung unsicherer, d.h. schwankender Einkünfte. Ein höherer JensenEffekt wird deshalb einen negativen Einfluß auf die Bereitschaft zur Risikoübernahme haben.

- Der Risikoreduzierende Effekt bzw. die Residuale Einkommensprogression

$$
\rho(Y, T)=Y \cdot \frac{R^{\prime}(Y)}{R(Y)}=\frac{1-T^{\prime}(Y)}{1-t(Y)}, \quad \operatorname{mit} t(Y)=\frac{T(Y)}{Y} .
$$

1) Diese drei Partialeffekte werden bereits von Fellingham, J.C./Wolfson, M.A. (1978), S. 342 unterschieden, jedoch erst kürzlich von Bamberg, G./Richter, W.F. (1985), S. Iff formalisiert.

2) Zu dieser Ungleichung vgl. Kotz, S./Johnson, N.L. (1983), S. 297 
Als Maß für den risikoreduzierenden Effekt wird die Einkommenselastizität des Residualeinkommens verwendet. Je höher der Durchschnittsteuersatz ist, desto geringer wird die streuung des Einkommens nach Steuern') bzw. die Progression des Residualeinkommens. Entsprechend vermindert sich das Einkommensrisiko2). Je kleiner $\rho(Y, T)$ desto stärker wird der risikoreduzierende Effekt; die Risikoübernahmebereitschaft wird gefördert.

- Der Einkommenseffekt bzw. die Relative Risikoaversion

(E. 27)

$$
\alpha(Y, U)=-Y \cdot \frac{U^{\prime \prime}(Y)}{U^{\prime}(Y)} .
$$

Als Maßgröße für den Einkommenseffekt wird die Einkommenselastizität der Grenznutzen verwendet. Durch die Besteuerung wird das Einkommensniveau des Individuums und damit die lokale Risikoaversion verändert. Die Richtung dieses Effektes wird durch die einkommensniveauabhängige Risikoeinstellung bestimmt ${ }^{3}$ ).

Diese drei Teileffekte ermöglichen die folgende Aussage ) :

\section{Theorem 1:}

Die Besteuerung erhöht die Risikoübernahmebereitschaft, wenn gilt:

(E.28)

$$
\alpha(Y, U) \geq \alpha(R(Y), U) \cdot \rho(Y, T)+\alpha(Y, T)
$$

(und für mindestens ein $Y$ im betrachteten Intervall gilt das Ungleichheitszeichen streng') ) d.h.

$$
U\left[R\left(Y_{U}\right)\right] \leq E U[R(\tilde{Y})]
$$

1) Vg1. Kakwani, N.C. (1977), S. 723; siehe auch die Analyse von Richter, W.F./Hampe, J.F. (1984), S. $391 \mathrm{f}$.

2) N.B. Auch hier wird voller Verlustausgleich unterstellt.

3) Vgl. Arrow, K.J. (1971b), S. $94 f f$ und Pratt, J.W. (1964), S. $122 f$

4) Vgl. Bamberg, G./Richter, H.F. (1985), S. 6

5) Dieser Zusatz gilt auch für die weiteren Ungleichungen. 


\section{Beweis:}

Es sei

(E.30) $\quad V(Y)=U[R(Y)]$.

Somit ist zu beweisen:

(E.31) $\quad \mathrm{V}\left(\mathrm{Y}_{U}\right) \leq \mathrm{EV}(\tilde{\mathrm{Y}})$,

wobei

(E.32) $\quad \mathrm{EV}(\tilde{Y})=\mathrm{V}\left(\mathrm{Y}_{\mathrm{V}}\right)$.

$Y_{v}$ ist das Sicherheitsäquivalent nach steuern. Somit kann Gleichung (E.29) umgeformt werden $z u$ :

(E.33) $Y_{U} \leq Y_{V}$.

Nach einem Theorem von Pratt') ist (E.33) ãquivalent zu:

(E.34) $\quad \alpha(Y, U) \geq \alpha(Y, V)$.

Somit bleibt zu beweisen:

$$
\text { (E.35) } \begin{aligned}
& \alpha(Y, V)=\alpha(R(Y), U) \cdot \rho(Y, T)+\alpha(Y, T) . \\
& \Leftrightarrow \quad-Y \cdot \frac{V^{\prime \prime}(Y)}{V^{\prime}(Y)}=-R(Y) \cdot \frac{U^{\prime \prime}[R(Y)]}{U^{\prime}[R(Y)]} \cdot Y \cdot \frac{R^{\prime}(Y)}{R(Y)}-Y \cdot \frac{R^{\prime \prime}(Y)}{R^{\prime}(Y)} \\
& \Leftrightarrow \quad \frac{V^{\prime \prime}(Y)}{V^{\prime}(Y)}=\frac{U^{\prime \prime}[R(Y)] \cdot R^{\prime}(Y)}{U^{\prime}[R(Y)]}+\frac{R^{\prime \prime}(Y)}{R^{\prime}(Y)} .
\end{aligned}
$$

Mit

(E.36) $\quad V^{\prime}(Y)=R^{\prime}(Y) \cdot U^{\prime}[R(Y)]$

und

1) Pratt, J.W. (1964), S. 128 
(E.37)

$$
V^{\prime \prime}(Y)=R^{\prime}(Y)^{2} \cdot U^{\prime \prime}[R(Y)]+R^{\prime \prime}(Y) \cdot U^{\prime}[R(Y)]
$$

gilt:

(E.38)

$$
\begin{aligned}
\frac{V^{\prime \prime}(Y)}{V^{\prime}(Y)} & =\frac{R^{\prime}(Y)^{2} \cdot U^{\prime \prime}[R(Y)]+R^{\prime \prime}(Y) \cdot U^{\prime}[R(Y)]}{R^{\prime}(Y) \cdot U^{\prime}[R(Y)]} \\
& =\frac{R^{\prime}(Y) \cdot U^{\prime \prime}[R(Y)]}{U^{\prime}[R(Y)]}+\frac{R^{\prime \prime}(Y)}{R^{\prime}(Y)}
\end{aligned}
$$

$q \cdot e \cdot d$.

Das hiermit bewiesene Theorem 1 soll nun verwendet werden, um die wirkung alternativer steuerlicher Institutionen auf vor der Besteuerung als gleichwertig erachtete Handlungsmöglichkeiten zu bestimmen.

\section{2.} Proportionale Besteuerung

Bei proportionaler Besteuerung ist der Jensen-Effekt $\alpha$ ( $Y$, T) wegen der Konstanz des Grenzsteuersatzes gleich null. Ein risikoreduzierender Effekt tritt wegen der Gleichheit von Grenz- und Durchschnittsteuersatz nicht auf, also $\rho(Y, T)=1$. Damit reduziert sich Gleichung (E.28) für eine risikofördernde Wirkung der proportionalen Besteuerung auf:

$$
\alpha(Y, U) \geq \alpha(R(Y), U)
$$

$\left(E .39^{\prime}\right) \quad \Longleftrightarrow \alpha(Y, U) \geq \alpha([1-\tau] \cdot Y, U)$

Bei positivem Einkommenseffekt, d.h. steigender relativer Risikoaversion sowie auch bei konstanter relativer Risikoaversion wirkt eine proportionale Besteuerung eindeutig positiv auf die Bereitschaft zur Risikoübernahme. Bei abnehmender relativer Risikoabneigung ist keine eindeutige Aussage mög$1 \mathrm{ich} \mathrm{ch}^{1}$.

1) Vgl. dazu das Beispiel bei Feldstein, M.S. (1969b), S. $761 \mathrm{ff}$ 
Die hier abgeleiteten Bedingungen stimmen mit den im Portefeuille-Modell erhaltenen Schlußfolgerungen trotz der abweichenden Fragestellungen voll überein. Wie im folgenden gezeigt wird, können mit dem direkten Ansatz jedoch zusätzliche Fragestellungen untersucht werden, die mit dem PortefeuilleModell nicht analysiert werden können.

$\underline{3 .}$ Progressive Besteuerung

Ist ein Progressionstarif dadurch definiert, daß der Durchschnittsteuersatz mit wachsendem Einkommen steigt, so sind zahlreiche Tarife denkbar, die diese Bedingung erfüllen. Aus der Vielzahl möglicher Ronstellationen sollen zwei Fälle ausgewählt werden.

Die Ausführungen zur proportionalen Besteuerung können nicht unmittelbar auf indirekt progressive Tarife vom Typ

$$
T(Y)=\tau \cdot Y-a
$$

übertragen werden. Grund dafür ist die Tatsache, daß für $\mathrm{Y} S(\mathrm{a} / \tau)$ die steuerzahlungen negativ, d.h. zu positiven Transfers werden. Wird deshalb der betrachtete Einkommensbereich auf Einkommen mit $Y Z(a / \tau)$ beschränkt, so daß $R(Y) \leq Y$, gelten für eine indirekt progressive steuer die gleichen Aussagen wie für proportionale steuern. Für $Y Z(a / \tau)$ erhöht eine indirekt progressive steuer die Risikobereitschaft bei konstanter oder steigender relativer Risikoaversion. Ist $Y<(a / \tau)$, so ergibt sich auch bei steigender relativer Risikoaversion kein eindeutiges Ergebnis. Für eine konstante relative Risikoaversion gilt in Gleichung (E.39') nur das Gleichheitszeichen. Somit werden risikobehaftete Investitionen weder gefördert noch diskriminiert.

Bei Risikoneutralität ist wegen $U^{\prime \prime}(Y)=0$ auch der Einkommenseffekt $\alpha(Y, U)=0$. Damit ist für die Risikowirkung nur noch der Jensen-Effekt entscheidend. Dieser ist positiv für alle konvex-progressiven Tarife. Somit wird bei risikoneutralem Verhalten die Risikoübernahmebereitschaft durch die Einführung eines konvex-progressiven Tarifs gedämpft. 
4. Die Wirkungen von Tarifreformen auf die Risikobereitschaft

Der in den vorangegangenen Abschnitten vorgestellte direkte Ansatz kann auch zur Beurteilung des Einflusses alternativer Steuertarife auf die Risikobereitschaft eingesetzt werden ${ }^{1}$.

\section{Theorem 2:}

Der Tarif $T_{2}$ erhöht die Risikoübernahmebereitschaft im Vergleich zum bisher geltenden Tarif $T_{1}$, wenn gilt:

$$
\begin{aligned}
& \alpha\left(R_{1}(Y), U\right) \cdot \rho\left(Y, T_{1}\right)+\alpha\left(Y, T_{1}\right) \\
& \left.\sum \alpha\left(R_{2}(Y), U\right) \cdot \rho\left(Y, T_{2}\right)+\alpha\left(Y, T_{2}\right) 2\right) .
\end{aligned}
$$

Da Steuertarife regelmäßig aus verschiedenen Tarifstufen zusammengesetzt sind, können generelle Ergebnisse normalerweise nicht abgeleitet werden.

Die Wirkung einer in der steuerpolitischen Praxis häufig anzutreffenden Tarifänderung soll hier für einen indirekt progressiven Tarif kurz analysiert werden ${ }^{3}$.

$$
T_{2}(Y)=T_{1}(Y)-a, \text { mit a }>0 \text {. }
$$

Die Tarifreform hat also eine Erhöhung des Grundfreibetrags zum Gegenstand.

Da $T^{\prime \prime}(Y)=0$, ist auch der Jensen-Effekt gleich null. Somit erhöht die Tarifreform die Risikobereitschaft, wenn gilt:

$$
\alpha\left(R_{1}(Y), U\right) \cdot \rho\left(Y, T_{1}\right) \geq \alpha\left(R_{2}(Y), U\right) \cdot \rho\left(Y, T_{2}\right)
$$

Mit

$(E .44)$

$$
\alpha\left(R_{1}(Y), U\right)=-Y \cdot \frac{U^{\prime \prime}\left[R_{1}(Y)\right]}{U^{\prime}\left[R_{1}(Y)\right]}
$$

1) Vgl. Bamberg, G./Richter, H.F. (1985), S. $13 \mathrm{ff}$ und dieselben (1986), S. Iff sowie Richter, W.F. (1985), S. 1070

2) Der Beweis erfolgt analog zu dem von Theorem 1; vgl. auch Bamberg, G./Richter, W.F. (1986), S. 3ff.

3) Vgl. die Tarifreformen $1975 / 1978$ und 1978/1979 in der Bundesrepublik Deutschland. 
und

$$
\text { (E.45) } \quad \rho\left(Y, T_{1}\right)=Y \cdot \frac{R^{\prime} 1(Y)}{R_{1}(Y)}
$$

muß gelten:

(E.46)

$$
-Y \cdot \frac{U^{\prime \prime}\left[R_{1}(Y)\right]}{U^{\prime}\left[R_{1}(Y)\right]} \cdot Y \cdot \frac{R^{\prime}{ }_{1}(Y)}{R_{1}(Y)} \geq-Y \cdot \frac{U^{\prime \prime}\left[R_{2}(Y)\right]}{U^{\prime}\left[R_{2}(Y)\right]} \cdot Y \cdot \frac{R^{\prime}{ }_{2}(Y)}{R_{2}(Y)}
$$

Wegen

(E. 47)

$$
T_{1} \cdot(Y)=T_{2} \cdot(Y)
$$

gilt:

(E.48)

$$
R_{1}{ }^{\prime}(Y)=R_{2}{ }^{\prime}(Y) \text {; }
$$

außerdem ist:

$$
R_{2}(Y)=R_{1}(Y)+a
$$

(E. $\left.49^{\prime}\right)$

$$
\Longleftrightarrow R_{1}(Y)<R_{2}(Y) \text {. }
$$

Somit gilt Gleichung (E.41) immer dann, wenn:

$$
\text { (E.50) } \quad-\frac{U^{\prime \prime}\left[R_{1}(Y)\right]}{U^{\prime}\left[R_{1}(Y)\right]} \geq-\frac{U^{\prime \prime}\left[R_{2}(Y)\right]}{U^{\prime}\left[R_{2}(Y)\right]},
$$

d.h. die absolute Risikoabneigung monoton abnimmt' '. Die Erhöhung des Grundfreibetrags fördert die Risikoübernahme im Falle eines indirekt progressiven Tarifs, wenn die Bereitschaft der steuerpflichtigen, ein bestimmtes Risiko zu übernehmen, mit steigendem Einkommen zunimmt.

1) Dieses Ergebnis leitet Ahsan, S.M. (1974), S. 325 in einem Portefeuille-Modell ab. 


\section{UNVOLLSTANDIGER VERLUSTAUSGLEICH}

Die bisherigen Ableitungen erfolgten unter der Annahme eines sofortigen und vollständigen Verlustausgleichs. In der Realität sehen die Einkommensteuertarife jedoch regelmäBig nur einen partiellen Verlustausgleich vor. Beispielsweise ist nach \$ 10d EStG nur bis zu einem bestimmten Einkommen $V_{0} \leq 0$ ein sofortiger Verlustausgleich möglich.

Somit gilt für das steueraufkommen bei partiellem verlustausgleichl) :

(E.51) $\quad T_{P}(Y)= \begin{cases}\tau(Y) \cdot Y & \text { für } Y \geq V_{0} \\ \tau\left(V_{0}\right) \cdot V_{0} & \text { für } Y<V_{0},\end{cases}$

während bei vollständigem Verlustausgleich gilt:

(E.52) $\quad \operatorname{Tv}(Y)=\tau(Y) \cdot Y$.

Somit ist

(E.53) Tv $(Y) \leq T_{P}(Y)$

und

(E.54) $\quad \operatorname{Rv}(Y) \geq \operatorname{RP}(Y)$.

Analog zur Analyse der Wirkungen einer Tarifreform auf die Bereitschaft zur Risikoübernahme mit Hilfe von Theorem 2 kann auch ein steuertarif mit vollständigem verlustausgleich einem Tarif mit nur partiellem Verlustausgleich gegenübergestellt werden.

Partieller Verlustausgleich senkt gegenüber vollständigem Verlustausgleich die Risikobereitschaft, wenn gilt:

(E.55)

$$
\begin{aligned}
& \alpha\left(R_{v}(Y), U\right) \cdot \rho\left(Y, T_{v}\right)+\alpha\left(Y, T_{v}\right) \\
& Z \alpha\left(R_{P}(Y), U\right) \cdot \rho\left(Y, T_{P}\right)+\alpha\left(Y, T_{P}\right) .
\end{aligned}
$$

1) Vgl. Bamberg, G./Richter, W.F. (1984), S. 94 
Aus den Gleichungen (E.51) - (E.54) wird deutlich, daß bei Verlusten, die über vo hinausgehen, die streuung der Ergebnisse durch die Besteuerung nicht reduziert wird. Somit ist die streuung der Einkommen nach steuern bei partiellem Verlustausgleich größer als bei vollständigem'). Also sind die proportionale Ungleichheitsaversion (Jensen-Effekt) $\alpha(Y, T$ ) und die residuale Einkommensprogression $\rho(Y, T)$ bei partiellem Verlustausgleich größer als bei vollständigem.

Somit kann unter Berücksichtigung von Gleichung (E.54) folgende Aussage gemacht werden. Bei konstanter und abnehmender relativer Risikoaversion, $d . h . d R_{R} / d Y \geq 0$ und mit Gleichung (E.54):

$$
\alpha\left(R_{v}, U\right) \leq \alpha\left(R_{p}, U\right)
$$

vermindert ein partieller gegenüber einem vollständigen verlustausgleich die Bereitschaft zur Risikoübernahme. Für den Fall steigender relativer Risikoabneigung ist keine eindeutige Aussage möglich'). Da für Innovationen Anlaufverluste typisch sind, sind die steuerlichen Verlustausgleichsregelungen von zentraler Bedeutung für die Innovationsbereitschaft. Sie wirken über die Beeinflussung der streuung der Periodenergebnisse nicht nur auf die Bereitschaft zu Risikoübernahme, sondern bestimmen über ihre Liquiditätswirkungen auch die Möglichkeiten der Innovationsfinanzierung.

\section{DIE BEHANDLUNG SCHWANRENDER EINROMMEN}

In der bisherigen Untersuchung wurde die steuerliche Beeinflussung der Risikobereitschaft nur innerhalb einperiodischer Entscheidungsmodelle betrachtet. Anlage- und Investitionsentscheidungen haben jedoch regelmäßig Auswirkungen über mehrere Perioden hinweg. Deshalb sind als weiterer Aspekt die mehrperiodischen Besteuerungswirkungen $\mathrm{zu}$ beachten.

1) Vg1. Domar, E.D./Musgrave, R.A. (1944), S. $403 \mathrm{ff}$

2) Vgl. Cansier, D. (1985), S. 276 
Es werden wieder eine sichere und eine risikobehaftete Anlage verglichen, die vor steuern gleich bewertet werden. Die sichere Investition bringt in jeder Periode den gleichen Ertrag. Wird von steuerrechtsänderungen abgesehen, löst dieser im Zeitablauf konstante Einkommensstrom auch konstante steuerzahlungen aus. Bei risikobehafteten Engagements sind die Einkommensströme unsicher und nur zufällig im Zeitablauf konstant. Sehr viel wahrscheinlicher sind im Zeitablauf schwankende Einkommen. Sobald die Besteuerung nicht mehr streng proportional erfolgt, d.h. der Tarif nicht durchgehend proportional ist und/oder kein sofortiger Verlustausgleich besteht, haben schwankende Einkommen auch schwankende Durchschnittsteuersätze zur Folge. Werden die zinsproblematik und das Bestehen von bilanzpolitischen Manövriermassen einmal ausgeklammert'), so bedeutet dies für progressive Tarife, daß bei gleichen Gesamteinkommen über die betrachtete Periode ein schwankender Einkommensstrom steuerlich stärker belastet wird als ein konstanter, und zwar um so stärker, je größer die Einkommensschwankungen sind.

Formal bedeutet dies, für:

(E.56) $\quad \sum_{1} Y_{1}=\sum_{j} Y_{J}$

mit $Y_{1}$ : sicheres Einkommen

$Y_{j}$ : risikobehaftetes Einkommen

und

$$
\frac{d[\tau(Y)]}{d Y}>0
$$

ist

(E.57)

$$
\sum_{1} \tau(Y) \cdot Y_{1} \leq \sum_{j} \tau(Y) \cdot Y_{j}
$$

Wird der Betrachtungszeitpunkt gewechselt und dieser Aspekt ex ante, also im Entscheidungszeitpunkt untersucht, so zeigt sich, daß die steuerliche Behandlung schwankender Einkommen kein neues Problem ist, sondern nur eine Ergänzung des bereits analysierten Vergleichs eines proportionalen mit einem progressiven Tarif. Der Entscheidungsträger berūcksichtigt dieses Problem bereits bei seiner Erwartungswertbildung.

1) Vgl. dazu Wagner, F.W./Dirrigl, H. (1980), S. $277 f f$ 
Es treten somit die gleichen Risikowirkungen auf, wie sie für progressive Tarife abgeleitet wurden. Insbesondere hat die Einführung eines progressiven Tarifs wegen des Jensen-Effekts bei risikoneutralem Entscheidungsverhalten eine Dämpfung der Risikobereitschaft zur Folge.

\section{DIE BEDEUTUNG DER STEUERBILANZPOLITIR}

In den bisherigen Ausführungen wurde unterstellt, daß die periodischen Zahlungsüberschüsse die Bemessungsgrundlage der Besteuerung bilden. Eine solche Cash-flow-Besteuerung wird zwar vielfach diskutiert, entspricht jedoch nur sehr eingeschränkt dem geltenden Einkommensteuerrecht.

Das deutsche Einkommensteuerrecht unterscheidet zwei Arten der Einkommensermittlung ( $\$ 2$ I und II EStG). Während Einkünfte aus Land- und Forstwirtschaft, Gewerbebetrieb und selbständiger Arbeit als Gewinn ermittelt werden, sind die Einkünfte aus nichtselbständiger Arbeit, Rapitalvermögen, Vermietung und Verpachtung sowie die sonstigen Einkünfte als Uberschuß der Einnahmen über die Werbungskosten zu bestimmen. \$ 4 I EStG definiert den Gewinn als die Differenz zwischen dem Vermögen am Ende und dem Vermögen zu Beginn des Besteuerungszeitraums. Der Regelfall der Gewinnermittlung im deutschen Einkommensteuerrecht ist somit eine Beständerechnung. Für nicht buchführungspflichtige Gewinnermittler sowie für Uberschußermittler gilt jedoch eine modifizierte Cash-flow-Besteuerung. Einkünfte sind hier der Oberschuß der Betriebseinnahmen über die Betriebsausgaben bzw. der Einnahmen über die Werbungskosten (\$\$ 4 III bzw. 2II i.V.m. 8 und 9 EStG). Die Modifikationen bestehen darin, daß die Ausgaben für abnutzbare Vermögensgegenstände nicht direkt, sondern nur über $\mathrm{Ab}-$ schreibungen einkunftsmindernd wirksam werden (\$\$ 4III und 9I Ziff. 7 EStG). Zudem dürfen bei der Gewinnermittlung die Ausgaben für nicht abnutzbare Wirtschaftsgüter erst im Zeitpunkt ihres Ausscheidens aus dem Betriebsvermögen als Aufwand geltend gemacht werden ( $\$$ 4III EStG).

Wird von einer reinen Zahlungsstrombesteuerung abgesehen und stattdessen zumindest teilweise eine Beständerechnung durch- 
geführt, so ergibt sich bei der Vermögensbilanzierung die Notwendigkeit der Periodenabgrenzung. Die diesbezüglichen steuerrechtlichen vorschriften sehen aus verschiedensten Gründen' ( zahlreiche Ansatz- und Bewertungswahlrechte explizit vor '). Zudem haben sich durch Rechtsprechung und Verwaltungspraxis faktisch weitere Wahlrechte ${ }^{3}$ ) ergeben. Durch Ausübung dieser Wahlrechte verfügt der steuerpflichtige über eine von ihm gestaltbare steuerliche Manövriermasse, die er nutzenmaximierend einsetzen kann ${ }^{4}$ ).

Sind die nichtsteuerlichen Entscheidungen getroffen und ihre Auswirkungen bekannt, dann hat der steuerpflichtige beim Einsatz seiner Manövriermasse zwei Effekte zu beachten'). Um den Jensen-Effekt, also die stärkere steuerliche Belastung schwankender Einkommmen bei progressiven Tarifen zu verhindern, muß er versuchen, im Planungszeitraum möglichst einen konstanten Periodengewinn auszuweisen. Gleichzeitig können jedoch durch eine Vorverlagerung der Aufwendungen steuerzahlungen in die zukunft verschoben werden und so zinsvorteile erzielt werden. Unter Berücksichtigung dieser beiden Effekte ist die steuerliche Manövriermasse dann optimal eingesetzt, wenn der Barwert der Grenzsteuersätze in den Perioden des Betrachtungszeitraums konstant ist' '.

In der Realität sind aber die Auswirkungen der nichtsteuerlichen Entscheidungen regelmäßig erst ex post bekannt. Der Einsatz der Manövriermasse nach den oben genannten Rriterien ist deshalb immer nur rückwirkend für den abgelaufenen Besteu-

1) Vgl. Börner, D./Krawitz, N. (1977), S. $42 \mathrm{ff}$

2) Vgl. Bauer, J. (1981), S. $282 \mathrm{ff}$

3) Daneben besteht eine außerordentlich große Anzahl von außerbilanziellen Rechtswahlmöglichkeiten, durch deren Einsatz das steuerliche Ergebnis ebenfalls gestaltet werden kann; vgl. Michels, R. (1982), S. $182 \mathrm{ff}$.

4) Zu Grundsătzen für die Ausübung von Wahlrechten im Rahmen der Bilanzpolitik vgl. Rose, G. (1979/80), S. 49ff, Wagner, F.H./Dirrigl, H. (1980), S. $277 \mathrm{ff}$ und Haberstock, L. (1984), S. $317 \mathrm{ff}$.

5) Vgl. Wagner, F.W./Dirrigl. H. (1980), S. $282 \mathrm{ff}$

6) Bei konstanten Steuersätzen entfällt der Progressionseffekt und die optimale strategie besteht in einer möglichst starken Vorverlagerung der Aufwendungen. 
erungszeitraum unter Berücksichtigung der Unternehmensentwicklung in der laufenden Periode möglich'). Zudem ist auch die Ausübung von Wahlrechten in ihrer Wirkung nicht allein auf die jeweils betrachtete Periode beschränkt. Die Entscheidung für eine bestimmte option verursacht Bindungswirkungen auch für zukünftige Perioden. Für den steuerpflichtigen ist deshalb insbesondere die Fragestellung interessant, wie er ex ante durch bilanzpolitische Maßnahmen den Erwartungswert und die streuung der Ergebnisse seiner wirtschaftlichen Aktivitäten beeinflussen kann. In diesem Zusammenhang ist die Wahl des Abschreibungsverfahrens von besonderer Bedeutung. Für jeden Gegenstand des abnutzbaren Anlagevermögens besteht ein Methodenwahlrecht; die im Jahr der Anschaffung oder Herstellung ausgeübte Option ist aber für die gesamte Nutzungsdauer des Abschreibungsobjekts bindend.

vier steuerlich zulässige Abschreibungsverfahren werden betrachtet:

- Lineare Abschreibung:

Abschreibung in gleichen Jahresbeträgen (vgl. \$ 7 I EStG)

- Geometrisch-degressive Abschreibung:

Abschreibung eines konstanten Anteils vom (Rest-) Buchwert (vgl. \$ 7II EStG)

- Degressiv-lineare Abschreibung:

Wechsel von geometrisch-degressiver $\mathrm{zu}$ linearer Abschreibung sobald die jährlichen Abschreibungsbeträge bei linearer Abschreibung des Restbuchwerts größer sind als bei Anwendung der geometrisch-degressiven Methode (vgl. \$7III EStG)

1) Durch die Nutzung von Fristverlängerungen für die Abgabe von Steuererklärungen, die in einem vereinfachten Verfahren bereits zu einer Erklärungsfrist von 14 Monaten seit dem Ablauf des Kalenderjahres führen (vgl. die gleichlautenden Erlasse der obersten Finanzbehörden der Länder, BStBl. 1986I, S. $29 f$ und \$ 1091 AO) und die Möglichkeit der Bilanzånderung nach $\$$ II EStG (vgl. dazu Michels, R. (1982), S. 189f) kann in der steuerbilanzpolitik auch das jeweils folgende Ralenderjahr einbezogen werden und damit die Gestaltung für zwei Jahre rückwirkend vorgenommen werden. 
- Sofortabschreibung:

Absetzung der Anschaffungs- oder Herstellungskosten im Jahr der Anschaffung oder Herstellung in voller Höhe. (Nach $\$$ 6 II EStG auf Wirtschaftsgüter mit Anschaffungs-oder Herstellungskosten bis 800 DM begrenzt; da die sofortabschreibung jedoch in vielen Besteuerungskonzepten vorgesehen wird, soll sie in die Untersuchung ohne Begrenzung einbezogen werden.)

In einer simulationsstudie untersucht Rromschröder" " die Auswirkungen einer alternativen Anwendung der steuerlich zulässigen Abschreibungsmethoden auf das Gewinnziel (präzisiert durch das erwartete Endvermögen) einerseits und das Risikobzw. Sicherheitsziel (gemessen an der streuung des Endvermögens) andererseits"2).

Das Standardmodell wird durch folgende Angaben charakterisiert:

- Betrachtet wird eine Einzelunternehmung. Sie ist die einzige Einkommensquelle des ledigen Unternehmers.

Die Entnahmen belaufen sich auf 30.000 DM pro Periode.

- Das Abschreibungsobjekt wird eigenfinanziert; der einheitliche Ralkulationszinsfuß beträgt 10 prozent.

- Der Planungshorizont erstreckt sich über 20 Jahre.

- Unsicherheit besteht bezüglich der Ertragslage sowie bezüglich der effektiven Nutzungsdauer des Abschreibungsobjekts.

Für die Simulation werden zunächst reine zwei-Punkt-Verteilungen angenommen ${ }^{3}$ ). Die Einnahmen betragen 30.000 DM oder 130.000 DM. Die effektive Nutzungsdauer beläuft sich auf fünf oder zehn Jahre, während die steuerliche Nutzungsdauer acht Jahre beträgt.

- Wird das Abschreibungsobjekt während des Planungszeitraums ersetzt, erhöht der Veräußerungsgewinn das Einkommen im Jahr des Ausscheidens des Anlageguts.

- Die Anschaffungs- oder Herstellungskosten werden zwischen $1.000 \mathrm{DM}$ und $180.000 \mathrm{DM}$ variiert.

1) Kromschröder, B. (1983)

2) Kromschröder, B. (1983), S. 54

3) D.h. beide Ausprăgungen haben die gleiche Eintrittswahrscheinlichkeit. 
- In die Analyse einbezogen werden die Einkommen- und Gewerbesteuer. Verlustvortrag und -rücktrag nach \$ 10d EStG sind in der studie nicht berücksichtigt').

Tabelle E.1: Rangfolge der Abschreibungsverfahren

\begin{tabular}{|c|c|c|c|c|c|c|c|c|c|c|c|c|c|c|c|c|c|c|c|c|c|}
\hline \multicolumn{22}{|c|}{ Vorteilhaftigkeit in Binblick auf } \\
\hline & \multicolumn{7}{|c|}{ erwartetes Bndrernoggen } & \multicolumn{7}{|c|}{ Risiko } & \multicolumn{7}{|c|}{ beides } \\
\hline Verfahren & a & $b$ & c & d & & ges* & 1 & a & $b$ & $c$ & $d$ & e & gest & t & a & $b$ & $c$ & $d$ & e & ge & 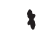 \\
\hline degressiv & 6 & 3 & 4 & 3 & & 22 & 7 & 16 & 7 & 7 & 5 & 4 & 39 & 13 & 2 & 1 & 3 & 2 & 3 & 11 & 4 \\
\hline degressiv-linear & 18 & 46 & 15 & 38 & 27 & 174 & 60 & 12 & 3 & 2 & 1 & 1 & 19 & 6 & 1 & 3 & 2 & 0 & 0 & 9 & 3 \\
\hline linear & 0 & 0 & 7 & 15 & 3. & 45 & 15 & 41 & 47 & 50 & 49 & 49 & 236 & 79 & 0 & 0 & 6 & 13 & 21 & 40 & 14 \\
\hline Sofort-Abschr. & 39 & 9 & 3 & 0 & & 52 & 18 & 1 & 0 & 0 & 2 & 2 & 5 & 2 & 0 & 0 & 0 & 0 & 0 & 0 & 0 \\
\hline Sunen & 63 & 58 & 59 & 56 & $5 \%$ & 293 & 100 & 70 & 57 & 59 & 57 & 56 & 299 & 100 & 6 & 4 & 11 & 15 & 24 & 60 & 100 \\
\hline
\end{tabular}

Anschaffungs- oder Herstellungskosten:
a $20.000 \mathrm{DM}$
c $100.000 \mathrm{DM}$
e $180.000 \mathrm{DM}$
b $60.000 \mathrm{DM}$
d $140.000 \mathrm{DM}$

* Wegen Gleichrangigkeiten sind spaltensummen größer als die Anzahl der Fälle (290)

Quelle: Rromschröder, B. (1983), S. 50

1) Zu weiteren Einzelheiten vgl. Kromschröder, B. (1983), S. Iff 
In der Untersuchung werden 290 Fälle ausgewertet. Für jeden Fall werden 1.000 Zustandsfolgen simuliert, um repräsentative Verteilungen des Endvermögens zu erhalten.

Die Auswertung der 290 Fallbeispiele führt zu folgenden Ergebnissen (Tab. E.1):

In 60 Prozent der untersuchten Fäle liefert die degressivlineare Abschreibungsmethode den höchsten Erwartungswert des Endvermögens, gefolgt von der Sofortabschreibung mit 18 Prozent und der linearen Abschreibung mit 15 Prozent.

Im Hinblick auf die streuung der Vermögensendwerte schneidet das lineare Abschreibungsverfahren am günstigsten ab; in 79 Prozent der Fälle führt sie zur geringsten standardabweichung. An zweiter stelle steht hier die degressive Abschreibung mit 13 Prozent vor der degressiv-linearen Methode mit 6 Prozent.

Eine differenziertere Bewertung der Ergebnisse wird möglich, wenn Erwartungswert und Standardabweichung in Abhängigkeit von der Höhe der Anschaffungs- oder Herstellungskosten betrachtet werden. Im Bereich niedriger Anschaffungs- und Herstellungskosten ist die Sofortabschreibung im Hinblick auf das erwartete Endvermögen die günstigste Alternative. Bei Anschaffungs- und Herstellungskosten von mehr als 20.000 DM wird mit der degressiv-linearen Abschreibungsmethode das höchste erwartete Endvermögen erreicht; allerdings gewinnt bei hohen Anschaffungsausgaben die lineare Methode immer mehr an Bedeutung.

In bezug auf das Risiko ist das lineare Abschreibungsverfahren durchweg die vorteilhafteste Methode. Bei niedrigen Anschaffungs- oder Herstellungskostem gewinnen die degressive und die degressiv-lineare Abschreibung etwas an Bedeutung.

Die Simulationsergebnisse werden nach einigen Oberlegungen schnell einsichtig. Besteht nur Unsicherheit bezüglich der Ertragslage, dann wird die streuung der Periodenergebnisse bei linearer Abschreibung des Investitionsobjekts, d.h. bei Absetzung eines konstanten Betrages während der steuerlichen Nutzungsdauer, nicht beeinflußt. Der Erwartungswert wird um den Abschreibungsbetrag reduziert, die standardabweichung bleibt unverändert. 
Die Varianz der Periodenergebnisse vor Abschreibungen $Y$ betrăgt:

$$
(E .58) \quad \sigma^{2}=E\left[(Y-E(Y))^{2}\right] .
$$

Durch die Abschreibungen d wird der Bruttogewinn um einen konstanten Betrag in jeder Periode reduziert:

$$
\sigma_{d}^{2}=E\left[((Y-d)-E(Y-d))^{2}\right] .
$$

Da die Abschreibungsbetrăge bei linearer Abschreibung konstant sind, ist $E(d)=d$.

Somit beträgt die Varianz nach Abschreibungen:

$$
\text { (E.60) } \quad \begin{aligned}
\sigma_{d}^{2} & =E\left[((Y-d)-E(Y)+d)^{2}\right] \\
& =E\left[(Y-E(Y))^{2}\right] \\
& =\sigma^{2} .
\end{aligned}
$$

Bei Anwendung eines nichtlinearen Abschreibungsverfahrens werden die Absetzungen im Vergleich zur linearen Methode vorverlagert. Am stärksten ist die Verschiebung bei der Sofortabschreibung, gefolgt von der degressiv-linearen und degressiven Methode. Bei unsicheren Einkommen wirken diese Verfahren tendenziell schwankungsverstärkend. Wird zusätzlich berücksichtigt, daß die effektive Nutzungsdauer auch kürzer als die steuerliche sein kann und daß die veräußerungserlöse voll zu versteuern sind, so verursachen auch hier nichtlineare Abschreibungsverfahren höhere Ertragsschwankungen, da sie höhere stille Reserven bilden.

In bezug auf das Gewinnziel sind zwei gegenläufige Effekte zu beachten. Durch eine Vorverlagerung der Abschreibungen können steuerzahlungen in die zukunft verschoben werden. Andererseits kōnnen bei progressiven Tarifen durch die Angleichung der jährlichen Gewinnausweise steuervorteile erzielt werden. In der hier vorliegenden studie scheint der zinseffekt gegenüber dem Progressionseffekt zu überwiegen').

1) Wird ein höheres Einkommensniveau unterstellt, rückt das zu versteuernde Einkommen in den Bereich abnehmender Progression bzw. konstanter Grenzsteuersätze, sodaß der Progressionseffekt verschwindet. 
Die Sofortabschreibung sowie die degressiv-lineare Methode sind die günstigsten Alternativen, während die lineare Abschreibung bei hohen Anschaffungs- oder Herstellungskosten an Bedeutung gewinnt. Zwei Teilbereichsergebnisse bestätigen diese Einschätzung. Wird ein gespaltener Kalkulationszinsfu $\beta$ mit einem Haben-zinssatz von 5 und einem Soll-zins von 30 Prozent eingeführt, so bleibt die Rangfolge der Abschreibungsverfahren unverändert, die Bedeutung des Iinearen Verfahrens steigt jedoch spürbar an. Wird eine Obertragung von Veräußerungsgewinnen nach $\$ 6 b$ Estg zugelassen, so gewinnt insbesondere die sofortabschreibung größeres Gewicht.

Nicht berūcksichtigt sind in dieser Analyse die Verlustausgleichsmöglichkeiten nach \$ 10d EStG. Aufgrund der bisherigen Ergebnisse dürften diese im Hinblick auf das Gewinnziel die Verfahren beschleunigter Abschreibungen weiter begünstigen, sowie in bezug auf das sicherheitsziel die Attraktivität der linearen Abschreibung reduzieren.

Im besprochenen Modell wird die Risikowirkung einer einmaligen Investition im direkt progressiven Bereich des Einkommensteuertarifs untersucht.

Investiert das Unternehmen jedoch kontinuierlich, so hat es unabhängig von der Wahl der Abschreibungsmethode ein jährlich konstantes Abschreibungsvolumen zur verfügung. Sollen alle abnutzbaren Anlagegüter nach dem gleichen Verfahren abgeschrieben werden, ist für die Wahl der Methode nur das Gewinnziel relevant, d.h. in die Entscheidung gehen nur noch der zins- und der Progressionseffekt ein.

Die Entscheidungssituation wird weiter vereinfacht, wenn konstante Grenzsteuersãtze und relativ stabile Ertragserwartungen bestehen. Hier ist nur noch der zinseffekt zu beachten. 
VI. DIE WIRKUNG DER BESTEUERUNG VON VERMOGEN UND UMSATZ AUF DIE RISIROBEREITSCHAFT

Die bisherige Analyse beschränkte sich auf die wirkungen der Ertragsbesteuerung. Auch bei der Vermögensbesteuerung ist die steuerwirkung von der individuellen Risikonutzenfunktion abhängig. Wird auf das Jahresendvermögen

$$
W=[1+r+a(X-r)] \cdot W_{0}
$$

eine Vermögensteuer mit dem steuersatz $\tau$ erhoben,

$$
W_{\tau}=\left(1-\tau_{w}\right)[1+r+a(X-r)] \cdot W_{0},
$$

so wird deutlich, daß die Vermögensbesteuerung einer Reduzierung des Anfangsvermögens Wo gleichkommt. Somit wirkt eine proportionale Vermögensteuer risikofördernd, risikoneutral oder risikomindernd, je nachdem ob die Vermōgenselastizität der Nachfrage nach riskanten Anlageformen größer, gleich oder kleiner eins ist (bzw. die relative Risikoaversion fallend, konstant, steigend)1'.

Bei einem Kapitalumschlag von 100 Prozent bzw. in einem einperiodischen Modell wirkt die Umsatzsteuer wie eine Vermögensteuer ${ }^{2}$. Die Wirkung der Umsatzsteuer ist nicht für alle Unternehmen gleich. Sie ist abhängig von der Umsatzrendite. Je geringer der Gewinn ( $Y$ ) bei einem bestimmten Umsatz (U), je geringer also die Umsatzrendite $\left(r_{u}\right)$, desto stärker wird der Gewinn durch die Umsatzsteuer belastet ${ }^{3}$ ) :

(E.63) $\quad \frac{T}{Y}=\frac{\tau_{u} \cdot U}{Y}=\tau_{u} \cdot \frac{1}{r_{u}}$

(E.64) $\frac{d(T / Y)}{d Y}=-\tau_{u} \cdot \frac{U}{Y^{2}}$.

1) Vgl. Atkinson, A.B./Stiglitz, J.E. (1980), S. 104. Die Vermōgensbesteuerung läßt sich entsprechend im direkten Ansatz darstellen; vgl. Bamberg, G./Richter, H.F. (1986), S. 4.

2) Vgl. Stiglitz, J.E. (1972), S. $302 f f$

3) Vgl. Cansier, D. (1985), S. 264 


\section{DIE DISRRIMINIERUNG UNTERNEHMERISCHER TATIGREIT}

Die bisherigen Ableitungen zu den Wirkungen der Besteuerung auf die Risikobereitschaft führen jeweils zu dem Ergebnis, daß eine eindeutige Aussage über die Richtung der steuerwirkungen nur bei Kenntnis des Verlaufs der Nutzenfunktion des Entscheidungsträgers gemacht werden kann. Die einzige Ausnahme hierzu bildet der Aspekt der Verlustausgleichsregelung. Unvollständiger Verlustausgleich hat hier eindeutig eine Verminderung der Risikoübernahmebereitschaft zur Folge. Grund für dieses Ergebnis ist der Tatbestand, daß das sichere Einkommen von der Verlustausgleichsregelung nicht berührt wird, da es größer als null ist. Die Verlustausgleichsregel ist somit diskriminierend und trifft nur das risikobehaftete Einkommen.

Zu analogen Ergebnissen führen deshalb solche steuerlichen Institutionen, die einseitig nur eine Einkommensart begünstigen oder benachteiligen. Bei der Entscheidung zwischen abhängiger Beschäftigung mit weitgehend sicheren Einkommen und risikobehafteter unternehmerischer Tätigkeit haben zum Beispiel Weihnachts- und Arbeitnehmerfreibetrag, steuerfreiheit von Abfindungen, Beihilfen, Sonn-, Feiertags- und Nachtarbeitszuschlägen sowie die subventionierte Alterssicherung der Arbeitnehmer eine Diskriminierung der unternehmerischen Tätigkeit zur Folge'). Die genannten Regelungen sind von besonderer Bedeutung für Innovationsaktivitäten, da diese oft mit dem Wechsel von einer abhängigen Beschäftigung in ein selbständige Tätigkeit verbunden sind.

Umgekehrt wirken Investitionszulagen risikofördernd, da sie nur für risikobehaftete unternehmerische Tätigkeiten gewährt werden ${ }^{2}$.

1) Vgl. Institut für Wirtschaftspolitik an der Universităt zu Röln/ Ifo-Institut für Wirtschaftsforschung München (1982), S. $477 \mathrm{ff}$

2) Vgl. Schneider, D. (1983), S. 74 
Die Mehrwertigkeit der Erwartungen ist in den bisherigen Ausführungen auf das Ergebnis der wirtschaftlichen Aktivitäten vor steuern beschränkt geblieben. In der Realität tritt jedoch zu diesem kommerziellen Risiko, das vor allem durch die Unsicherheit über das Verhalten der Lieferanten, Abnehmer und Konkurrenten bestimmt wird, noch ein politisches Risiko hin$\left.z^{1}\right)$. Die Folge dieses zusätzlichen Risikoaspekts ist, daß die streuung der erwarteten Ergebnisse erhöht wird. Bei risikoaversen Entscheidungsträgern bewirkt dies, um den Erwartungsnutzen konstant zu halten, einen substitutionseffekt hin zu sicheren Anlageformen ${ }^{2}$ ) und damit einen Rückgang der Innovationstätigkeit.

An dieser stelle soll auf den steuerpolitischen Aspekt dieses politischen Risikos näher eingegangen werden. Drei Bereichen steuerpolitischen Risikos sieht sich der steuerpflichtige bei der Planung seiner wirtschaftlichen Aktivitäten gegenüber ${ }^{3}$ ).

Zum ersten besteht Unsicherheit über den Inhalt des steuerrechts im Planungszeitraum aufgrund der Unbeständigkeit kodifizierter, insbesondere steuerrechtlicher Normen ${ }^{4}$.

Der zweite Unsicherheitsbereich betrifft die Folgen inexakter Rechtsetzung und der Unbestimmtheit von Rechtsnormen. Ein besonderes Problem stellt hier der Zeitraum zwischen der Verkündung der interpretationsbedürftigen Norm und der Erläuterung und klärung durch die Finanzverwaltung und Finanzgerichte. Oft dauert der Zeitraum mehrere Jahres' und systematische Planung wird unmöglich.

Den dritten Unsicherheitsbereich bilden die steuerausweichund steuerüberwälzungsprozesse, d.h. die wirkungen der steuerlichen Institutionen auf das Verhalten der Ronkurrenten und Marktpartner. Dieser Aspekt des steuerpolitischen Risikos ist

1) Vgl. Ekern, S. (1971), S. $421 \mathrm{f}$

2) Vgl. Diamond, P.A./Stiglitz, J.E. (1974), S. $345 \mathrm{ff}$; Menezes, C.F./Hanson, D.C. (1970), S. 481ff; Zeckhauser, R./Keeler, E. (1970), S. 661ff

3) Vgl. Schneider, D. (1983), S. 65; dazu auch Rose, G. (1983), S. $100 f f$ und derselbe $(1975 / 76)$, S. $41 \mathrm{ff}$

4) Zum Ausmaß der Steuerrechtsänderungen vgl. die Beispiele bei Borell, R./Schemmel, L. (1987), S. $110 \mathrm{f}$

5) Vgl. Rose, G. $(1975 / 76)$, S. $55 f$ und $62 f$ 
allerdings vom kommerziellen Risiko kaum zu trennen.

Diese Ausführungen zeigen, daß vom politischen Bereich erhebliche zusätzliche Risiken auf die privaten wirtschaftlichen Aktivitäten ausgehen können. Durch eine Verstetigung der gesetzgeberischen Maßnahmen kann deshalb eine beträchtliche Reduzierung der Risiken erreicht werden, denen sich innovationswillige Wirtschaftssubjekte gegenüber sehen.

IX. ZUSAMMENFASSUNG

Gegenstand dieses Rapitels war die Untersuchung der steuerlichen Wirkungen auf die Bereitschaft zur Risikoübernahme. Bereits die Analyse der Portefeuille-Entscheidung über risikolose und risikobehaftete Anlagemöglichkeiten hat gezeigt, daß keine eindeutigen steuerwirkungen ableitbar sind. Dieses Ergebnis wird verstärkt, wenn zusätzlich Alternativen mit unterschiedlichem Risikograd oder verschiedene steuertarife verglichen werden. Eine Ausnahme bilden nur diskriminierende steuerliche Regelungen. Hier sind eindeutige Wirkungen zu ermitteln.

Zudem erfolgte die Analyse in einem einperiodischen Modell. Die abgeleiteten Ergebnisse lassen sich nur unter engen Restriktionen für Nutzenfunktionen') und Ertragsstruktur ${ }^{2}$ ) auf mehrperiodische Entscheidungen übertragen. Eindeutige Aussagen über die Wirkung der Besteuerung auf die Risikoübernahmebereitschaft sind in intertemporalen Modellansätzen nicht möglich ${ }^{3}$ ).

Empirische Untersuchungen der Vermögenstruktur privater Haushalted) erlauben eine Eingrenzung der möglichen steuerwirkungen in Abhängigkeit von dem Vermögensniveau der Haushalte.

1) Vgl. Drèze, J.H./Modigliani, F. (1970), S. 317ff; Hagen, K.P. $(1970 / 71)$, S. $274 f f$ und Ahsan, S.M. (1976), S. $343 \mathrm{ff}$

2) Vgl. Sandmo, A. (1969), S. 597

3) Vgl. Sandmo, A. (1985), S. 301

4) Vgl. Morin, A.-R./Fernandez Suarez, A. (1983), S. 1201ff; Friend, I./ Blume, M.E. (1975), S. 900ff; Blume, M.E./Friend, I. (1975), S. 585ff und Cohn, R.A. et al. (1975), S. $605 \mathrm{ff}$ 
Generell kann den Wirtschaftssubjekten eine abnehmende relative Risikoabneigung unterstellt werden, d.h. mit steigendem Haushaltsvermögen wächst die Bereitschaft, ein bestimmtes relatives Risiko $z$ übernehmen. Allerdings ist bei Nettovermögen über 100.000 \$ die Degression sehr gering, sodaß praktisch von einer konstanten relativen Risikoaversion ausgegangen werden kann. Nur bei Vermögen von 1 - 12.500 s ist die relative Risikoabneigung ansteigend'). Ober alle Vermögensklassen hinweg erhöht sich die Risikoaversion mit dem Alter der Haushaltsmitglieder' ${ }^{2}$.

Die Ergebnisse werden kompliziert und die Analyse wird vielfältiger, wenn die durch den einfachen, partialanalytischen Modellansatz eingeführten Restriktionen bedacht werden. Oberwälzungsprozesse wurden durch die Annahmen

$(E .65)$

$$
R(Y)=[1-\tau(Y)] Y
$$

und

(E.66) Wr $\equiv(1-\tau)$ Wo $_{0}$

ausgeschlossen. Auch Rückwirkungen aus der Verwendung der Steuermittel wurden nicht berücksichtigt. Tatsächlich werden aber durch steuererhebung und staatliche Ausgabentätigkeit zahlreiche Anpassungsreaktionen ausgelöst. Die Bestimmung der Richtung und Wirkungen dieser Prozesse setzt die Renntnis der Einkommens- und Preiselastizitäten voraus ${ }^{3}$ ).

1) Vgl. Morin, A.-R./Fernandez Suarez, A. (1983), S. $1026 \mathrm{ff}$

2) Vgl. Morin, A.-R./Fernandez Suarez, A. (1983), S. $1029 \mathrm{ff}$

3) Vgl. Stiglitz, J.E. (1972), S. $351 f$ 
F. DER EINFLUSS DER FINANZIERUNGSBEDINGUNGEN AUF DIE INNOVATIONSBEREITSCHAFT

Als ein Charakteristikum der Finanzplanung für Innovationsprojekte ist zu beachten, daß verhältnismäßig hohen Auszahlungen bis zum Zeitpunkt der Markteinführung keine oder nur geringe Einzahlungen gegenüberstehen. Bei Berücksichtigung dieser struktur der Zahlungsströme wird deutlich, daß die Innovationsfähigkeit entscheidend durch die Möglichkeiten der Kapitalbildung und Kapitalbeschaffung bestimmt wird. Innovationsbereitschaft besteht nur dann, wenn die institutionellen Rahmenbedingungen den (potentiellen) Unternehmer auch in die Lage versetzen, sich die für das Projekt erforderlichen Finanzierungsmittel zu beschaffen.

In diesem Rapitel werden die steuerlichen und marktlichen Bedingungen der Kapitalbildung und Kapitalbeschaffung analysiert. Die Untersuchung erfolgt dabei unter dem Gesichtspunkt der Akkumulation und Zufuhr liquider Mittel sowie ihrer Rosten. Auf damit zusammenhängende Fragestellungen wie beispielsweise den Einfluß der Finanzierungsstruktur und ihrer steuerlichen Folgen auf den Marktwert der Unternehmen wird demnach nicht eingegangen ${ }^{1}$ ). Zunächst werden die steuerlichen Bedingungen der Rapitalbildung analysiert. Daran anschließend werden die Möglichkeiten der Kapitalzuführung durch Dritte untersucht und die Konsequenzen für die Innovationsaktivitäten abgeleitet.

1) Vgl. dazu Modigliani, F./Miller, M.H. (1958), S. $261 \mathrm{ff}$ und dieselben (1963), S. 433ff; Farrar, D.E./Selwyn, L.L. (1967), S. 444ff und Seelbach, H. (1979), S. 692ff und zur Kritik Moxter, A. (1970), S. $128 \mathrm{ff}$ 
1. Die steuerlichen Rahmenbedingungen der Kapitalbildung in privaten Haushalten

Die Möglichkeiten der Ansammlung von Gründungskapital werden auf zwei stufen von steuerlichen Bestimmungen beeinflußt. Auf der ersten stufe steht die Beeinflussung der Höhe des anlagefähigen Betrages durch die Besteuerung. Die für Ronsumtion und Sparen verwendbaren Mittel stammen aus versteuertem Einkommen (Ausnahmen sind solche Einkommensteile, die nicht im Einkommensbegriff des EStG enthalten sind, sowie nach $\$ 3$ EStG steuerfreie Einnahmen). Somit werden die Möglichkeiten des Ronsumverzichts und damit der Kapitalbildung durch die absolute Höhe der Steuerlast sowie die Zwangsabgaben zur Sozialversicherung bestimmt.

Außer dem Volumen wird auch die Richtung der Ersparnisbildung durch steuerliche Regelungen beeinflußt. Nach $\$ 10 I$ EStG sind die Beiträge zu bestimmten Versicherungen und an Bausparkassen bei der Einkommensermittlung als Sonderausgaben abziehbar. Die in diese Verwendung geleiteten Einkommensteile bleiben somit steuerfrei.

Die steuerliche Behandlung der Rapitalerträge bildet die zweite Stufe der Beeinflussung der Kapitalbildung. Einkünfte aus Rapitalanlagen sind nach $\$ 20$ EStG als Einkünfte aus Rapitalvermögen (bzw. nach $\$ 21$ EStG als Einkünfte aus Vermietung und verpachtung) zu versteuern. Ausgenommen hiervon sind die zinsen auf die nach $\$ 10 I$ ziff. $2 b$ und 3 EStG geleisteten Beiträge sowie Zinsen auf Anleihen nach $\$ 3 a$ EStG.

Im folgenden wird diskutiert, inwieweit die Bildung von Gründungskapital im Rahmen der Maßnahmen zur Unterstützung der privaten Vermögensbildung gefördert wird. 
Nach dem Spar-Prämiengesetz (SparPG) und dem Wohnungsbau-Prämiengesetz (WOPG) werden bestimmte Formen des Rontensparens, Anteils- und Wertpapiererwerbs in Höhe von 14 Prozent der jährlichen sparbeiträge bzw. der prämienbegünstigten Aufwendungen im Sinne des WOPG gefördert'). Voraussetzung fūr die Gewährung der Prämien ist zum einen, daß bestimmte Einkommensgrenzen nicht überschritten werden ${ }^{2}$ ), und zum zweiten, daß über die prämienbegünstigten Leistungen nicht vor Ablauf einer sperrfrist von sechs oder sieben Jahren verfügt wird ${ }^{3}$ ). Die vorzeitige Verwendung der prämienbegünstigten Ersparnisse ist nur unter sehr eingeschränkten Bedingungen ohne verlust der Prämie möglich. Neben Anderungen in den persönlichen Verhältnissen ist bei einer Förderung nach dem WoPG nur die sofortige Verwendung der Mittel für den Wohnungsbau prämienunschädich. Seit dem Ralenderjahr 1984 kann das nach dem SparPG angesparte Kapital für Unternehmensgründungen verwendet werden. Nach $\S 1$ IV ziff. 2e sparPG ist eine Verwendung der Mittel vor Ablauf der sperrfrist dann prämienunschädlich, wenn der Prämiensparer seine nichtselbständige Arbeit aufgibt und eine nach $\$ 138$ I AO anzeigenpflichtige, d.h. freiberufliche oder gewerbliche Tätigkeit aufnimmt.

Der Anlagekatalog des 5. VermBG schließt die nach dem sparPG und dem WoPG geförderten sparformen ein und umfaßt zudem Leistungen zu Rapitalversicherungen. Die Arbeitnehmer-Sparzulage für vermögenswirksame Leistungen, die in Anteilen an Unternehmen sowie im Wohnungsbau angelegt werden, beträgt 23 Prozent, ansonsten 16 Prozent4). Der geförderte Betrag ist auf

1) Zu den begünstigten Leistungen vgl. \$1II SparPG und \$2 WoPG. Pro Kind wird der Prämiensatz um zwei Prozentpunkte erhöht (\$ 2 I SparPG, \$ 3 I WOPG). Maximal sind im Kalenderjahr 800 DM bzw. 1600 DM bei Ehegatten begünstigt ( $\$ 2$ IV SparPG, \$3IV WoPG).

2) Die Grenzen bilden zu versteuernde Einkommen von 24.000 DM bzw. 48.000 DM bei Ehegatten ( $\$ 1 \mathrm{a}$ SparPG, $\$ 2 \mathrm{a}$ WOPG).

3) § IIII SparPG, $\$ 2$ II WOPG

4) \$12III VermBG; bei drei und mehr Kindern erhöht sich die Zulage auf 33 bzw. 26 prozent. 
624 DM begrenzt, erhöht sich aber auf 936 DM, wenn der Differenzbetrag im Unternehmensbereich angelegt wird.

Die Darstellung der staatlichen Förderung der Vermögensbildung zeigt, daß die Gründungskapitalbildung durch das SparPrämiengesetz, das Wohnungsbau-Prämiengesetz, das Vermögensbildungsgesetz sowie die steuerlichen Vergünstigungen nur unwesentlich begünstigt wird. Dies liegt zum einen daran, daß die Förderung zweckgebunden ist und die begünstigungsfähigen Beträge einer sperrfrist unterliegen und zum anderen an der Begrenzung des Kreises der Berechtigten auf unbeschränkt steuerpflichtige mit $z u$ versteuernden Einkommen von unter 24.000 DM bzw. 48.000 DM bei Ehegatten.

\section{Die Rapitalbildung in Unternehmen}

In Personengesellschaften werden die Gewinnanteile der Gesellschafter unabhängig davon, ob sie im Unternehmen verbleiben oder entnommen werden, mit den persönlichen Einkommensteuersätzen der Gesellschafter belastet.

Bei Rapitalgesellschaften erfolgt eine Trennung von Unternehmenssphäre und Privatbereich. Die Rapitalgesellschaft ist selbständiges steuersubjekt. Nicht entnommene Gewinne werden dem Thesaurierungssatz der Rörperschaftsteuer, also dem Spitzensatz der Einkommensteuer unterworfen. Werden nun die beiden Ertragsteuern zusammengefaßt, so stehen bei einem Hebesatz der Gewerbesteuer von 350 Prozent 37,45 Prozent des thesaurierten Gewinns für die Finanzierung von Investitionsvorhaben zur Verfügung. Wird eine Rapitalrendite von 10 Prozent unterstellt, reduziert sich der verfügbare Betrag nach Ertrag- und substanzsteuern auf 30,33 Prozent, d.h. es muß das 3,3-fache des zur Selbstfinanzierung vorgesehenen Betrages verdient werden'1).

1) Vgl. Bierich, M. (1983), S. $41 \mathrm{ff}$ 

RAPITALBESCHAFFUNG

1. Die steuerliche Behandlung alternativer Finanzierungsformen

Um die Wirkungen der Besteuerung auf die Auswahl von Finanzierungsalternativen $z u$ ermittel $n^{11}$, werden mittels eines paarweisen Vergleichs die steuerlichen Rosten der Finanzierungsformen einander gegenübergestellt. Im folgenden wird die Rechtsform der Kapitalgesellschaft unterstellt und zwischen Fremdfinanzierung, Beteiligungs- und selbstfinanzierung unterschieden. Bei Personengesellschaften fallen auf der Gesellschaftsebene keine steuern an und es ist nur zwischen Fremdfinanzierung und Selbstfinanzierung zu unterscheiden.

$\underline{1.1}$ Die steuerlichen Implikationen der einzelnen Finanzierungsformen

\subsubsection{Fremdfinanzierung}

Wie jede andere Finanzierungsmaßnahme führt auch die Fremdkapitalaufnahme $z u$ einer Erhöhung der Aktiva eines Unternehmens. Dieser Vergrößerung der substanzsteuerlichen Bemessungsgrundlage $(\delta K)$ steht aber entgegen, da $\beta$ die eingegangenen Verbindlichkeiten (FR), soweit sie Betriebsschulden sind, als Schuldposten bei der Ermittlung der substanzsteuerlichen Bemessungsgrundlage wieder abzuziehen sind2).

Für die Vermögensteuerbelastung ( $S^{v}$ ) ergibt sich bei einem Steuersatz $\mathbf{s}^{v}$ :

$$
S^{v}=S^{v}(\delta R-F R) \text {. }
$$

Bei der Gewerbekapitalsteuer ist zu beachten, daß ein Teil der Betriebsschuld bei der Ermittlung des Gewerbekapitals als Dauerschuld (DS) wieder hinzuzurechnen ist:

1) Zur Behandlung dieses Problems in der Finanzierungstheorie vgl. Farrar, D.E./Selwyn, L.L. (1967), S. $444 \mathrm{ff}$

2) Zum Folgenden vgl. Wagner, F.W./Dirrigl, H. (1980), S. $82 \mathrm{ff}$ 
(F.2)

$S^{g k}=S^{g k}(\delta R-F R+D S)$.

Bei der Bestimmung der Ertragsteuerbelastung sind die auf das Fremdkapital zu leistenden zinszahlungen (ZI) ertragsmindernd anzusetzen. Für die Gewerbeertragsteuer sind diese jedoch teilweise als Dauerschuldzinsen (DSZI) wieder hinzuzurechnen. Zudem ist die Gewerbekapitalsteuer von der Bemessungsgrundage der Gewerbeertragsteuer abzugsfähig. Daß die Gewerbeertragsteuer von ihrer eigenen Bemessungsgrundlage abzugsfähig ist, wird bereits bei der Ermittlung des steuerfaktors für den Gewerbeertrag soe berücksichtigt'). Ist FF der Ertrag, der erforderlich ist, um die mit der Fremdfinanzierung verbundenen steuerlichen Kosten sowie die Zinszahlungen zu dekken, beträgt die Belastung mit Gewerbeertragsteuer so :

$$
\text { Sge }=s^{g e}(F F-Z I+D S Z I-S g k) \text {. }
$$

Die Körperschaftsteuerbelastung $\mathbf{S}^{k n}$ beträgt unter Berücksichtigung der Abzugsfähigkeit der Gewerbesteuer:

$$
S^{k n}=S^{k n}\left(F F-Z I-S_{g e}-S_{g k}\right) .
$$

Auf der Ebene der Kapitalgeber sind die zinszahlungen Gegenstand der persönlichen Einkommensteuer und die Forderung gegen das Unternehmen ist Teil der Bemessungsgrundlage der (persönlichen) Vermögensteuer.

\subsubsection{Beteiligungsfinanzierung}

Wie die Fremakapitalaufnahme führt auch die Rapitalzuführung durch Ausgabe von Gesellschaftsrechten zu einer Erhöhung des Kapitalstocks. Eigenkapitalanteile sind aber keine schuldposten und deshalb nicht von der substanzsteuerlichen Bemessungsgrundlage abzugsfähig. Somit belaufen sich die substanzsteuern auf'):

1) Betrăgt der Gewinn vor Abzug der Gewerbeertragsteuer $G$ und der Hebesatz H, ergibt sich der Gewerbesteuerfaktor $\mathrm{s}^{g e}=0,05 \cdot(\mathrm{H} / 100) \cdot\left(1-\mathrm{s}^{\mathrm{e}}\right)$

= H/(2000+H) ; vgl. Wagner, F.H./Dirrigl, H. (1980), S. 169.

2) Vgl. Wagner, F.H./Dirrigl, H. (1980), S. $85 \mathrm{ff}$ 
(F.5) $\quad S^{v}=S^{v} \delta R$

(F.6) Sgk= sok $\delta R$.

Dividendenzahlungen sind Gewinnverwendung und mindern als solche die Bemessungsgrundlage der Ertragsteuern nicht. Ist BF der Betrag, der erforderlich ist, um die durch die Beteiligungsfinanzierung veranlaßten Auszahlungen (Dividenden, steuern) zu decken, beträgt die Gewerbeertragsteuerbelastung:

$$
S_{g e}=s_{g e}\left(B F-S_{g k}\right) .
$$

Im deutschen Steuersystem werden die Ausschüttungen schrittweise von der Körperschaftsteuer entlastet. Zunächst wird auf Gesellschaftsebene die körperschaftsteuerliche Belastung vom Normaltarif $\mathbf{s}^{\mathbf{k}}=0,56$ auf die Ausschüttungsbelastung von 36 prozent reduziert. Ist $D$ die mit der Ausschüttungsbelastung belastete Bardividende, beträgt die Entlastung bezogen auf $D$ $\mathrm{s}^{\mathrm{k}}=5 / 16$.

Die Körperschaftsteuerbelastung beträgt somit:

(F.8)

$$
S^{k}=s^{k n}\left(B F-S^{e}-S^{k}\right)-s^{k e D} .
$$

Die zweite stufe der Entlastung der Ausschüttungen von der Körperschaftsteuer erfolgt auf der Gesellschafterebene. Der persönlichen Einkommensteuer unterliegt die Bardividende zuzüglich des Anrechnungsanspruchs in Höhe der Ausschüttungsbelastung $\left(\mathbf{s}^{\mathrm{k} a}=9 / 16\right)$ :

$$
S^{e}=s^{e}\left(D+S^{k} a\right) .
$$

Außerdem unterliegt die Beteiligung der persönlichen Vermögensteuer.

In den bisherigen Ausführungen nicht berücksichtigt ist die Gesellschaftsteuer. Sie fallt als einmalige Zahlung bei der Kapitalerhöhung durch Ausgabe von Gesellschaftsrechten an ( $\$$ 2 I Ziff. 1 RVStG). 


\subsubsection{Selbstfinanzierung}

Die Selbstfinanzierung erfolgt aus versteuertem Gewinn. Da das selbstfinanzierte Kapital kein schuldposten im sinne des $\$ 103 I$ Bewg ist, beträgt der pro Geldeinheit des Bruttogewinns im Unternehmen zu Finanzierungszwecken zur Verfügung stehende Betrag:

$$
\begin{aligned}
& 1-s^{v}-s^{g}-s^{g} 0-s^{k} \\
& =\left(1-s^{k n}\right)\left(1-s^{g}\right)-s^{g k}\left(1-s^{k n}\right)\left(1-s^{g e}\right)-s^{v} .
\end{aligned}
$$

Zudem wird durch die steigerung des Anteilswerts auch die persönliche Vermögensteuer erhöht.

\subsection{Vergleich der Finanzierungsformen}

In diesem Abschnitt soll untersucht werden, ob ein Unternehmen gegenüber den jeweils paarweise zum Vergleich gestellten Finanzierungsformen indifferent ist, oder ob es eine der jeweiligen Finanzierungsalternativen präferiert.

\subsubsection{Fremdfinanzierung versus Beteiligungsfinanzierung}

Beim Vergleich der Fremd- und der Beteiligungsfinanzierung') können die Eigenschaften des deutschen steuersystems ausgenutzt werden, daß die Rapitaleinkommensarten zinsen und Dividenden derselben persönlichen Einkommensteuer sowie die als Fremd- oder Beteiligungskapital hingegebenen Finanzierungsmittel derselben persönlichen Vermögensteuer unterliegen. Somit muß in den Vergleich der beiden Finanzierungsformen aus der Kapitalgeber- bzw. Gesellschaftersphäre nur der Anrechnungsanspruch der Ausschüttungsbelastung der Dividenden ein-

1) Zum Folgenden vgl. Wagner, F.W./Dirrigl, H. (1980), S. $89 \mathrm{f}$ und Wöhe, G./Bilstein, J. (1986), S. $321 \mathrm{ff}$ sowie für die Kapitaleinkommensbesteuerung Sinn, H. - W. (1984), S. $234 f$ und derselbe (1985), S. $86 f$ 
gehen'). Zusätzlich wird vereinfachend angenommen, daß der Schuldposten FK und die entsprechende Erhöhung des Beteiligungskapitals $\delta R$ substanzsteuerlich gleich bewertet werden. Somit sind die folgenden Ertragsbedarfe miteinander zu vergleichen:

Für die Fremdfinanzierung:

$$
\begin{aligned}
F F & =Z I+S^{v}+S^{k}+S^{0}+S^{k} \\
& =Z I+s^{g} k D+\left[s^{g} \theta /\left(1-s^{g}\right)\right] \cdot D S Z I
\end{aligned}
$$

und für die Beteiligungsfinanzierung:

$$
\begin{aligned}
& B F=D+S^{v}+S^{g} k+S^{e}+S^{k} \\
& =\left[s^{v} /\left(1-s^{k n}\right)\left(1-s^{g} \theta\right)\right] \delta K+s^{g k} \delta K+\left(1-s^{g} \cdot\right)-1 \cdot D\left(1+s^{k a}\right)
\end{aligned}
$$

Die Fremdfinanzierung wird gegenüber der Beteiligungsfinanzierung präferiert, wenn gilt:

$$
\mathbf{F F}<\mathbf{B F}
$$

bzw.

$$
\begin{aligned}
Z I+\left[s^{g e} /\left(1-s^{g} \cdot\right)\right] D S Z I & +s^{g k} D S \\
<\left(1-s^{g e}\right)-1 \cdot D\left(1+s^{k a}\right) & +s^{g k} \delta K \\
& +\left[s^{v} /\left[\left(1-s^{k n}\right)\left(1-s^{g e}\right)\right]\right] \delta K .
\end{aligned}
$$

Werden zunächst die Ertragsteuern isoliert betrachtet, so besteht nur dann Indifferenz zwischen Fremd- und Beteiligungsfinanzierung, wenn die gesamten Fremdkapitalzinsen bei der Ermittlung des Gewerbeertrags als Dauerschuldzinsen hinzuzurechnen sind. Sobald diese Hinzurechnung nur teilweise ${ }^{2}$ ) oder

1) Diese Vorgehensweise impliziert, daß die Kapitalbedienungskosten, also zinseinkommen und Dividenden einschließlich des Anrechnungsanspruchs, gleich hoch sind: $\mathrm{ZI}=\left(1+\mathrm{s}^{\mathrm{ka}}\right) \mathrm{D}$.

2) In einer Ubergangsphase ab dem 1.1 .1983 erfolgte die Zurechnung $z u 60$ Prozent, seit dem 1.1.1984 erfolgt sie nur noch zu 50 Prozent. 
überhaupt nicht' ${ }^{1}$ erfolgt, besteht ertragsteuerlich eine eindeutige Präferenz für die Fremdfinanzierung.

In bezug auf die Belastung mit Gewerbekapitalsteuer gilt das für die Gewerbeertragsteuer Gesagte analog.

Als substanzsteuerliche Zusatzbelastung ist aus Gleichung (F.13) bei der Beteiligungsfinanzierung die ertragsteuerlich nicht absetzbare Vermōgensteuer ersichtlich. Sie beruht darauf, daß Kapitalgesellschaften selbständig steuerpflichtig sind und das Beteiligungskapital im Gegensatz zum Fremakapital nicht als schuldposten bei der Vermögensaufstellung abzugsfähig ist.

Somit sind die steuerlichen Regelungen Grund für eine eindeutige Präferierung der Fremdfinanzierung ${ }^{2}$ ). Zum einen führt die nur teilweise Hinzurechnung der Dauerschulden bzw. Dauerschuldzinsen bei der Gewerbesteuer zum Gewerbekapital bzw. Gewerbeertrag zu einer Diskriminierung der Beteiligungsfinanzierung ${ }^{3}$ ). Als zweites verursacht die eigenständige Vermögensteuerpflicht der Kapitalgesellschaften eine einseitige Belastung des Beteiligungskapitals.

Zusätzlich führt die bereits angesprochene Gesellschaftsteuer zu einer weiteren Schlechterstellung der Beteiligungsfinanzierung. Sie fällt bei einer Kapitalerhöhung durch Beteiligungsfinanzierung an, da mit ihr der Erwerb von Gesellschaftsrechten verbunden ist (\$ 2 I Ziff. 1 KVStG).

1) Bei kurzfristigem Fremdkapital erfolgt keine Hinzurechnung. Allerdings stellt sich die hier diskutierte Alternative dann in der Regel nicht.

2) Vgl. Knobbe-Keuk, B. (1987), S. 131

3) Wenngleich systemwidrig (vgl. Littmann, K. (1980), S. 620f), so wahrte die vollständige Hinzurechnung sowohl der Dauerschulden als auch der Dauerschuldzinsen den Charakter der Gewerbesteuer als einer objektsteuer zumindest in bezug auf ihre Unabhangigkeit von der Kapitalstruktur der Unternehmung. 


\subsubsection{Selbstfinanzierung versus Beteiligungsfinanzierung}

Diese Paarstellung', impliziert, daß die Frage nach der Rapitalverwendung zugunsten einer Anlage in der Rapitalgesellschaft bereits beantwortet ist. $\mathrm{Zu}$ analysieren ist hier, durch welche Finanzierungsform der im Unternehmen verfügbare Betrag maximiert wird. Da die Gewerbeertrag- und substanzsteuerbelastung bei beiden Finanzierungsarten gleich ist, brauchen die entsprechenden steuern nicht in den Kalkül mit einbezogen $z u$ werden.

Der bei der Selbstfinanzierung im Unternehmen verfügbare Betrag (SE) ist der mit Vermögen- und Gewerbesteuer belastete Gewinn (G), verringert um die bei Thesaurierung fällige Rörperschaftsteuer:

(F.14) $\quad S E=\left(1-s^{k n}\right) G$.

Bei der Beteiligungsfinanzierung fallen neben der Gesellschaftsteuer (SoB) weitere Emissionskosten (EM) an. Beide Ausgabenarten sind ertragsteuerlich absetzbar. Der nach Abzug der persönlichen Einkommensteuer einlagefähige Betrag beläuft sich somit auf:

$$
B E=G-S^{e}-\left(S^{g} s+E M\right)\left(1-s^{k n}\right)\left(1-s^{g} e\right) .
$$

Bei einer proportionalen Einkommensbesteuerung wäre die Problemstellung trivial. Die Selbstfinanzierung ist dann vorzuziehen, wenn gilt:

$$
s^{k n} s^{e}+\left(1-s^{e}\right)\left[\left(s^{g e s}+e m\right)\left(1-s^{k n}\right)\left(1-s^{g e}\right)\right] \text {, }
$$

d.h. der persönliche steuersatz zuzüglich der Nettobelastung mit Emissionskosten pro Geldeinheit nach Einkommensteuer müßte den körperschaftsteuerlichen Thesaurierungssatz übersteigen.

1) Vgl. Wagner, F.W./Dirrigl, H. (1980), S. 137 und Wöhe, G./Bilstein, J. (1986), S. $327 f f$ sowie für die Kapitaleinkommensbesteuerung Sinn, H. -W. (1984), S. $233 f$ und derselbe (1985), S. $81 f$ 
Ein Planungsproblem ergibt sich erst bei progressiver Einkommensbesteuerung. Ausschüttung und Wiedereinlage sind dann solange zu präferieren, wie der pro ausgeschütteter Geldeinheit wiedereinlagefähige Betrag größer als der Thesaurierungsbetrag ist. Bezeichnet $\mathbf{s}^{{ }^{\prime}}$ die Marginalbelastung mit Einkommensteuer, so muß gelten:

$$
\left(1-s^{e}\right)\left(1-\left[s^{g} \operatorname{s}+e m\right]\left[\left(1-s^{k}\right)\left(1-s^{0} \cdot\right)\right]\right) \leq 1-s^{k n} .
$$

Fallen keine nichtsteuerlichen Emissionskosten an und wird ein Hebesatz der Gewerbesteuer von 400 Prozent unterstellt, so beträgt der kritische Grenzsteuersatz der Einkommensteuer bei dem im Jahr 1987 geltenden Tarif 55,838 Prozent, der einem zu versteuernden Einkommen von 128.650 DM entspricht. Wird in den persönlichen Einkommensteuersatz die Rirchensteuer $\left(s^{k} 1=0,09\right)$ einbezogen und betragen die nichtsteuerlichen Emissionskosten em $=0,05$, dann ist der kritische Einkommensteuersatz $\mathrm{s}^{{ }^{\prime}}=52,869$ Prozent schon bei 103.910 DM erreicht').

\subsubsection{Selbstfinanzierung versus Fremdfinanzierung}

Wird angenommen, daß die Sollzinsen nicht kleiner als die Habenzinsen sein werden, dann ist die hier untersuchte Fragestellung nur sinnvoll, wenn wie im vorangegangenen Abschnitt bereits eine Entscheidung zugunsten einer Anlage der Mittel im Unternehmen gefallen ist. Es soll also untersucht werden, ob Thesaurierung oder die Gewährung eines Gesellschafterdarlehens die für das Unternehmen in bezug auf den Verfügungsbetrag vorteilhaftere Finanzierungsform darstellen. Sind der Marginalsatz der Einkommensteuer und der Körperschaftsteuersatz gleich hoch, so ist nach der Analyse des letzten Abschnitts unmittelbar einsichtig, daß die selbstfinanzierung die Fremdfinanzierung nie dominiert. Werden zusätzlich die eigenständige Vermōgensteuerpflicht der Kapitalgesellschaft sowie die unvollständige Hinzurechnung von Dauerschulden und

1) Zu den Auswirkungen der Steuerreform 1990 auf das Schütt-ausHol-zurück-Verfahren vgl. Kapitel H 
Dauerschuldzinsen bei der Ermittlung der Gewerbesteuer berücksichtigt, wird die Fremdfinanzierung gegenüber der Selbstfinanzierung eindeutig präferiert' '.

Ein Planungsproblem entsteht, wenn die steuerlichen Kosten der Thesaurierung pro DM Gewinn unter dem spitzensteuersatz der Einkommensteuer liegen. Dann ist Ausschüttung und anschließende Darlehensgewährung solange günstiger als die Selbstfinanzierung durch Einbehaltung der Gewinne, wie der persönliche Grenzsteuersatz der Einkommensteuer kleiner ist als die pro Einheit der thesaurierten Gewinne anfallenden steuern. Die Kosten der Thesaurierung setzen sich zusammen aus dem Körperschaftsteuersatz auf die einbehaltenen Gewinne zuzüglich des Barwerts des Vermögensteuersatzes über den Planungshorizont sowie des Barwerts der gewerbesteuerlichen Privilegierung des Fremdkapitals pro thesaurierter Geldeinheit.

\subsection{Der Einfluß der Finanzierungsbedingungen auf die In- vestitionsplanung}

Der Vergleich der Finanzierungsformen zeigt, daß bei gleicher Kapitalbedienung $\left(\mathrm{ZI}=\left[1+\mathbf{s k}^{\mathrm{a}}\right] \mathrm{D}\right)$ die Fremdfinanzierung der Beteiligungs- und der Selbstfinanzierung unter dem geltenden steuerrecht immer überlegen ist. Werden die Ergebnisse dieses Abschnitts in bezug auf die Investitionsplanung im oben dargestellten neoklassischen Investitionsmodell betrachtet, so zeigt sich, daß auch bei optimaler Finanzierung, d.h. Fremdfinanzierung, die marginale Investitionsbereitschaft durch die Besteuerung negativ beeinflußt wird. Grund dafür ist, daß aufgrund der Gewerbesteuer, die keine Ziel- (Gewinn-) steuer ist, nicht die gesamten Opportunitätskosten (hier: Zurechnung der Dauerschuldzinsen) des Kapitalbestands steuerlich abge-

1) In diesem Zusammenhang ist auch zu beachten, daß Zinssubventionen, die die Kreditkosten und den Ertrag von Alternativanlagen außerhalb des Unternehmens senken, $z u$ einer Substitution von Eigenkapital durch Fremdkapital führen können. Dies ist auch die einzige Entscheidungssituation, in der sich für eine Personengesellschaft ein Entscheidungsproblex zwischen der Aufnahme von Fremdkapital und der Eigenfinanzierung stellt. 
setzt werden können und deshalb die Marginalinvestition steuerlich belastet wird.

Auch ohne diese ertragsteuerliche Verzerrung würde die Investitionsbereitschaft durch die Besteuerung negativ beeinflußt, da auch die steuerliche Belastung des Kapitalbestandes einen Reil zwischen die Grenzproduktivität und die opportunitätskosten des Kapitaleinsatzes treibt' ${ }^{\prime}$.

\subsection{Fazit}

Die Untersuchung der steuerlichen Behandlung alternativer Finanzierungsformen ergibt eine eindeutige Präferenz für die Fremdinanzierung. Diese wurde durch die zulassung eines begrenzten Abzugs der Dauerschuldzinsen und der Dauerschulden von den Bemessungsgrundlagen der Gewerbesteuer verstărkt. Die Gründe dafür, daß die verschiedenen Formen der Kapitalbeschaffung steuerlich nicht neutral behandelt werden, sind neben der Struktur der Gewerbesteuer die Substanzsteuern sowie die Gesellschaftsteuer. Die steuerliche Behandlung der alternativen Finanzierungsformen kann somit als ein Faktor zur Erklärung des empirisch beobachtbaren Rückgangs der Eigenkapitalquoten herangezogen werden'). Aber auch die steuerliche Behandlung der Fremdfinanzierung verursacht Verzerrungen im Investitionskalkül und führt $z u$ einer Dämpfung der Investitions- und damit auch der Innovationsbereitschaft. Verantwortlich dafür ist die Tatsache, daß bei der Fremdfinanzierung nicht die gesamten opportunitätskosten des Kapitaleinsatzes steuerlich abgesetzt werden können. Hinter der nun folgenden Diskussion der Finanzierung von Innovationsprojekten steht die Frage, ob Innovationsaktivitäten stärker als andere Investitionsvorhaben durch die steuerliche Behandlung der Rapitalbeschaffung gedämpft werden. Dies wäre dann der Fall, wenn Innovatoren im Vergleich $z u$ anderen Investoren ihre Vorhaben verstärkt mit Eigenkapital, das gegenüber dem Fremdkapital diskriminiert wird, finanzieren müßten.

1) Vgl. Sinn, H.-W. (1985), S. 131

2) Vgl. Sinn, H.-W. (1985), S. $92 \mathrm{ff}$ 


\section{$\underline{2.1}$}

Problemstellung

In der startphase eines Innovationsprojekts stehen den Aufwendungen keine oder nur relativ geringe Erlöse gegenüber. Es besteht somit ein andauernder Kapitalbedarf bis die Einnahmen die Ausgaben übersteigen. Selbstfinanzierung ist in der Regel nur sehr begrenzt möglich. Der Kapitalbedarf übersteigt insbesondere bei kleinen und mittleren Unternehmen die finanziellen Mittel, die aus dem Privatvermögen oder durch Thesaurierung von Gewinnen in anderen Unternehmensbereichen aufgebracht werden können.

Für das innovationsbereite Unternehmen stellt sich somit das Problem der Beschaffung von externen Finanzierungsmitteln. Anlagebereites Rapital von außerhalb des Unternehmens muß akquiriert werden. Gerade bei Innovationen handelt es sich um das Finanzierungsproblem schlechthin'1). Der Unternehmer benötigt finanzielle Mittel, um eine von ihm als gewinnträchtig eingeschätzte Innovationsmöglichkeit zu nutzen. Um sein riskantes, damit aber auch chancenreiches Vorhaben zu realisieren, muß ihm das Kapital längerfristig und ohne die Gefahr des Abzugs zur Verfügung stehen.

Dagegen stehen die Interessen der potentiellen Kapitalgeber. Sie wünschen wenig riskante Anlagen und wollen nur kleine Beträge investieren, um Diversifikationsmöglichkeiten zu behalten. Zudem sind sie an tendenziell kürzerfristigen Engagements interessiert, um über ihr Kapital auch kurzfristig verfügen zu können.

Neben den bei der Rapitalanlage bereits offenkundig widerstreitenden Interessen bezüglich Losgröße (Finanzierungsvolumen), Fristen und Risiken treten nach der Gewährung des Kapitals zusätzliche Anreiz- und Informationsprobleme auf, da der Rapitalnehmer Entscheidungen treffen kann, die die Rapitalgeber belasten. Je jünger das Unternehmen und je neuartiger das Innovationsprojekt, desto größer sind die Informationsprobleme für die Kapitalanleger und die Entscheidungsspielräume der Kapitalnehmer.

1) Vgl. Schmidt, R.H. (1985), S. $426 f f$ 
Sind Kapitalgeber und Rapitalnehmer auf direkte Finanzbeziehungen angewiesen, können sie nur nach hohen suchkosten zu einem Interessenausgleich in komplexen Verträgen kommen. Es haben sich jedoch Institutionen herausgebildet, die eine Transformationsfunktion zum Ausgleich von Fristen-, Risiko-, Losgrößen- und Informationsinteressen übernehmen. Diese Vermittlungsfunktion wird von Finanzintermediären, also Banken und organisierten Finanzmärkten (Börsen) wahrgenommen. Im folgenden werden die Möglichkeiten der Fremdfinanzierung von Innovationsprojekten untersucht. Im Anschluß daran werden dann die Bedingungen der Beschaffung von Eigenkapital für risikobehaftete Projekte diskutiert.

\subsection{Fremdfinanzierung}

\subsubsection{Fremdkapitalaufnahme bei Rreditinstituten}

Die Rreditvergabeentscheidung der Banken erfolgt als Einzelentscheidung. Der jeweilige Entscheidungsträger hat keinen Oberblick über die Gesamtheit der bereits. getroffenen Vergabeentscheidungen und über die Gesamtheit der eingegangenen Kreditanträge. Seine Informationsgrundlagen bilden neben Analysen der gesamtwirtschaftlichen und branchenspezifischen Entwicklung die Jahresabschlüsse, Geschäftsberichte und Finanzplāne der Unternehmen, sowie die Unterlagen über die Inanspruchnahme sonstiger Bankleistungen und seine Einschätzung der Managementfähigkeiten der Unternehmensleitung.

Damit gehen Höhe und struktur der gesamten an das Institut gerichteten Kreditnachfrage und insbesondere der Grenzeffekt, den der betrachtete Rreditantrag auf die Risikoposition der Bank ausübt, nicht in die Kreditvergabeentscheidung ein. Dies hat zur Folge, daß Risikostreuungs- und Diversifikationsaspekte bei der Entscheidung unberücksichtigt bleiben.

Wird den Banken ein risikoaverses Verhalten unterstellt, so bedeutet dies, daß bei ihren Entscheidungen die möglichen ungünstigen Ereignisse betont werden. Aus den beschriebenen institutionellen Bedingungen der Entscheidungsfindung und der Risikoeinstellung der Entscheidungsträger lassen sich folgen- 
de Aussagen über das Kreditvergabeverhalten der Banken ablei$\operatorname{ten}^{1}$ ):

1. Kreditentscheidungen sind dann optimal, wenn die Insolvenzwahrscheinlichkeit der Bank minimiert wird.

2. Optimale Kreditentscheidungen maximieren das bei ungünstigem Ausgang resultierende Eigenkapital.

Diese Schlußfolgerungen lassen sich aus einer einfachen Nutzenfunktion der Form

$$
U=-e^{-\alpha R}
$$

ableiten, wobei $\alpha>0$ das Maß für die Risikoaversion darstellt und $R$ das Eigenkapital repräsentiert.

Wird jetzt $U$ als eine Funktion der Risikoaversion $\alpha$ interpretiert, so ist Gleichung (F.18) gleich der negativen LaplaceTransformation der Wahrscheinlichkeitsverteilung der Eigenkapitalbestände der Bank dF(R):

$$
\begin{aligned}
U(\alpha) & =-E\left(e^{-\alpha R}\right) \\
& =-\int_{R=0}^{\infty} e^{-\alpha R} d F(R) .
\end{aligned}
$$

Für den Grenzfall unendlich großer Risikoaversion gilt2 ):

(F.20) $\lim \left[-\int_{R=0}^{\infty} \mathrm{e}^{-\alpha R} \mathrm{dF}(R)\right]=W K(R=0) ;$

bei extremer Risikoaversion geht Gleichung (F.20) gegen den Wert der Wahrscheinlichkeit, daß das Eigenkapital der Bank null wird, also durch Verluste völlig aufgezehrt wird. Bezogen auf die präferenzfunktion (F.18) heißt dies jedoch, daß die Wahrscheinlichkeit des Eigenkapitalverlusts zu minimieren ist, womit Aussage 1 nachgewiesen ist.

Aussage 2 kann analog über die Betrachtung des zur Nutzenfunktion (F.18) gehörenden Sicherheitsäquivalents bewiesen werden ${ }^{3}$ ) :

1) Vgl. Wilhelm, J. (1982), S. 21

2) Vgl. Allen, R.G.D. (1971), S. $187 \mathrm{ff}$ und Feller, W. (1971), S. $429 \mathrm{f}$

3) Vgl. Bamberg, G./Spremann, K. (1981), S. $208 f$ 
(F.21) $S_{\alpha}=-\alpha^{-1} \ln \left[E^{-\alpha R}\right]$.

R sei der kleinstmögliche Wert, den das Eigenkapital in der Verteilungsfunktion $\mathrm{dF}(R)$ annehmen kann. Gleichung (F.21) kann umgeformt werden zu:

(F.22) $\quad S_{\alpha}=\bar{R}-\alpha^{-1} \ln \left[\int_{R=\bar{R}}^{\infty}(-\alpha[R-\bar{R}]) d F(R)\right]$,

analog zu (F.20) gilt1):

(F.23) $\quad \lim _{\alpha \rightarrow \infty} S_{\alpha}=\bar{R}$;

im Grenzfall unendlich großer Risikoaversion konvergiert das Sicherheitsäquivalent gegen den Eigenkapitalbestand, der im ungünstigsten Fall realisiert wird. Bezogen auf die Nutzenfunktion ist somit auch die zweite Aussage belegt. Das Sicherheitsäquivalent konvergiert bei unendlich großer Risikoaversion gegen den Minimalwert $\bar{R}$, den die Verteilungsfunktion annehmen kann.

Die Konsequenzen eines solchen Verhaltens der Banken für das einzelne Unternehmen können abgeleitet werden, indem das sicherheitsäquivalent für einen marginalen kredit formuliert wird.

In bezug auf den marginalen Rredit gelte:

$$
\overline{\mathbf{R}}=0,
$$

d.h. durch die zusätzliche Rreditgewährung darf die Eigenkapitalposition bei Eintreten des ungünstigsten Falles nicht geschmälert werden.

Der Grenzeffekt $\delta R$ des zusätzlichen Rredits A auf die Eigenkapitalposition der Bank wird durch die Geldbeschaffungskosten $r$, den Rapitalkostensatz $i$, der dem Schuldner berechnet wird, und die Befriedigungsquote $y$ bestimmt ${ }^{2}$ :

$(F .24) \quad \delta R=A[(1+i) Y-(1+r)]$

1) Vgl. Bamberg, G./Spremann, K. (1981), S. $208 \mathrm{f}$

2) Vgl. Wilhelm, J. (1982), S. 22 
bzw. eingesetzt in Gleichung (F.23) für den ungünstigsten Fall:

$$
S_{-}=S\{(\mathbb{A}[(1+i) y-(1+r)]\}=\bar{R} \text {. }
$$

Für $\bar{R}=0$ bedeutet dies, daß der Rreditbetrag, den das Unternehmen maximal erhalten kann, auf die summe begrenzt ist, die es im ungünstigsten Fall einschließlich der Geldbeschaffungskosten mit sicherheit der Bank zurückzahlen kann. Für $\bar{R}=0$ ist die Bank also nur im Bereich positiver Deckungsbeiträge bereit, Risiken einzugehen.

Das in diesem Ansatz unterstellte Bankenverhalten wird durch empirisches Datenmaterial bestätigt. Die Frage, ob Banken bereit sind, ihre Rreditbereitschaft bei entsprechender Vergütung des Risikos zu erhöhen, kann nicht direkt untersucht werden, da über Kreditbeziehungen Diskretion gewahrt wird. Die Analyse der Forderungsausfälle gibt jedoch indirekt Aufschluß darüber, daß die Banken bei ihren Kreditvergabeentscheidungen das sicherheitsziel betonen und Rreditrisiken zu beschränken trachten. So berichten Friend/Howitt von einer Ausfaliquote im kanadischen Bankwesen von etwa 0,5 Prozentbezogen auf das Rreditportefeuille, wobei im betrachteten Zeitraum von zehn Jahren keine Bank einen höheren wertmäßigen Kreditausfall als 0,7 Prozent erlitt'). In der Blisdesrepublik Deutschland beträgt das Volumen der Wertberichtigungeis bezogen auf den Rreditbestand gegenüber inländischen Nichtbanken 0,3 Prozent'2).

Trotz dieses risikoaversen Rreditvergabeverhaltens können Kreditausfälle auftreten. Grund dafür sind Fehleinschätzungen der identifizierten Risiken bzw. die Nichtberücksichtigung einzelner Risiken. Da vollkommene Information nicht zu erreichen ist, werden derartige latente Risiken immer bestehen bleiben'). Weitere Risiken bei der Rreditvergabe resultieren aus den Interessenkonflikten zwischen Rapitalgebern und Rapitalnehmern'). Der Kapitalnehmer hat ein Interesse an der Be-

1) Vgl. Friend, J./Howitt, P. (1980), S. 472

2) Vgl. Deutsche Bundesbank, Monatsbericht Februar 1988, S. $10 \mathrm{ff}$

3) Vgl. Wilhelm, J. (1982), S. $24 \mathrm{f}$

4) Vgl. Schmidt, R.H. (1981), S. $140 \mathrm{ff}$ 
schaffung von Finanzierungsmitteln für die von ihm als vorteilhaft angesehene Investition. In seinen Informationen an den Kapitalgeber wird er, um die Mittel zu erhalten, dieses Projekt im Zweifel günstiger darstellen als er es selbst bewertet. Zudem muß sich der Rreditgeber dagegen schützen, daß nach dem zustandekommen der Kreditbeziehung für den Kapitalnehmer andere Entscheidungen optimal werden. So kann der Kreditnehmer seine Investitionspolitik so ändern, daß sein individueller Nutzen zu Lasten des Kapitalgebers in Form von fringe benefits oder riskanteren Investitionsstrategien erhöht wird'). Der Kapitalgeber muß deshalb Rosten zur Uberwachung und sicherung seiner Interessen eingehen ${ }^{2}$ ).

Die Bewertung dieser latenten Risiken sowie die Beurteilung der Qualität der Informationen und Sicherheiten, die der Kreditnehmer bereitstellt, verursachen die trotz Kreditbeschränkung $z u$ beobachtende zinssatzdifferenzierung ${ }^{3}$ ).

Wie bereits mehrfach angesprochen sind gerade Innovationen mit sehr hohen und nur schwer bewertbaren Risiken verbunden, zumal auch nicht auf Erfahrungswerte zurückgegriffen werden kann. Wie aus der Analyse der Rreditvergabeentscheidung der Banken hervorgeht, können unter diesen Bedingungen Finanzierungsmittel nur erhalten werden, wenn sicherheiten gestellt werden. Diese stehen aber insbesondere neuen sowie auch kleinen und mittleren Unternehmen regelmäßig nicht bzw. nur unzureichend zur Verfügung. Somit ist für innovationsbereite Unternehmen der Weg der Fremdmittelbeschaffung über Banken nur sehr begrenzt begehbar. Vor allem in der Entwicklungsphase ist dieser Weg praktisch versperrt. "Der Wert der Entwicklungen steckt(e) ja nur in den Kosten, nicht aber in den Aktiva der Bilanzen." 4 ).

1) Vgl. Schmidt, R.H. (1985), S. 427

2) Vgl. zum Bereich der Agency Costs den Oberblick bei Zechner, J. (1982), S. 182ff sowie die dort angegebene Literatur, insbesondere Akerlof, G.A. (1976), S. $488 \mathrm{ff}$ und Jensen, M.C./Meckling, W.H. (1976), S. $305 \mathrm{ff}$

3) Vgl. Wilhelm, J. (1982), S. $26 \mathrm{ff}$

4) Lettenmayer, S. (1983), S. 56 
2.2.2 Fremdfinanzierung mit Schuldscheindarlehen und Industrieobligationen

Schuldscheindarlehen sind "anleiheähnliche, langfristige Großkredite"1), die in der Regel von Versicherungsunternehmen, insbesondere Lebensversicherungen und Pensionskassen der öffentlichen Hand und privaten Unternehmen gewährt werden. Versicherungen unterliegen dem Versicherungsaufsichts-Gesetz (VAG) und sind dadurch in ihrer Anlagepolitik vor allem durch die $\$ \S 54 f$ VAG beschränkt. Für die Darlehen an Unternehmen wird in $\$ 54 \mathrm{a}$ II ziff. 8d VAG verlangt, daß "auf Grund der bisherigen und der zu erwartenden künftigen Entwicklung der Ertrags- und Vermögenslage des Unternehmens die vertraglich vereinbarte Verzinsung und Rückzahlung gewährleistet erscheint" und zudem die Darlehen erstrangig gesichert sind. Aufgrund dieser Anforderungen sind neue sowie auch kleine und mittlere Unternehmen, deren Unternehmensrisiko durch die zu finanzierende Innovation bestimmt wird, nicht "schuldscheinfähig".

Obligationen sind festverzinsliche und börsengãngige Teilschuldverschreibungen, die auf einen bestimmten Nennbetrag lauten. Da auch für diese Finanzierung in erster Linie institutionelle Anleger als Kreditgeber in Frage kommen, werden auch an die Obligationen die Anforderungen der Deckungsstockfähigkeit des VAG gestellt. Zudem ist für die Emission einer Anleihe eine staatliche Genehmigung erforderlich ( $\$ 795 f f$ BGB). Neben der hier erfolgenden Bonitätsprüfung ist bis zur Börseneinführung noch ein Verfahren beim Zentralen Rapitalmarktausschuß sowie ein positiver Bescheid der Börsenzulassungsstelle (\$ 36 BörsG) erforderlich. Auf die Anforderungen für die Börsenzulassung soll bei der Diskussion der Beschaffung von Eigenkapital über den Kapitalmarkt näher eingegangen werden. Für die Anleihefinanzierung kann aber festgestellt werden, daß die Anforderungen an die kapitalsuchenden Unternehmen gegenüber der Finanzierung über schuldscheindarlehen weiter erhöht werden und daß somit dieser Finanzierungsweg praktisch nur großen, eingeführten Unternehmen offen steht ${ }^{2}$ ).

1) Drukarczyk, J. (1985), S. 201 und Reinboth, H. (1976), Sp. 1594

2) Vgl. Drukarczyk, J. (1985), S. $203 f f$ 


\subsubsection{Finanzierung durch Pensionsrückstellungen}

Unternehmen können während deren Betriebszugehörigkeit für ihre Arbeitnehmer Pensionsrückstellungen bilden, um ihnen eine betriebliche Altersversorgung zu gewähren. Die periodischen Zuführungen zu diesen Rückstellungen sind ertragsteuerlich als Aufwand abzugsfähig (\$ 6a EStG); substanzsteuerlich werden die Pensionsrückstellungen wie Fremdkapital behandelt ( $\$ 104$ BewG). Den periodischen zuführungen zu den Rückstellungen stehen erst bei Eintritt des Versorgungsfalles Auszahlungen gegenüber. Somit sind für die zeit der Betriebszugehörigkeit des Arbeitnehmers liquide Mittel im Unternehmen verfügbar, soweit in Höhe der Rückstellungen Gewinne erzielt wurden. Werden die Pensionsrückstellungen unter dem Finanzierungsaspekt betrachtet, dann sind sie an die gleichen Voraussetzungen gebunden wie die Eigenfinanzierung durch Thesaurierung oder das Schütt-aus-Hol-zurück-Verfahren. Der Kapitalbedarf tritt zudem bei innovierenden Unternehmen vor allem dann auf, wenn hohen Aufwendungen vergleichsweise geringe Erträge gegenüber stehen. Die Finanzierung über Pensionsrückstellungen ist somit insbesondere für junge, kleine und mittlere unternehmen keine realistische Alternative.

\section{$\underline{2.2 .4 \quad \text { Zusammenfassung }}$}

Die Analyse der Möglichkeiten der Fremdkapitalbeschaffung zeigt, daß kleine und mittlere Unternehmen nur begrenzt Fremdmittel beschaffen können. Dies betrifft insbesondere neugegründete Unternehmen, da diese nicht am Markt eingeführt sind, über sie deshalb nur wenige Informationen vorliegen und sie dadurch für die Rapitalgeber mit erhöhten Risiken verbunden sind.

Die Finanzierung über Schuldscheindarlehen und Anleihen scheidet in der Regel aufgrund der institutionellen Anforderungen aus. Die Möglichkeit der Finanzierung über langfristige Bankkredite ist wegen des Charakters der zu finanzierenden Investition und des risikoaversen Rreditvergabeverhaltens der Banken eng begrenzt. 
Innovationswillige Unternehmen müssen deshalb im allgemeinen Möglichkeiten suchen, haftendes und damit voll risikotragendes Eigenkapital zu beschaffen.

\section{$\underline{2.3 \quad \text { Eigenfinanzierung }}$}

\subsubsection{Eigenfinanzierung und Rechtsform}

Bevor die Möglichkeiten der Beschaffung von Eigenkapital näher diskutiert werden, sollen die sich aus der Rechtsform der Unternehmen ergebenden Bedingungen der Eigenfinanzierung skizziert werden. Die Darstellung der Beteiligungsformen erfolgt aus der Sicht des Rapitalanlegers. Seine Anlageentscheidung richtet sich nach dem Risiko, das mit dem Engagement verbunden ist, und nach der Fristigkeit der Beteiligung. Die Ausführungen $z u$ den Formen der Eigenfinanzierung konzentrieren sich deshalb auf die rechtliche Ausgestaltung des Gesellschaftsvermögens und die aus der Anlage resultierenden Haftungsrisiken, auf die Vermögens- und Kontrollrechte, die dem Kapitalgeber zustehen, sowie auf die Möglichkeiten, sein Engagement zu beenden.

Das Einzelunternehmen ist keine Gesellschaft im Sinne eines Zusammenschlusses mehrerer zur Verfolgung eines gemeinsamen Zwecks'). Es besitzt somit auch kein Gesellschaftsvermögen. Das Einzelunternehmen ist vielmehr Teil des Gesamtvermögens des Eigentümers ${ }^{2}$ ). Somit sind die Eigenfinanzierungsmöglichkeiten durch die Höhe des Privatvermögens begrenzt. Eine Erhöhung des Eigenkapitals über das Privatvermögens hinaus ist nur durch Bildung einer Gesellschaft möglich.

stiller Gesellschafter ist, wer sich mit einer Einlage am Handelsgewerbe eines anderen beteiligt; die Einlage geht dabei in das Vermögen des Inhabers über ( $\$ 335$ I HGB). Der stil-

1) Vgl. Hueck, G. (1983), S. 1

2) Vgl. Drukarczyk, J. (1985), S. 112 
le Gesellschafter ist am Gewinn beteiligt, er kann auch am verlust beteiligt sein (\$ 336 HGB). Sein gesetzlich garantiertes Rontrollrecht beschränkt sich darauf, eine "abschriftliche Mitteilung der jährlichen Bilanz zu verlangen und ihre Richtigkeit unter Einsicht der Papiere und Bũcher zu prüfen" ( $\$ 338 I$ HGB).

Der stille Gesellschafter kann seine Einlage mit einer Frist von 6 Monaten zum Schluß des Geschäftsjahres kündigen $(\$ 339$ HGB). Je nach Vertragsgestaltung hat er auch Anspruch auf einen Anteil am Unternehmenswertzuwachs.

Im Ronkursfall kann der stille Gesellschafter seine Einlage, soweit sie nicht durch Verluste aufgezehrt ist, als Forderung geltend machen ( $\$ 341$ I HGB).

Eine offene Handelsgesellschaft (OHG) ist eine zum Betrieb eines Handelsgewerbes unter gemeinschaftlicher Firma errichtete Personengesellschaft mit unbeschränkter Haftung aller Gesellschafter ( $\$ 105$ I HGB).

Alle Gesellschafter sind geschäftsführungsbefugt (\$ $114 I$ HGB). Auch von der Geschäftsführung ausgeschlossene Gesellschafter können sich durch Einsicht in die Bücher persönlich unterrichten (\$ $118 \mathrm{I}$ HGB). Die Gesellschafter haben ein Kündigungsrecht ( $\$ 131$ ziff. 6 HGB) und Anspruch auf einen Anteil am Unternehmenswert.

An einer Rommanditgesellschaft (RG) sind neben wie bei der OHG voll haftenden Gesellschaftern (Romplementären) auch beschränkt haftende Gesellschafter (Rommanditisten) beteiligt (\$ 161 I HGB).

Die Kommanditisten sind von der Geschäftsführung ausgeschlossen und können nur bei außerordentlichen Geschäften den geschäftsführenden Komplementären widersprechen ( 164 HGB). Ihre Rontrollrechte entsprechen denen eines stillen Gesellschafters (\$ $166 \mathrm{HGB}$ ). Ein Rommanditist kann seinen Anteil wie ein OHG-Gesellschafter kündigen (\$ 161II HGB).

Die Gesellschaft mit beschränkter Haftung (GmbH) ${ }^{1}$ ) ist eine juristische Person. Die Haftung der Gesellschafter ist auf

1) Zum Folgenden vgl. Kübler, F. (1981), S. $218 \mathrm{ff}$ 
ihre stammeinlagen beschränkt'). Das Mindest-stammkapital beträgt 50.000 DM und ist ausschüttungsgesperrt. Die Gesellschafter haben Anspruch auf einen Anteil am Gewinn und/oder Liquidationserlös ( $\$ \$ 5,13,26 f f, 30$ GmbHG). Die Geschäftsführer werden von der Gesellschafterversammlung bestellt und können selbst auch Gesellschafter sein (\$ 46 Ziff. 5 GmbHG). Jeder Gesellschafter hat Anspruch auf Auskunft und Einsicht in die Geschäftsbücher ( $\$ 51$ a GmbHG) sowie das Recht zur Anfechtung der Geselischafterbeschlüsse ${ }^{2}$ ).

Die obertragung von Geschätsanteilen bedarf der notariellen Beurkundung ( $\$ 15 \mathrm{GmbHG}$ ). Zudem kann ein Gesellschafter aus wichtigem Grund eine Auflösungsklage erheben ( $\$ 61$ GmbHG).

Wie die GmbH ist auch die Aktiengesellschaft (AG) eine juristische Person. Für die gegenüber der AG bestehenden Forderungen haftet den Gläubigern nur das Gesellschaftsvermögen. Sein in Aktien zerlegtes Mindest-Nennkapital belâut sich auf 100.000 DM (\$ 1 AktG). Die Haftung der Aktionäre beschränkt sich auf die beim Erwerb der Aktie geleistete Einlage; sie darf weder verzinst noch zurückbezahlt werden ( $\$ \$ 54,57$ AktG).

Bei der AG erfolgt eine Trennung zwischen Eigentum und Verfügungsmacht. Die Geschäftsführung wird von einem eigenverantwortlich handelnden Vorstand übernommen. Die Vorstandmitglieder haben die Geschäte mit der sorgfalt eines ordentlichen und gewissenhaften Geschäftsleiters zu führen. Sie werden vom Aufsichtsrat bestellt und sind ihm gegenüber berichtspflichtig ( $\$ \S 76,84,90,93 \mathrm{AktG}$ ). Der Aufsichtsrat überwacht die Geschäftsführung und kann bestimmte Arten von Geschäften von seiner Zustimmung abhängig machen ( $\$ 111$ AktG).

Die Aktionäre können ihre Rechte in der Hauptversammlung ausüben'). Sie bestellen die Mitglieder des Aufsichtsrats soweit diese nicht nach mitbestimmungsrechtlichen Vorschriften in den Aufsichtsrat entsandt oder gewählt werden müssen. Zudem

1) Allerdings kann im Gesellschaftsvertrag eine Nachschußpflicht vereinbart werden.

2) Vgl. Rübler, F. (1981), S. 231

3) Das stimmrecht kann ausgeschlossen werden, wenn ein Vorzug bei der Gewinnverteilung gewăhrt wird (\$ 12, 139ff AktG). 
entscheiden sie über die Entlastung der Mitglieder des Vorstands und des Aufsichtsrats, die Bestellung der Abschlußund Sonderprüfer, über Satzungsänderungen und Maßnahmen der Rapitalbeschaffung und Rapitalherabsetzung sowie über die Verwendung des Bilanzgewinns. Dieser wird zuvor von Vorstand und Aufsichtsrat festgestellt. Zudem besitzt jeder Aktionär ein Auskunftsrecht $z$ A Angelegenheiten der Gesellschaft (\$\$ 118f, 131f, 172, 174 AktG).

Aktien sind Wertpapiere. Sie können formlos übertragen werden und können bei Bestehen entsprechender Märkte jederzeit verkauft und gekauft werden.

\subsubsection{Zusammenfassende Gegenüberstellung}

Die Möglichkeiten der Eigenfinanzierung sind bei einem Einzelunternehmen eng begrenzt. Das Eigenfinanzierungspotential beschränkt sich auf das Gesamtvermögen des Eigentümers bzw. auf den Anteil seines Vermögens, den er in eine einzige Anlage investieren will.

Soll im Außenverhältnis ein Einzelunternehmen erhalten bleiben, kann eine stille (Innen-) Gesellschaft gegründet werden. Für den stillen Gesellschafter ergeben sich jedoch spezielle Informationsprobleme'). Ein Rapitalgeber, der eine Anlage als stiller Gesellschafter in Erwägung zieht, sieht sich bei der Beurteilung von Chancen und Risiken seines Engagements einem Kapitalnehmer gegenüber, der einen Informationsvorsprung in bezug auf die Lage der Unternehmung und ihre Ertragsaussichten hat. Der Rapitalnehmer ist daran interessiert, dem Kapitalgeber gegenüber vertrauenswürdig zu erscheinen, aber nicht unbedingt daran, realistisch zu informieren, da sein Interesse dem Mittelzufluß gilt. Bei den beschränkten Kontrollrechten des stillen Gesellschafters können die Interessen nur zum Ausgleich kommen, wenn auf die Einlage des stillen ein relativ großer Gewinnanteil entfällt und damit das Risiko der Anlage abgegolten wird. Hat sich der Kapitalgeber als stiller Gesellschafter beteiligt, bestehen für den Kapitalnehmer An-

1) Vgl. Drukarczyk, J. (1985), S. 113 ff und Schmidt, R.H. (1981b), S. $193 f \mathrm{f}$ 
reize, die Investitionspolitik zu ändern und Risiken auf den Stillen abzuwälzen''. Auch kann er Eigenkonsum als Rosten deklarieren (fringe benefits) und insbesondere kann es für ihn interessant sein, Verluste "aufzublähen" und Gewinne auf den Zeitraum nach dem Ausscheiden des stillen Gesellschafters zu verlagern. Dies ist vor allem dann attraktiv, wenn der stille Gesellschafter bei seinem Ausscheiden nur einen nominellen Anspruch hat. Inwieweit die Ausgestaltung des Kündigungsrechts und die nur begrenzten Informationsmöglichkeiten diesen Interessen des Rapitalnehmers entgegenwirken, ist nur im Einzelfall zu beurteilen. Für Kapitalanlagen dürfte diese Finanzierungsform nur in Einzelfällen attraktiv sein, da mit dem Engagement beträchtliche Risiken verbunden sind.

Erfolgt die Kapitalanlage durch Eintritt in eine OHG, sind die Informationsrisiken für den Anleger deutlich geringer. Die Informationsrechte sind ausgebaut, zudem bestehen das Recht auf Mitwirkung in der Geschäftsührung und das Recht, die Gesellschaft $z u$ kündigen. Dadurch werden die Möglichkeiten des Kapitalnehmers begrenzt, die Informationsasymmetrie auszunutzen. Durch die erweiterten Informationsrechte insbesondere im Falle der Wahrnehmung der Geschäftsführungsbefugnis kann der Kapitalgeber die Lage der Gesellschaft relativ klar beurteilen und unter Inanspruchnahme des Ründigungsrechts Konsequenzen ziehen.

Gegenüber einem Engagement in einer stillen Gesellschaft werden die Risiken der Beteiligung für den Kapitalanleger reduziert. Allerdings ist eine solche Anlage nur für einen unternehmerisch interessierten Rapitalgeber interessant. Die Informations- und Koordinationskosten sind hoch und eine Veräußerung der Beteiligung ist insbesondere durch den Informationsvorsprung, den nun der Kapitalgeber gegenüber Dritten besitzt, kaum möglich.

Bei der RG werden die Informationsinteressen der potentiellen Anleger dadurch reduziert, daß sie nur mit ihrer Einlage haften. Sind zudem die Rommanditeinlagen relativ klein, verblei-

1) Zu solchen Anreizen aufgrund von (staatlichen) Kreditbürgschaften vgl. Chaney, P.K./Thakor, A.V. (1985), S. $169 f f$. 
ben dem Anleger Diversifikationsmöglichkeiten und das Anlagerisiko wird weiter verringert. Allerdings sind die Rommanditisten durch nur geringe Informationsrechte vor Obervorteilung durch die Romplementäre nicht geschützt.

Bestehen keine Nachschußpflichten, ist auch die Haftung der GmbH-Gesellschafter auf ihre Einlage begrenzt. Im Gegensatz zu Aktien sind die Gesellschaftsanteile an einer GmbH nur sehr beschränkt fungibel. Auch erfolgt die zerlegung des Gesellschaftskapitals in relativ große Parten, so daß nur begrenzte Diversifikationsmöglichkeiten verbleiben. Bei verhältnismäßig gut ausgebauten Informationsrechten ist jedoch in der Gesellschafterversammlung ein begrenztes unternehmerisches Engagement erforderlich. Bei der AG ist dieses nicht der Fall, da die Geschäftsführung vom Eigentum getrennt ist. Damit tritt jedoch das bereits angesprochene Agency-Problem auf. In bezug auf zusätzliche Fremdfinanzierungsmöglichkeiten hat die $A G$ gegenüber der $G \mathrm{mbH}$ den vorzug, daß die Anteile nicht kündbar sind und so von seiten der Eigentümer das Eigenkapital nicht reduziert werden kann. Da aber die Aktien leicht handelbar sind, kann der Kapitalanleger die Dauer seines Engagements dennoch flexibel gestalten, aufgrund des Kursrisikos jedoch unter Umständen mit erheblichen Kosten.

Die Gegenüberstellung der Rechtsformen zeigt, daß die Beteiligung an einer Aktiengesellschaft die für die Kapitalanleger interessanteste Anlageform darstellt. Das Informationsbedürfnis wird durch die Begrenzung der Haftung reduziert. Durch die Zerlegung des Grundkapitals und die leichte Veräußerbarkeit der Aktien können die Rapitalgeber zudem die Risiko-, Größen- und Fristenstruktur ihrer Anlagen gemäß ihren Präferenzen gestalten.

Im folgenden werden deshalb die Möglichkeiten neugegründeter sowie kleiner und mittlerer Unternehmen untersucht, die Eigenmittel zur Finanzierung von Innovationen über den Rapitalmarkt zu beschaffen. 
3. Die Eigenfinanzierung von Unternehmen über den Rapitalmarkt

Um Finanzmittel über den Kapitalmarkt beschaffen zu können, müssen die Unternehmen zwei Voraussetzungen erfüllen. Erstens müssen die Beteiligungstitel als Wertpapiere verbrieft sein. Wie die Analyse im vorigen Abschnitt ergeben hat, wird diese vorbedingung nur von Beteiligungstiteln an einer Aktiengesellschaft erfüllt, da die Anteile an Gesellschaften anderer Rechtsformen nicht als Wertpapiere verbrieft sind. Zweitens müssen die Aktiengesellschaften, die über den Rapitalmarkt Eigenmittel beschaffen wollen, an der Börse zugelassen werden.

Wie in diesem Kapitel gezeigt wurde, sind innovationsbereite und deshalb risikobehaftete Unternehmen auf die zuführung von Eigenkapital angewiesen. Die Beschaffung von Eigenmitteln über den Kapitalmarkt ist am besten geeignet, die unterschiedlichen Interessen von Kapitalgebern und Kapitalnehmern bezüglich der Risiko-, Fristen- und Größenstruktur der Anlage zum Ausgleich zu bringen. Im folgenden werden deshalb die steuerlichen Probleme, die mit der Rechtsform der Aktiengesellschaft verbunden sind, untersucht. Anschließend werden die Bedingungen, die an die Börsenzulassung gestellt werden, aus der sicht risikobehafteter kleiner und mittlerer Unternehmen diskutiert.

3.1 Die steuerlichen Folgen der Rechtsformwahl

\subsubsection{Die steuerlichen Folgen des Rechtsformwechsels}

Zur Bestimmung der steuerlichen Folgen sind drei mögliche Konstellationen eines Rechtsformwechsels zu unterscheiden'). Die erste Möglichkeit besteht in der Liquidation des bestehenden Unternehmens und anschließender Einzelübertragung der Vermögensgegenstände und schulden auf eine neu gegrūndete AG.

1) Zum Folgenden vgl. Schürmann, H./Beyer, G. (1981), S. 58ff und Haberstock, L. (1984), S. $138 f f$ 
Die steuerliche Behandlung dieser Umgründung mit Einzelrechtsnachfolge entspricht der einer Liquidation mit anschließender Neugründung. Stille Reserven sind aufzulōsen und als Veräußerungsgewinn (begünstigt) zu versteuern. Bei der obertragung fallen alle entsprechenden Verkehrsteuern (Geselischaft-, Grunderwerb- und evt. Börsenumsatzsteuer) an.

Erfolgt keine Umgründung, sondern entsteht die AG durch Umwandlung einer anderen Gesellschaft, ist für die steuerlichen Folgen auf die Rechtsnatur der untergehenden Geselischaft abzustellen. Bei der formwechselnden Umwandlung ändert sich zwar die Rechtsform, nicht aber die Rechtspersönlichkeit. Die Umwandlung einer Personengesellschaft in eine andere Personengesellschaft bzw. die Umwandlung einer Rapitalgesellschaft in eine andere Rapitalgesellschaft bleibt ohne steuerliche Konsequenzen. Bei der errichtenden Umwandlung ändert sich die Rechtspersōnlichkeit der Gesellschaft; Beispiele sind die Umwandlung einer Personengesellschaft in eine Rapitalgesellschaft und umgekehrt oder die Umwandlung eines Einzelunternehmens in eine $A G$.

Bei der Umwandlung einer Personengesellschaft oder eines Einzelunternehmens in eine Kapitalgesellschaft besteht in bezug auf die Behandlung der stillen Reserven ein wahlrecht ( $\$ 20$ II UmwStG). Das eingebrachte Betriebsvermögen kann zum Buchwert, zum Teilwert oder $z u$ einem dazwischenliegenden Wert in der Eröffnungsbilanz der Rapitalgesellschaft angesetzt werden. Liegt der Wertansatz über dem Buchwert, entstehen beim Einbringenden ermäßigt $z u$ versteuernde Veräußerungsgewinne ( $\$ \S$ 16IV, 34I EStG, Abschn. 40 I GewStR). Zudem erhöhen sich die Bemessungsgrundlage der Kapitalverkehrsteuern und der Grunderwerbsteuer, andererseits steigt auch das Abschreibungspotential der Rapitalgesellschaft. Eine Auflösung stiller Reserven ist sinnvoll, solange der Vorteil aus der steuerlichen Begünstigung des Veräußerungsgewinns und des erhöhten $\mathrm{Ab}-$ schreibungspotentials die Belastungen durch sofortige Besteuerung und höhere Verkehrsteuern übersteigt.

Im Vergleich zur ohne steuerliche Konsequenzen erfolgenden formwechselnden Umwandlung kann somit das Wahlrecht zur Auflösung stiller Reserven die anfallenden verkehrsteuerlichen Belastungen kompensieren. 


\subsubsection{Die Belastungen bei der Aktiengesellschaft}

Abgesehen von den steuerlichen Belastungen bei der Umwandlung bestehen im Vergleich zu anderen Rechtsformen auch bei der laufenden Besteuerung zusātzliche Belastungen.

Rapitalgesellschaften sind als juristische Personen selbständig vermögensteuerpflichtig (\$ II Ziff. 2a VStG). Im Vergleich zu Personengesellschaften besteht somit eine doppelte vermögensteuerliche Erfassung des Vermögens einer Rapitalgesellschaft. Zum einen wird auf der Unternehmensebene das Betriebsvermögen und zum zweiten auf Gesellschafterebene der Anteilsbesitz belastet. Zudem wird der Anteilsbesitz an Aktiengeselischaften nach dem Börsenkurs bewertet. Der so bestimmte Anteilswert geht in der Regel weit über den für nicht gehandelte Anteile (insbesondere an Personengesellschaften) festgesetzten Wert hinaus').

Außerdem sind bei der Ermittlung des körperschaftsteuerlichen Einkommens die durch den obligatorischen Aufsichtsrat verursachten Aufwendungen nur zur Hãlfte steuerlich abzugsfãhig, während Aufwendungen bei anderen Gesellschaften für freiwillig eingerichtete Beiräte voll abzugsfähig sind.

\subsection{Die Bedingungen für die Börsenzulassung}

Wertpapiere, die mit amtlicher Feststellung des Börsenpreises (amtliche Notierung) an der Börse gehandelt werden sollen, bedūrfen der Zulassung (\$ $36 I$ BörsG). Die Zulassung kann nicht vom Emittenten allein, sondern nur gemeinsam mit einem Kreditinstitut, das an einer inländischen Börse zum Handel zugelassen ist, beantragt werden (\$ $36 I I$ BörsG).

Bis zur Neufassung des Börsen-Gesetzes zum 1.1 .1987 war es Sache der Börsen, die Zulassungsbestimmungen so festzulegen, daß dem Publikum ein zutreffendes Urteil über Emittent und Wertpapier ermöglicht und eine obervorteilung des Publikums verhindert wird (\$ 36 III BörsG). Da das emissionsbegleitende Rreditinstitut Börsenmitglied sein muß und als solches die Zulassungsentscheidung auch mitzuverantworten hat, ist das

1) Vgl. Ertl, B. (1983), S. 137 und Schürmann, W./Beyer, G. (1981), S. 84 
Finden eines Emissionshauses das entscheidende Problem bei der Börseneinfūhrung, zumal dieses Rreditinstitut auch fūr die Richtigkeit des zur Information des Publikums veroffentlichten Börsenprospekts haftet ( $\$ \$ 36 I I, 45$ BörsG). Neben den formalen, sehr kostenintensiven Anforderungen an Prospektund Publizitätspflicht bestehen deshalb bedeutende faktische Marktzutrittshemmnisse, die durch die Furcht der Banken vor Fehlschlägen verschärft werden. Als "kapitalmarktreif"i) werden nur solche Unternehmen betrachtet, die ein Eigenkapital von 5-10 Millionen DM und einen Umsatz von 50 Millionen DM haben. Aufgrund dieser Anforderungen wird kleineren kapitalsuchenden Unternehmen der Zutritt zum Kapitalmarkt praktisch verwehrt. Nur große, etablierte Aktiengesellschaften gelten nach diesen Bedingungen als "kapitalmarktreif" und sind auch in der Lage, die Kosten der Markteinführung zu tragen ${ }^{2}$ ). Die neben dem amtlichen Markt bestehenden Freiverkehrssegmente sind zwar formal weniger strikt reglementiert, doch sind auch hier begleitende Emissionsbanken zur Einbeziehung in diese Marktabschnitte erforderlich. Da diese jedoch an Randidaten für diese Marktsegmente die im wesentlichen gleichen Qualitätsanforderungen stellen, besteht faktisch keine Abstufung der Marktabschnitte. Dies zeigt sich auch daran, daß viele Aktien an den verschiedenen Börsen in unterschiedlichen Marktsegmenten gehandelt werden ${ }^{3}$ ).

\section{$\underline{4}$.} FINANZIERUNG DURCH NICHT-GESELLSCHAFTSRECHTLICHE BETEILIGUNGSTITEL

Die Ausgabe von Genußscheinen bietet auch nicht emissionsfähigen Unternehmen die Möglichkeit der Beschaffung von Finanzierungsmitteln über organisierte Kapitalmärkted). Je nach Ausgestaltung des Genußscheins, für den es keine Legaldefinition gibt, können die mit diesem Finanzierungsinstrument erworbenen Mittel den Charakter von Eigen- oder Fremdkapital besitzen.

1) Delbrück, J. (1983), S. 141ff

2) Vgl. Hunsdiek, D./Kokalj, L. (1985), S. 842ff

3) Vgl. Albach, H./Hunsdiek, D./Kokalj, L. (1986), S. $66 \mathrm{ff}$

4) Vgl. Vollmer, L. (1983), S. $445 \mathrm{ff}$ und derselbe (1984), S. 331ff 
Als Parameter für die Gestaltung des Genußrechtsverhältnisses stehen dem kapitalsuchenden Unternehmen die Regelung des Gewinnanspruchs, der Verlustteilnahme und der Beteiligung am Liquidationserlös, die Gewährung von Bezugs- und Mitwirkungsrechten sowie die Ausgestaltung der Beendigung des Genußrechtsverhältnisses zur Verfügung .

Steuerlich sind für Rapitalgesellschaften solche "Genußrechte, mit denen das Recht auf Beteiligung am Gewinn und Liquidationserlös" (\$ 8III RStG) verbunden ist, als Eigenkapital zu qualifizieren. Auf sie erfolgende Ausschüttungen mindern den Ertrag nicht. Für von Personengesellschaften und Einzelunternehmen ausgegebene Genußscheine ist über eine Analogie zur steuerlichen Behandlung der stillen Gesellschaft eine entsprechende Behandlung anzunehmen').

Genußscheine können zwar unabhängig von der Rechtsform des emittierenden Unternehmens als Instrument der Rapitalbeschaffung eingesetzt werden, ihre zulassung zum Börsenhandel hat aber den gleichen Bedingungen zu entsprechen wie die zulassung von Aktien'). Für kleine und mittlere Unternehmen bestehen deshalb bei der Genußscheinfinanzierung ähnliche Marktzugangsprobleme wie bei der Kapitalbeschaffung durch die Ausgabe von Beteiligungsrechten.

\section{ZUSAMMENFASSUNG}

In diesem Kapitel wurden die Möglichkeiten der Unternehmensfinanzierung unter zwei Aspekten untersucht. Zum einen wurde der Einfluß der Besteuerung auf die Rapitalbildung und die Auswahl der Finanzierungsformen analysiert. Daran anschlieBend wurden die Möglichkeiten der Rapitalbeschaffung innovationsbereiter Unternehmen diskutiert.

Die Untersuchung der steuerlichen Institutionen zeigt, daß die staatliche Förderung der Kapitalbildung für die Akkumulation von Gründungskapital nur geringe Bedeutung hat. Dagegen

1) Vgl. Hoffmann, W.-D. (1985), S. $392 f$

2) Vgl. § 35 BörsG und $\$ 54$ Börso der Baden-Württembergischen Wertpapierbörse zu stuttgart 
wird das aus Gewinnen und sonstigem Einkommen stammende Finanzierungspotential durch die Belastung mit steuern und anderen Zwangsabgaben drastisch reduziert.

Der Vergleich der steuerlichen Behandlung alternativer Finanzierungswege ergibt eine eindeutige Präferenz für die Fremdfinanzierung. Dennoch verursacht auch die Fremdfinanzierung Verzerrungen im Investitionskalkül, da nicht die gesamten Opportunitätskosten des Rapitaleinsatzes steuerlich absetzbar sind. Es wurde deshalb im weiteren untersucht, ob innovative, risikobehaftete Aktivitäten stärker als andere Investitionen durch die Möglichkeiten der Kapitalbeschaffung gehemmt werden.

Die Risikoeinstellung der Banken bei der Rreditvergabe kann zwar nicht direkt beobachtet werden. Ober die Rreditausfallquoten können jedoch Rückschlüsse auf das Entscheidungsverhalten gezogen werden. Die modelltheoretische Analyse ergibt, daß bei extremer Risikoaversion der Kapitalgeber die Rreditgewährung durch das Volumen der vom Rreditnehmer gestellten sicherheiten bestimmt wird. Von einer solchen Beschränkung der Kreditgewährung sind vor allem innovationswillige neugegrūndete sowie kleine und mittlere Unternehmen betroffen, da erstens das Projekt, das finanziert werden soll, relativ risikoreich ist und sich zum zweiten die erbrachten Vorleistungen nicht in dinglichen sicherheiten sondern in know-how niederschlagen. Die empirischen Daten weisen auf eine ausgeprägte Risikoscheu der Banken hin. Die Rreditausfälle liegen deutlich unter einem Prozent bezogen auf das Volumen der gewährten Kredite. Die Möglichkeiten der Fremdfinanzierung von Innovationen sind somit geringer als die Möglichkeiten der Beschaffung von Fremdkapital für andere Investitionsobjekte. Innovationsbereite Unternehmen sind deshalb verstärkt auf die Zuführung von Eigenkapital angewiesen.

Die Diskussion der Wege, über die Beteiligungskapital beschafft werden kann, ergibt, daß die Eigenfinanzierung über den Kapitalmarkt in der Rechtsform der Aktiengesellschaft die kostengünstigste Form der Beschaffung von Finanzierungsmitteln darstellt. Bei dieser Ausgestaltung der Eigenfinanzierung sind die Kosten der Transformation von Fristen, Risiken und Losgrößen am niedrigsten. Die Analyse der Unternehmensbesteuerung ergibt jedoch, daß die steuerliche Behandlung al- 
ternativer Rechtsformen nicht neutral ist und da $\beta$ insbesondere die Umwandlung in eine Aktiengesellschaft steuerliche Belastungen verursachen kann. Die öffentliche Emission von Aktien und deren Handel bedarf der zulassung. Die gesetzlich fixierten bzw. die in den Börsenordnungen festgelegten Kriterien stellen jedoch für innovationsbereite Unternehmen erhebliche Marktzugangsschranken auf. Da Anlegerschutz vor allem durch Risikobeschränkung erreicht werden soll, ist der Marktzutritt für innovationsbereite neugegründete sowie kleine und mittlere Unternehmen kaum möglich, da ihre Entwicklung stark risikobehaftet und nur schwer einzuschätzen ist.

Ein zusätzliches Marktzugangshemmnis stellen die gesetzlichen Mitbeștimmungsregelungen dar. Außer für Aktiengesellschaften ist der Umfang der Mitbestimmungsrechte in allen Unternehmensrechtsformen größenabhängig. Bei Aktiengesellschaften ist jedoch bereits die Rechtsform konstituierendes Merkmal für die Vertretung von Arbeitnehmern im Aufsichtsrat ( $\$ 76$ I BetrVerfG). Mit der Umwandlung in eine Aktiengesellschaft müssen auch kleine und mittlere Unternehmen einen mitbestimmten Aufsichtsrat bilden'1).

Die für die Eigenkapitalbeschaffung innovativer Aktiengesellschaften genannten strukturellen Probleme gelten auch für die Finanzierung durch nicht-gesellschaftsrechtliche Beteiligungstitel. Grund hierfür ist ebenfalls die mangelhafte hierarchische Abstufung der einzelnen Marktsegmente.

1) Vgl. Werner, W./Kindermann, E. (1981), S. $23 f$ 
TEIL 3: MARKTWIRTSCHAFTLICHE INNOVATIONSPOLITIR: MOGLICHKEITEN - GRENZEN - RONZEPTE

G. MOGLICHREITEN UND GRENZEN DER INNOVATIONSPOLITIR

\section{I.} GEGENSTAND DER INNOVATIONSPOLITIR

Zu Beginn dieser Arbeit wurde jede wirtschaftliche Verwertung neuartiger Rombinationen von Angebot und Nachfrage, seien es neue Produkte oder Produktqualitäten, Produktionsverfahren oder organisationsformen, als Innovation definiert. Innovationspolitik bezieht sich deshalb nicht nur auf die Durchsetzung technischer Neuerungen sondern auf jede neuartige wirtschaftliche Rombination. Innovationspolitik umfaßt alle staatlichen Maßnahmen auf mikroökonomischer Ebene' ', die Ausmaß und Richtung der Innovationsaktivitäten der privaten Wirtschaftssubjekte beeinflussen ${ }^{2}$ ). Diese Begriffsfassung schließt somit sowohl Maßnahmen ein, die das Entscheidungsverhalten bezüglich eines bestimmten Projekts beeinflussen sollen, als auch strukturpolitisch motivierte und allgemein angebotsorientierte Eingriffe.

Träger der Innovationspolitik sind alle staatlichen Ebenen, die Maßnahmen treffen, welche die Innovationsbedingungen bestimmen. In der Bundesrepublik Deutschland sind dies Bund, Länder und Gemeinden einschließlich ihrer zu diesem Zweck geschaffenen privatrechtlich organisierten Unternehmen bzw. der mit den entsprechenden Aufgaben betrauten Finanzintermediäre. Mittel der Innovationspolitik sind alle staatlichen Interventionen, welche die Beeinflussung der einzelwirtschaftlichen Innovationsentscheidungen zum ziel haben. Beispiele sind zum einen die fiskalischen Instrumente wie steuergesetze, $\mathrm{Zu}-$ schüsse, Bürgschaften etc. sowie die regulierenden Eingriffe auf Güter-, Rapital- und Arbeitsmärkten.

1) Somit sind konjunkturpolitische Aktivitäten, die Innovationsentscheidungen auch beeinflussen, in dieser Definition nicht enthalten.

2) Vgl. Reich, R.B. (1983), S. 3 
Das ziel dieser Politik ist die Erhöhung und Beschleunigung der Innovationstätigkeit, d.h. der wirtschaftlichen Verwertung neuer Produkte und Produktqualitäten, neuartiger Produktionsverfahren und organisationsformen.

Synonym zum Begriff der Innovationspolitik werden in der wirtschaftspolitischen Diskussion die Termini "Neue Industriepolitik" bzw. "Neue Industrie- und Forschungspolitik" oder auch "Forschungs- und Technologiepolitik" verwandt). Das hinter diesen Begriffen stehende Innovationsverständnis weicht jedoch von der hier vertretenen Definition der Innovation durch seine Ronzentration auf technische Neuerungen ab. Daß dies nicht ohne Auswirkungen auf die ziele und Mittel der Innovationspolitik ist, wird bei der Diskussion von Konzepten und Programmen zur Innovationspolitik deutlich.

\section{VORAUSSETZUNGEN FOR EINE INNOVATIONSPOLITIR}

\section{Notwendige Bedingung}

In einem marktwirtschaftlichen system, in dem Richtung und Ausmaß der wirtschaftichen Aktivitäten vom Verhalten der durch Marktsignale gesteuerten privaten Wirtschaftssubjekte bestimmt werden, ist das Vorliegen von Marktversagen eine notwendige Bedingung für das Ergreifen staatlicher Maßnahmen.

Bevor auf einzelne, insbesondere in der politischen Diskussion vorgebrachte Begründungen für eine Innovationspolitik eingegangen wird, sollen Bedingungen und Voraussetzungen für eine optimal korrigierende Politik und für die Möglichkeiten ihrer praktischen Realisierung analysiert werden.

\subsection{Optimalitätsbedingungen}

Als Beispiel zur Ableitung der Bedingungen für eine optimal korrigierende Politik wird der Fall externer Effekte gewählt. Es wird angenommen, daß sich die Unternehmen nicht alle Er-

1) Vgl. Issing, 0. (1986), S. 2 
träge ihrer Innovationsaktivitäten aneignen können. Somit ist die durch die Marktteilnehmer offenbarte maximale marginale Zahlungsbereitschaft geringer als dies unter Berücksichtigung der gesamten volkswirtschaftlichen Erträge der Innovationstätigkeit der Fall wäre.

Abbildung G.1: Wohlfahrtseffekte von Allokationsstörungen

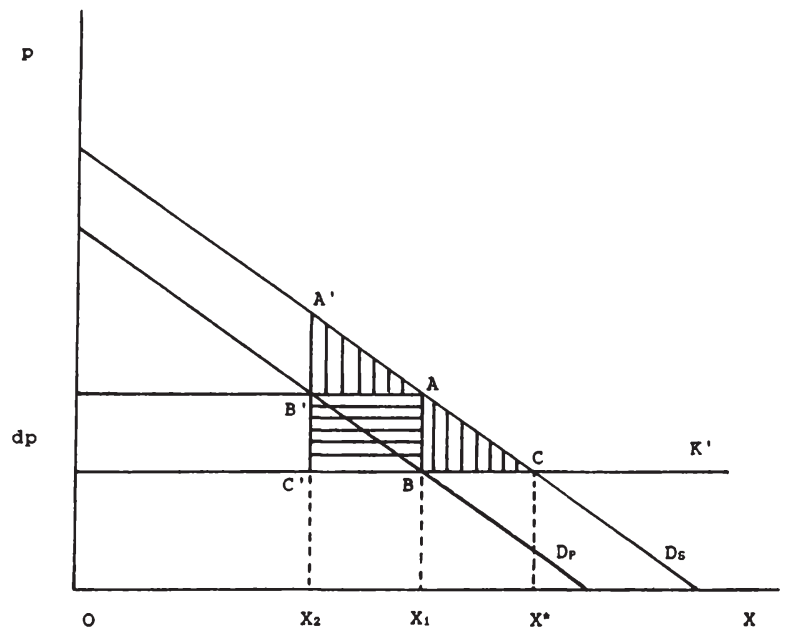

In Abb. G.1 sind die Kurven der maximalen marginalen Zahlungsbereitschaft für die Menge $X$ an Innovationstätigkeit ohne $\left(D_{p}\right)$ und mit $\left(D_{s}\right)$ Berücksichtigung der externen Erträge eingezeichnet. Um die Darstellung zu erleichtern, werden ein linearer verlauf dieser Nachfragekurven und konstante Grenzkosten $K^{\prime}$ unterstellt. Die Gültigkeit der folgenden Ableitungen wird durch diese Vereinfachungen nicht eingeschränkt. Der durch die allokative störung verursachte Wohlfahrtsverlust $\Delta \mathrm{W}$, der aufgrund der konstanten Grenzkosten nur ein Verlust an Ronsumentenrente ist, beläuft sich auf die durch das Harberger-Dreieck $A B C$ abgebildete Fläche' ${ }^{1}$ :

$$
\Delta \mathrm{W} \hat{=} \mathrm{ABC} \text {. }
$$

1) Vgl. Harberger, A.C. (1971), S. $7 f$ 
Dieses Dreieck läßt sich formal in folgender Weise darstellen:

(G.1) $\quad \Delta W=\not / 2\left(X^{\star}-X_{1}\right) \overline{A B}$

$$
=x_{2}\left(x^{\star}-x_{1}\right) d p \text {. }
$$

Wird die Mengenstörung dx definiert als

(G.2)

$$
a \equiv X^{\star}-X_{1},
$$

dann gilt:

(G.3)

$$
\begin{aligned}
\Delta \mathrm{w} & =/ 2 \mathrm{a} \cdot \mathrm{dp} \\
& =/ 2 \mathrm{a}^{2} \cdot \mathrm{dp} / \mathrm{dX}
\end{aligned}
$$

bzw. bezogen auf die Preisstörung dp, mit

(G.4)

$$
d \equiv p_{1}-p^{\star}
$$

(G.5)

$$
\Delta \mathrm{W}=1 / 2 \mathrm{~d}^{2} \cdot \mathrm{dX} / \mathrm{dp} \text {. }
$$

Um die Allokationsstörung zu beheben, kann den Nachfragern eine subvention s gewährt werden, mit der die mangelnde Appropriierbarkeit in Höhe von dp ausgeglichen werden soll.

Der Wohlfahrtseffekt nach störung und Rorrekturmaßnahme beträgt dann:11

$$
\begin{aligned}
& \Delta \mathrm{W}=1 / 2 \mathrm{~d}^{2} \cdot \mathrm{dX} / \mathrm{dp}-1 / 2 \mathrm{~s}\left(\mathrm{X}^{\star}-\mathrm{X}_{1}\right) \\
& =1 / 2 d x(d-s) \quad 2) \text {. }
\end{aligned}
$$

Daraus folgt:

$$
\Delta \mathrm{W}=0,
$$

1) Vgl. Brennan, G./McGuire, T. (1975), S. $206 f$ und Folkers, C. (1987), S. $175 f$

2) Der Wohlfahrtsgewinn bei einer Subventionierung mit dem Betrag s pro Einheit von $X$ beträgt $/ 2 s$ pro Einheit von $X$ für den Bereich der störung $X^{\star}-X_{1} ;$ vgl. Abb. G.1. 
d.h. die störung wird voll kompensiert, wenn gilt:

(G.7) $\quad d=s$.

D.h. werden die externen Effekte durch eine Subvention zugunsten der Innovatoren internalisiert, so wird die Allokationsstörung beseitigt.

\subsection{Die Informationserfordernisse}

Im vorangehenden Abschnitt wurde für eine störung auf einem bestimmten Markt die Optimalbedingung zur Korrektur der Allokationsineffizienz abgeleitet. Um die geeignete Maßnahme zur Behebung der störung treffen zu können, müssen den Entscheidungsträgern Richtung und Ausmaß der Abweichung des Marktergebnisses vom optimum bekannt sein.

Die Konsequenzen eines unzureichenden Informationsstandes für die Zielkonformität einer Maßnahme lassen sich aus Abb. G.1 ablesen. Kann der Entscheidungsträger zwar eine Marktstörung beobachten, ist ihm aber die Richtung der Abweichung vom optimum unbekannt, so ist der mögliche Gewinn einer bestimmten Maßnahme geringer als der verlust, der eintritt, wenn die Maßnahme in die falsche Richtung zielt. Wäre bei der in Abb. G.1 dargestellten Ronstellation statt einer subventionierung eine Besteuerung in gleicher Höhe erfolgt, hätte sich anstelle eines Wohlfahrtsgewinns in Höhe des Dreiecks ABC eine Wohlfahrtsverschlechterung in Höhe der Fläche A'ABC' ergeben. Sind eine positive oder negative Abweichung vom optimum gleich wahrscheinlich, so ist der Erwartungswert des Wohlfahrtseffekts einer Maßnahme bei risikoneutralem Verhalten des Entscheidungsträgers negativ. Der Grund für dieses Ergebnis kann für die Ronstellation in Abb. G.1 aus Gleichung (G.3) bzw. (G.5) entnommen werden. Der Wohlfahrtsverlust steigt überproportional mit dem Ausmaß der störung.

Wird der Wohlfahrtsverlust einer Marktstörung allgemein als die Fläche zwischen der einkommenskompensierten Nachfragekurve und der Grenzkostenkurve formuliert'):

1) Vgl. Brennan, G./Buchanan, J.M. (1983), S. 104 und Folkers, C. (1987), S. $172 f$ 
(G.8) $\quad \Delta W=\left|\int_{x^{*}}^{x}\left[D(X)-K^{\prime}(x)\right] d x\right|$,

so gilt bei einer Mengenstörung

$$
d x=\left|x^{\star}-x\right|
$$

$$
d(\Delta w) / d X=\left|\left[D(X)-K^{\prime}(X)\right]-\left[D\left(X^{\star}\right)-K^{\prime}\left(X^{\star}\right)\right]\right| .
$$

Bei der optimalen Menge $x^{\star}$ gilt:

$$
D\left(X^{\star}\right)=K^{\prime}\left(X^{\star}\right)
$$

d.h. Preis = Grenzkosten, und damit:

$$
d(\Delta w) / d x=\left|\left[D(X)-K^{\prime}(X)\right]\right|
$$

Somit gilt:

$$
d(\Delta w) / d x>0
$$

für

(G.14a) $d x=x^{\star}-x>0$ und $D(X)-K^{\prime}(X)<0$ bzw.

(G.14b) $d x=x^{*}-x<0$ und $D(x)-R^{\prime}(x)>0$

und

(G.15) $d^{2}(\Delta W) / d^{2}>0$

für

(G.16)

$$
d X=X^{\star}-X>(<) \quad 0 \text { und } D^{\prime}(X)-R^{\prime \prime}(X)>(<) 0 \text {. }
$$

Der Wohlfahrtsverlust $\Delta \mathrm{W}$ wächst somit immer überproportional mit der störung $d x$, wenn die steigung der Nachfragekurve geringer ist als die steigung der Grenzkostenkurve. Es sind zwar Fäle denkbar, in denen diese Bedingung verletzt sein könnte, doch sind diese von keiner praktischen Bedeutung' '. Die Folgerungen für die politischen Aktivitäten in einer solchen situation der Informationsarmut sind eindeutig. Bei negativem Erwartungswert einer Maßnahme ist diese zu unterlassen. Das bedeutet aber nichts anderes, als daß eine erstbeste

1) Denkbar wăren zum Beispiel bereichsweise steigende Nachfragekurven (snobeffekt). 
Welt unterstellt wird, d.h. es werden die Bedingungen des Pareto-Optimums als erfüllt vorausgesetzt. Diese Unterstellung ist zwingend, wenn das erwartete Ausmaß der störung bei Informationsarmut betrachtet wird. Da keine Informationen über Richtung und Ausmaß der störung vorliegen, ist jede Abweichung vom optimum gleich wahrscheinlich. Die störungen streuen symmetrisch um das optimum. Für den Erwartungswert einer störung $\mathrm{dX}=\mathrm{X}^{\star}-\mathrm{X}$ gilt somit:

\section{(G.17) $\quad E(d X)=0$.}

Stellt der Erwartungswert das Entscheidungskriterium dar, d.h. handelt der Entscheidungsträger risikoneutral, dann sind die Optimalitätsregeln einer erstbesten Politik zu unterstellen und eine Politik des Zweitbesten ist bei diesem Informationsstand zu unterlassen'1).

Ist jedoch $\mathrm{E}(\mathrm{dX}) \neq 0$ oder liegt keine Risikoneutralität vor, sind die eben gezogenen schlußfolgerungen zu modifizieren.

Ist der Erwartungswert von null verschieden, bedeutet dies, daß konkrete Informationen über Richtung und Ausmaß der Allokationsstörung vorliegen').

Gewichtet der Entscheidungsträger in seiner Nutzenfunktion die möglichen positiven Wirkungen einer Maßnahme höher als die negativen, so ist auch bei einem Erwartungswert $E(d X)=0$ der Erwartungsnutzen einer Maßnahme größer null.

$\mathrm{Ob}$ unter diesen Bedingungen von den aus Gleichung (G.17) abgeleiteten schlußfolgerungen abgewichen werden soll, hängt von der Einschätzung der Qualität der Informationen durch den Entscheidungsträger, von seinen Erfahrungen mit Markteingriffen sowie von seiner Risikoneigung im Vergleich zu den Kosten eines solchen Eingriffes $a b^{3}$ ).

Die Konsequenzen aus den Erörterungen der letzten Abschnitte formuliert $\mathrm{Ng}$ in einer nach Informationsstand und Risikonei-

1) Vgl. Brennan, G./McGuire, T. (1975), S. $207 f f$ und Ng, Y. -K. (1976), S. $4 f$

2) Vgl. Folkers, C. (1987), S. $177 f$

3) Vgl. Brennan, G./McGuire, T. (1975), S. $208 f$ 
gung differenzierenden Politik des Drittbesten ${ }^{1}$. Erst- und zweitbeste Lösungen bilden dabei die Grenzfälle der strategien innerhalb einer Politik des Drittbesten ${ }^{2}$ ).

Die Annahme einer erstbesten, d.h. verzerrungsfreien Welt ist aufgrund der zahlreichen zu beobachtenden Marktstörungen unrealistisch. Besteht jedoch Informationsarmut, d.h. sind Ausmaß und Richtung der Marktstörung unbekannt, dann besteht, wie die obige Analyse ergibt, die optimale Politik bei risikoneutralem und risikoaversem Entscheidungsverhalten darin, erstbeste Lösungen als gegeben zu unterstellen und auf korrigierende Maßnahmen zu verzichten.

Liegen auf dem Markt korrigierbare Verzerrungen vor und sind hinreichende Informationen über deren Ausmaß und Richtung vorhanden, dann sind optimal korrigierende Maßnahmen zur Erreichung einer erstbesten Lösung (z.B. Erhebung einer Pigousteuer) zu treffen, wenn die Rosten des Eingriffs von seinen erwarteten Nutzen überkompensiert werden ${ }^{3}$ ).

Rann dagegen die Wohlfahrt nicht dadurch maximiert werden, daß, wo möglich, erstbeste Lösungen realisiert werden, d.h. es liegt eine stōrung im Sinne der Theorie des Zweitbesten vor'), dann kann nur über eine gezielte Abweichung von den erstbesten Regeln auf den mit dem betrachteten Markt durch Substitutions- und/oder Romplementaritätsbeziehungen verbundenen Märkten ein Wohlfahrtsmaximum erreicht werden. Eine solche Politik wirft jedoch außerordentliche Informationsbedürfnisse auf, da sie die Renntnis aller Interdependenzen zwischen den Märkten voraussetzt'). Im Rahmen einer Theorie des Drittbesten ist deshalb nur bei hinreichend spezifizierten Informationen über die Märkte, die mit dem betrachteten Markt verbunden sind, eine Abweichung von der erstbesten Regel zulässig, ansonsten ist auf das Ergreifen von Maßnahmen im Sinne der Theorie des $\mathrm{zweitbesten} z$ verzichten und eine erstbeste Lösung anzustreben ${ }^{6}$ ).

1) Vgl. Ng, Y. $-\mathrm{K} .(1976)$, S. $3 \mathrm{ff}$

2) $\mathrm{Vgl} . \mathrm{Ng}, \mathrm{Y} .-\mathrm{K} .(1976), \mathrm{S} .6$

3) Vgl. Turvey, R. (1963), S. $312 f$

4) Vgl. Lipsey, R.G./Lancaster, K. (1956/57), S. $11 \mathrm{ff}$

5) Vgl. Sohmen, E. (1976), S. $425 f f$

6) Vgl. Ng, Y.-K. (1977), S. 6 ff und Folkers, C. (1987), S. 178 
In der Theorie des Drittbesten bleibt somit aufgrund der Einbeziehung von Informationserfordernissen sowie der Beachtung von Rosten staatlicher Eingriffe wenig Raum für umfassende Konzepte staatlicher Rorrekturmaßnahmen ${ }^{1}$ ). Informationserfordernisse und Administrationskosten reduzieren aus der sicht der Theorie des Drittbesten die Möglichkeiten der Wirtschaftspolitik auf eine "unambitious corrective policy"2).

Die Problematik der Interdependenzen substitutiver und/oder komplementärer Natur mit anderen Märkten soll anhand von $\mathrm{Abb}$. G.2 kurz skizziert werden ${ }^{3}$ ).

Abbildung G.2: Second-best
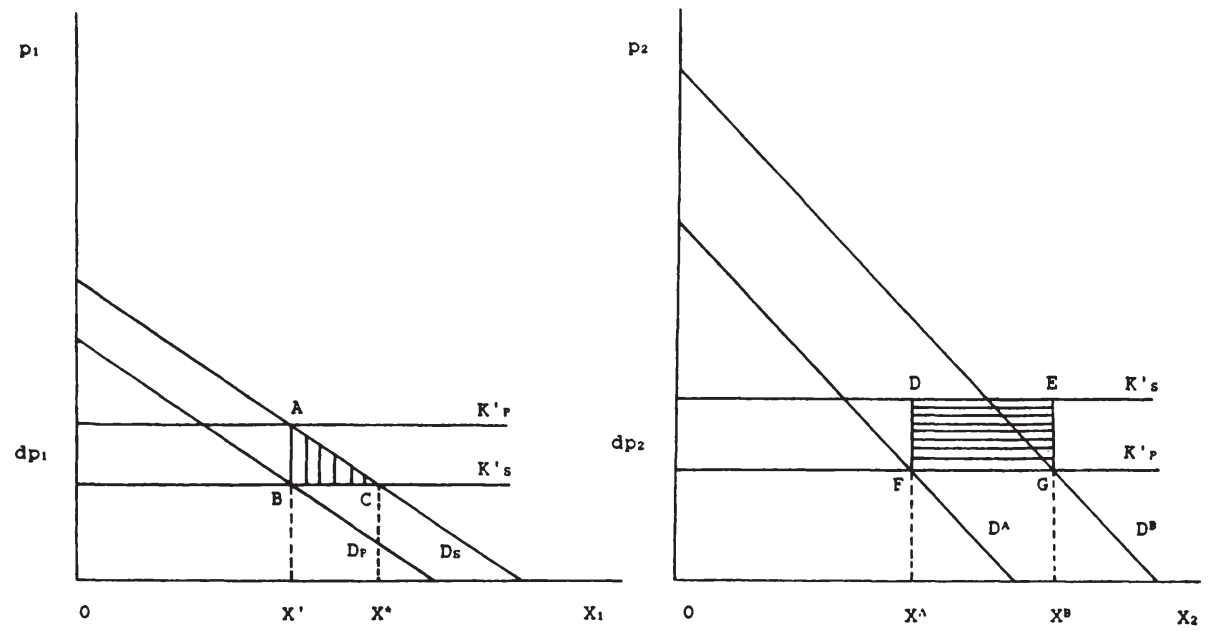

Die Märkte für Gut $x_{1}$ bzw. $x_{2}$ sind unter der ceteribus-paribus-Annahme dargestellt; die Preise aller anderen Güter sowie das Einkommen seien konstant.

1) Vgl. Folkers, C. (1987), S. 178

2) Brennan, G./McGuire, T. (1975), S. 209; vgl. dazu auch die Ergebnisse in Capitelli, R./Müller, H.B. (1986), S. $536 \mathrm{ff}$ für die Wirkung der Berücksichtigung von Administrationskosten staatlicher Interventionen zur Internalisierung externer Effekte in einem Modell des Innovationswettbewerbs.

3) Vgl. Boadway, R.W./Wildasin, D.E. (1984), S. $176 \mathrm{ff}$ 
Auf dem Markt für Gut $x_{1}$ liegen wie bei der isolierten Einzelmarktbetrachtung (positive) externe Effekte vor. Durch die Subventionierung von Gut $x_{1}$ mit einem Betrag von $d p=\overline{A B}$ kann diese Allokationsstōrung behoben werden. Dadurch entsteht auf dem Markt $f$ ür $\mathrm{X}_{1}$ ein Wohlfahrtsgewinn in Höhe des HarbergerDreiecks ABC.

Gut $X_{2}$ sei nun komplementār zu $X_{1}$. Die subventionierung von $\mathrm{X}_{1}$ und die resultierende Nachfrageerhöhung bewirken eine Verschiebung der Nachfrage nach Gut $X_{2}$ nach außen $\left(D^{1} \Rightarrow D^{2}\right)$ und somit auch für $X_{2}$ eine Ausdehnung der nachgefragten Menge. In der Rostenfunktion des Anbieters von $X_{2}$ seien aber nicht alle Kostenbestandteile enthalten, d.h. es bestehen bei der Produktion von Gut $\mathrm{X}_{2}$ negative Externalitäten. Durch die Rorrekturmaßnahme auf dem Markt für $\mathrm{x}_{1}$ und die dadurch ausgelöste Mengenanpassung bei Gut $X_{2}$ entsteht auf dem Markt für $\mathrm{X}_{2}$ ein zusätzlicher Wohlfahrtsverlust von DEFG. Da in diesem Fall DEFG größer als der Wohlfahrtsgewinn auf dem Markt für $\mathrm{x}_{1}$ ist, führt die Rorrekturmaßnahme durch die Nicht-Beachtung der Komplementaritätsbeziehungen $z$ wischen $X_{1}$ und $X_{2}$ zu einer Wohlfahrtsverschlechterung. Wären die Beziehungen zwischen den beiden Gütern dagegen substitutiver Natur gewesen, so wäre die Folge der subventionierung von $x_{1}$ ein Nauhfragerückgang bei $X_{2}$ und somit ein zusätzlicher Wohlfahrtsgewinn gewesen.

Dieses Beispiel zeigt, daß nicht nur die Kenntnis von Richtung und Ausmaß der störung auf dem betrachteten Markt sondern auch Informationen über Art und stärke von Interdependenzen mit anderen Märkten eine notwendige Voraussetzung für eine wirksame Politik sind.

\subsection{Spezielle Probleme der Innovationspolitik}

Wie eingangs dieses Kapitels definiert, verfolgt die Innovationspolitik das ziel, die Aktivitäten zur wirtschaftlichen Verwertung neuer Produkte und Produktqualitäten, neuartiger Produktionsverfahren und organisationsformen zu stärken. Sie ist somit auf neue, d.h. bis dato unbekannte Rombinationen von Produktionsverfahren und Bedürfnissen gerichtet. Wären diese Rombinationen bekannt, wären sie nicht neu und somit 
keine Innovationen. Um eine induzierende, d.h. eine veranlassend gedachte Politik ${ }^{1}$, formulieren zu können, muß aber der Fördergegenstand bekannt sein. Eine so verstandene Innovationspolitik setzt bei den planenden Instanzen die Renntnis des Neuartigen, Unbekannten voraus. Dies ist aber ein unauflösbarer logischer Widerspruch. Eine solche Politik ist von der Natur der Sache her unmöglich"). "Das ... Unbekannte ist schlechterdings nicht förderbar."3)

Eine induzierend angelegte Innovationspolitik erfordert ein Lenkungswissen, das aufgrund seiner Komplexität und der Eigenschaften von Innovationen überhaupt nicht erworben werden kann.

Das benötigte Lenkungswissen geht in zweierlei Hinsicht über die am Markt beobachtbaren Daten hinaus. Zum einen wird nur ein Teil des für ökonomische Entscheidungen relevanten und in ihnen verarbeiteten Wissens artikuliert, wird zum Datum und beobachtbar. Ober Preise, Mengen und Kostenverläufe hinaus gehen zahlreiche weitere Informationen in ökonomische Entscheidungen ein. Ausschlaggebend für eine bestimmte Transaktion ist für ein Wirtschaftssubjekt, wie es diese im Rontext seiner eigenen Plāne wahrnimmt'). Die am Markt beobachtbaren Daten bilden nur einen Teil dieses Zusammenhangs. Das übrige Wissen des Entscheidungsträgers, sein "general expectational scenario"J) wird nicht explizit, bleibt unausgesprochen').

Zum zweiten erfordert eine gestaltende Innovationspolitik Lenkungswissen über Bereiche, Richtung und Ausmaß von Innovationsmöglichkeiten. Dieses Wissen ist aber in einer Wirtschaft a priori nicht vorhanden und kann von den politischen Entscheidungsträgern somit auch nicht erworben werden'). Es wird erst durch vorstoßende Wettbewerbsaktionen und darauf

1) Vgl. Streit, M.E. (1984), S. 35

2) Vgl. Dürr, E. (1984), S. 407

3) Zeppernick, R. (1985), S. 74

4) Vgl. Lavoie; D. (1985), S. 114

5) Lavoie, D. (1985), S. 113

6) $\mathrm{Zu}$ dieser Unterscheidung von "articulate" und "inarticulate" bzw. "tacit knowledge" vgl. Lavoie, D. (1985), S. $51 \mathrm{ff}$ und die dort angegebene Literatur.

7) Vgl. Staudt, E. (1985), S. $464 f$ 
folgende Anpassungsreaktionen erzeugt. Der Wettbewerb ist somit ein Entdeckungsverfahren, wobei die Veränderungen in den relativen Preisen den Marktteilnehmern als Indikatoren für die Richtung von Verhaltensänderungen gelten ${ }^{1}$ '.

\section{Hinreichende Bedingung}

Die Untersuchung der notwendigen Bedingung für staatliche Aktivitäten ergab bereits eine relativ starke Eingrenzung der Möglichkeiten einer staatlichen Innovationspolitik. Grund dafür sind insbesondere die für eine zielgerechte Formulierung der Interventionen erforderlichen Informationsbedürfnisse. Ist ein Marktversagen festgestellt und haben die geplanten Maßnahmen bei dem gegebenen Informationsstand einen positiven Erwartungswert, ist also die notwendige Bedingung erfüllt, so reicht dies jedoch noch nicht aus, um den staatlichen Eingriff durchzuführen. Zusätzlich ist zu prüfen, ob die Intervention dauerhaft $z u$ einer Wohlfahrtssteigerung führt. Es ist also zu untersuchen, ob die angestrebte Allokationsverbesserung nicht durch marktliche und politisch-ökonomische Reaktionen, die auf die Maßnahme folgen, verhindert oder gar in ihr Gegenteil verkehrt wird. Auf einer zweiten Ebene ist somit die Frage zu stellen, ob dem Marktversagen nicht ein staatsversagen folgt und dann trotz des Marktversagens das unkorrigierte Marktergebnis besser als das korrigierte ist.

\section{$2.1 \quad$ Kapitalisierungseffekte}

Jede staatliche Maßnahme, die die Förderung einer bestimmten wirtschaftichen Aktivität zum ziel hat, wirkt diskriminierend in dem sinne, "daß ex ante gleichgestellte Wirtschaftssubjekte durch den Eingriff in ökonomisch ungleiche Positionen gelangen."2) Diese Diskriminierung hat marktliche und po-

1) Vgl. Hayek, F.A. von (1968), insbes. S. $7 \mathrm{ff}$ und derselbe (1975), S. $13 \mathrm{ff}$ sowie Stackelberg, H. von (1949), S. $197 \mathrm{ff}$

2) Mann, H. (1987), S. 176; vgl. auch Buchanan, J.M. (1980), S. 11: "political interference with markets creates differentially advantageous positions". 
litische Reaktionen zur Folge, die der Ungleichbehandlung ökonomischer Aktivitäten entgegenwirken. Die Kapitalisierung oder Amortisation ist der Prozeß des Abbaus von diskriminierenden Vergünstigungen oder Belastungen durch marktliche oder politisch-ökonomische Anpassungsreaktionen').

\subsubsection{Die Kapitalisierung im Marktprozeß}

Der Vorgang der Rapitalisierung im Marktprozeß und die Determinanten des Ausmaßes der Kompensation des begünstigenden oder belastenden Effekts sollen anhand der in Abb. G.3 dargestellten Konstellationen diskutiert werden.

Abbildung G.3: Marktkapitalisierung

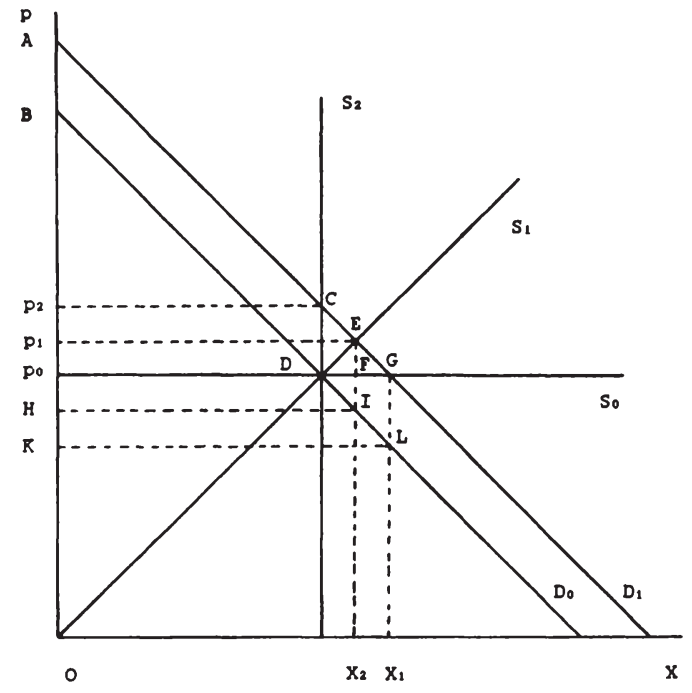

Als ein Beispiel für eine begünstigende Maßnahme kann das Personalkostenzuschußprogramm des Bundes herangezogen werden $^{2}$ ). Rleine und mittlere Unternehmen erhalten einen Zuschuß

1) Vgl. Folkers, C. (1985), S. $284 \mathrm{ff}$

2) Vgl. die Erörterung dieser Problematik am Beispiel des Wohnungsbaus bei Mann, H. (1987), S. 176f; Bittker, B.J. (1975); S. $416 \mathrm{ff}$ und Musgrave, R.A. (1974), S. $347 f f$. 
zu den Kosten für in Forschung und Entwicklung tätiges Personal, da in der Höhe der Personalkosten ein Innovationshemmnis vermutet wirdi').

Eine Subventionierung der Personalkosten entspricht in $\mathrm{Abb}$. G.3 einer Rechtsverschiebung der Nachfragekurve ( $\left.D_{0} \Longrightarrow D_{1}\right)$. Ist das Angebot an entsprechend qualifizierten Arbeitskräften vollkommen elastisch, d.h. es gilt die Angebotskurve So, erfolgt daraufhin eine Ausdehnung der Menge der eingesetzten Arbeitskräfte von $X_{0}$ auf $X_{1}$ bei einem konstanten Preis von po. Der Zuschuß entspricht der Fläche poGLR. Er ist größer als die Produktionskosten der zusätzlichen Menge. Ein Teil des Zuschusses ersetzt somit als Mitnahmeeffekt private Ausgaben.

Ist das Arbeitskräteangebot jedoch relativ unelastisch, dann wird die Nachfrageausdehnung von einer Preiserhöhung (po $\Longrightarrow$ $\left.p_{1}\right)$ begleitet und die Nachfrage wird nur um die Menge $\mathrm{x}_{0} \mathrm{X}_{2}$ erhöht. Von der gesamten subvention $p_{1} E J H$ kommt nur poFJH dem Destinatar $z^{2}$ ).

Eine vollständige Rapitalisierung erfolgt bei völlig unelastischem Arbeitsangebot. Die Preise steigen im vollen Ausmaß der Subventionierung und die an die Nachfrager von Arbeitskräften gerichtete Begünstigung geht voll an die Anbieter über. Das angestrebte Ziel, nämlich eine Erhöhung des Forschungs- und Entwicklungspersonals wird durch die marktliche Kapitalisierung vollständig kompensiert. Struktureffekte werden nicht erzielt und anstelle der innovationswilligen Unternehmen werden die entsprechend qualifizierten Arbeitnehmer begünstigt ${ }^{3}$ ).

\subsubsection{Die Rapitalisierung im politischen Prozeß}

Ebenso wie auf den betroffenen Märkten lösen diskriminierende staatliche Aktivitäten auch im politischen Bereich Reaktionen und Anpassungsprozesse aus. "Wird im Wirtschaftsleben deut-

1) Vgl. Bundesminister für Forschung und Technologie (1986), S. 16ff

2) Vgl. Folkers, C. (1988)

3) Entsprechende Effekte können bei Variationen der Nachfragekapazität abgeleitet werden. 
lich, daß Renten nicht nur durch Teilnahme am Marktgeschehen, sondern auch durch politische Beeinflussungen erreichbar sind, so werden ökonomische Aktivitäten auch in diese Richtung gelenkt."1) Es entwickelt sich eine Ronkurrenz um steuervorteile. Unter Verweis auf andere Industriezweige werden Interessenverbände versuchen, auch für ihren Klientel Begünstigungen $z u$ erhalten ${ }^{2}$ ). Eine Regierung, der es möglich ist, Renten zu erzeugen, wird für die Unternehmen zu einem potentiellen Investitionsobjekt ${ }^{3}$ ). Die durch diese institutionelle Ronstellation verursachte Schwächung des marktlichen Anreizsystems führt zu Veränderungen im Unternehmensverhalten. An die Stelle kreativer Unternehmer treten Lobbyisten'). Statt auf das Aufspüren von Marktchancen wird das Engagement auf unproduktive Umverteilungsprozesse verlagert'). Zur Beeinflussung der politischen Entscheidungsträger werden "Reformkosten und Mehrbelastung ... bis zu dem Punkt eingegangen, an dem ihr Zuwachs dem erwarteten Verteilungsvorteil gleich wird."6) Die Aufwendungen in diesem Proze $\beta$ zehren die individuellen Vorteile auf ("institutionelle Rapitalisierung"7)). Die hier eingesetzten Ressourcen werden aus produktiver Verwendung in marktlichen Aktivitäten abgezogen und in einem unproduktiven Umverteilungswettbewerb verausgabt. Volkswirtschaftlich gesehen werden sie verschwendet und erhöhen die Rosten staatlicher Aktivitäten über die Mehrbelastung hinaus $^{8}$ ). Zudem wird die Rostenkontrollfunktion des Marktes geschwächt, wenn die Unternehmen dann Subventionen erwarten können, wenn aufgrund mangelnder Wettbewerbsfähigkeit Arbeitsplätze gefährdet sind.

1) Folkers, C. (1985), S. 285

2) Vgl. Zeppernick, R. (1987), S. 58

3) Vgl. McKenzie, R.B. (1985), S. 188

4) Vgl. Hiemenz, U./Weiss, F.D. (1984), S. $13 \mathrm{ff}$

5) "In der Tat können in interventionistischen Volkswirtschaften Personen eine industrielle Karriere machen, deren besondere Făhigkeit in der Präsentation vor Regierungsausschüssen liegt und die wissen, wie politische Unterstützung zu organisieren ist. Solche Personen entwickeln jedoch selten neue marktfăhige Produkte." OECD (1984), S. $120 f$

6) Folkers, C. (1983), S. 195

7) Mann, H. (1987), S. 179

8) Vgl. Becker, G.S. (1983), S. $387 f$ und Folkers, C. (1985), S. 286 


\section{$\underline{2.2}$ Die Interventionsakzeleration}

Die Neutralisierung der angestrebten Effekte staatlicher Aktivitäten durch Kapitalisierung bzw. ihre mögliche Ronterkarierung aufgrund unzureichender oder falscher Informationen lösen im politischen Proze $\beta$ weiteren Handlungsbedarf aus. Um die durch diskriminierende Interventionen beabsichtigte Begünstigung bestimmter wirtschaftlicher Aktivitäten fortgesetzt aufrecht zu erhalten, werden ständig neue Eingriffe erzwungen.

Zudem sind die politischen Entscheidungsträger nicht in der Lage, die durch die Kapitalisierung neutralisierten Maßnahmen wieder aufzuheben. Die Interventionen werden zwar durch die Anpassungsreaktionen der Wirtschaftssubjekte wirkungslos, ihre Abschaffung würde jedoch zu Einkommensverlusten bei den nach der Kapitalisierung Begünstigten führen und deshalb auf Widerstand stoßen ${ }^{1}$ ). Die dadurch erfolgende Akkumulation von Eingriffen vermindert die Transparenz, erhöht Informationsund Entscheidungskosten und erschwert die Einschãtzung der Wirkungsweise neuer Maßnahmen.

Neben den durch Kapitalisierung neutralisierten Interventionen verursachen auch die aufgrund von Informations- und Prognosefehlern fehlgeschlagenen Maßnahmen eine wachsende AnzahI von Folgeeingriffen'). Fehleinschätzungen der wirtschaftlichen Entwicklung erzeugen weiteren Interventionsbedarf. So wurden beispielsweise in Fehleinschätzung der Nachfrage- und Weltmarktentwicklung der Ausbau und die Modernisierung der Kapazitäten der europäischen stahlproduzenten staatlich gefördert. Die Folge sind heute massive und in ihrer Intensität zunehmende staatliche Eingriffe zur "stabilisierung" der durch Uberkapazitäten gekennzeichneten Märkte ${ }^{3}$ ).

1) Vgl. Feldstein, M.S. (1976), S. 124 und Tullock, G. (1975), S. 671ff

2) Vgl. Issing, O. (1984), S. $11 \mathrm{ff}$

3) Vgl. HHWA (1986), S. $165 \mathrm{ff}$. Ein weiteres Beispiel ist die Schiffbauindustrie. Hier wurden den Reedern zunächst Subventionen gewährt, um dann später mit Hilfe von Abwrackprämien die geschaffenen Kapazitäten wieder abzubauen; vgl. Rother, D. (1985), S. $131 \mathrm{ff}$ und Kappel, R. (1985), S. 155ff. 
Ein weiterer, insbesondere für die Forschungs- und Technologieförderung bedeutsamer Aspekt, der zu einer Verstärkung staatlicher Aktivitäten führt, ist der Interventionswettbewerb. Die Wirtschaftsföderung, sei es durch steuerliche Vergünstigungen, Subventionen oder sonstige Maßnahmen, hat speziell im Bereich der Technologiepolitik ein positives Image' '. Entsprechend versuchen Politiker in Kommunen und Ländern, sich ein entsprechendes Profil aufzubauen. Da die Aktionen in allen Gebietskörperschaften in die gleiche Richtung gehen und jede Körperschaft versucht, Maßnahmen ihrer "Konkurrenten" zu kontern, kommt es zu einer immer stärkeren subventionierung einzelner Bereiche, die in allen Gebietskörperschaften übereinstimmen, mit zwangsläufig wachsender Diskriminierung von Marktfeldern, die von den politischen Entscheidungsträgern als nicht zukunftsträchtig eingestuft werden ${ }^{2}$ ).

Kapitalisierung, mangelnde Zielerreichung staatlicher Maßnahmen und Interventions- oder Förderwettbewerb führen somit im politischen Prozeß zu einer Interventionsakzeleration ${ }^{3}$. Die Folge sind aufgrund des Beharrungsvermögens früherer Eingriffe wachsende Intransparenz, zunehmende Informationsprobleme bei späteren Entscheidungen und eine massive störung marktlicher Anreize.

\section{MOGLICHREITEN DER INNOVATIONSPOLITIR}

Die Diskussion der notwendigen und der hinreichenden Bedingung ergibt relativ enge Grenzen für eine erfolgreiche staatliche Innovationspolitik. Eine induzierend, d.h. veranlassend gedachte Politik, die bestimmte Innovationsaktivitäten der privaten Wirtschaftssubjekte fördert, ist praktisch unmöglich. Grund dafür ist der Charakter der im Wirtschaftsprozeß

1) Interventionen in diesem Bereich haben zudem den Vorteil, daß mit der Ankündigung mittel- und langfristiger Erfolge einer kurzfristigen Erfolgskontrolle ausgewichen werden kann; vgl. Staudt, E. (1987/88), S. 94.

2) Vgl. Staudt, E. $(1987 / 88)$, S. $93 \mathrm{ff}$

3) Vgl. dazu Folkers, C. (1978) in bezug auf Steuerreformprozesse 
auftretenden strukturen. Während in den exakten Naturwissenschaften Informationen über die relativen Häufigkeiten einzelner Elemente bzw. die Wahrscheinlichkeiten ihres Auftretens ausreichend sind, um bestimmte Ergebnisse abzuleiten, können Aussagen über bestimmte Ergebnisse marktlicher Prozesse nur bei Kenntnis der strukturbildenden Elemente und der Art und Weise, in der sie miteinander verbunden sind, gemacht werden. Es handelt sich um "Erscheinungen organisierter Romplexität", die vollständige Informationen über jedes Element erfordern, wenn Voraussagen über bestimmte Ereignisse abgeleitet werden sollen'l). Da aber fast jedes Ereignis Auswirkungen auf die zukünftigen Entscheidungen eines wirtschaftssubjekts hat' ${ }^{2}$ und zudem Reaktionen auf Innovationen, d.h. neue, bisher nicht dagewesene Erscheinungen a priori nicht bekannt sein können, fehlen einer induzierenden Innovationspolitik die Grundlagen. "Aus diesem Grunde ist die ökonomische Theorie auf die Beschreibung von Arten von Mustern beschränkt, die auftreten, wenn gewisse allgemeine Bedingungen erfüllt sind"3). Die Renntnis dieser Muster oder strukturen') ist nützlich, "um Bedingungen zu schaffen, die förderlich sind, Ergebnisse bestimmter Art hervorzurufen"').

Aufgrund dieser Beurteilung der Möglichkeiten von zielgerichteten Eingriffen in marktliche Prozesse ergibt sich als Aufgabe und ziel der staatlichen Wirtschaftspolitik, Bedingungen zu schaffen, die innovatorische Aktivitäten der wirtschaftssubjekte ermöglichen und Raum für die Entfaltung innovatorischer Prozesse gewähren'). Die Grundzüge einer solchen "akkommodierenden Innovationspolitik"7) werden im folgenden entwickelt.

1) Vgl. Hayek, F.A. von (1975), S. $14 f$

2) Vgl. Hayek, F.A. von (1972), S. 25

3) Hayek, F.A. von (1972), S. 27

4) Der von Hayek im englischen Original verwendete Begriff "pattern" wurde mit seinem Einverständnis zunächst mit Muster, später jedoch mit Struktur übersetzt; vgl. Hayek, F.A. von (1975), FN 3.

5) Hayek, F.A. von (1972), S. $24 f$

6) Bildhaft formuliert Hayek: Aufgabe des Staates ist es nicht, "Ergebnisse zu formen wie der Handwerker sein Werk formt, sondern ein Wachsen zu kultivieren, indem er die geeignete Umgebung schafft, wie es der Gärtner für seine Pflanzen macht"; Hayek, F.A. von (1975), S. 21.

7) Streit, M.E. (1984), S. 35 
In diesem Abschnitt werden die Konsequenzen für innovationspolitische Aktivitäten aus den Ergebnissen dieser Arbeit gezogen. Zunächst wird auf die Funktionen der Unternehmen und des staates in einer marktwirtschaftlichen ordnung eingegangen. Anschließend werden die Grundzüge einer akkommodierenden Innovationspolitik dargestellt.

1. Die Funktionen von Unternehmen und stat in einer marktwirtschaftlichen ordnung

Um die Aufgaben von Unternehmen und Staat im wirtschaftlichen Geschehen zu bestimmen, ist der Ablauf ökonomischer Prozesse in einem marktwirtschaftlichen system zu analysieren. Ausgangspunkt der folgenden Darstellung ist ein dynamisches, evolutorisches system im Sinne der Austrian Economics. In ihm ist ein Gleichgewicht dadurch charakterisiert, daß die individuellen Wirtschaftspläne kompatibel sind'). Bestehen Koordinationslücken zwischen den individuellen Plänen, werden diese im Marktprozeß in Gewinnmöglichkeiten umgesetzt2). Aufgabe der Unternehmen ist es, die "coordinate gaps"3) aufzuspüren und auszugleichen. Im Marktprozeß lassen sich zwei Entdeckungsaufgaben unterscheiden').

Zum einen besteht eine zeitpunktbezogene Koordinationslük$\left.\mathrm{ke}^{5}\right)$. Aufgrund unzureichender Transparenz werden auf einem Markt zum gleichen zeitpunkt an verschiedenen orten die gleichen Transaktionen $z u$ unterschiedlichen Preisen abgewickelt. Aus diesen Preisdivergenzen ergeben sich Gewinnerzielungsmöglichkeiten durch Arbitrageaktivitäten. Wären dies die einzigen Koordinationslücken, so würde sich nach dem Abschluß der Arbitragegeschäfte ein stationäres Gleichgewicht einstellen.

1) Vgl. Hayek, F.A. von (1937), S. $37 f$

2) Vgl. Kirzner, I.M. (1982), S. 141

3) O'Driscoll, G.P.jr/Rizzo, M.J. (1985), S. 6

4) Vgl. Kirzner, I.M. (1982), S. $140 f f$ und Oberender, P. (1987), S. $10 f f$

5) Vgl. Rirzner, I.M. (1982), S. $151 \mathrm{ff}$ 
Zusätzlich bestehen jedoch zeitraumbezogene Abstimmungsmänge ${ }^{1}$ '. Die individuellen Wirtschaftspläne sind in die zukunft gerichtet. In ihnen werden aufgrund der individuellen Erfahrungen und Fähigkeiten unterschiedliche Vorstellungen über die zukunft entwickelt. Um die in den wirtschaftsplänen enthaltenen unterschiedlichen Ansprüche zum Ausgleich zu bringen, ist es Aufgabe der Unternehmen, durch Innovationen die zeitraumbezogenen Koordinationslücken zu schließen. Gewinnerzielungsmöglichkeiten bestehen in diesem Bereich durch die Realisierung von Projekten, welche die Befriedigung bisher nicht erfüllter Bedürfnisse der Konsumenten ermöglichen. Durch die Innovationstätigkeit werden nun ihrerseits wieder Divergenzen geschaffen und neue Arbitragemöglichkeiten eröffnet.

Der Marktprozeß in einer dynamischen Wirtschaft stellt sich somit als eine Abfolge von vor- und nachstoßenden Wettbewerbsaktionen dar, d.h. von Innovationsaktivitäten der Schumpeterschen Pionierunternehmer und von Arbitrageaktivitäten der Misesschen "findigen Unternehmer" 2 '.

Aufgabe des staates in einem marktwirtschaftlichen system ist es, die Anreize und Möglichkeiten zur Entfaltung dieser Aktivitäten zu gewährleisten und ihnen entgegenstehende Hemmnisse zu beseitigen.

\section{2.} Grundzüge einer akkommodierenden Innovationspolitik

Innovationstätigkeit umfaßt die Gesamtheit aller in die zukunft gerichteten Aktivitäten der Unternehmen zur Roordination ihrer Angebotsprogramme mit der in den individuellen wirtschaftsplänen der Konsumenten geäußerten Nachfrage. Die Planabstimmung erfolgt durch neuartige Produkte und Produktqualitäten, durch neue Produktionsverfahren und organisationsformen.

1) Vgl. Kirzner, M.I. (1982), S. $147 \mathrm{ff}$

2) Vgl. Kirzner, I.M. (1973), S. 74 und S. 84ff, der das Konzept von Mises aufnimmt; für die deutsche Ubersetzung vgl. Kirzner, I.M. (1978), S. 59 
Wie in dieser Arbeit gezeigt, scheitert eine induzierende Innovationspolitik an den mit ihr verbundenen Informationserfordernissen. Das für eine solche Politik notwendige Lenkungswissen kann von den wirtschaftspolitischen Entscheidungsträgern nicht erworben werden. Dies betrifft zum einen den Informationsbedarf für Interventionen des staates in den Marktproze $\beta$. Um zielgerichtete Eingriffe vornehmen zu können, ist die Renntnis der Angebots- und Nachfrageverhältnisse des betrachteten Markts und der mit ihm über Romplementaritätsund Substitutionsbeziehungen verbundenen Güter- und Faktormärkte erforderlich. Eine hinreichend genaue Erhebung und Verarbeitung dieser Daten ist im Regelfall praktisch unmöglich. Logisch unmöglich ist zum zweiten eine gezielte Innovationsförderung. Staatliche Förderung wirtschaftlicher Aktivitäten der privaten Wirtschaftssubjekte benötigt Fördergrundlagen, nach denen die Förderungsleistungen bestimmt werden. Innovationen sind jedoch neuartige wirtschaftliche Rombinationen und kōnnen deshalb ex definitione nicht bekannt sein, sondern müssen im Marktproze $\beta$ über Trial-and-Error herausgefunden werden. Eine induzierende Innovationspolitik ist somit im Hayekschen Sinne eine "Anmaßung von Wissen"1) und ökonomisch nicht vertretbar.

Wie die Analyse zeigt, ist es aber möglich, Mustervoraussagen zu treffen. Dies sind Aussagen über die struktur von Prozessen. Die Ausführungen über die Arbitrage- und Innovationsprozesse in dynamischen marktwirtschaftlichen Systemen stellen solche strukturbeschreibungen dar. Aus der Mustervoraussage über den Innovationsproze $\beta$ wird nun unter Verwertung der Ergebnisse dieser Arbeit das Konzept einer akkommodierenden Innovationspolitik entwickelt.

Innovationstätigkeit ist in die zukunft gerichtet. Somit ist mit ihr wie mit allen über mehrere Planungsperioden hinwegreichenden Aktivitäten Unsicherheit über die zukünftigen Umweltzustände verbunden. Das Risiko eines Fehlschlags ist von den Wirtschaftssubjekten $z u$ tragen, welche die Realisierung

1) Hayek, F.A. von (1975), S. 12 
des Innovationsprojekts planen. Aufgabe einer akkommodierenden Innovationspolitik ist es, zielkonforme Anreize zu schaffen, damit die Wirtschaftssubjekte veranlaßt werden, dieses Risiko auf sich zu nehmen, die Institutionen dafür bereitzustellen, daß risikobereite Unternehmer fähig werden, das Innovationsrisiko zu tragen und dafür zu sorgen, daß dieses Risiko nicht durch staatliche Aktivitäten und Reglementierungen zusätzlich erhöht wird.

Im Gegensatz zur induzierenden Politik stellt die akkommodierende Innovationspolitik somit nicht auf bestimmte projekte oder Ergebnisse ab. Innovationen sind neuartig und können deshalb im vorhinein nicht bekannt sein. Deshalb ist der institutionelle Rahmen, in dem Innovationsprozesse ablaufen, Gegenstand der Politik. Ziel der akkommodierenden Innovationspolitik ist es, Anreize für die Suche nach neuartigen wirtschaftlichen Kombinationen zu setzen und Hindernisse für Innovationsaktivitäten $z u$ beseitigen.

Der entscheidende Anreiz zur Ubernahme wirtschaftlichen Risikos ist der Gewinn, der bei zutreffender Einschätzung der zukünftigen Marktentwicklung erzielt werden kann. Ziel der akkommodierenden Innovationspolitik ist es deshalb, Institutionen zur Verfügung zu stellen, die es dem Innovator ermöglichen, sich die Erträge seiner Aktivitäten anzueignen. Das für diesen Zweck adäquate Instrumentarium ist der gewerbliche Rechtschutz. Seine struktur muß so flexibel ausgestaltet werden, daß es möglich ist, auch neue Entwicklungen zu erfassen. Unter diesem Aspekt ist vor allem die Dauer von Patenterteilungsverfahren $z u$ überprüfen. So wurden in den siebziger Jahren nur die Hälte aller Patente in einem Zeitraum von fünf Jahren seit ihrer Anmeldung erteilt').

Zusätzlich sind Ansprüche von Dritten zu überprüfen, die den Gewinnanreiz insbesondere im Vergleich $z u$ alternativen Handlungsmöglichkeiten reduzieren. In diesem Zusammenhang zu nennen sind die absolute Höhe der steuerlichen Belastung von Gewinnen, die - auch steuerliche - Diskriminierung unternehmerischer und risikobehafteter Tätigkeit sowie die arbeits- und

1) Vgl. Gutberlet, K.-L. (1984), S. $18 \mathrm{ff}$ 
sozialrechtlichen Regelungen'), die das Beschäftigungsrisiko teilweise auf den Unternehmer überwälzen und dadurch zum einen das Risiko seiner wirtschaftlichen Aktivitäten erhöhen und zudem das Gewinnpotential und die Risikoprämie reduzieren. D.h. die Kosten der Solidargemeinschaft werden zu unternehmensspezifischen Kosten. Dies reduziert die Anreize für unternehmerische Aktivitäten.

Die Fähigkeit eines Wirtschaftssubjekts, Risiken zu übernehmen, wird durch die Ressourcen bestimmt, die ihm zur Realisierung seiner Innovationsprojekte zur Verfügung stehen. Gegenstand einer akkommodierenden Innovationspolitik sind unter diesem Aspekt die Hochschul- und Ausbildungspolitik, die Rapitalmarktpolitik und die Arbeitsmarktpolitik.

Ober die Hochschul- und Ausbildungspolitik können die Fãhigkeiten der Wirtschaftssubjekte beeinflußt werden, Innovationsmöglichkeiten aufzufinden und $z u$ realisieren. Für die Ausnutzung wirtschaftlicher opportunitäten ist es wichtig, das vorhandene wissen auf neue Bereiche übertragen und die erworbenen Fähigkeiten auch in fremden Anwendungsgebieten einsetzen zu können. Wichtig ist somit nicht das Ausmaß, sondern die struktur der Ausbildung. Entscheidend ist die Vermittlung instrumenteller Fähigkeiten, um die Wirtschaftssubjekte $z u$ innovativem Denken zu befähigen. Je flexibler ein potentieller Unternehmer $z u$ reagieren in der Lage ist, desto besser kann er ein gegebenes Risiko einschätzen und mit ihm umgehen. Eine analytische Fähigkeiten betonende und Transferwissen vermittelnde Hochschul- und Ausbildungspolitik erleichtert es den Wirtschaftssubjekten, neuartige wirtschaftliche Konstellationen einzuschätzen und verbessert ihre Fähigkeiten, das mit ihrer Bewältigung verbundene Risiko auch einzugehen.

Neben den persönlichen Fertigkeiten bestimmen auch die Möglichkeiten der Finanzierung risikobehafteter, innovativer Projekte die Fähigkeit, Risiken zu übernehmen. Ziel einer akkommodierenden Innovationspolitik muß es deshalb sein, Regelungen, die zu Fehllenkungen von Risikokapital führen und Institutionen, die den zutritt zu Risikokapitalmärkten be-

1) Vgl. die Ubersicht bei Watrin, C. (1984), S. $336 f$ 
schränken, zu beseitigen. Angesichts der spekulativen Investitionen in hochriskante Abschreibungsprojekte kann von einem Mangel an Risikokapital nicht ausgegangen werden. Vielmehr sind beträchtliche Kapitalfehllenkungen in den Wohnungsbau, in Explorationsunternehmen, in Abschreibungsgesellschaften, "Zahnärzteschiffe", etc. zu konstatieren. Zusätzlich wird der Zugang zum Rapitalmarkt für risikobehaftete kleine und mittlere Unternehmen blockiert. Als innovationspolitische Aufgabe ist deshalb die Beseitigung von Anreizstrukturen, die zu gesamtwirtschaftlich schädlichen Rapitalfehllenkungen führen') und eine offnung der Rapitalmärkte für die Finanzierung überdurchschnittlich risikobehafteter Projekte bzw. Unternehmen zu sehen.

"Die außerordentliche Unstabilităt der experimentierenden Wirtschaftspolitik - so der währungs-, Handels-, steuer- und Lohn-Politik -, wie sie während der letzten Jahrzehnte in den meisten Industrieländern bestand, hat das Element der Unsicherheit wesentlich vergrößert."2) Wie bereits in Kapitel $E$ angesprochen, erhöhen die Kurzlebigkeit und die Unklarheit gesetzlicher Regelungen sowie der Aktionismus bei wirtschaftspolitischen Eingriffen die streuung der für die Innovationsprojekte relevanten Plandaten. Bei risikoaversem Verhalten führt dies selbst bei einer Ronstanz des Erwartungswerts zu einem Rūckgang der Innovationsanstrengungen. Gleichzeitig werden auf die Unternehmen durch zahlreiche arbeitsund sozialrechtliche Bestimmungen zusätzliche Rosten überwälzt. Dadurch wird bei gegebenem Risiko die Risikoprämie reduziert und die Risikoübernahmebereitschaft negativ beeinflußt. Die akkommodierende Innovationspolitik hat deshalb für "eine gewisse Konstanz der Daten"3) zu sorgen, damit längerfristige Projekte nicht wegen des wirtschaftspolitischen Risikos zugunsten kurzfristiger unternehmerischer Aktivitäten vernachlässigt werden'). Dieses Ziel kann mit Hilfe konstitutioneller Regeln erreicht werden, da diese nur mit qualifi-

1) Vgl. dazu das Beispiel bei Schneider, D. (1980), S. $285 f$

2) Eucken, H. (1955), S. $287 f$

3) Eucken, H. (1955), S. 288, Hervorhebung im Original

4) Vgl. Streit, M.E. (1984), S. 45 
zierten Mehrheiten zu ändern sind und deshalb im politischen Prozeß "quasi-permanent" gültig sind1).

Als zweites Merkmal der Innovationstätigkeit ist $z$ u betonen, daß die zukunftsgerichtete Plankoordination durch die wirtschaftliche Verwertung neuer Produkte und Produktqualitäten, neuartiger Produktionsverfahren und organisationsformen bzw. allgemein durch neuartige Kombinationen von Nachfrage und angebotenen Mitteln zu ihrer Befriedigung erreicht wird. Als Aufgabe einer akkommodierenden Innovationspolitik resultiert daraus, daß insbesondere im Rahmen der Wettbewerbs- und Handelspolitik die offnung und offenhaltung des wirtschaftlichen und gesellschaftlichen systems für Neuerungen zu sichern ist. Diese Forderung betrifft zum einen die offnung von Märkten für neue Wettbewerber und zum zweiten, daß gesetzliche Regelungen derart auszugestalten sind, daß die Einführung neuartiger Lösungen für wirtschaftliche Probleme nicht ausgeschlossen wird. In diesem sinne sind Marktzutrittsregulierungen, durch kartellähnliche zusammenschlüsse gesetzte Normen und Konditionen sowie außertarifäre Handelshemmnisse zu überprüfen und die Möglichkeiten der selbstregulierung einzelner Branchen zu reduzieren²'.

\section{ZUSAMMENFASSUNG}

Gegenstand dieses Kapitels war die Analyse der Möglichkeiten und Grenzen innovationspolitischer Aktivitäten staatlicher Träger der Wirtschaftspolitik. Als Voraussetzungen innovationspolitisch motivierter Maßnahmen wurden notwendige und hinreichende Bedingungen für staatliche Eingriffe in ein marktwirtschaftliches system diskutiert. Die für staatliche Maßnahmen erforderlichen Informationen sowie die durch solche Aktivitäten ausgelösten Rapitalisierungsprozesse beschränken die Möglichkeiten einer induzierend angelegten wirtschaftspolitik.

1) Vgl. Buchanan, J.M. (1976), S. 27

2) Diese Forderung wird in den Gutachten des Sachverstăndigenrates regelmäßig erhoben; vgl. Sachverständigenrat (1986) und (1985). 
Zudem bestehen innovationsspezifische Grenzen für solche Eingriffe. Innovationen sind Aktivitäten zur wirtschaftichen Verwertung neuer Produkte und Produktqualitäten sowie neuartiger Produktionsverfahren und Organisationsformen. Eine gezielte Förderpolitik setzt jedoch die Kenntnis des Fördergegenstandes voraus. Da Innovationen a priori unbekannt sind, ist eine induzierend angelegte Innovationspolitik von der $\mathrm{Na}-$ tur der sache her nicht möglich. Deshalb wurde eine strategie für eine akkommodierende Innovationspolitik vorgeschlagen. Dieses Konzept basiert auf dem Modell einer dynamischen Wirtschaft. Innovations- und Arbitrageaktivitäten füllen die Roordinationslücken zwischen den individuellen planungen der Wirtschaftssubjekte. Um Innovationsaktivitäten $z u$ fördern, sind deshalb im Rahmen einer akkommodierenden Innovationspolitik Diskriminierungen risikobehafteter, unternehmerischer Aktivitäten zu beseitigen, Erhöhungen des unternehmerischen Risikos durch wirtschaftspolitische Maßnahmen zu verhindern, sowie Kapital- und Gütermärkte für neuartige Vorhaben offenzuhalten.

Im folgenden wird die Innovationspolitik in der Bundesrepublik Deutschland unter Bezug auf die Ergebnisse dieser Arbeit kritisch analysiert. Zunächst werden ziele und Begründungen für innovationspolitische Maßnahmen des staates untersucht. Anschließend erfolgt eine Diskussion der aktuellen Aktivitäten zur Innovationsförderung in der Bundesrepublik Deutschland. Der gegenwärtigen, induzierend angelegten Politik werden Vorschläge für Maßnahmen im Rahmen einer akkommodierenden Innovationspolitik gegenübergestellt. 
$\underline{H}$. INNOVATIONSPOLITIR IN DER BUNDESREPUBLIR DEUTSCHLAND

$\underline{I}$ ZIELE UND GRUNDSATZE

"Die deutsche Wirtschaftspolitik zielt darauf ab, die ordnungspolitischen Grundlagen für eine aus sich heraus dynamische, anpassungs- und innovationsfähige Wirtschaft $z u$ schaffen."1) Sie ist "auf die stärkung der endogenen Wachstumskräfte des Marktes gerichtet."2)

Ober Innovationen sollen Produktivitätssteigerungen erzielt, Investitionen induziert und dadurch Arbeitsplätze geschaffen werden ${ }^{3}$ ). Der Innovationsbegriff wird hier eng gefaßt und umschließt nur "die erfolgreiche Umsetzung wissenschaftichtechnischer Erkenntnisse in marktfähige technologische Produkte und Verfahren"4).

Wird diese zielformulierung durch die Grundsatzüberlegungen ergänzt, welche die Bundesregierung nach eigener Aussage bei der Entscheidung über Fördermaßnahmen leiten'), so ergeben sich zunächst keine Anhaltspunkte für die Notwendigkeit spezifisch innovationspolitischer Aktivitäten:

"In einer marktwirtschaftlichen ordnung ist industrielle Forschung, Entwicklung und Innovation originäre Aufgabe der Unternehmen. ... statiche Forschungs - und Technologiepolitik darf nicht ... die Produktionsstruktur der Wirtschaft in bestimmte Bahnen lenken wollen."6)

"Eine auch vom ordnungspolitischen Grundverständnis konsequent marktwirtschaftich orientierte Politik, die für günstige und verläßliche wirtschafts-, gesellschafts- und forschungspolitische Rahmenbedingungen sorgt, die Vertrauen begründet und die Eigenverantwortung stärkt, ist notwendige

1) Presse- und Informationsamt der Bundesregierung (1987), S. 1

2) Presse- und Informationsamt der Bundesregierung (1987), S. 1

3) Vgl. Bundesregierung (1983), S. 12 sowie Bundesminister für Forschung und Technologie (1984), S. 34 und $38 f$

4) Bundesministerium der Finanzen (1985), S. 41

5) Vgl. Bundesregierung (1983), S. 3

6) Bundesminister für Forschung und Technologie (1984), S. 40 
Voraussetzung und zugleich der stärkste Antrieb für Innovation in der Wirtschaft."1)

In dieser Sichtweise gibt es keinen Raum für induzierend angelegte Interventionen, die über eine ordnungspolitisch ausgerichtete, akkommodierende Innovationspolitik hinausgehen. Die Bundesregierung beschränkt sich jedoch nicht auf diese "ordnungspolitische Denkrichtung"2), sondern sieht einen Handlungsbedarf über die setzung und Verbesserung von Rahmenbedingungen hinaus.

"Forschungs- und Technologiepolitik ist auch dort gefordert, 'wo aus übergeordneten gesellschaftlichen oder gesamtwirtschaftichen Gründen Forschung und Entwicklung einer Unterstützung bedürfen. Dies gilt

- vor allem für die Grundlagenforschung und für Aufgaben der staatlichen Daseins- und Zukunftsvorsorge (z.B. Sicherheits-, Umwelt- und Gesundheitsforschung);

- für besonders risikoreiche, aufwendige, die Privatwirtschaft überfordernde längerfristige Forschung und Entwicklung (wie z.B. in der Rernenergie oder der Luft- und Raumfahrt), oder in besonders wichtigen branchenübergreifenden Schlüsseltechnologien (z.B. Informationstechnologie)." "3)

Ein weiteres Argument, das im Forschungsbericht 1984 nicht explizit auftaucht, begründet staatliche Interventionen mit ähnlichen Maßnahmen anderer Länder') bzw. mit der Position der Bundesrepublik Deutschland im internationalen Wettbewerb auf dem Hochtechnologiesektor's).

Im folgenden wird geprüft, inwieweit diese Argumente aus ökonomischer sicht über ordnungspolitische Maßnahmen hinausgehende Aktivitäten begründen kōnnen.

1) Bundesregierung (1983) S. 4; vgl. auch S. $14 f$

2) Maas, C. (1986), S. 10

3) Bundesminister für Forschung und Technologie (1984), S. 44; 2itat im Original: Jahreswirtschaftsbericht der Bundesregierung (1984), S. 14

4) Vgl. Bundesminister für Wissenschaftliche Forschung (1969), S. 86

5) Vgl. Bundesregierung (1983), S. 7 und 9 sowie Bundesminister für Forschung und Technologie (1984), S. 33 
Notwendige Bedingung für Eingriffe des staates in Marktprozesse ist das Vorliegen von Marktversagen. D.h. der Markt ist aufgrund von Unvollkommenheiten nicht in der Lage, die ihm übertragenen Aufgaben zu erfüllen. In bezug auf innovationspolitisch motivierte staatliche Interventionen bedeutet dies, "daß der Anreiz für innovative Aktivitäten zu gering ist"1) und deshalb gesamtwirtschaftlich gewünchte Aktivitäten unterbleiben bzw. nur in suboptimalem Ausmaß erfolgen.

1. Externalitäten

Die anreizmindernde wirkung externer Effekte beruht darauf, daß der Innovator sich nicht die gesamten Erträge seiner Aktivitäten aneignen kann. Da der Innovator den nicht appropriierbaren Teil der Erträge seiner Anstrengungen in seinem Ralkül nicht berücksichtigt, sinkt das Ausmaß seiner Aktivitäten unter das gesamtwirtschaftlich erwünschte Niveau. Zum einen nimmt er einzelne Projekte überhaupt nicht in Angriff, zum anderen reduziert er Umfang oder Tempo seiner Anstrengungen.

\subsection{Grundlagenforschung}

Die Grundlagenforschung umfaßt Aktivitäten, die auf die Erweiterung des grundlegenden wissenschaftlichen Erkenntnisstandes ausgerichtet sind. Ihre Ergebnisse sind typischerweise nicht direkt wirtschaftlich nutzbar ${ }^{2}$. Sie bilden die Basis für weitere Anstrengungen insbesondere im Bereich der angewandten Forschung.

Da Grundlagenwissen in der Regel keine gewerblich verwertbaren technischen Lösungsmöglichkeiten verkörpert, sondern "nur" aus Informationen besteht, ist es nicht patentierbar. Zudem können seine externen Effekte auch durch Verkauf nicht

1) Maas, C. (1986), S. 11

2) Vgl. Paulenz, R. (1978), S. 28 
internalisiert werden, da Informationen den Charakter eines offentlichen Gutes haben'). Wird versucht, durch Geheimhaltung die Erträge des Erkenntnisfortschritts zu internalisieren, so treten ebenfalls Ineffizienzen auf, da der Innovator die Verbesserung des Grundlagenwissens in der Regel nur in Teilbereichen nutzen kann'). Außerdem wird ineffiziente Parallelforschung nicht durch Veröffentlichung der Ergebnisse verhindert.

Erfolgt die Allokation der Ressourcen aufgrund einzelwirtschaftlicher Kalküle, so ist für den Bereich der Grundlagenforschung $z u$ erwarten, daß wegen der externen Effekte $z u$ wenig Mittel in diese Verwendung gelenkt werden. Dies schließt allerdings nicht aus, daß in einzelnen Bereichen auch für private Wirtschaftssubjekte genügend (Gewinn-)Anreize bestehen, um Grundlagenforschung zu betreiben ${ }^{3}$ ).

staatliche Förderung der Grundlagenforschung ist ökonomisch begründet. Dabei ist jedoch darauf zu achten, daß die Praxis der Mittelvergabe nicht zu einer Lenkung der Anstrengungen in eine bestimmte Richtung führt, sondern daß auch in diesem Bereich die steuerfunktion dem Wettbewerb im Sinne einer Konkurrenz der Forschungsinstitutionen überlassen bleibta).

\subsection{Angewandte Forschung und Entwicklung}

Angewandte Forschung zielt auf praktisch verwendbare Ergebnisse, die in der Entwicklung zu Produktions-bzw. Marktreife geführt werden's).

Kann das vom Innovator bei diesen anwendungsbezogenen Aktivitäten generierte wissen von Dritten genutzt werden, so sind diese in der Lage, einen Teil des durch dieses wissen ent-

1) Informationen sind unteilbar und nahezu kostenlos reproduzierbar; vgl. Arrow, K.J. (1971a), S. $147 f$.

2) Vgl. Paulenz, R. (1978), S. 79

3) Vgl. zum Beispiel die von Technologieunternehmen betriebenen Forschungslaboratorien

4) Vgl. dazu auch Bundesminister für Forschung und Technologie (1984), S. $18 \mathrm{f}$

5) Vgl. Paulenz, R. (1978), S. $29 f$ 
standenen Gewinnpotentials abzuschöpfen und den Innovator am Markt eventuell sogar zu unterbieten, da sie die Kosten der Forschungs- und Entwicklungstätigkeit nicht amortisieren müs$\operatorname{sen}^{1}$ ).

Gegen ein solches Marktversagen lassen sich jedoch mehrere Argumente anführen.

Zum einen stehen geeignete Instrumente zur Verfügung, um die externen Effekte zu internalisieren. Gewerblich verwertbare, neuartige Lōsungen technischer Probleme sind patentierbar. Die Ergebnisse angewandter Forschung und Entwicklung besitzen regelmäßig eine hinreichende Marktnähe, um diese Bedingung für die Patentierbarkeit zu erfüllen. Sind die Ergebnisse innovativer Aktivitäten nicht-technischer Natur, so läßt sich auch durch die entsprechende Ausgestaltung urheberschutzrechtlicher Bestimmungen') bzw. des sonstigen Gewerblichen Rechtschutzes (Gebrauchsmuster-, Geschmacksmuster- und Warenzeichenrecht) die Internalisierung der externen Effekte der Innovationstätigkeit erreichen ${ }^{3}$ ).

Außerdem wird die Abschöpfung der Innovatorenrente durch Konkurrenten dadurch erschwert, daß zur Imitation Erfahrungen und Fähigkeiten vorhanden sein müssen, die der Innovator im Innovationsproze $\beta$ erworben hat. Das Wissen um die Techniken allein reicht zur Produktion nicht aus. Das ergänzend erforderliche know-how kann nur in einem zeitaufwendigen Trialand-Error-Prozeß gewonnen werden'). Somit verbleibt dem Innovator auch bei unzureichendem Patentschutz ein konkurrenzfreier zeitraum, in dem er sich die Erträge seiner Innovationsanstrengungen aneignen kann. Dieser Zeitraum wird um so größer sein, je aufwendiger die Innovation ist, da parallel zum Anspruchsniveau der Innovation auch die Anforderungen an das Rnow-how wachsen werden.

1) Vgl. Maas, C. (1986), S. 14

2) Z.B. für Computer-Software

3) Zu Gegenstand und Umfang dieser Schutzrechte vgl. Eisenmann, H. (1985)

4) Zur Unterscheidung von "technique" und "logy" vgl. Nelson, R.R. (1982), S. 467 
Die Diskussion der mangelnden Anreizwirkung des Marktsystems wurde bislang auf der Grundlage eines Vergleichs der erwarteten Rosten und Erträge einer Innovation geführt. Werden zusätzlich dynamische Wettbewerbsprozesse in die Argumentation einbezogen, dann wird ein weiterer Mechanismus deutlich, der der Problematik externer Effekte entgegenwirkt.

Um ihre Marktposition zu halten, sind Unternehmen auf innovationsintensiven Märkten ständig gezwungen, Marktentwicklungen nachzuvollziehen. Somit resultiert schon aus dem Konkurrenzdruck auf Wettbewerbsmärkten ein ständiger Zwang zu Forschungs- und Entwicklungsaktivitäten ${ }^{1}$ ). Aus den externen Effekten der Innovationstätigkeit läßt sich somit kein Marktversagen ableiten, da Instrumente zu ihrer zumindest partiellen Internalisierung zur Verfügung stehen. Zudem können sich Dritte die Externalitäten nur begrenzt aneignen. Auch bestehen neben dem Gewinn noch weitere Anreize, die sich aus dem Wettbewerbsprozeß ergeben, die zu einer Forcierung von Innovationsanstrengungen führen.

\section{$\underline{2}$.} Unsicherheit

Je weiter Aktivitäten in die zukunft gerichtet sind, je länger also der Planungszeitraum, desto unsicherer und weniger vorhersehbar sind die zukünftigen Umweltzustände. Da Preise nur "Ex-post-Indikatoren (sind), die günstigstenfalls die Knappheitsrelationen zum Investitionszeitpunkt angeben"2), wird eine dem "marktwirtschaftlichen system inhärente Präferenz für das Bekannte" unterstellt3') und auf ein für die marktwirtschaftliche ordnung "charakteristisches Defizit an vorausschauender Technologieentwicklung"4) geschlossen.

Diese Argumentation basiert auf zwei Annahmen. Zum einen wird unterstellt, daß auf dem Markt nur aktuelles Angebot und aktuelle Nachfrage koordiniert werden. D.h. die Marktabstimmung

1) Vgl. Kapitel D

2) Meißner, H. (1974), S. 15

3) Hauff, V./Scharpf, F.H. (1975), S. 48

4) Hauff, V./Scharpf, F.H. (1975), S. 49 
erfolgt ohne zeitlichen Bezug, sozusagen zeitlos'1. Zum zweiten wird vorausgesetzt, daß der zeitliche Horizont von Marktentscheidungen hinter dem gesamtwirtschaftlich gewünschten zurückbleibt bzw. daß die einzelwirtschaftliche Diskontierungsrate und die in ihr enthaltene Risikoprämie über die gesamtwirtschaftlich als optimal erachtete hinausgeht.

In bezug auf die These von der Kurzsichtigkeit des Marktes ist zunächst festzustellen, daß der Markt tatsächlich aktuelle Nachfrage mit gegenwärtigem Angebot abstimmt. Das heißt aber nicht, daß das Marktergebnis isoliert zeitpunktbezogen ist und in keinem zeitlichen zusammenhang steht. Sowohl Angebots- als auch Nachfrageentscheidungen basieren auf individuellen Wirtschaftsplänen, in denen Erfahrungen der Vergangenheit und in die Zukunft gerichtete Dispositionen miteinander abgestimmt werden. "Prices are inherently future oriented, precisely because every action in the market place ipso facto involves an expectation."2) Insbesondere bei Entscheidungen auf Investitionsgütermärkten und Rapitalmärkten sind solche Langzeitüberlegungen offensichtlich. Das Argument des Marktversagens bei der steuerung zukünftiger Entwicklungen verkennt den zeitlichen zusammenhang, in dem individuelle Angebots - und Nachfrageentscheidungen stehen ${ }^{3}$ ).

Gegen eine "Präferenz für das Bekannte"4) und die daraus folgende Neigung zur Unterlassung zukunftsgerichteter Aktivitäten spricht, daß nie alle Unternehmer ihre Gewinne als ausreichend bzw. ihre Ertragssituation als zufriedenstellend ansehen und deshalb auf innovative Tätigkeiten verzichten werden. Vielmehr wird es immer Unternehmertypen geben, die durch vorstoßende Wettbewerbsaktionen versuchen, zusätzliche (Pionier-) Gewinne zu erzielen').

Die Frage, ob der einzelwirtschaftliche Planungshorizont im vergleich zum gesamtwirtschaftich erwünschten $z u$ kurz ist bzw. ob die Diskontierungsrate der Marktteilnehmer gegenüber der gesamtwirtschaftlichen zu hoch ist, kann nur unter Bezugnahme auf die zur Diskussion stehende alternative Ausgestal-

1) Vgl. Issing, O. $(1979 / 80)$, S. 36

2) O'Driscoll, G.P.jr (1977), S. 27; Hervorhebung im Original

3) Vgl. Helmstădter, E. (1978), S. $18 \mathrm{ff}$ und Issing, 0. (1979/80), S. $36 \mathrm{f}$

4) Hauff, V./Scharpf, F.W. (1975), S. 48

5) Vgl. Schumpeter, J.A. (1964), S. $119 f f$ 
tung beantwortet werden. Daß der Planungshorizont von politischen Entscheidungstrāgern über den der Einzelwirtschaftssubjekte hinausreicht, wird zweifelhaft, wenn die von Wiederwahlüberlegungen bestimmte, kurzfristig taktierende Behandlung auch von Langfristproblemen') im politischen Proze $\beta$ betrachtet wird. Was die Einschätzung des Risikos innovativer Aktivitäten angeht, spricht einiges dafür, den betrieblichen Entscheidungsträgern eine höhere Kompetenz als Politikern zuzuschreiben. Den betrieblichen Entscheidungsträgern ist es eher möglich, die Risiken eines Projekt,s für ihr Unternehmen zu bestimmen, da sie zugang zu betriebsinternen Informationen haben und daraus das Risikopotential für das Unternehmen abschätzen können. Zudem sind sie besser mit den technischen und wirtschaftichen Problemen von Innovationsprojekten vertraut als betriebsexterne Beobachter. Auch haben sie direkten Kontakt zu potentiellen Abnehmern und können deren Nachfragewünsche besser überblicken.

Außerdem besitzen die privaten Wirtschaftssubjekte größere Anreize, sich Informationen über die Risiken ihrer Handlungen zu beschaffen, da sie im Gegensatz zu den Politikern selbst für die Folgen möglicher Fehlschläge haften²).

Zusammenfassend ist festzustellen, daß unter dem Aspekt der Unsicherheit bei zukunftsgerichteten Aktivitäten kaum ein Marktversagen abgeleitet werden kann.

\section{3.} Unteilbarkeiten

Ein Marktversagen wird auch daraus abgeleitet, daß bei bestimmten Projekten "das technisch-wissenschaftliche und wirtschaftliche Risiko hoch ist, der finanzielle Einsatz für die in Frage kommenden Unternehmen $z u$ groß, und auf absehbare

1) Vgl. beispielsweise die Behandlung der Reform im Gesundheitswesen und der Rentenreform. Die hier zu beobachtenden kurzfristigen und aufschiebenden Entscheidungen lassen auf einen recht kurzen Planungshorizont schließen. Dieser resultiert aus der struktur des Wahlaktes der Wăhler, da sie ihre Entscheidungen am Nutzeneinkommen aus der staatlichen Tătigkeit ausrichten; vgl. Downs, A. (1968), S. $37 f f$.

2) Vgl. Maas, C. (1986), S. $51 f$ 
Zeit der Markt die neuen technologischen Lösungen nicht von selbst erbringen wird."1)

Diese Begründung enthält mehrere Argumente. Die Aspekte der Risikoeinschätzung und der Länge des Planungshorizonts privater Wirtschaftssubjekte wurden bereits diskutiert. Hier soll nur auf die Bedeutung von Unteilbarkeiten eingegangen werden. Die organisationsmöglichkeiten in einem marktwirtschaftlichen system sprechen dagegen, daß ökonomisch attraktive projekte von den privaten wirtschaftssubjekten unterlassen werden. Auch wenn der Finanzierungsbedarf die Möglichkeiten eines einzelnen Unternehmens übersteigt, sind Rooperationsformen denkbar und sofern ertragversprechend auch wahrscheinlich, welche die Realisierung solcher Projekte ohne staatliche Interventionen erlauben' ${ }^{2}$.

Gegen das Argument des Marktversagens aufgrund von Unteilbarkeiten sprechen insbesondere auch die Erfahrungen mit der in diesem Zusammenhang immer angeführten Rernenergietechnik ${ }^{3}$ ). Die Entwicklung von zwei der fünf im Rahmen des Eltville-Programms geförderten Reaktortypen wurde bereits vor Anlaufen der staatlichen projektförderung begonnen und während des Förderprogramms wurde von einem Unternehmen ein weiteres Reaktorkonzept vollständig aus eigenen Mitteln entwickelt4).

\section{Förderwettbewerb}

Neben die verschiedenen Formen des Marktversagens treten bei der Begründung der Forschungs- und Technologiepolitik die gleichgerichteten Aktivitäten anderer Industrienationen zur Rechtfertigung staatlicher Maßnahmen. Eigene forschungs- und technologiepolitische Anstrengungen werden als erforderlich angesehen, um "ein technisch hochstehendes, konkurrenzfähiges Güterangebot" zu entwickeln und um "im internationalen Wettbewerb bestehen" zu kōnnen').

1) Bundesminister für Forschung und Technologie (1984), S. $44 f$

2) Vgl: beispielsweise das Projekt zur Untertunnelung des Armelkanals, das privat finanziert wird.

3) Vgl. Bundesminister für Forschung und Technologie (1984), S. 44

4) Vgl. Keck, 0. (1980), S. $312 f f$

5) Bundesregierung (1983), S. 13 und S. 9 
Im folgenden sollen zunächst einige oberlegungen $z u$ den Rosten solcher Interventionen angestellt werden ${ }^{1}$ '. Anschließend werden die Rückwirkungen der Maßnahmen auf den internationalen Handel betrachtet.

Abbildung H.1: Subventionsbedarf bei konstanten stückkosten

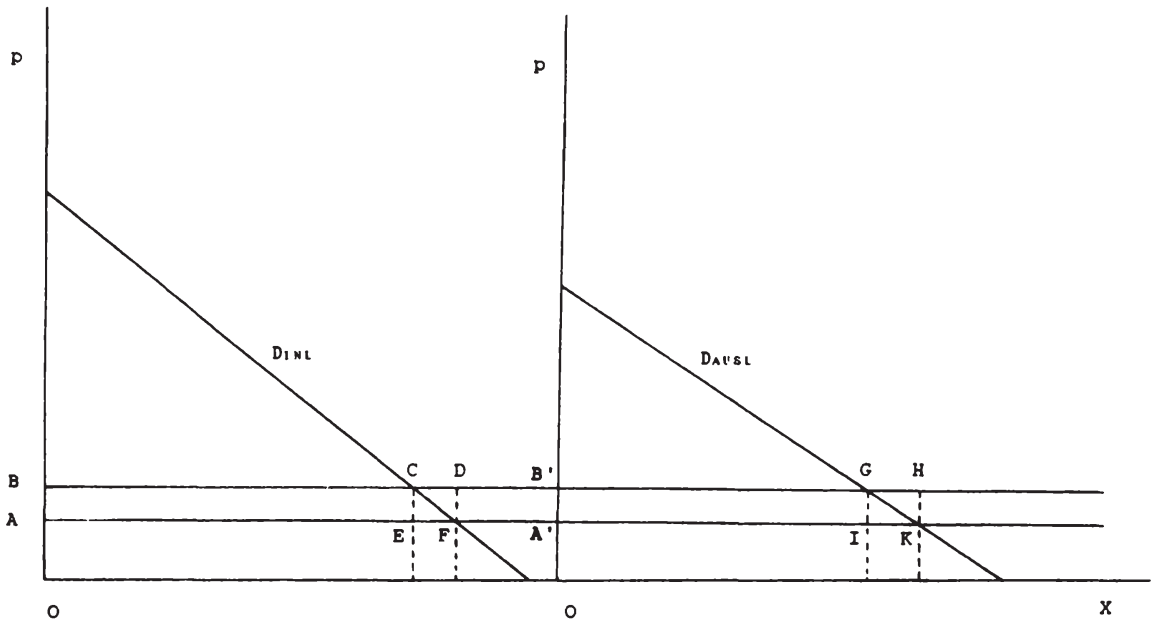

In Abb. H.1 sind die Nachfragebedingungen getrennt für den Inlands- und den Auslandsmarkt dargestellt. Die Produktionsbedingungen sind durch konstante Durchschnittskosten charakterisiert. Eventuelle Forschungssubventionen schlagen sich in einer Parallelverschiebung der Kostenkurve nieder.

Das ausländische Ronkurrenzunternehmen besitze nun einen Kostenvorteil, z.B. durch subventionierung, und sei in der Lage, zu einem Preis von OA anzubieten. Das einheimische Unternehmen könne dagegen nur bei einem Preis größer als OB verlustlos anbieten.

Unter diesen Bedingungen kann das ausländische Unternehmen zum Preis von $O B$ anbieten, ohne seinen Ronkurrenten zum Markteintritt zu veranlassen. Seine (Innovations-) Rente be-

1) Dazu Klodt, H. (1987), S. $8 \mathrm{ff}$ 
trägt auf dem inländischen Markt ABCE und auf dem ausländischen A'B'GI. Durch diese monopolistische Preissetzung entsteht auf den beiden Märkten ein Wohlfahrtsverlust von CEF bzw. GIK.

Soll das einheimische Unternehmen durch eine Subventionierung zum Markterfolg geführt werden, so ergibt sich ein subventionsbedarf von ABDF + A'B'HR, da bei einem höheren Preis als $O A$ das ausländische Unternehmen mit Preissenkungen reagieren kann. Der Wohlfahrtseffekt dieser Maßnahme für das Inland ist negativ. Dem Gewinn in Höhe des vermiedenen Rentenabflusses an das Ausland $A B C E$ und des Harberger-Dreiecks CEF steht der Subventionsaufwand $A B D F+A^{\prime} B^{\prime} H K$ gegenüber. Dieser ist zudem um die Mehrbelastung zu erhöhen, die bei der Erhebung der Mittel zur Finanzierung der subvention verursacht wird. Bei konstanten Durchschnittskosten ist es somit "wenig sinnvoll, einen internationalen subventionswettlauf $z u$ starten oder daran teilzunehmen."1)

Als zweites soll der Fall untersucht werden, in dem die Forschungs- und Entwicklungskosten produktionsunabhängig anfallen und somit Fixkosten der Produktion dieses Gutes darstellen.

In $\mathrm{Abb}$. H.2 sind in- und ausländische Nachfrage aggregiert und der Verlauf der Durchschnittskostenkurve ist bei konstanten Grenzkosten OA eingezeichnet.

Das ausländische Unternehmen (A) sei zuerst auf dem Markt. Ihm unterstellt sein (potentieller) Konkurrent (B) bestimmte Marktstrategien und schätzt demgemã seinen subventionsbedarf ab. Die beiden Unternehmen planen ihr Angebot unter den gleichen Kostenbedingungen. Zwei extreme Verhaltensmuster werden im folgenden betrachtet ${ }^{2}$ ).

Ist das den Markteintritt planende Unternehmen vorsichtig, so unterstellt es dem etablierten Unternehmen, daß es auf Anderungen des gesamten Marktangebots nur mit Preisänderungen reagiert. Der potentielle Ronkurrent geht somit von einer konstanten Outputerwartung aus. Ob bei dieser Annahme über

1) Klodt, H. (1987), S. 11

2) Vgl. Bain, J.S. (1956), S. 97 
das Verhalten des Etablierten ein Markteintritt gewinnbringend ist, kann ermittelt werden, indem die DTR-Kurve des potentiellen Konkurrenten um die Angebotsmenge des Etablierten nach rechts verschoben wird. In Abb. H.2 tangiert die DTKB -Kurve die Nachfragekurve. Bei der vom etablierten Unternehmen A gewählten Preis-Mengen-Kombination ist ein Markteintritt für B weder gewinn- noch verlustbringend'). Nur bei einem Preis höher als pA wäre ein Markteintritt für B erfolgversprechend2).

Abbildung H.2: Subventionsbedarf bei fallenden stũckkosten

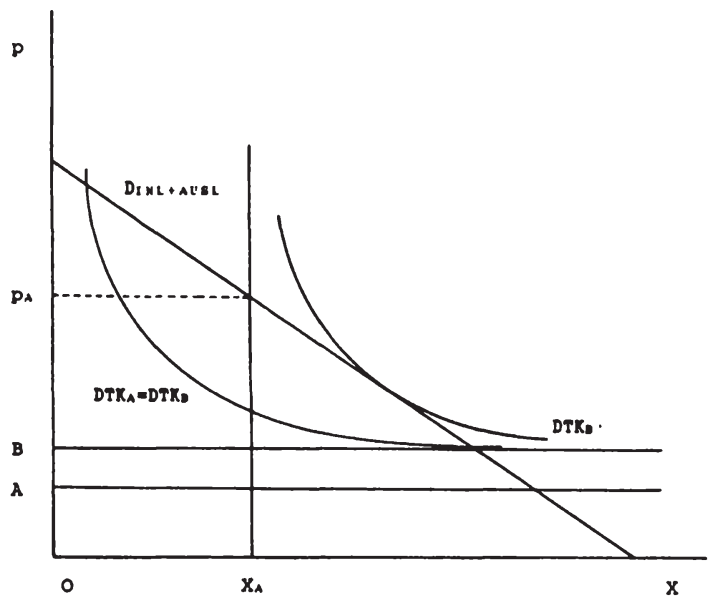

Ist Unternehmen $B$ dagegen aggressiv und unterstellt, daß das etablierte Unternehmen den Preis konstant hät und den bei seinem (B's) Eintritt erfolgenden Absatzrückgang hinnimmt, so kann es Unternehmen A bei jedem Preis oberhalb des kostendekkenden Preises $O B$ bzw. oberhalb von OA, wenn die Fixkosten als sunk costs betrachtet werden, durch Preisunterbietung vom

1) Bei diesen Produktions- und Marktbedingungen kann also auch ohne einen Kostenvorteil eine Innovationsrente abgeschöpt werden.

2) Vgl. Scherer, F.M. (1980), S. $332 \mathrm{ff}$ 
Markt verdrängen. Der Subventionsbedarf, der bei gleichen Kostenverläufen der Ronkurrenten zur Verdrängung des etablierten Unternehmens erforderlich ist, hängt davon $a b$, wie vom etablierten Unternehmen die Fixkosten in der Preiskalkulation behandelt werden. Werden sie als sunk costs betrachtet, so muß Unternehmen B unter die Grenzkosten OA herabsubventioniert werden. Das subventionsvolumen geht über die Fixkosten hinaus. Kann das Unternehmen A seine mit den Fixkosten erworbenen Renntnisse auch auf anderen Märkten verwerten, so wird es schon bei Unterschreiten der Durchschnittskosten aus dem Markt austreten. Bereits die subventionierung eines Teils der fixen Rosten reicht in diesem Fall aus, um Unternehmen $A$ zu verdrängen. Hier ist es durchaus möglich, daß die so gewonnene Innovationsrente den Subventionsbetrag übersteigt'). Für das begünstigte Unternehmen ist es immer vorteilhaft, subventionen zu verlangen, da die Innovationsrente bei ihm verbleibt.

Der diesem einfachen Modell zugrunde liegende Ralkül scheint auch in der politischen Praxis angestellt zu werden'). Es sind insbesondere Industrien mit hohen Fixkosten, auf die sich die staatliche subventionierung konzentriert (z.B. Luftund Raumfahrt, Rernenergietechnik ${ }^{3}$ )).

Doch dieser Ralkül erfaßt nur einen Teil der Problematik. Zusätzlich müssen die Anreizwirkungen solcher Interventionen für die Aktivitäten der Wirtschaftssubjekte im marktlichen und politischen Bereich beachtet werden. Die Förderung einzelner Projekte bzw. Industriezweige bedeutet ceteris paribus zugleich immer eine Diskriminierung der nicht geförderten Aktivitäten'). Die Folge dieser Verschiebung der Leistungsanreize sind eine Reduzierung der Anstrengungen im nicht geförderten Bereich zugunsten einer stärkeren Konzentration der

1) Wobei allerdings einschrănkend zu beachten ist, daß das zuerst am Markt aktive Unternehmen sich oft einen Kostenvorteil erwerben kann. Diese Tatsache erhỏht den subventionsaufwand.

2) Vgl. Klodt, H. (1987), S. $15 f$

3) Vgl. auch den Bereich der Erhaltungssubventionen: Bergbau, stahlindustrie, Schiffbau

4) Vgl. Zeppernick, R. (1985), S. $77 f$ 
Aktivitäten im den subventionierten Sektoren. Diese Umlenkung der Ressourcen in typischerweise exportorientierte Bereiche führt zum Aufbau zusätzlicher Kapazitäten mit der Folge sinkender Exportpreise. Im Zuge der marktlichen Rapitalisierung "werden also inländische Ressourcen verschenkt, so daß das Realeinkommen sinken muß."1)

Diese Entwicklung wird im politischen Bereich verstärkt. Zum einen werden die Unternehmen im betroffenen Ausland ebenfalls (Gegen-) Subventionen fordern, die zu weiteren Preissenkungen führen. Im Fall der mit staatlicher Unterstützung aufgebauten Oberkapazitäten besteht außerdem "die Gefahr, daß staatliches Engagement anschließend zu staatlicher Haftung führt,"2) mit der Folge weiterer staatlicher. Interventionen in Form von "Anpassungshilfen". Auch werden Unternehmen in anderen Bereichen, deren Konkurrenten staatlich unterstützt werden, mit dem Verweis auf die subventionierten Industrien ebenfalls staatliche Förderung verlangen ${ }^{3}$ ). Aufgrund der dargestellten politisch-ökonomischen Prozesse besteht bei Finanzhilfen, die als Obergangslösungen gedacht sind, immer die Gefahr, daß sie zu einer dauerhaften subventionierung führen. Profitieren werden in einem solchen Prozeß Volkswirtschaften mit komplementärem Güterangebot. Ihre Importe (Hochtechnologie) werden verbilligt, ihre Exporte aufgrund der Diskriminierung dieser Industrien im Ausland wettbewerbsfähiger.

Die Darstellung der Problematik des subventionswettbewerbs zeigt, daß zwar in Einzelfälen ein Ausgleich der Verzerrungen u.U. ökonomisch sinnvoll sein kann, daß aber im dadurch ausgelösten Proze $\beta$ die möglichen Vorteile des Eingriffs kompensiert werden und gesamtwirtschaftliche verluste entstehen können. Ein Verzicht auf die Teilnahme am subventionswettbewerb würde diese Verluste ausschließen. Zudem besäßen dann diejenigen Industriezweige Kostenvorteile, deren ausländische Ronkurrenz nicht gefördert wird, da sie die Finanzierung der subventionierung nicht mittragen mūssen. Auch ist zu bedenken, daß durch eine subventionierung die relativen Preise der

1) Hiemenz, U./Weiss, F.D. (1984), S. 7

2) Zeppernick, R. (1985), S. 77

3) Vgl. Zeppernick, R. (1987), S. 64 
bezuschußten Güter gesenkt werden und daß deshalb der Bezug dieser Gūter im Ausland das inländische Realeinkommen erhöht, solange die relativen Preise nicht im marktlichen Rapitalisierungsprozeß wieder ausgeglichen worden sind').

III. INNOVATIONSPOLITIR IN DER BUNDESREPUBLIK DEUTSCHLAND - EINE RRITISCHE BESTANDSAUFNAHME

1. Die Ausgaben für Forschung und Entwicklung

Die Struktur der Ausgaben des Bundes zur Förderung von Forschung und Entwicklung in der gewerblichen Wirtschaft ist durch eine Dominanz der direkten Förderung gekennzeichnet. Die Ausgaben für einzelne, bestimmte (zivile) Projekte betragen in den Jahren von 1974-1984 das 2,5 bis 15fache der Aufwendungen für indirekte Maßnahmen, mit denen allgemein Forschungs- und Entwicklungsaktivitäten gefördert werden²).

wie die Diskussion des vorangehenden Abschnitts zeigt, läßt sich eine solche Politik ökonomisch kaum begründen ${ }^{3}$, , zumal der Anteil der Grundlagenforschung an diesen Projekten gering ist4). Daß eine Technologie die Schlüsselrolle für zukünftige Entwicklungen spielen kann, läßt ein hohes Gewinnpotential bei ihrer Entwicklung vermuten und spricht dafür, daß private Wirtschaftssubjekte genügend Anreize haben werden, um in ihre Entwicklung $z$ u investieren'). Staatliche Aktivität ist hier nicht erforderlich, zumal die am Markt aktiven Wirtschaftssubjekte einen besseren oberblick über das Marktpotential einzelner Projekte haben dürften als die öffentlichen Entscheidungsträger. Bei der direkten Projektförderung ist zudem zu beachten, daß für die politischen Entscheidungsträger nur sehr geringe Anreize bestehen, Projekte, die keinen wirtschaftlichen Erfolg versprechen, abzubrechen. Während die privaten Projektträger schon aufgrund ihres Eigeninteresses

1) Vgl. Hiemenz, U./Weiss, F.D. (1984), S. $7 f$ und S. $17 f f$

2) Vgl. Bundesregierung (1986), S. 51

3) Vgl. Reck, O. (1976), S. $132 \mathrm{ff}$

4) Vgl. Bundesregierung (1986), Tab. I/11 i.V.m. Tab. I/17

5) Vgl. Keck, O. (1976), S. 132 
auf jeder stufe der Projektentwicklung erwartete Rosten und Erträge vergleichen und dabei auch konkurrierende projekte in ihren Kalkül mit einbeziehen, fällt dieser Kontrollmechanismus bei der öffentlichen Förderung weitgehend aus. Fördernde Institutionen und geförderte Unternehmen haben kein Interesse am Abbruch eines Projekts, da dies als öffentliches Eingeständnis einer Fehlentscheidung gewertet werden würde, obwohl bei Entscheidungen unter Unsicherheit Fehlschläge unvermeidlich sind. Stattdessen werden Rentabilitätsziele verdrängt und technische Erfolgskriterien in den Vordergrund gerückt' ) . Dies geschieht insbesondere bei Projekten, die unter dem Eindruck technischer Erfolge im Ausland ausgewählt wurden. Solche Vorhaben sind in erster Linie Angelegenheiten nationalen Prestiges, die dann ohne die Beachtung wirtschaftlicher Rriterien bis zum technischen Erfolg weitergeführt werden ${ }^{2}$ ). Dieser ist mit entsprechendem Zeit- und Rostenaufwand praktisch immer zu erreichen. "Doch eine noch so perfekte Technologie ist letztlich wertlos, wenn für diese Produkte kein Markt besteht oder erschlossen wird. Auch der Hinweis, man habe technisch das beste Flugzeug, den schnellsten Computer, das sicherste Auto ist ökonomisch ohne Bedeutung, wenn diese Güter nicht verkaufbar sind."3)

Als ein weiteres ziel der Forschungs- und Technologiepolitik wird ihr Beitrag zur Umwelt- und Ressourcenschonung angeführt'). Durch eine selektive Förderung von überdurchschnittlich umwelt- und ressourcenschonenden Technologien sollen die externen Rosten der Umweltverschmutzung und Ressourcenausbeutung vermieden und "gesamtwirtschaftlich unerwünschte Technologien" diskriminiert werden').

Gegen diese Begründung für eine selektive Innovationspolitik sind mehrere Einwendungen vorzubringen'). Ob eine Technologie

1) Vgl. Bundesminister der Finanzen (1985), S. $47 \mathrm{f}$

2) Vgl. Klodt, H. (1987), S. 81 ff und die Beispiele zum Flugzeugbau (S. 72ff) und zur Kernenergie (S. 77ff)

3) Zeppernick, R. (1985), S. 75

4) Vgl. Bundesminister für Forschung und Technologie (1984), S. 15

5) Vgl. Littmann, K. (1975), S. $69 \mathrm{ff}$

6) Vgl. Gutberlet, K.-L. (1984), S. $41 f$ 
"sozial unerwünsctı" ist, kann nur beurteilt werden, wenn ihren sozialen Kosten auch ihie gesamten Ertrāge gegenübergestellt werden. Dieses Informationsproblem ist jedoch bei Innovationen regelmäßg nicht gelōst, da diese mit erheblichen Unsicherheiten belastet sind.

Zudem ist eine selektive Politik in dieser Ausrichtung ineffizient, da sie die Fehlallokationen bei der Anwendung bekannter Techniken nicht $z u$ verhindern vermag.

Eine Fixierung auf die (vermuteten) sozialen Rosten neuer Technologien birgt die Gefahr, daß Projekte unterdrūckt werden, deren Nettoertrag bedeutend größer ist als der von Technologien, die zwar geringere externe Kosten verursachen, aber wirtschaftlich nicht rentabel sind.

Die hier aufgeführten sowie die bereits diskutierten Kritikpunkte sprechen gegen einen Einsatz der Forschungs- und Technologiepolitik fūr ziele der Umwelt- und Ressourcenpolitik. Stattdessen sind Maßnahmen der Internalisierung externer Effekte vorzuziehen. Durch die Gestaltung eines knappheitsgerechten Preissystems werden Unternehmen bei der Auswahl ihrer Forschungs- und Entwicklungsprojekte und beim Einsatz der bekannten Produktionsverfahren dazu veranlaßt, sich am gesamtwirtschaftlichen Gewinn $z$ orientieren. Die Internalisierungsmaßnahmen sollten dabei so angelegt sein, daß sie nicht die Anwendung bestimmter Technologien vorschreiben, sondern den Anreiz zur Entwicklung überlegener Technologien erhalten.

Die Struktur der Ausgaben für Forschung und Entwicklung blieb nicht ohne Wirkung auf die struktur der geförderten Unternehmen. Obwohl die Fördermittel für kleine und mittlere Unternehmen von 1981-1986 um über 40 Prozent gesteigert wurden, fließen ūber 80 Prozent der gesamten Mittel in Unternehmen mit mehr als 500 Beschäftigten'). Diese Ronzentration der Mittelvergabe auf relativ wenige größere Unternehmen liegt auch im Interesse der Bürokratie, denn "es ist schon zeitlich viel leichter, mit permanenten Ansprechpartnern in den stabsabteilungen der Großindustrie zu sprechen und große Förderprojekte zu bearbeiten, als mit Hunderten oder gar Tausenden von Einzelunternehmen $z u$ verhandeln."2)

1) Vgl. Bundesregierung (1986), Tab. I/21 i.V.m. Tab. I/29

2) Zeppernick, R. (1985), S. 79 
Auch ist es für die Forschungsbürokratie wesentlich einfacher, der Rritik an einem fehlgeschlagenen Projekt mit dem Hinweis auf ein renommiertes Großunternehmen zu begegnen anstelle eines kleinen, nur Fachleuten bekannten Unternehmens'). Zudem erfordern die Vergabeverfahren spezialkenntnisse und verursachen bürokratischen Aufwand. Es ist für kleine und mittlere Unternehmen oft wenig attraktiv, sich um Fördergelder zu bemühen, da sie die erworbenen Renntnisse nicht wiederkehrend nutzen kōnnen ${ }^{2}$. Die Nachteile kleiner und mittlerer Unternehmen bei der Informationsbeschaffung und -verwertung lassen sich jedoch durch den Einsatz von Subventionsberatern ausgleichen. Sie können die Informationskosten auf verschiedene Rlienten bzw. Projekte aufteilen und bieten somit eine Marktlōsung für das öffentliche Gut 'Information über Förderbedingungen' an.

Aus der Rritik an der Dominanz von Großprojekten bei der Forschungsförderung und der damit einhergehenden Konzentration der Mittel auf Großunternehmen erhob sich in der zweiten Hälfte der siebziger Jahre die Forderung nach einer stärkeren Einbeziehung kleiner und mittlerer Unternehmen in die Förderprogramme. Dieses ziel sollte vor allem durch eine Ausdehnung der indirekten Förderung sowie der auf bestimmte Unternehmensgrößen abgestellten Programme erreicht werden ${ }^{3}$ ).

Zwei Argumente werden zur Begründung der Forderung nach einer verstärkten Einbeziehung kleiner und mittlerer Unternehmen in die Forschungsförderung vorgebracht'). Zum einen soll durch eine stärkere Berücksichtigung kleiner und mittlerer Unternehmen die bestehende Bevorzugung von Großunternehmen bei Fördermaßnahmen ausgeglichen werden. Das zweite Argument

1) Vgl. Hamm, W. (1979), S. $433 f$ und Hauff, V./Scharpf, F.H. (1975), S. 55

2) Vgl. Hamm, W. (1979), S. 434

3) Zum "Forschungs- und technologiepolitischen Gesamtkonzept der Bundesregierung für kleine und mittlere Unternehmen" vgl. Bundesminister für Forschung und Technologie/Bundesminister für Wirtschaft (1978) und zur Entwicklung der Programmvolumina vgl. Bundesminister für Forschung und Technologie (1984), Tab. II/12, II/13 und II/19. Zur Kritik an dieser Politik vgl. Schrumpf, H. $(1986 / 87)$, S. $245 \mathrm{ff}$

4) Vgl. Gutberlet, R.-L. (1984), S. $42 \mathrm{ff}$ 
nimmt Bezug auf vermutete größenbedingte Unterschiede in der Forschungs- und Innovationsfähigkeit von Unternehmen. Die Forschungsförderung soll so ausgestaltet werden, daß größenbedingte Nachteile kleiner und mittlerer Unternehmen neutralisiert werden.

Die erste Begründung enthält die Oberlegung, daß die diskriminierenden Wirkungen bisheriger Maßnahmen durch zusätzliche Interventionen beseitigt werden sollen. Es handelt sich somit um ein Second-best Argument. Im folgenden soll kurz geprüt werden, $o b$ es sich bei der auszugleichenden störung um eine "irremovable distortion" handelt und inwieweit die Folgeinterventionen das angestrebte ziel erreichen. Grund für die Diskriminierung der kleinen und mittleren Unternehmen sind die direkten Fördermaßnahmen, insbesondere die Förderung von Großprojekten. Wie die Diskussion der Begründung solcher Aktivitāten ergeben hat, sind die vorgetragenen Argumente aus ökonomischer sicht nicht überzeugend. Das streben nach einer erstbesten Lösung ist deshalb vorzuziehen. Diese Einschätzung wird bestärkt, wenn zusätzlich die Wirkung der Ausgleichsmaßnahmen betrachtet wird. Diese erfolgen in Form indirekter Maßnahmen, die durch Beschränkungen der Antragsberechtigung oder des maximalen Förderbetrags kleine und mittlere Unternehmen bevorzugen sollen. Der Umfang der jeweiligen Förderung bemißt sich nach dem Ausmaß der vom Unternehmen aufzuwendenden Forschungs- und Entwicklungsausgaben. Da Forschungs- und Entwicklungstātigkeiten aber in kleinen und mittleren Unternehmen häufig nicht in eigenen Abteilungen institutionalisiert sind, sondern parallel zur Produktionsplanung, -vorbereitung und zur laufenden Produktion beim Experimentieren und "Tüfteln" unternommen werden, entsteht für die Unternehmen ein Anreiz, möglichst viele Aktivitäten mit der Assistenz "cleverer Subventionsberater" als Forschung und Entwicklung zu deklarieren'). Der seit Einführung dieser Programme beobachtete starke Anstieg der Forschungs- und Entwicklungsausgaben kleiner und mittlerer Unternehmen ist somit nicht zuletzt auf "formale" Verhaltensänderungen zurückzuführen. Ob die Maßnahmen eine effektive Ausweitung der Forschungs- und Entwicklungsaktivitäten veranlassen oder nur eine Verbesserung

1) Vgl. Wirtschaftswoche (1987a), S. 16 
der Rendite ohnehin geplanter Vorhaben über Mitnahmeeffekte bewirken, bleibt fraglich').

Aus dieser sicht sprechen sowohl der Charakter der zugrunde liegenden störung als auch die Möglichkeiten korrigierender Eingriffe gegen die Realisierung von Second-best Lösungen und für einen Verzicht auf Fördermaßnahmen.

Das zweite Argument stellt auf Unteilbarkeiten, Skalenertrăge und Diversifikationsmöglichkeiten bei Forschungs- und Entwicklungsaktivitäten sowie auf die Finanzierung dieser Tätigkeiten $a b^{2}$ ). Sind die Unterschiede in den Forschungs- und Entwicklungsanstrengungen zwischen Großunternehmen sowie kleinen und mittleren Unternehmen durch Unteilbarkeiten, Skalenerträge und unterschiedliche Diversifikationsmöglichkeiten bestimmt, so wäre es ineffizient, diese Unterschiede durch staatliche Interventionen zu beseitigen, weil dadurch Mittel in eine Verwendung gelenkt werden, in der sie geringere Grenzerträge als in nicht geförderten Alternativen erbringen. D.h. sind die Unterschiede in den Forschungs- und Entwicklungsausgaben durch größenbedingte Differenzen in den Grenzerträgen bedingt, so ist es ineffizient, diese Aktivitātsunterschiede durch statiche Fördermaßnahmen auszugleichen. Es ist auch in diesem Bereich Aufgabe des Wettbewerbsprozesses, die optimale struktur - hier: Größenstruktur - zu entdecken. Wollen auch kleinere Unternehmen vermutete Größenvorteile ${ }^{3}$ ) nutzen, so bleibt ihnen der Weg für Unternehmenskooperationen bis hin zu Unternehmenszusammenschlüssen offen'). Daß trotz dieser vermuteten Nachteile auf praktisch allen Märkten neben Großunternehmen auch kleine und mittlere Unternehmen aktiv sind, läßt den schluß zu, daß "im besonderen Maße bei diesen Unternehmen ... Flexibilitāt und Innovationskraft"o, vorhan-

1) Vgl. Tenhof, D. (1984), S. 117; Lüder, K. (1984), S. $533 \mathrm{ff}$ sowie Lichtenberg, F.R. (1984), S. $73 \mathrm{ff}$ und derselbe (1987), S. $98 \mathrm{ff}$

2) Vgl. Gutberlet, R.-L. (1984), S. $45 f f$

3) Vgl. dazu kritisch Van Cayseele, P. (1987), S. $273 \mathrm{ff}$

4) Economies of Scope müssen nicht zwangsläufig zu größeren Unternehmen führen. Sie können auch durch spezialisierung im Marktprozess genutzt werden; vgl. Teece, D.J. (1980), S. $226 \mathrm{ff}$.

5) Ministerium für Wissenschaft und Kunst Baden-Württemberg (1987), S. 28 
den sind. Nicht zuletzt auf den "Besatz mit mittelständischen Unternehmen, die flexibler seien als Großfirmen", 1) wird beispielsweise das regelmäßig über dem Bundesdurchschnitt liegende Abschneiden Baden-Württembergs in bezug auf Wirtschaftswachstum und Beschäftigungsentwicklung zurückgefūhrt.

Als weiteres Argument für spezifische Maßnahmen zugunsten kleiner und mittlerer Unternehmen wird angeführt, daß sie in der Regel keinen direkten zugang zum Kapitalmarkt haben'). Tatsächlich ist der zugang zum Rapitalmarkt an eine bestimmte Rechtsform und an weitere zulassungsvoraussetzungen gebunden, die insbesondere von kleineren und jungen Unternehmen schwer $z u$ erfüllen sind. Aber auch dieses Innovationshemmnis ist keine "irremovable distortion". Der direkte Weg zur Beseitigung dieser Mängel besteht in der Erleichterung des zugangs zum Rapitalmarkt für diese Unternehmensgruppe.

Eine Durchsicht der Förderprogramme für kleine und mittlere Unternehmen ${ }^{3}$ ) zeigt, daß für praktisch alle Bereiche der Innovationstätigkeit staatliche Programme aufgelegt wurden, daß aber der überwiegende Teil der Programme inhaltlich gerichtet ist. Dies führt insbesondere in Auswahlverfahren, die aufgrund von Budgetbeschränkungen erforderlich sind, zu einer Ronzentration der Mittel auf vermutete Schlüsselbereiche. Im Zusammenspiel zwischen dem politischen Prozeß und der Eigendynamik der Forschungsbürokratie führen ausbleibende Erfolge der Maßnahmen zu immer neuen und die unternehmerischen Entscheidungen immer stärker lenkenden Programmen'). Wurden zunächst nur Produktinnovationen gefördert, so kamen später Fertigungs- und Verfahrenstechniken hinzu. Diese wurden ergänzt durch Marketing- und Managementberatung. Schließlich

1) Euba, N. (1985), S. 306

2) Sind die Unternehmen gezwungen die Innovationsprojekte intern zu finanzieren, d.h. durch Selbstfinanzierung, sind Großunternehmen im Innovationswettbewerb eindeutig im Vorteil; vgl. Dasgupta, P./Stiglitz, J.E. (1980), S. $287 f$.

3) Vgl. auf Bundesebene Bundesminister für Forschung und Technologie (1986) und für Baden-Württemberg Ministerium für Wirtschaft, Mittelstand und Technologie Baden-Württemberg (1987) sowie den Oberblick bei Dittes, E. (1987)

4) Vgl. Staudt, E. $(1987 / 88)$, S. $102 \mathrm{ff}$ 
erfordert die Vielzahl der Programme Beratungsinstitutionen über die vorhandenen Fördermöglichkeiten ${ }^{1}$ ). Das Zusammenspiel zwischen dem Angebotsverhalten der politischen Unternehmer (vgl. Rapitel G) und dem durch "die unüberschaubar gewordene Kompliziertheit der staatlichen Maßnahmen" entstandenen "künstlichen Beratungsbedarf" sowie der unter diesen Bedingungen erzeugten subventionsmentalität führten zur Gründung von Technologieparks ${ }^{2}$, als weiterem Instrument des subventionswettbewerbs mit zweifelhaftem Erfolg ${ }^{3}$ ).

Aus ordnungspolitischer sicht stellen die mittelstandsbezogenen Fördermaßnahmen schwerwiegende Eingriffe in den marktwirtschaftichen Wettbewerbsprozeß dar. Die staatichen Interventionen lassen sich mit Marktversagen nicht begründen. Auch Second-best Argumente können insbesondere unter Einbeziehung der Informationsprobleme und der Eigendynamik politisch-ökonomischer Prozesse nicht überzeugen. Sowohl die Diskriminierung kleiner und mittlerer Unternehmen beim zugang zu Mitteln der direkten Projektförderung als auch zum Kapitalmarkt sind keine "unausrottbaren Allokationsverzerrungen"4), sondern durch staatliche Aktivitäten bzw. staatlich gesetzte Institutionen künstlich erzeugte störungen. Ein Abbau dieser störungen und die Realisierung von erstbesten Lösungen ist deshalb die optimale wirtschaftspolitische strategie.

Die zur Finanzierung der steuerreform 1990 erfolgende Beendigung der meisten indirekten Fördermaßnahmen') bedeutet einen ersten Schritt in Richtung auf ein solches optimum. Allerdings müssen die analogen direkten Finanzhilfen auch entsprechend abgebaut werden, um nicht wieder die kleinen und mittleren Unternehmen zu diskriminieren ${ }^{6}$ ).

1) Vgl. Ministerium für Wissenschaft und Runst Baden-Württemberg (1987), S. $139 \mathrm{f}$

2) Clapham, R./Scholz-Bappert, D.E. (1986), S. 62

3) Vgl. Wirtschaftswoche (1987b), S. $48 \mathrm{ff}$

4) Sohmen, E. (1976), S. 434

5) Vgl. Stoltenberg, G. (1987), S. 723 und Wirtschaftswoche (1987a), S. $17 f$

6) Vgl. dazu auch den Vorschlag des Deutschen Industrie- und Handelstages, in: Heck, H. (1987), S. 11 
Im Zentrum der steuerpolitischen Diskussion in der Bundesrepublik Deutschland steht die Gestaltung des Einkommensteuertarifs. Zum 1.1.1990 soll ein Tarif "mit völlig neuem Profil"1) in Kraft treten. Neben einer senkung des Eingangs- und des spitzensteuersatzes ist für diesen Tarif eine lineare Progressionszone vorgesehen. Zur Beurteilung der Reformwirkungen auf die Bereitschaft zur Risikoübernahme soll der geplante Tarif für 1990 dem von 1986 gegenübergestellt werden'). Dazu wird das in Abschnitt E.III.4 vorgestellte Instrumentarium verwendet. Bei steigender oder konstanter relativer Risikoaversion, also $d[\alpha(Y, U)] / d Y>0$ bzw. $d[\alpha(Y$, U) $] / d Y=0^{3}$ ), ist nur für das Einkommensintervall (59.620; 92.500) eine eindeutige Aussage möglich. während im gesamten übrigen Bereich die Partialeffekte einander entgegenwirken und der Gesamteffekt unbestimmt bleibt, erfolgt in diesem Einkommensabschnitt eine Risikodämpfung. Umgekehrt würde für $\mathrm{zu}$ versteuernde Einkommen von über 120.000 DM bei fallender relativer Risikoabneigung eine Erhöhung der Risikobereitschaft resultieren. Bei Risikoneutralität, $\alpha(Y, U)=0$, ist die Risikowirkung nur vom Jensen-Effekt abhängig, d.h. der Einkommenselastizität des marginalen Nettoeinkommens. Durch den früheren Beginn der Zone direkter Progression im Tarif von 1990 ergibt sich im Vergleich zum Tarif von 1986 im Einkommensintervall $(8.100 ; 18.035)$ eine risikodämpfende und durch die frūhere Beendigung im Interval1 (120.000; 130.031) eine risikofördernde Wirkung. Dazwischen liegt im Einkommensbereich (18.036; 59.614) eine risikofördernde und von (59.615; 120.000) eine risikodämpfende Zone.

Werden nun die Ergebnisse der empirischen Forschung zum Verlauf der Risikonutzenfunktion unter der Annahme, daß die Höhe von Vermögen und Einkommen positiv korreliert sind, auf die geplante steuerreform übertragen, so kōnnen folgende Aussagen über die Risikowirkung der Tarifänderung gemacht werden. Im

1) Kohl, H. (1987), S. 209

2) Für einen Vergleich der Tarife von 1981, 1986 und 1988 siehe Bamberg, G./Richter, W.F. (1985), S. $17 \mathrm{ff}$ und Richter, W.F. (1985), S. $1070 \mathrm{ff}$

3) Vgl. Arrow, J.K. (1971b), S. $90 \mathrm{ff}$ bzw. Sinn, H. W. (1987), S. 192ff 
Bereich hoher Einkommen (über 120.000 DM) wird die BereitBereitschaft zur obernahme wirtschaftlicher Risiken erhöht. Für niedrigere Einkommen kann keine bestimmte Wirkungsrichtung der Tarifreform abgeleitet werden, ohne daß der genaue Verlauf der Risikonutzenfunktion bekannt ist.

Wie diese Ausführungen zeigen, ist die Tarifreform unter dem Gesichtspunkt ihrer Wirkungen auf die Bereitschaft zur Risikoübernahme nicht eindeutig zu beurteilen. Auch lassen sich ansonsten wenig ökonomische Begründungen für einen linearprogressiven Tarifverlauf angeben. Aus politisch-ökonomischer Sicht ist diese steuerreform jedoch unter zwei Aspekten positiv zu beurteilen. Zum einen geben die Politiker durch den obergang von einem abschnittsweise definierten Polynom vierten Grades zu einem Tarif mit linear steigenden Grenzsteuersätzen Parameter aus der Hand, mit denen die Umverteilungswirkungen eines Tarifs für bestimmte Einkommensgruppen gezielt gesteuert werden kōnnen'). Zum anderen verschärft die steuersenkung die Budgetrestriktion und zwingt zum Abbau von steuervergünstigungen/Subventionen bzw. reduziert die Erfolgsaussichten im Wettbewerb um neue Vergünstigungen²).

So sollen zur Finanzierung der steuerreform auch verschiedene Ausnahmetatbestände beseitigt werden ${ }^{3}$ ), die insbesondere die unternehmerische Tätigkeit gegenüber abhängiger Beschäftigung diskriminieren. Beispiele sind die Besteuerung der bisher steuerfreien Lohnzuschläge für Sonntags-, Feiertags- und Nachtarbeit sowie die Besteuerung von zusätzlichen Leistungen des Arbeitgebers wie Essenszuschüssen und Belegschaftsrabatten').

1) Vgl. Bamberg, G./Richter, W.F. (1986), S. $8 f$

2) Vgl. Hiemenz, U./Weiss, F.D. (1984), S. $18 f$

3) Zu den betroffenen Vergünstigungen und Sonderregelungen vgl. Friebe, S. (1988), S. 8ff. Allerdings sollen aufgrund von Protesten einzelne Finanzierungsmaßnahmen wieder zurückgenommen oder reduziert werden.

4) Diese Kürzungen können allerdings zu einer Verteuerung des Einsatzfaktors Arbeit führen, wenn die streichungen durch Lohnsteigerungen wieder eingeholt werden. 
Neben der Reform des Einkommensteuertarifs ist auch eine Anderung des Thesaurierungssatzes der Rörperschaftsteuer Teil der steuerreform von 1990. Während der Eingangs- und der spitzengrenzsteuersatz der Einkommensteuer um jeweils drei Prozentpunkte gesenkt werden sollen, ist geplant, den Rörperschaftsteuersatz auf einbehaltene Gewinne um sechs Prozentpunkte zu reduzieren. Begründet wird das Abgehen von der bisher praktizierten Koppelung des Thesaurierungssatzes der Körperschaftsteuer an den maximalen Grenzsteuersatz der Einkommensteuer damit, daß den Unternehmen die Investitions- und Innovationsfinanzierung erleichtert werden sol11).

Diese Argumentation ist nur sehr bedingt gültig. Erstens ist dieser Vorteil nur bei der Finanzierungsform der selbstfinanzierung zu realisieren und setzt damit das Vorhandensein von Gewinnen voraus. Zum zweiten unterliegen nur körperschaftlich organisierte Unternehmen der "Körperschaft-"steuer. Die Entlastung des im Unternehmen einbehaltenen Gewinns kommt somit nur bestimmten Rechtsformen zugute.

Durch die Abkoppelung des Thesaurierungssatzes der Rörperschaftsteuer vom Spitzensteuersatz der Einkommensteuer werden Verzerrungen in zwei Richtungen verursacht2). Sind der Thesaurierungssatz der Rörperschaftsteuer und der spitzensatz der Einkommensteuer gleich hoch, so besteht in bezug auf diese steuern kein Anreiz zur Einbehaltung von Gewinnen. Im Gegenteil gibt es sogar eine begrenzte Präferenz für Ausschüttungen. Diese besteht zum einen, wenn die Erlöse aus Anteilsveräußerungen steuerpflichtig sind, da dann nur Einkommensteuer anfallt. Zum zweiten ist aufgrund der Progression des Einkommensteuertarifs Ausschüttung und Wiedereinlage des Gewinns solange vorteilhaft, wie die einkommensteuerliche Belastung der letzten ausgeschütteten Geldeinheit geringer als der steuersatz bei Einbehaltung der Gewinne ist ${ }^{3}$ ). Die Vorteilhaftigkeit des Schütt-aus-Hol-zurück-Verfahrens wird allerdings durch die Rirchen- und die Gesellschaftsteuer beeinträchtigt. Erfolgt die Wiedereinlage dagegen in Form von Ge-

1) Vgl. Kohl, H. (1987), S. 27

2) Zum Folgenden vgl. Wissenschaftlicher Beirat beim Bundesministerium der Finanzen (1987), S.23ff

3) Vgl. Kapitel F, Abschn. III.1.1 
sellschafterdarlehen, fällt keine Gesellschaftsteuer an und zudem kann die steuerliche Begünstigung der Fremdfinanzierung von Kapitalgesellschaften bei der Gewerbe- und Vermögensteuer genutzt werden. Allerdings ist die praktische Durchführbarkeit des Schütt-aus-Hol-zurück-Verfahrens auf kleinere, personenbezogene Rapitalgesellschaften begrenzt. Die Senkung des Rörperschaftsteuersatzes auf einbehaltene Gewinne unter den Spitzengrenzsteuersatz der Einkommensteuer bewirkt eine steuerliche Präferenz der Gewinnthesaurierung. Diese hat nicht nur die Diskriminierung der Außenfinanzierung gegenüber der Selbstfinanzierung zur Folge, sondern verhindert auch durch die Ausschaltung der Selektionsfunktion des Marktes eine Lenkung der Ressourcen in die Verwendung mit dem höchsten Grenzertrag'). Benachteiligt sind dadurch insbesondere Unternehmen, deren laufenden Aufwendungen Erlöse gegenüberstehen, die erst in späteren Perioden anfallen und die deshalb auf externe Rapitalzufuhr angewiesen sind. Typischerweise sind es innovative Unternehmen, die durch eine solche zahlungsstromstruktur beschrieben sind. Die Abkoppelung des Körperschaftsteuersatzes auf einbehaltene Gewinne vom spitzensatz der Einkommensteuer fūhrt somit zu einer Einschränkung der Funktionsfähigkeit des Marktes und zu einer Diskriminierung kapitalsuchender, innovativer Unternehmen.

Zum zweiten erfolgt eine Diskriminierung zwischen Rechtsformen. In einer Personengesellschaft unterliegt auch der im Unternehmen verbleibende Gewinn der Einkommensteuer. Bei einem Rirchensteuersatz von neun Prozent bedeutet dies, daß bereits beginnend mit einem persönlichen Einkommensteuersatz von 47,85 Prozent bzw. einem Einkommen von 103.040 DM die Einbehaltung von Gewinnen in einer Personengesellschaft stärker als bei der Thesaurierung in einer Kapitalgesellschaft belastet wird.

1) Vgl. zur Rritik an dieser geplanten Anderung Bareis, P. (1987), S. 67ff 
Mit dem ziel, die Schranken zur Beschaffung von Eigenkapital über den Kapitalmarkt für kleine und mittlere Unternehmen zu senken, wurde zum 1. Mai 1987 ein neues Börsensegment eingeführt. Dieser geregelte Markt ist unterhalb des amtlichen Marktes angesiedelt und die Anforderungen, die an die zulassung beantragende Unternehmen gestellt werden, sind gegenüber den im Rahmen der Harmonisierung der Zulassungsanforderungen in der europäischen Gemeinschaft verschärften Zulassungsbedingungen für den Börsenhandel mit amtlicher Notierung reduziert' ${ }^{\prime}$. Während die zulassungsvoraussetzungen für den amtlichen Markt durch Rechtsverordnung von der Bundesregierung bestimmt werden (\$ $38 I$ BörsG), sind "die näheren Bestimmungen für den geregelten Markt" in den jeweiligen Börsenordnungen zu treffen ( $\$ 72 I$ BörsG). Diese stimmen jedoch auf den verschiedenen Börsenplātzen weitgehend miteinander überein, da die Börsenordnungen den in der Arbeitsgemeinschaft deutscher Wertpapierbörsen getroffenen Absprachen entsprechen ${ }^{2}$ ). Die für die emissionswilligen Unternehmen wichtigsten Unterschiede zwischen dem amtlichen und dem geregelten Markt betreffen die Anforderungen an das Alter des Unternehmens ( 3 Jahre bzw. keine Begrenzung), den Mindestemissionsbetrag (2,5 Mio DM bzw. 0,5 Mio DM) sowie die Form der Publizität (Veröffentlichung in Börsenpflichtblättern bzw. Wahlmöglichkeit zwischen Börsenpflichtblättern und der Auslage bei Zahlstellen (Schalterpublizität)). Die zulassung ist auf beiden Märkten vom Emittenten zusammen mit einem Rreditinstitut zu beantragen, das an einer inländischen Wertpapierbörse zum Handel zugelassen ist. Abweichend davon kann für die zulassung zum geregelten Markt auch ein anderes Unternehmen als ein Kreditinstitut die Emission begleiten, sofern es die fachliche Eignung und

1) $\mathrm{Zu}$ den Zulassungskriterien zum amtlichen und geregelten Markt vgl. den ausführlichen Oberblick in: Kommission "Zweiter Börsenmarkt" (1987), S. $18 \mathrm{ff}$

2) Die folgenden Angaben sind der Börsenordnung der Baden-Württembergischen Wertpapierbörse zu stuttgart entnommen. 
die erforderliche zuverlässigkeit besitzt (\$ 71II BörsG, \$ 45 II BörsO)1'.

Während für den amtlichen Markt dem zulassungsantrag ein von beiden Antragstellern unterschriebener Prospekt beizufügen ist, reicht für den geregelten Markt ein Unternehmensbericht aus. Dieser hat zwar weitgehend gleichen inhaltlichen Anforderungen $z u$ genügen, muß aber nicht vom emissionsbegleitenden Unternehmen unterschrieben werden. Der Emissionbegleiter haftet somit nicht für den Inhalt des Unternehmensprospekts.

Dieser kurze Oberblick über die Anderungen im Kapitalmarktrecht zeigt, daß die Anforderungen an den Emittenten und das emissionsbegleitende Unternehmen für die zulassung zum geregelten Markt gegenüber dem amtlichen Markt niedriger und insbesondere weniger kostenträchtig sind, da neben den reduzierten Publizitātsanforderungen auch nur die halben sātze der Gebührenordnungen der Börsen gelten.

Ob die Einführung des Marktabschnitts "Geregelter Markt" tatsächlich eine offnung des Kapitalmarkts für innovative, kleine und mittlere Unternehmen bewirken kann, ist indessen fraglich. Zum einen trägt dieses neue Marktsegment nicht zu einer differenzierteren vertikalen struktur der Teilmärkte bei, da es darauf angelegt ist, die bislang im geregelten Freiverkehr gehandelten Titel aufunehmen ( $\$ 97$ I BörsG). Die bereits kritisierte unzureichende Abstufung der Börsensegmente wird nicht beseitigt2'.

Der zweite Punkt, der insbesondere eine offnung der privatrechtlich organisierten Marktabschnitte geregelter und ungeregelter Freiverkehr verhindert, ist der weitgehend fehlende Wettbewerb der Börsen als Marktorganisatoren ${ }^{3}$ ). Die Politik der Börsen wird vor allem durch die Banken bestimmt, die in

1) Man beachte aber die Einschrănkung in \$45II Satz 3 Börso, wonach der Emissionsbegleiter einen ordnungsgemåßen Börsenhandel gewăhrleisten muß. Diese Voraussetzung kőnnen praktisch nur Börsenmitglieder, also Kreditinstitute und Freimakler, erfüllen; vgl. Schwark, E. (1987), S. $2045 f$ und ebenso kritisch Kommission "Zweiter Börsenmarkt" (1987), S. $28 f$.

2) Vgl. Giersch, H./Schmidt, H. (1986), S. 78

3) Vgl. Giersch, H./Schmidt, H. (1986), S. 79 
den Börsenvorständen und Freiverkehrsausschūssen dominieren. Zudem wird das Vorgehen der Börsen in der Arbeitsgemeinschaft deutscher Wertpapierbörsen abgestimmt'). Da der Ronflikt zwischen dem Anlegerschutz und der Erleichterung des Marktzugangs in erster Linie durch Risikoausschluß und weniger durch Risikotransparenz gelöst wird2), und zudem fachliche Eignung und Zuverlässigkeit von anderen Emissionsbegleitern als Kreditinstituten von den Börsenorganen beurteilt werden, kann durch das neue Marktsegment die Kapitalmarktöffnung für innovative, risikoreiche Unternehmen kaum erreicht werden.

Durch zwei weitere, zum 1. Januar 1987 in Kraft getretene gesetzliche Maßnahmen sollen die Möglichkeiten der Eigenkapitalbeschaffung kleiner und mittlerer Unternehmen verbessert werden.

Mit dem Gesetz zur Verbesserung der Rahmenbedingungen für institutionelle Anleger ${ }^{3}$ ) werden die Beschränkungen für Versicherungen und Kapitalanlagegesellschaften, Beteiligungskapital zur Verfügung zu stellen, gelockert. Der Anlagekatalog für Versicherungen wird über im amtlichen Handel oder geregelten Freiverkehr gehandelte Aktien sowie Anteile an Wertpapiersondervermögen hinaus auch auf sonstige Aktien, Geschäfsanteile an GmbH, Kommanditanteile, Beteiligungen als stille Gesellschafter und Genußrechte erweitert. Außerdem wird die für Versicherungen und Rapitalanlagegesellschaften geltende Begrenzung des Anteilsbesitzes von fünf Prozent auf zehn Frozent des Grundkapitals aufgestockt'). Diese Erhöhung des zulässigen Anteilsbesitzes an einem Unternehmen macht ein Engagement in kleinen und mittleren Unternehmen für institutionelle Anleger oft erst lohnend, da die Transaktionskosten fix sind und deshalb ein bestimmtes Mindestbeteiligungsvolumen erforderlich ist').

1) Vgl. die abgestimmte Ausgestaltung des geregelten Markts und der Gebuhrenordnung

2) Vgl. Weichert, R. (1985), S. 18

3) BGB1. 1986 I, S. $2485 \mathrm{ff}$

4) Fũr andere Beteiligungsobjekte als Aktiengesellschaften gilt diese Begrenzung analog.

5) Vg1. Weichert, R. (1985), S. $26 \mathrm{ff}$ 
Mit dem Gesetz über Unternehmensbeteiligungsgesellschaften ${ }^{1}$ ) soll nicht börsennotierten Unternehmen eine indirekte Aufnahme von Eigenkapital am Rapitalmarkt ermöglicht werden und zugleich breiteren Anlegerschichten Zugang zu einer Beteiligung an solchen Unternehmen eröffnet werden. Diese ziele sollen erreicht werden, indem eine Unternehmensbeteiligungsgesellschaft (UBG) Minderheitsbeteiligungen an Aktiengesellschaften, Gesellschaften mit beschränkter Haftpflicht und Rommanditgesellschaften bzw. als stiller Gesellschafter erwirbt. Die UBG ist in der Rechtsform der AG zu betreiben und hat ihr Grundkapital von mindestens zwei Millionen DM zu mindestens siebzig Prozent über ein offentliches Angebot am Rapitalmarkt zu finanzieren. Durch die Befreiung der UBG von der Vermögenund Gewerbesteuer wird der Erwerb von Aktien einer UBG steuerlich gleichgestellt mit einer direkten Beteiligung an einem Unternehmen. Ob die UBG zu einem wirksamen Instrument der Finanzierung innovativer Unternehmen werden, muß bezweifelt werden') . Zwar können UBG eine Politik des Risiko-Pooling betreiben, aus Kostengründen dürfte allerdings die Zahl der einbezogenen Unternehmen relativ gering sein. Zudem stellt sich bei der Refinanzierung der UBG das Problem, daß die (potentiellen) Anleger das Portefeuille der UBG aufgrund mangelnder Transparenz und Informationsverarbeitungskapazitäten kaum bewerten können.

\section{4.} Die Wettbewerbspolitik

Unbeschränkter Marktzugang ist die Grundlage dafür, daß in einem marktlichen Wettbewerbsprozeß die optimale Innovationsintensität entwickelt wird. Wie in Rapitel D gezeigt wurde, führen parallele Aktivitäten der Wettbewerber und der von ihnen gegenseitig ausgeübte Druck zu einem Wettbewerbsklima, in dem neuartige wirtschaftliche Rombinationen gesucht und vermarktet werden.

Allerdings ist der Marktzugang in zahlreichen Bereichen beschränkt. Bereits angesprochen wurden die Probleme kleiner

1) BGBl. 1986 I, S. $2488 \mathrm{ff}$

2) Zur Kritik vgl. Schwark, E. (1987), S. 2048 
und mittlerer Unternehmen bei der Beschaffung von Eigenkapital. Außerdem werden die Möglichkeiten zu wirtschaftlicher Betätigung durch Auflagen und aufwendige bürokratische Genehmigungsverfahren eingeengt' '. Besonders kritisch zu betrachten ist in diesem Zusammenhang die unterschiedliche Behandlung neu eintretender und etablierter Unternehmen beispielsweise bei der Anwendung von Umweltschutzauflagen.

Auch industriepolitische Interventionen haben marktabschlieBende Wirkungen ${ }^{2}$ ). So werden durch Erhaltungssubventionen nicht nur relative Preise verzerrt und Neuerungen diskriminiert, sondern wird oft auch durch begleitende Regulierungsmaßnahmen der Markteintritt neuer Wettbewerber verhindert ${ }^{3}$ ). Weitere Wettbewerbsbeschränkungen ergeben sich aus der Existenz von Kartellen, Monopolen und der Anwendung von Behinderungspraktiken. So wird zum Beispiel im Bereich des Fernmeldewesens die Entwicklung nicht durch den Wettbewerb als Entdeckungsverfahren sondern durch die zulassungskriterien der Bundespost gelenkt4). Auch können beispielsweise nach dem Gesetz gegen Wettbewerbsbeschränkungen zulässige Rationalisierungskartelle neuartige organisationsformen verhindern.

IV.

FAZIT

Die struktur der innovationspolitischen Aktivitäten in der Bundesrepublik Deutschland zeigt, daß die staatlichen Maßnahmen überwiegend induzierend angelegt, $d . h$. ergebnisorientiert sind. Diese Ausrichtung der innovationspolitischen Maßnahmen steht den offiziellen Erklärungen zu den zielsetzungen der Wirtschaftspolitik entgegen. In ihnen wird auf die Gestaltung der ordnungspolitischen Grundlagen abgestellt, um die Rahmenbedingungen für die suche nach Innovationen im marktwirt-

1) Vgl. den Katalog von Innovationshemmnissen in Institut für Wirtschaftspolitik an der Universität zu Köln/Ifo-Institut für Wirtschaftsforschung München (1982), S. $378 \mathrm{ff}$

2) Vgl. die Ausführungen zum Subventionswettbewerb und zu den Kapitalisierungsprozessen

3) Vgl. hierzu die Problematik von Sanierungsfusionen und Sonderkartellen

4) Vgl. Wissenschaftlicher Beirat beim Bundesminister für Wirtschaft (1986), S. $21 \mathrm{ff}$ 
schaftlichen Wettbewerbsprozeß zu schaffen. Somit erfolgt zwar eine prinzipielle Festlegung auf eine ordnungspolitisch orientierte, akkommodierende Politik, daneben werden aber mit dem Verweis auf das Vorliegen von Marktversagen induzierende Maßnahmen begründet. Externe Effekte können nur im Bereich der Grundlagenforschung staatliche Fördermaßnahmen begründen. Die Externalitäten, die bei angewandter Forschung und Entwicklung auftreten, rechtfertigen dagegen keine staatlichen Interventionen. Die Instrumente des gewerblichen Rechtschutzes sowie das zur verwertung von Innovationen erforderliche Know-how erlauben es dem Innovator, sich die Erträge seiner Anstrengungen anzueignen. Zudem wirkt der Wettbewerbsdruck den anreizhemmenden Effekten von Externalitäten entgegen. Auch aus der Unsicherheit, die für zukunftsgerichtete und insbesondere für innovative Aktivitäten charakteristisch ist, kann keine Notwendigkeit für staatliche Eingriffe abgeleitet werden. Zum einen gehen in den marktlichen Abstimmungsproze $\beta$ auch Erwartungen über zukünftige Entwicklungen ein, da Angebots- und Nachfrageentscheidungen Teil der Wirtschaftsplanung der Marktteilnehmer sind. Zum zweiten kann nicht davon ausgegangen werden, daß politische Unternehmer die Chancen und Risiken bestimmter Projekte besser einschätzen können als private Wirtschaftssubjekte, die am Markt aktiv sind. Zudem spricht der kurze Planungshorizont der politischen Entscheidungsträger gegen staatliche Interventionen. Auch aus Unteilbarkeiten kann kein staatlicher Handlungsbedarf abgeleitet werden, da bei entsprechenden Ertragserwartungen im marktwirtschaftlichen Prozeß Rooperationsformen entwickelt werden, welche die Realisierung von Projekten erlauben, die das Finanzierungspotential von einzelnen Unternehmen übersteigen. Die Analyse des Subventionswettbewerbs zeigt, daß eine subventionierung der inländischen Innovationsaktivitäten in Einzelfällen zwar positive Wohlfahrtseffekte haben kann, daß aber solche Interventionen bei Berücksichtigung der auftretenden Kapitalisierungsprozesse im marktlichen und im politischen Bereich längerfristig ein Negativ-summen-spiel darstellen.

Die Förderung kleiner und mittlerer Unternehmen wird mit Second-best Argumenten begrūndet. Sowohl die Diskriminierung dieser Unternehmen beim Zugang zu staatlichen Fördermitteln 
als auch beim zutritt zum Kapitalmarkt sind durch statiche Interventionen erzeugte, kūnstliche störungen. Die optimale wirtschaftspolitische strategie ist deshalb der Abbau dieser störungen und die Realisierung einer erstbesten Lösung.

Ober die Wirkung der geplanten Reform des Einkommensteuertarifs auf die Bereitschaft zur Risikoübernahme können nur im Bereich hoher Einkommen eindeutige Aussagen gemacht werden. Hier wird die Risikobereitschaft gefördert. Liegen die Einkommen unter 120.000 DM, dann wirken die Partialeffekte einander entgegen und der genaue Verlauf der Risikonutzenfunktion muß bekannt sein, um die Risikowirkungen der Tarifreform bestimmen zu können. Die Abkoppelung des Thesaurierungsatzes der Rörperschaftsteuer vom spitzensatz der Einkommensteuer ruft zusätzliche Verzerrungen zwischen den Finanzierungsformen und den Rechtsformen der Unternehmen hervor. Die Senkung des Thesaurierungssatzes der Rörperschaftsteuer wird deshalb negativ beurteilt.

$\mathrm{Ob}$ die Einfūhrung eines zusătzlichen Börsensegments die Möglichkeiten der Eigenkapitalbeschaffung für neugegründete sowie für kleine und mittlere Unternehmen verbessert, ist aufgrund der struktur der Zulassungsverfahren zum Börsenhandel fraglich.

Zahlreiche Regulierungsmaßnahmen hemmen wettbewerbliche Prozesse und erschweren den Marktzutritt neuer Unternehmen bzw. blockieren die Einführung von Innovationen. Zudem können sich Unternehmen in Ausnahmebereichen durch Maßnahmen im Rahmen der selbstregulierung vor neuer Konkurrenz schützen.

$\underline{\mathbf{V}}$ VORSCHLAGE FOR MASSNAHMEN IM RAHMEN EINER AKROMMODIERENDEN INNOVATIONSPOLITIK

Im folgenden werden der in der Bundesrepublik Deutschland gegenwārtig praktizierten Politik, deren konkrete Maßnahmen induzierend angelegt sind und deren Regelungsintensität im politischen Prozeß ständig erhöht wird, Vorschläge für eine akkommodierende Politik gegenübergestellt. Sie sind auf die Beseitigung von Diskriminierungen und Hemmnissen fūr innovative Aktivitäten ausgerichtet. Die folgenden Ausführungen beruhen auf den Analysen der einzelnen Kapitel dieser Arbeit und sind 
in drei Bereiche gegliedert. Erstens werden für das Gebiet der steuerpolitik Vorschläge diskutiert, welche die Diskriminierung zwischen risikolosen und risikobehafteten Aktivitäten, zwischen alternativen Finanzierungsformen und zwischen verschiedenen Rechtsformen der Unternehmenstätigkeit zum ziel haben. Zweitens werden für den Bereich der Rapitalmarktpolitik Anderungen vorgeschlagen, die eine offnung der Märkte zur Finanzierung risikobehafteter Projekte und eine Abstufung der Börsensegmente entsprechend dem Anlagerisiko berwirken sollen. Drittens wird für einen Verzicht auf lenkende, induzierend angelegte staatliche Programme plädiert. stattdessen wird eine Beschränkung industriepolitischer Aktivitäten auf Maßnahmen vorgeschlagen, die eine Verbesserung der Infrastruktur anstreben und die zu einer verstärkten Förderung der Grundlagenforschung führen.

1. Maßnahmen im Bereich der Steuerpolitik

In diesem Abschnitt werden drei Bereiche steuerlicher Diskriminierung diskutiert: Erstens die Diskriminierung risikobehafteter Aktivitäten durch die gegenwärtigen Regelungen des Verlustausgleichs; zweitens die steuerliche Ungleichbehandlung alternativer Finanzierungsformen durch die Gewerbe- und Vermögensteuer und drittens die vermögensteuerliche Doppelbelastung des Anteilsbesitzes an Rapitalgesellschaften.

Wie in den Kapiteln $E$ und $H$ gezeigt wurde, ist es nicht möglich, die Risikobereitschaft über Anderungen des Tarifverlaufs zielgerichtet $z$ beeinflussen. Allerdings wurden die gesetzlichen Regelungen zur Verlustkompensation als steuerliche Institutionen identifiziert, die einseitig risikobehaftete Aktivitäten betreffen und die deshalb zur Einflußnahme auf die Risikobereitschaft eingesetzt werden können. Nach den derzeit geltenden Regelungen kann ein sofortiger Verlustausgleich nur dann erreicht werden, wenn das zu versteuernde Einkommen (bzw. auf Körperschaftsebene: das Einkommen abzüglich der Ausschüttungen) in den beiden Jahren, die dem Verlustjahr vorangehen, positiv ist. Da diese Bedingung von körperschaftich organisierten jungen Unternehmen aufgrund von 
Anlaufverlusten in der Regel nicht erfüllt werden kann, wird eine Gesetzesänderung zur Neuregelung des Verlustausgleichs notwendig, wenn die Liquiditäts- und Finanzierungsbedingungen insbesondere junger Unternehmen verbessert werden sollen und damit bessere Voraussetzungen für innovative Neugründungen geschaffen werden sollen. Der Vorschlag umfaßt im einzelnen folgende Komponenten:

1. Die Beschränkungen der Verlustkompensation im Rahmen des einkommensteuerlichen Verlustausgleichs nach \$ 10d EStG werden aufgehoben. Der steuerpflichtige hat ein nicht reversibles Wahlrecht über die Aufteilung seines Verlusts auf Verlustrücktrag und Verlustvortrag. Der Verlustrücktrag bzw. Verlustvortrag erfolgt rückschreitend bzw. fortschreitend vom Verlustjahr an, bis der Verlust ausgeglichen ist; d.h. Jahre mit geringerem Einkommen und deshalb niedrigeren steuersätzen können nicht übersprungen werden.

2. Auf der Ebene der Kapitalgesellschaft besteht ein Wahlrecht zwischen Verlustvortrag und der Ausgabe von steuergutscheinen. Diese dürfen ausgefertigt werden, wenn der verlust festgestellt ist. Sie sind auf andere Kapitalgesellschaften oder auf die Gesellschafter übertragbar. ober die Verwendung des Verlusts entscheidet die Gesellschafter-bzw. die Hauptversammlung.

3. Der gewerbesteuerliche Verlustausgleich wird auf einen unbegrenzten Verlustvortrag ausgedehnt.

Dieser Vorschlag zur Reform des steuerlichen Verlustausgleichs ist an einem ähnlichen Vorstoß des Sachverständigenrates zur Begutachtung der gesamtwirtschaftlichen Entwicklung orientiert''. Die Beschränkung der Obertragbarkeit von steuergutscheinen auf die Gesellschafter der betreffenden Rörperschaft und auf Rapitalgesellschaften verhindert auf der Ebene der Erwerber "ein Abweichen vom Grundsatz der Besteuerung nach der Leistungsfähigkeit."2) D.h. es wird ausgeschlossen, daß steuerpflichtige mit hohen $z u$ versteuernden Einkommen ihre persönliche steuerbelastung dadurch reduzieren, daß sie

1) Vgl. Sachverständigenrat (1984), S. 216

2) Arbeitsgruppe Steuern (1987), S. 16 
von Rapitalgesellschaften mit negativen Einkommen Verlustgutscheine kaufen. Allerdings verbleibt beim Handel zwischen Rörperschaften ein Finanzierungsvorteil, wenn die Differenz zwischen dem Thesaurierungssatz der körperschaftsteuer und den Grenzsteuersätzen der Anteilseigner die Transaktionskosten übersteigt.

Neben der Reform des Verlustausgleichs erscheint im Bereich der steuerpolitik die Beseitigung der Diskriminierung einzelner Finanzierungs- bzw. Rechtsformen vorrangig.

Schon vor einer umfassenden Neuordnung des Gemeindesteuersystems sollte im sinne einer inkrementalen Reform die gewerbesteuerliche Diskriminierung der Eigenfinanzierung beseitigt werden. Die Aufkommenserhöhung aus der vollen Hinzurechnung der Fremdkapitalzinsen bzw. des Fremdkapitals als Dauerschuldzinsen bzw. Dauerschulden zu den Bemessungsgrundlagen der Gewerbesteuer könnte zur Abschaffung der ohnehin wenig ergiebigen und kostenaufwendigen Gesellschaftsteuer verwandt werden.

Steuersystematisch wäre eine Beseitigung der vermögensteuerlichen Doppelbelastung des Anteilsbesitzes an Rapitalgesellschaften auf der Ebene der Rapitalgesellschaften vorzuziehen. Dadurch würden jedoch nicht die Verzerrungen beseitigt, die dadurch entstehen, daß das Betriebsvermögen bei Personengesellschaften und Einzelunternehmen mit dem Teilwert bewertet wird, während der Anteilsbesitz an Kapitalgesellschaften mit dem Kurswert oder dem gemeinen Wert angesetzt wird. Deshalb sollte die vermögensteuerliche Doppelbelastung auf der Ebene der Anteilseigner beseitigt werden. Das Betriebsvermögen ist für Zwecke der Vermögensteuer dann bei allen Rechtsformen mit dem Teilwert anzusetzen.

Um zusätzliche Verzerrungen zwischen Rechtsformen und Finanzierungswegen zu verhindern, sollte auf die geplante "Spreizung" des Thesaurierungssatzes der Rörperschaftsteuer und des spitzensatzes der Einkommensteuer verzichtet werden ${ }^{1}$ ).

Eine steuer, die Rapitalmarkttransaktionen verteuert und den Börsenplatz Bundesrepublik Deutschland im internationalen

1) Vgl. Bareis, P. (1987), S. 70 
Wettbewerb belastet, ist die Börsenumsatzsteuer. Ihre Abschaffung würde den Handel mit Wertpapieren verbilligen und die Attraktivität der inländischen Börsen gegenüber dem Ausland erhöhen.

2. Maßnahmen im Bereich der Kapitalmarktpolitik

Um die Kapitalmärkte auch für junge, kleine und mittlere Unternehmen zu öffnen, erscheinen folgende Maßnahmen geeignet: ${ }^{1}$ )

1. Neben den (Universal-)Banken werden spezielle Investmentbanken, die nur das Emissions- und das Depotgeschäft betreiben, zum Effektenmarkt zugelassen. Diese Investmentbanken unterliegen einer speziellen Effektenmarktaufsicht.

2. Das Antragsrecht auf Zulassung bzw. Einbeziehung von Unternehmensanteilen zum Börsenhandel steht neben den Rreditinstituten auch den Investmentbanken $z u$.

3. Die Zulassungskriterien sind entsprechend den angestrebten Börsensegmenten abzustufen. Insbesondere für die Einbeziehung nicht aktienrechtlich organisierter Unternehmen in den geregelten oder ungeregelten Freiverkehr legen die einzelnen Börsen die Anforderungen in den Börsenordnungen eigenständig fest ${ }^{2}$.

Mit den vorgeschlagenen Maßnahmen soll erreicht werden, daß sich ein wirksamer Emissionswettbewerb entwickelt und daß auch kleine und stark risikobehaftete Unternehmen, die bisher keine Börsenbegleiter finden konnten, da diese um ihr standing fürchteten, ein Emissionshaus finden. Dieser offnung des Emissionsgeschäfts entspricht auf dem Sekundärmarkt ein Antragsrecht der Investmentbanken. Mit der Abstufung der Börsensegmente soll Anlegerschutz durch Risikotransparenz erreicht werden. Bei einer entsprechenden segmentierung ist

1) Vgl. Giersch, H./Schmidt, H. (1986), S. $65 f f$

2) In verschiedenen europäischen Ländern und den USA liegen vielfältige Erfahrungen mit abgestuften Börsensegmenten vor; vgl. Claussen, C.P. (1984), S. 5ff; Schmidt, H. (1987), S. 289ff und Reul, J. (1986), S. $70 \mathrm{f}$. 
auch der Handel von Anteilen an Nicht-Aktiengesellschaften denkbar'). Die Voraussetzungen sind von den einzelnen Börsen festzulegen. Allerdings dürfte der Handel in solchen Anteilen aufgrund der geringen standardisierung im Vergleich zu Aktientransaktionen mit hohen Rosten verbunden sein. Dem Gestaltungsbedürfnis kleiner und mittlerer Unternehmen und dem der Alt-Inhaber kōnnte beispielsweise auch dadurch Rechnung getragen werden, daß als Romplementär einer Kommanditgesel1schaft auf Aktien eine GmbH zugelassen würde.

\section{Maßnahmen im Bereich der Industriepolitik}

Wie die Analyse in den Kapiteln $\mathrm{G}$ und $\mathrm{H}$ dieser Arbeit ergibt, sind induzierende innovationspolitische Maßnahmen ökonomisch nicht begründbar. Deshalb sind die Förderaktivitäten der verschiedenen Gebietskörperschaften unter diesem Aspekt zu überprüfen und gegebenenfalls zu streichen. Dies betrifft insbesondere die Förderung einzelner Techniken sowie der angewandten Forschung und Entwicklung und die subventionierung bestimmter Unternehmensgruppen und Branchen ${ }^{2}$ ).

Sind zur Erfüllung öffentlicher Aufgaben Forschungs- und Entwicklungsarbeiten erforderlich, so können diese entweder von der zuständigen Dienststelle selbst durchgeführt werden oder es können von ihr entsprechend spezifizierte Forschungsaufträge vergeben werden. Die Vergabe der Mittel und die Kontrolle der Arbeiten obliegt somit der fachlich zuständigen Behörde ${ }^{3}$. Die Zuständigkeit des Bundesministeriums für Forschung und Technologie beschränkt sich dann auf die Vergabe von Mitteln für die Grundlagenforschung.

1) Vgl. dazu ausführlich Kommission "Zweiter Börsenmarkt" (1987), S. $86 f f$

2) Vgl. dazu Molitor, B. (1985), S. $300 f$

3) Vgl. Keck, O. (1976), S. 132f; in dieser Weise sind Forschung und Entwicklung in Verteidigungsbereich bereits organisiert. 
Die auf den Abbau von Diskriminierungen bzw. Privilegien und die offnung von Rapital- und Gütermärkten zielende Politik gefährdet Besitzstände und ruft deshalb im politischen Proze $\beta$ Widerstände hervor. Die vorgeschlagenen Maßnahmen stellen Anderungen innerhalb des gegebenen politisch-konstitutionellen Systems dar. Da bei wirtschaftspolitischen Entscheidungsträgern eine verbreitete obereinstimmung über die Bedeutung der Innovationspolitik besteht und da zudem die Budgetrestriktion der offentlichen Haushalte durch die gegenwärtige wirtschaftliche Entwicklung verstärkt wirksam wird, dürfen die Voraussetzungen zur Implementation der Vorschläge für eine akkommodierende Innovationspolitik als gut bezeichnet werden'1). Anders sind dagegen die Chancen für eine Implementation grundlegender, konstitutioneller Regelungen zu bewerten. Sie ändern die Rahmenbedingungen, innerhalb derer die politischökonomischen Prozesse ablaufen und führen dazu, daß die Handlungsparameter der politischen Unternehmer begrenzt und die Erfolgsaussichten von Umverteilungsaktivitäten bzw. die Unbeständigkeit der Gesetzgebung reduziert werden ${ }^{2}$ ). Das Problem der Implementation konstitutioneller Regelungen, welche die Handlungsmöglichkeiten der politischen Unternehmer grundlegend beschränken, muß als bislang nicht gelöst gelten.

1) Zu der Abhăngigkeit der Implementationschancen von Reformprogrammen von der politisch-őkonomischen situation vgl. Folkers, C. (1986), S. $31 \mathrm{f}$.

2) Zu einem solchen Vorschlag vgl. Mann, H. (1987), S. $214 f$ 
I. ZUSAMMENFASSUNG

Auf die Verlangsamung und Stagnation der Entwicklung des Sozialprodukts wird in der wirtschaftspolitischen Diskussion mit der Forderung nach einer "Neuen Industrie- und Forschungspolitik" reagiert. Mit einer solchen Politik sollen zwei zielsetzungen verfolgt werden. Zum einen soll der Verlust von Arbeitsplätzen in traditionellen Industriezweigen an Schwellenländer durch eine Revitalisierung dieser Branchen gestoppt werden. Zum anderen sollen durch die Förderung zukunftsträchtiger Industrien neue Märkte erschlossen werden. Insbesondere hinter der letztgenannten zielvorstellung steht die Vermutung, daß es die Innovationstätigkeit ist, die das Wirtschaftswachstum bestimmt. Um diese Hypothese zu überprüfen, wurden zunächst die Determinanten des Wirtschaftswachstums aufgegliedert und diskutiert.

Empirische Untersuchungen zeigen zwei bedeutsame Tendenzen in der Produktivitätsentwicklung. Zum einen ist das Potential der Determinanten, die in der Vergangenheit das Wachstum der Produktivität entscheidend getragen haben, weitgehend ausgeschöpft. Dies betrifft insbesondere die Möglichkeit der Produktivitätssteigerung durch Reallokation des Faktors Arbeit. Aber auch das Potential, durch bessere Ausbildung die Qualität des Faktors Arbeit zu verbessern, wird zunehmend ausgeschöpft. Diese abnehmenden steigerungsmöglichkeiten werden gleichzeitig durch ständig wachsende negative Einflüsse aus dem Umfeld der Unternehmen verstärkt. Sie bestehen in der wachsenden Regulierungsintensität durch staatliche Auflagen im Produktionsbereich sowie in der obertragung unentgelticher Dienstleistungspfichten auf die privaten Wirtschaftssubjekte.

Nachdem die Innovationstätigkeit zunächst in ihren gesamtwirtschaftlichen Zusammenhang gestellt worden war, wurden anschließend die einzelwirtschaftlichen Grundlagen von Innovationsentscheidungen analysiert. Ausgangspunkt ist in Rapitel $c$ ein einfaches neoklassisches Investitionsmodell, dem der idealtypische Verlauf eines Innovationsprozesses im sinne einer strukturvorhersage gegenübergestellt wird. Aus dem Ver- 
gleich dieser beiden Ansätze werden drei Problembereiche abgeleitet, die für Innovationsentscheidungen von besonderer Bedeutung sind. Im folgenden wurden deshalb die Wirkungen der Wettbewerbsbedingungen auf das Innovationsverhalten sowie der Einfluß der Besteuerung und der Finanzierungsbedingungen auf die Risiko- und Innovationsbereitschaft analysiert.

In Rapitel $D$ wurden zunächst die Eigenschaften der Innovationskostenfunktion in Abhängigkeit von der Dauer des Innovationsprozesses diskutiert. Aufgrund der Eigenschaften von Forschungs- und Entwicklungsprozessen als Verfahren des Trialand-Error wird eine Funktion mit überproportionalem Kostenanstieg bei einer Verkürzung des Innovationszeitraums unterstellt.

Kann der Innovator eine Quasi-Rente abschöpfen, dann lautet sein Innovationskalkül im Optimum ohne die Berücksichtigung von Konkurrenzbeziehungen, daß der Anstieg des Barwerts der Erträge durch eine beschleunigte Realisierung der Innovation gleich dem Anstieg der Kosten durch die Verkürzung des Innovationszeitraums sein muß.

Auf diesem Kalkül basiert auch der entscheidungstheoretische Ansatz, in dem Ronkurrenzbeziehungen berücksichtigt werden. Drei mögliche Ertragskonstellationen werden in diesem Modell unterschieden. Zum einen kann das betreffende Unternehmen Innovator sein und die gesamte Rente abschöpfen, zum zweiten kann es zwar Innovator sein, seine Innovatoren-Rente wird aber durch Imitatoren geschmälert, und zum dritten kann es selbst Imitator sein. Uber den Eintritt dieser Ronstellationen bildet sich das Unternehmen subjektive Wahrscheinlichkeiten. Die Modellanalyse zeigt, daß eine Intensivierung des Innovationswettbewerbs $z u$ einer Beschleunigung der Innovationsanstrengungen führt. Ubersteigt die Innovationsintensität jedoch einen kritischen Wert, wird der Erwartungswert des Innovationsertrags negativ und eine Position des Abwartens wird lohnend. Bei vollkommenem Wettbewerb resultiert das bekannte Ergebnis, daß die Innovationstätigkeit zum Erliegen kommt. während in diesem entscheidungstheoretischen Ansatz ein optimaler Ausgabenplan bei gegebenen Annahmen über den Eintritt von Ronkurrenten in den Markt abgeleitet wird, ist im spieltheoretischen Modell der Markteintritt von den Forschungs- 
und Entwicklungsaufwendungen abhängig. In einem Patentrennen konkurrieren $n$ Unternehmen um die Innovatoren-Rente. Je höher die Wettbewerbsintensität wird, desto stärker werden die Innovationsanstrengungen forciert. Je höher die Anzahl der Marktteilnehmer ist, desto früher liegt in einem cournotNash-Gleichgewicht der Innovationszeitpunkt.

Der Innovationszeitpunkt wird jedoch in beiden Modellen nicht so weit vorverlagert, daß im Wettbewerb der Innovatoren der Wohlfahrtseffekt der Innovation negativ wird.

Gegen die Tendenz zur vorzeitigen Realisierung von Innovationen im Wettbewerb wirken die mit der Forschungs- und Entwicklungstätigkeit verbundenen externen Effekte. Können sich die Unternehmen nicht alle Erträge ihrer Aktivitäten aneignen, so reduzieren sie ihre Anstrengungen.

Inwieweit bei gegebener Marktstruktur und bei den bestehenden patentrechtlichen Institutionen ein Pareto-optimum erreicht wird, kann deshalb - sofern es gelingt, die erforderlichen Informationen $z u$ beschaffen - jeweils nur im konkreten Einzelfall beurteilt werden.

Gegenstand von Rapitel $\mathrm{E}$ waren die Wirkungen der Besteuerung auf die Bereitschaft zur Risikoübernahme. Zunächst wurde in einem Portefeuille-Modell die Fragestellung untersucht, wie durch die Erhebung einer Ertragsteuer die Verteilung des anlagefähigen Vermögens auf risikolose und risikobehaftete Anlageformen beeinflußt wird. Eine eindeutige Besteuerungswirkung kann für den Fall einer ertraglosen risikofreien Anlage abgeleitet werden. Die Einführung einer Ertragsteuer führt zu einer Vermögensumschichtung zugunsten der risikobehafteten Anlage, um das vor steuern realisierte Erwartungsnutzenniveau wieder zu erreichen. Bringt jedoch auch die risikolose Anlageform einen Ertrag, so tritt neben den substitutionseffekt ein Vermögens- oder Einkommenseffekt und eindeutige Besteuerungswirkungen können nicht bestimmt werden. Der Effekt der Besteuerung auf die Bereitschaft zur Obernahme von Risiken ist abhängig von der Vermögenselastizität der Nachfrage nach risikobehafteten Anlageformen.

Um zusätzliche Fragestellungen analysieren zu können, wurde ein zweiter, direkter Ansatz zur Untersuchung der Besteuerungswirkungen auf die Risikoübernahmebereitschaft einge- 
führt. Für die Bestimmung der Risikowirkungen sind drei Partialeffekte zu unterscheiden: der Jensen-Effekt, die Residuale Einkommensprogression und die Relative Risikoaversion. Die Anwendung dieses Instrumentariums zeigt, daß für allgemeine steuerliche Regelungen, wie zum Beispiel steuertarife, keine eindeutigen Wirkungen auf die Risikobereitschaft abgeleitet werden können. Aussagen über die Richtung der Risikowirkung können höchstens für einzelne Bereiche bei Renntnis der individuellen Risikonutzenfunktionen gemacht werden. Eine Ausnahme bilden diskriminierende steuerliche Regelungen, die risikolose bzw. risikobehaftete Einkommen bevorzugen oder benachteiligen. Ein Beispiel hierfür sind Verlustausgleichsregelungen, die einseitig risikobehaftete Einkommen betreffen.

Eine Dämpfung der Risikobereitschaft wird bei risikoaversem Entscheidungsverhalten der Wirtschaftssubjekte durch die von der Steuerpolitik erzeugte Unsicherheit verursacht. Häufige Gesetzesänderungen, inexakte Rechtsetzung und die damit verbundenen Anpassungsprozesse erhöhen das Risiko wirtschaftlicher Betätigung und lassen substitutionsbewegungen zugunsten risikoloser Engagements erwarten.

In Kapitel $\mathrm{F}$ wurde der dritte Problembereich, der Einfluß der Finanzierungsbedingungen auf die Innovationsaktivitäten untersucht. Das Kapitel ist in zwei Teile gegliedert. Im ersten werden die steuerlichen Bedingungen der Kapitalbildung sowie die steuerliche Behandlung alternativer Finanzierungsformen analysiert. Im zweiten Teil erfolgt eine Diskussion der Möglichkeiten neugegründeter sowie kleiner und mittlerer Unternehmen Rapital zur Finanzierung von Innovationen zu beschaffen.

Die Rapitalbildung wird auf zwei Ebenen von steuerlichen Institutionen beeinflußt. Einerseits wird die Höhe des anlagefähigen Betrags durch das Ausmaß der Abgabenbelastung mit bestimmt. Zum zweiten beeinflußt die steuerliche Behandlung der Kapitalerträge Richtung und Volumen der Rapitalakkumulation. Im Bereich der Rapitalbildung privater Haushalte können Beiträge an Bausparkassen und bestimmte Versicherungen bei der Einkommensermittlung als Sonderausgaben abgezogen werden. Das Rapital wird somit aus unversteuertem Einkommen gebildet. Zudem bleiben die auf diese Beträge anfallenden Rapitalerträge 
steuerfrei. Diese steuerlichen Regelungen werden ergänzt durch Leistungen nach dem Wohnungsbau-Prämiengesetz, dem Spar-Prämiengesetz und dem Fünften Gesetz zur Förderung der Vermögensbildung der Arbeitnehmer. In die begünstigten Anlageformen sind nach den beiden letztgenannten Gesetzen auch Beteiligungstitel an Unternehmen einbezogen. Der Kreis der Begünstigten ist allerdings auf steuerpflichtige mit zu versteuernden Einkommen von unter 24.000 DM bzw. 48.000 DM bei zusammenveranlagten Ehegatten beschränkt. Die Bildung von Gründungskapital wird durch diese staatlichen Transferleistungen nur unwesentlich begünstigt.

Das Selbstfinanzierungspotential von Unternehmen wird durch die steuerliche Belastung der einbehaltenen Gewinne bestimmt. Bei Kapitalgesellschaften beträgt allein die ertragsteuerliche Belastung bei einem Hebesatz der Gewerbesteuer von 350 Prozent 62,55 Prozent des Thesaurierungsbetrags. Dies bedeutet, daß nur etwa ein Drittel des Bruttogewinns zur Finanzierung weiterer Aktivitäten zur Verfügung steht.

Die Analyse der steuerlichen Behandlung alternativer Finanzierungsformen ergab eine eindeutige steuerliche Präferenz für die Fremdfinanzierung. Grund dafür sind die unvollständige Hinzurechnung der Dauerschuldzinsen bzw. der Dauerschulden zur Bemessungsgrundlage der Gewerbesteuer sowie die vermögensteuerliche Behandlung des Fremdkapitals als schuldposten. Nur bei relativ niedrigen Gewinnen und überschaubarem Gesellschafterkreis kann durch Ausschüttung und anschließende Wiedereinlage die Eigenfinanzierung unter Ausnutzung des progressiven Einkommensteuertarifs vorteilhaft werden. Allerdings reduziert die bei Beteiligungsfinanzierung anfallende Gesellschaftsteuer diesen Vorteil.

Bei der Analyse der Rapitalbeschaffungsmöglichkeiten für Innovationsprojekte wurde zunächst der Zugang der Unternehmen zu Fremakapital untersucht. Die Banken sind in der Bundesrepublik Deutschland die wichtigsten Vermittler von Fremdkapital. Werden die Rreditausfallquoten als Indikatoren der Risikobereitschaft bei der Rreditvergabe verwendet, so zeigt sich, daß größere Risiken von den Banken nicht übernommen werden. Bei solchem risikoaversen Entscheidungsverhalten sind die Möglichkeiten der Fremdinanzierung riskanter Projekte durch die zur Verfügung stehenden Sicherheiten begrenzt. Die- 
se sind jedoch bei neugegründeten sowie kleinen und mittleren Unternehmen nur in geringerem Umfang vorhanden, so daß diese auf die Beschaffung von Beteiligungskapital angewiesen sind. Die Eigenfinanzierung über den Aktienmarkt erweist sich zwar als der kostengünstigste Weg der Beschaffung von Beteiligungskapital, die mangelnde hierarchische Abstufung der einzelnen Börsensegmente nach dem Anlagerisiko versperrt aber kleinen und mittleren Unternehmen den zugang zum Rapitalmarkt. Zudem ist bei der Umwandlung in eine Aktiengesel1schaft die Errichtung eines mitbestimmten Aufsichtsrats unabhängig von der Unternehmensgröße vorgeschrieben. Diese Regelung stellt ein zusätzliches Hemmnis für die Beschaffung von Eigenkapital über den Kapitalmarkt durch neue, kleine und mittlere Unternehmen dar.

Auf die Untersuchung der einzelwirtschaftlichen Grundlagen sowie des Einflusses der Besteuerung und der Finanzierungsbedingungen auf die Innovationsbereitschaft folgt im dritten Teil der Arbeit die Analyse der Möglichkeiten und Grenzen einer marktwirtschaftichen Innovationspolitik in einem demokratischen system.

In Rapitel $G$ wurde zunächst die Reichweite der Innovationspolitik definiert. Die innovationspolitischen Aktivitäten umfassen alle staatlichen Maßnahmen auf einzelwirtschafticher Ebene, die Ausmaß und Richtung der Innovationsanstrengungen der privaten Wirtschaftssubjekte beeinflussen. Diese Definition schließt als Mittel der Innovationspolitik die fiskalischen Instrumente sowie die regulierenden Interventionen ein. Notwendige Bedingung für wirtschaftspolitische Eingriffe in einer marktwirtschaftlichen ordnung ist das vorliegen von Marktversagen. Die Analyse der notwendigen Bedingung zeigt, daß für einen wirkungsvollen Einsatz der wirtschaftspolitischen Instrumente beträchtliche Informationsprobleme zu lösen sind. Zudem tut sich bei der Förderung von Innovationen ein logischer widerspruch auf. Für eine induzierend, d.h. veranlassend gedachte Politik muß der Fördergegenstand bekannt sein. Innovationen sind aber neuartige, d.h. bis dato unbekannte Kombinationen von Produkten bzw. Produktionsverfahren und Nachfrage. Somit kann eine induzierende Innovationsförde- 
rung die notwendige Bedingung nicht erfüllen.

Weitere Einschrånkungen der innovationspolitischen Möglichkeiten ergeben sich aus der hinreichenden Bedingung für wirtschaftspolitische Eingriffe. Eine wirtschaftspolitische Intervention ist nur vorzunehmen, wenn das Marktergebnis nach erfolgtem Eingriff und dem Abschluß der durch ihn ausgelösten Anpassungsreaktionen günstiger als das unkorrigierte Marktergebnis ist. Jede begünstigende Maßnahme führt zu einer Ungleichbehandlung ursprünglich ökonomisch gleichgestellter wirtschaftssubjekte. Diese Diskriminierung löst marktliche und politisch-ökonomische Reaktionen aus, die dieser Ungleichbehandlung entgegenwirken und damit aber auch die wirksamkeit der Maßnahme reduzieren oder aufheben. Solche Kapitalisierungsprozesse erzeugen neuen wirtschaftspolitischen Handlungsbedarf. Eine Akkumulation von Eingriffen mit wachsender Intransparenz und zunehmenden Informationsproblemen sowie massiven störungen des marktlichen Anreizsystems ist die Folge. Eine Vernachlässigung dieser Anpassungsprozesse verursacht somit beträchtliche volkswirtschaftliche Rosten.

Die Analyse der notwendigen und der hinreichenden Bedingung für wirtschaftspolitische Eingriffe ergab, daß einer induzierend, d.h. einer veranlassend angelegten Politik, die bestimmte Innovationsaktivitäten der privaten wirtschaftssubjekte fördert, die erforderlichen Grundlagen fehlen. Wie die Diskussion des Ablaufs von Innovationsprozessen zeigte, sind jedoch Aussagen über die strukturen von Innovationsaktivitäten möglich. Aufbauend auf solchen strukturvorhersagen im Sinne Hayeks wurde das Konzept einer akkommodierenden Innovationspolitik entwickelt.

Ausgangspunkt ist das Modell einer dynamischen Wirtschaft. Triebfeder der ökonomischen Entwicklung ist das Bestreben der Unternehmen, durch das Ausfüllen von Koordinationslücken zwischen den individuellen Planungen der Wirtschaftssubjekte Gewinnerzielungsmöglichkeiten auszuschöpfen. Unterscheiden lassen sich dabei Innovations- und Arbitrageaktivitäten. Während letztere auf den Ausgleich zeitpunktbezogener Ungleichgewichte ausgerichtet sind, sollen durch die Innovationsanstrengungen zeitraumbezogene Roordinationslücken durch neuartige Angebote geschlossen werden. Um Innovationsaktivitäten zu för- 
dern, sind deshalb im Rahmen einer akkommodierenden Innovationspolitik Diskriminierungen risikobehafteter, unternehmerischer Aktivitäten zu beseitigen, Erhöhungen des unternehmerischen Risikos durch wirtschaftspolitische Maßnahmen zu verhindern, sowie Kapital- und Gütermärkte für neuartige Vorhaben offenzuhalten.

Anhand der in den voranstehenden Rapiteln entwickelten Kriterien wurden dann in Kapitel $\mathrm{H}$ die Innovationsaktivitäten in der Bundesrepublik Deutschland kritisch untersucht. Aus der Zusammenstellung der ziele und Grundsätze der Innovationspolitik läßt sich keine einheitliche Ausrichtung der Politik ableiten. Zwar erfolgt regierungsamtlich eine grundsätzliche Festlegung auf eine ordnungspolitisch orientierte Politik, gleichzeitig werden jedoch aus verschiedenen Gründen innovationspolitisch motivierte, selektive Eingriffe gerechtfertigt.

Externe Effekte kōnnen nur im Bereich der Grundlagenforschung, nicht aber in der angewandten Forschung und Entwicklung staatliche Interventionen begründen. Zum einen können im Bereich von angewandter Forschung und Entwicklung externe Effekte durch die geeignete Ausgestaltung des gewerblichen Rechtschutzes internalisiert werden. Auch ermöglicht die Erfordernis eines entsprechenden Know-how die Aneignung der Erträge dieser Aktivitäten durch den Innovator. Zum zweiten setzt der Wettbewerb auf Märkten mit niedrigen Marktzugangsschranken Innovationsanreize.

Aus der Tatsache, daß im Marktprozeß nur aktuelle Nachfrage und aktuelles Angebot aufeinander abgestimmt werden, kann ebenfalls kein Marktversagen abgeleitet werden. Die am Markt explizit werdenden Präferenzen und Aktivitäten der Wirtschaftssubjekte sind Teile ihrer Gesamtplanungen und schlieBen Erwartungen über die zukünftigen umweltzustände mit ein. Ober diese individuellen Wirtschaftspläne werden somit Vorstellungen über die weitere Entwicklung von Angebots- und Nachfragebedingungen in den Abstimmungsproze $\beta$ am Markt hineingetragen.

Obersteigt der Ressourcenbedarf für ein Innovationsprojekt das Finanzierungspotential eines einzelnen Unternehmens, so ist dies nicht gleichbedeutend mit einem Verzicht auf dieses 
Vorhaben. Sind nämlich die Gewinnerwartungen größer als die Roordinationskosten, können durch Unternehmenskooperationen die erforderlichen Mittel bereitgestellt werden. Aus Unteilbarkeiten kann bei entsprechender ökonomischer Attraktivität der Projekte nicht auf ein Marktversagen geschlossen werden, zumal staatliche Eingriffe auch nicht ohne Rosten erfolgen.

Neben die verschiedenen Argumente zum Marktversagen treten Fördermaßnahmen anderer Länder für konkurrierende Industriezweige zur Begründung eines staatlichen Interventionsbedarfs. Bei der Bestimmung des Subventionsbedarfs zur Rompensation von Vorteilen der ausländischen Ronkurrenten zeigt sich aber, daß eine solche Politik nur in einzelnen Fällen einen positiven Erwartungswert hat. Werden zudem die Anpassungsreaktionen in die Analyse einbezogen, die durch die begünstigenden Maßnahmen ausgelöst werden, so erscheint ein Verzicht auf die Teilnahme am internationalen subventionswettbewerb ökonomisch rational.

Bei den staatlichen Ausgaben zur Förderung von Forschung und Entwicklung in der gewerblichen Wirtschaft der Bundesrepublik Deutschland dominiert die direkte Projektförderung. Wie aus den obigen Ausführungen hervorgeht, kann eine solche induzierende Politik ökonomisch nicht überzeugend begründet werden. Zusätzlich wirft diese Vorgehensweise neue Probleme auf. Zum einen sind bei dieser Art der Förderung die wirtschaftlichen Rontrollanreize gering. Fördernde Institutionen und geförderte Unternehmen haben kein Interesse am Abbruch eines unrentablen Projekts, da dieser als offentliches Eingeständnis einer Fehlentscheidung gewertet werden könnte. Deshalb werden insbesondere staatliche Prestigeobjekte häufig bis zum technischen Erfolg weitergeführt, auch wenn keine Möglichkeit zur wirtschaftichen Verwertung der technischen Neuerung gegeben sein sollte. Zum anderen werden durch die direkte Projektförderung vor allem Großunternehmen begünstigt. Um der Diskriminierung kleiner und mittlerer Unternehmen entgegenzuwirken, wurde seit der zweiten Hälfte der siebziger Jahre eine Vielzahl von Programmen zugunsten dieser Unternehmen initiiert. Die Benachteiligung kleiner und mittlerer Unternehmen durch die bestehenden Förderprogramme sowie die zugangshindernisse insbesondere auf dem Kapitalmarkt können jedoch keine staatlichen Ausgleichsmaßnahmen im Sinne der Theorie des Zweitbe- 
sten begründen. Die aufgeführten Innovationshemmnisse sind durch staatlich gesetzte Institutionen verursacht. Die geeignete Politik besteht deshalb in der Beseitigung dieser störungen und nicht in der Vornahme zusätzlicher Eingriffe.

Außerdem erfolgt durch die staatlichen Fördermaßnahmen eine Diskriminierung zwischen den verschiedenen Bereichen der Innovationstätigkeit, da die staatliche Förderung nur auf technische Neuerungen abzielt und andere Innovationsaktivitäten vernachlässigt.

Die Analyse des Einkommensteuertarifs fūr 1990 bestãtigt die in Rapitel E konstatierte Skepsis bezüglich der Möglichkeiten einer zielgerichteten Risikopolitik. Wie dort bereits ausgeführt, sind die Risikowirkungen der Tarifänderungen von den individuellen Risikopräferenzen und dem Einkommensniveau bzw. der jeweiligen Alternative abhängig.

Da die Tarifänderung eine durchgehende steuerliche Entlastung bringt, erhöht sie die Gewinnanreize und reduziert durch die Verschärfung der Budgetrestriktion die Möglichkeiten von Interessengruppen im politischen Prozeß. Zudem wird der Druck zur Oberprüfung von Ausnahmetatbeständen erhöht.

Rritisch zu beurteilen ist die geplante senkung des Thesaurierungssatzes der Körperschaftsteuer unter den spitzengrenzsteuersatz der Einkommensteuer. Diese Maßnahme führt zu neuen Verzerrungen zwischen Rechtsformen und zwischen Finanzierungsinstrumenten und bewirkt eine Abschwächung der selektionsfunktion des Marktes.

Die im weiteren untersuchte Einführung eines neuen Marktabschnitts an der Börse führt nur dann zu einer Erleichterung der Eigenfinanzierung kleiner und mittlerer Unternehmen über den Kapitalmarkt, wenn durch die Ausgestaltung der Zugangsbedingungen $z u$ den einzelnen Börsensegmenten auch eine Abstufung der Teilmärkte nach dem Anlagerisiko erzielt wird. Die Konkretisierung der Zulassungsbedingungen in den Börsenordnungen deutet bislang nicht darauf hin, daß durch die gesetzlichen Maßnahmen eine Verbesserung der Eigenfinanzierung kleiner und mittlerer Unternehmen erreicht werden kann.

An den Oberblick über die innovationspolitischen Aktivitäten in der Bundesrepublik Deutschland schließen sich Vorschläge für Maßnahmen im Rahmen einer akkommodierenden Innovationspolitik an. Sie wurden aus dem in Kapitel $G$ vorgestellten Kon- 
zept abgeleitet und zielen auf die Beseitigung von diskriminierenden Regelungen und Beschränkungen des Marktzugangs ab. Im einzelnen wurden im Bereich der steuerpolitik eine Neuregelung der steuerlichen Verlustkompensation sowie Anderungen zur Beseitigung von steuerlichen Diskriminierungen zwischen Finanzierungsformen bzw. zwischen Rechtsformen vorgeschlagen. Im Rahmen der Rapitalmarktpolitik wurde eine systematische offnung der Rapitalmārkte für neugegründete sowie kleine und mittlere Unternehmen zur Finanzierung innovativer, risikobehafteter Projekte angeregt. Industriepolitische Maßnahmen sind so auszugestalten, daß sie keine lenkenden oder marktabschließenden Wirkungen haben. Sind zur Erfüllung öffentlicher Aufgaben Forschungs- und Entwicklungsarbeiten erforderlich, so sind diese als entsprechend spezifizierte Forschungsaufträge zu vergeben. 


\section{LITERATURVERZEICHNIS}

Aaron, H.J. (Hrsg.)

Inflation and the Income Tax, Washington, D.C. 1976

Abernathy, W.J., Utterback, J.M.

Patterns of Industrial Innovation, in: Readings in the Management of Innovation, M.L. Tushman und W.L. Moore (Hrsg.), Boston u.a.0. 1982, S. 97-108

Ahsan, S.M.

Progression and Risk-Taking, in: Oxford Economic Papers $26(1974)$, S. $318-328$

Ahsan, S.M.

Taxation in a Two-Period Temporal Model of Consumption and Portfolio Allocation, in: Journal of Public Economics $5(1976)$, S. 337-352

Akerlof, G.A.

The Market for "Lemons": Quality Uncertainty and the Market Mechanism, in: Quarterly Journal of Economics 84(1970), S. 488-500

Albach, H., Hunsdiek, D., Kokalj, L. Finanzierung mit Risikokapital, stuttgart 1986

Allen, R.G.D.

Mathematische wirtschaftstheorie, Berlin 1971

Allingham, $G$.

Risk-Taking and Taxation, in: Zeitschrift für Nationalökonomie $32(1972)$, S. 203-224

Arbeitsgruppe steuerreform

steuern der neunziger Jahre - Leitlinien für eine Reform -, stuttgart 1987

Arrow, $\boldsymbol{R} . J$.

Economic Welfare and the Allocation of Resources for Invention, in: Economics of Information and Rnowledge, D.M. Lamberton (Hrsg.), Hammondsworth 1971a, S. 141-159 
Arrow, $\boldsymbol{K} . J$.

Essays in the Theory of Risk-Bearing, Amsterdam und London 1971b

Atkinson, A.B., stiglitz, J.E.

Lectures on Public Economics, Maidenhead 1980

Averch, H., Johnson, L.L.

Behavior of the Firm under Regulatory Constraint, in: American Economic Review 52(1962), S. 1052-1069

Baily, M.N.

Productivity and the Services of Capital and Labor, in: Brookings Papers of Economic Activity 1(1981), S. 1-50

Baily, M.N.

Will Productivity Growth Recover? Has it Done so Already?, in: American Economic Review 74(1984), S. 231-235

Baily, M.N.

The Productivity Growth slowdown by Industry, in: Brookings Papers on Economic Activity 2(1985), S. 423-458

Baily, M.N., Chakrabarti, A.R.

Innovation and Productivity in U.S. Industry, in: Brookings Papers on Economic Activity 2(1985), S. 609-639

Bain, J.S.

Barriers to New Competition, Cambridge 1956

Bamberg, G., Richter, W.F.

The Effects of Progressive Taxation on Risk-Taking, in: Zeitschrift für Nationalökonomie 44(1984), S. 93-102

Bamberg, G., Richter, W.F.

Risk-Taking under Progressive Taxation: Three Partial Effects, Diskussionsbeitrag, Augsburg und Dortmund 1985

Bamberg, G., Richter, W.F.

Decision-Theoretic Analysis of Envisaged Income Tax Reforms for FRG and U.S., Diskussionsbeitrag, Augsburg 1986 
Bamberg, G., Spremann, $\boldsymbol{K}$.

Implications of Constant Risk Aversion, in: Zeitschrift für Operations Research 25(1981), S. 205-224

Bareis, $P$.

Reform der Rörperschaftsteuer: Steuerbereinigungen und Vereinfachungen, in: Steuerberaterkongreß-Report 1987, S. 33-88

Barze1, $Y$.

Optimal Timing of Innovations, in: Review of Economics and statistics $50(1968)$, S. 348-355

Bauer, $J$.

Grundlagen einer handels- und steuerrechtlichen Rechnungspolitik der Unternehmung, Wiesbaden 1981

Becker, G.S.

A Theory of Competition among Pressure Groups for Political Influence, in: Quarterly Journal of Economics 98(1983), S. $371-400$

Berndt, $\boldsymbol{B} . \boldsymbol{R}$.

Energy Price Increases and the Productivity Slowdown in United States Manufactoring, in: The Decline in Productivity Growth, Federal Reserve Bank of Boston (Hrsg.), Boston 1980, S. $60-89$

Bierich, $\boldsymbol{M}$.

Der Einfluß der Besteuerung auf die Rapitalbeschaffung der Unternehmen, in: Zeitschrift für betriebswirtschaftliche Forschung $35(1983)$, S. 41-60

Bittker, B.J.

Tax Shelters and Tax Capitalization or Does the Early Bird Get a Free Lunch?, in: National Tax Journal 28(1975), S. 416-419

Blume, M.B., Friend, I.

The Asset Structure of Individual Portfolios and some Implications for Utility Functions, in: Journal of Finance $30(1975)$, S. 585-603 
Boadway, R.W.

Public Sector Economics, Cambridge, Mass. 1979

Boadway, R.W., Wildasin, D.B.

Public Sector Economics, 2. Aufl., Boston und Toronto 1984

Booz, Allen and Hamilton Inc.

Management of New Products, 4. Aufl., New York 1964

Borell, R., Schemmel, L.

Steuervereinfachung, in: Deutsche steuerzeitung $21(1987), \mathrm{S}$. 110-116, S. 142-147, S. 191-195, S. 250-254

Brennan, G., Buchanan, J.M.

Predictive Power and the Choice among Regimes, in: Economic Journal 93(1983), S. 89-105

Brennan, G., McGuire, T.

Optimal Policy Choice under Uncertainty, in: Journal of Public Economics 4(1975), S. 205-209

Bruno, M.

Raw Materials, Profits, and the Productivity slowdown, in: Quarterly Journal of Economics 99(1984), S. 1-29

Buchanan, J.M.

Taxation in Fiscal Exchange, in: Journal of Public Economics $6(1976)$, S. 17-29

Buchanan, J.M.

Introduction, in: Towards a Theory of the Rent-Seeking Society, J.M. Buchanan, R.D. Tollison und G. Tullock (Hrsg.), College Station 1980 , S. 3-15

Buchholz, W.

Die Wirkungen progressiver steuern auf die Vorteilhaftigkeit riskanter Investitionen, in: Zeitschrift für betriebswirtschaftliche Forschung $37(1985)$, S. 882-890

Bundesminister für Forschung und Technologie/Bundesminister für Wirtschaft

Forschungs- und technologiepolitisches Gesamtkonzept der Bundesregierung für kleine und mittlere Unternehmen, Bonn 1978 
Bundesminister für Forschung und Technologie

Bundesbericht Forschung 1984, Bonn 1984

Bundesminister für Forschung und Technologie (Hrsg.)

Ratgeber Forschung und Technologie 1986, Köln 1986

Bundesminister für wissenschaftliche Forschung

Bundesforschungsbericht III, Bonn 1969

Bundesministerium der Finanzen (Hrsg.)

Zehnter Subventionsbericht, Bonn 1985

\section{Bundesregierung}

Antwort auf Große Anfrage betr. Neuausrichtung der Forschungs- und Technologiepolitik in der Bundesrepublik Deutschland, Bundestags-Drucksache 10/710, Bonn 1983

\section{Bundesregierung}

Faktenbericht 1986 zum Bundesbericht Forschung, BundestagsDrucksache 10/5298, Bonn 1986

Börner, D., Rrawitz, N.

Steuerbilanzpolitik, Herne und Berlin 1977

Cansier, $D$.

Steuern und Investitionsrisiko, in: offentliche Finanzen, Rredit und Rapital, D. Cansier und D. Kath (Hrsg.), Berlin 1985, S. 255-283

Capitelli, R., Mūller, H.H.

Ein Beispiel zum Problem der Innovationsförderung, in: Schweizerische Zeitschrift für Volkswirtschaft und statistik $122(1986)$, S. 535-553

Chaney, P.K., Thakor, A.V.

Incentive Effects of Benevolent Intervention, in: Journal of Public Economics 26(1985), S. 169-189

Christiansen, G.B., Haveman, R.H. Public Regulations and the slowdown in Productivity Growth, in: American Economic Review, Papers and Proceedings, $71(1981)$, S. 320-325 
Clapham, R., Scholz-Bappert, D.E.

Technologieparks - Ziele, Instrumente, Wirkungen -, Diskussionsbeitrag, Siegen 1986

Clark, P.R.

Capital Formation and the Recent Productivity slowdown, in: Journal of Finance $33(1978)$, S. 365-375

Clark, P.R.

Inflation and the Productivity Decline, in: American Economic Review, Papers and Proceedings, 72(1982), S. 149-154

Clark, P.R.

Productivity and Profits in the 1980s: Are They Really Improving?, in: Brookings Papers on Economic Activity 1(1984), S. 133-167

Claussen, C.P.

Der neue Zweite Markt, in: Zeitschrift für Unternehmens- und Gesellschaftsrecht $13(1984)$, S. 1-22

Cohn, R.A. et al.

Individual Investor Risk Aversion and Investment Portfolio Composition, in: Journal of Finance 30(1975), S. 605-620

Darby, M.N.

The U.S. Productivity Slowdown: A Case of Statistical Myopia, in: American Economic Review 74(1984), S. 301-322

Dasgupta, P., stiglitz, J.B.

Industrial structure and the Nature of Innovative Activity, in: Economic Journal 90(1980), S. 266-293

Delbrūck, J.

"Kapitalmarktreife" - Der Unternehmer und der Schritt in den Aktienmarkt -, in: Venture Capital für junge Technologieunternehmen, E. Heilmayr (Hrsg.), Haar bei München 1983, S. 141-147

Demsetz, $H$.

Information and Efficiency: Another Viewpoint, in: Journal of Law and Economics $12(1969)$, S. 1-22 
Denison, E.F.

Accounting for United States Economic Growth, 1929-1969, Washington, D.C. 1964

Denison, E.F.

Accounting for Slower Economic Growth: The United States in the 1970s, Washington, D.C. 1979

Diamond, P.A., Stiglitz, J.B.

Increases in Risk and Risk Aversion, in: Journal of Economic Theory 8(1974), S. 337-360

Dicke, H., Hartung, H.

Externe Rosten von Rechtsvorschriften. Möglichkeiten und Grenzen ökonomischer Gesetzesanalyse, Tübingen 1986

Dittes, $B$.

Die Finanzierungshilfen des Bundes und der Länder für die gewerbliche Wirtschaft, in: Zeitschrift für das gesamte kreditwesen, Sonderausgabe, 40 (1987)

Domar, B.D., Musgrave, R.A.

Proportional Income Taxation and Risk-Taking, in: Quarterly Journal of Economics 58(1944), S. 388-422

Downs, A.

Okonomische Theorie der Demokratie, Tübingen 1968

Drukarczyk, J.

Finanzierung, 2., überarbeitete Auf1., Stuttgart und New York 1985

Dreze, J.H., Modigliani, F.

Consumption Decisions under Uncertainty, in: Journal of Economic Theory 5(1972), s. 308-335

Dürr, $E$.

Alternativen der Strukturpolitik, in: Jahrbuch für Sozialwissenschaft $35(1984)$, S. 402-412

Eisenmann, H.

Grundriß Gewerblicher Rechtschutz und Urheberrecht, Heidelberg 1985 
Ekern, $S$.

Taxation, Political Risk and Portfolio selection, in: Economica $38(1971)$, S. 421-430

Ert1, B.

Die Bundesrepublik - Entwicklungsland auf dem Rapitalmarkt? Möglichkeiten zur Aktivierung des Neuemissionsmarktes, in: Venture Capital für junge Technologieunternehmen, E. Heilmayr (Hrsg.), Haar bei München 1983, S. 135-139

Euba, $\boldsymbol{N}$.

Ziele und Wirksamkeit staatlicher Gewerbeförderung dargestellt am Beispiel Baden-Württemberg, in: Offentliche Finanzen, Rredit und Kapital, D. Cansier und D. Kath (Hrsg.), BerIin 1985 , s. 305-320

Farrar, D.E., Selwyn, L.L.

Taxes, Corporate Financial Policy and Return to Investors, in: National Tax Journal 20(1967), S. 444-454

Feldstein, M.S.

Compensation in Tax Reform, in: National Tax Journal $29(1976)$, S. 123-130

Feldstein, M.S.

The Effects of Taxation on Risk Taking, in: Journal of Political Economy $77(1969 b), S .755-764$

Feller,

An Introduction to Probability Theory and Its Applications, Bd. 2, 2. Aufl., New York u.a.0. 1971

Fellingham, J.C., Wolfson, M.A.

The Effects of Alternative Income Tax structures on Risk Taking in Capital Markets, in: National Tax Journal 31(1978), s. $339-347$

Fethke, G.C., Birch, J.J.

Rivalry and the Timing of Innovation, in: Bell Journal of Economics $13(1982)$, S. 272-279 
Folkers, $C$.

Der Reformakzelerator, in: Wirtschaftsdienst 58(1978), S. 340-345

Folkers, $C$.

Zu einer positiven Theorie der steuerreform, in: staatsfinanzierung im Wandel, H.-K. Hansmeyer (Hrsg.), Berlin 1983, S. 189-211

Folkers, $c$.

Steuerreforminteressen und steuervergünstigungen in positiver und institutioneller Perspektive, in: Jahrbuch für Sozialwissenschaft $36(1985)$, S. 274-301

Folkers, $c$.

Die politische Okonomie der Besteuerung, Diskussionsbeitrag, Hohenheim 1986

Folkers, $C$.

Wandlungen der Verbrauchsbesteuerung, in: Wandlungen der Besteuerung, K. Häuser (Hrsg.), Berlin 1987, S. 89-212

Folkers, $c$.

Wer wird durch steuervergünstigungen eigentlich begünstigt? $\mathrm{Zu}$ einem Problem der materiellen steuerinzidenz, erscheint in: Finanzarchiv $46(1988)$

Freeman, $C$.

The Determinants of Innovation, in: Futures 11(1979), S. 206-215

Friebe, $s$.

Steuerreform 1990, in: Deutsche steuerzeitung 22(1988), S. 4-17

Friedman, J.H.

Game Theory with Applications to Economics, New York und Oxford 1986

Friedman, $M$.

Nobel Lecture: Inflation and Unemployment, in: Journal of Political Economy 85 (1977), S. 451-472 
Friend, J., Blume, M.E.

The Demand for Risky Assets, in: American Economic Review $65(1975)$, S. 900-922

Friend, J., Howitt, $P$.

Credtit Rationing an Implicit Contract Theory, in: Journal of Money, Credit, and Banking 12(1980), S. 471-487

Gierlichs, H.

Industrieobligationen, in: Handwörterbuch der Finanzwirtschaft, Stuttgart 1976, Sp. 811-818

Giersch, H., Schmidt, H.

Offene Märkte für Beteiligungskapital: USA - Großbritannien Bundesrepublik Deutschland, Stuttgart 1986

Griliches, $z$.

Productivity, $R \& D$, and Basic Research at the Firm Level in the 1970's, in: American Economic Review 76(1986), S. 141-154

Gutberlet, $\boldsymbol{K} .-L$.

Alternative strategien der Forschungsförderung, Tübingen 1984

Gutowski, A.

Bemerkungen zur Theorie des eintrittsverhindernden Preises im oligopol, in: Wirtschaft und Wettbewerb 21(1971), s. 819-827

Haberstock, L.

Der Einfluß der Besteuerung auf Rechtsform und standort, 2. Aufl., Hamburg 1984

Haberstock, L.

Steuerbilanz und Vermögensaufstellung, 2. Aufl., Hamburg 1984

Haegert, L., Kramm, $\boldsymbol{R}$.

Der Einfluß von Ertragsteuern auf die Vorteilhaftigkeit von Investitionen mit unterschiedlichem Risiko, in: Zeitschrift für betriebswirtschaftliche Forschung 27 (1975), S. 69-83

Hagen, $\boldsymbol{K} . \boldsymbol{P}$.

Taxation and Investment Behavior under Uncertainty - A Multiperiod Portfolio Analysis, in: Theory and Decision $1(1970 / 71)$, S. 269-295 
Ha11, R.B., Jorgenson, D.W.

Tax Policy and Investment Behavior, in: American Economic Review 57 (1967), S. 391-414

Hamm, $\boldsymbol{H}$.

Freiheitsbeschränkung durch staatliche struktur- und Forschungspolitik, in: ORDO 30(1979), S. 423-439

Harberger, A.C.

Three Basic Postulates for Applied Welfare Economics: An Interpretive Essay, in: Journal of Economic Literature 9(1971), S. 785-797

Hauff, V., Scharpf, F.W.

Modernisierung der Volkswirtschaft, Köln 1975

Haveman, R.H., Norsworthy, J.R.

Public Regulations and Productivity Growth: An Assessment, Paper presented at the 43 rd Congress of the International Institute of Public Finance, Paris 1987

Hayek, F.A. von

Der Wettbewerb als Entdeckungsverfahren, Riel 1968

Hayek, F.A. von

Die Theorie komplexer Phänomene, Tübingen 1972

Hayek, F.A. von

Die Anmaßung von Wissen, in: ORDO 26(1975), S. 12-21

Heck, $\boldsymbol{H}$.

Gattermann: Reine Verschuldung zur Restfinanzierung der Reform, in: Die Welt, 13.10.1987, S. 11

Helmstådter, $\boldsymbol{B}$.

Strukturlenkung - bedarf der Markt der staatlichen Voraussicht?, in: Strukturpolitik - wozu?, H. Besters (Hrsg.), Baden-Baden 1978, S. 11-24

Herb, F.

Heimliche steuererhöhungen und steuerreformen in der Bundesrepublik Deutschland, Diskussionsbeitrag, Hohenheim 1986 
Hiemenz, U., Weiss, F.D.

Das internationale Subventionskarussel, Diskussionsbeitrag, Kiel 1984

Hoffmann. W. $-D$.

Die inländische steuerbelastung der Genußrechtsfinanzierung, insbesondere für ausländische Kapitalgeber, in: Recht der Internationalen Wirtschaft $31(1985)$, s. 390-400

Hueck, $\boldsymbol{G}$.

Gesellschaftsrecht, 18. Aufl., München 1983

Hunsdiek, D., Kokalj, L.

Beseitigung des Mangels an Risikokapital, in: Kredit und Kapital, Supplement 9(1985), S. 839-867

HWWA - Institut für Wirtschaftsforschung (Hamburg)

Neue Industriepolitik oder stärkung der Marktkräfte?, Hamburg 1986

Institut der Deutschen Wirtschaft

Zahlen zur wirtschaftichen Entwicklung in der Bundesrepublik Deutschland, Köln 1986

Institut für Wirtschaftspolitik an der Universitãt zu Röln, Ifo-Institut für Wirtschaftsforschung München

Hemmnisse und Hilfen für Unternehmensgründungen, Gutachten erstellt im Auftrag des Bundesministers für Wirtschaft, Köln und Mũnchen 1982

Issing, 0 .

Innovationsförderung und Ordnungspolitik, in: List-Forum $10(1979 / 80)$, S. 33-51

Issing, 0 .

Subventionen - Gefahr für die Soziale Marktwirtschaft, in: Mehr soziale Marktwirtschaft - weniger subventionswirtschaft, Ludwig-Erhard-stiftung e.V. Bonn (Hrsg.), stuttgart und New York 1984, S. 3-16

Issing, 0 .

Irrwege europäischer Industriepolitik, in: Volkswirtschaftliche Rorrespondenz der Adolf-Weber-Stiftung 25(1986), Nr. 2 
Jensen, M.C., Meckling, W.H.

Theory of the Firm: Managerial Behavior, Agency Costs and Ownership structure, in: Journal of Financial Economics $3(1976)$, S. 305-360

Jorgenson, D.W.

The Role of Energy in Productivity Growth, in: American Economic Review 74(1984), S. 26-30

Kakwani, N.C.

Applications of Lorenz Curves in Economic Analysis, in: Econometrica $45(1977)$, S. 719-727

Kamien, M.I., Schwartz, N.L.

Timing of Innovations under Rivalry, in: Econometrica $40(1972)$, S. $43-60$

Kamien, M.I., Schwartz, N.L.

Patent Life and R\&D Rivalry, in: American Economic Review $64(1974)$, S. 183-187

Kamien, M.I., Schwartz, N.L.

On the Degree of Rivalry for Maximum Innovative Activity, in: Quarterly Journal of Economics 90(1976), S. 245-260

Kamien, M.I., Schwartz, N.L.

A Generalized Hazard Rate, in: Economics Letters 5(1980), S. 245-249

Kamien, M.I., Schwartz, N.L.

Market structure and Innovation, Cambridge u.a.0. 1982

Kamien, M.I., Tauman, Y.

The Private Value of a Patent: A Game Theoretic Analysis, in: Zeitschrift für Nationalökonomie, Supplementum 4(1984), S. 93-118

Kappe1, $\boldsymbol{R}$.

Internationale Schiffahrt und nationale schiffahrtspolitik empirische und theoretische Bemerkungen zu den Veränderungen in der Weltschiffahrt am Beispiel der Bundesrepublik, in: Beihefte der Ronjunkturpolitik 31(1985), S. 155-204 
Katz, M.L., Shapiro, C.

R\&D Rivalry with Licensing and Imitation, in: American Economic Review 77 (1987), S. 402-420

Raufer, $\boldsymbol{B}$.

Die Okonomik des Patentsystems, in: Industrieökonomik: Theorie und Empirie, G. Bombach, B. Gahlen und A.E. Ott (Hrsg.), Tübingen 1985

Kaufer, $\boldsymbol{B}$.

Forschungs- und Technologiepolitik im Rahmen einer angebotsorientierten Wirtschaftspolitik, in: Wirtschaftspolitik der achtziger Jahre, O. Vogel (Hrsg.), Köln 1982, S. 175-212

Reck, 0 .

West German Science Policy since the early 1960's: Trends and Objectives, in: Research Policy 5(1976), S. 116-157

Reck, 0 .

Government Policy and Technical Choice in the West German Reactor Programme, in: Research Policy 9(1980), S. 302-356

Rendrick, J.W.

Understanding Productivity. An Introduction to the Dynamics of Productivity Change, Baltimore und London 1977

Kendrick, J.W.

Survey of the Factors Contributing to the Decline in U.S. Productivity slowdown, in: The Decline in Productivity Growth, Federal Reserve Bank of Boston (Hrsg.), Boston 1980

Rirzner, I.M.

Competition and Entrepreneurship, Chicago und London 1973, Deutsche Obersetzung: Wettbewerb und Unternehmertum, Tübingen 1978

Rlodt, $\boldsymbol{H}$.

Produktivitätsschwäche in der deutschen Wirtschaft, Tübingen 1984

Rlodt, $\boldsymbol{H}$.

Wettlauf um die zukunft, Tübingen 1987 
Knobbe - Reuk, B.

Bilanz der Rörperschaftsteuerreform 1977, in: GmbH - Rundschau $78(1987)$, S. 125-133

Kohl, H.

Regierungserklärung, in: Bulletin, Presse- und Informationsamt der Bundesregierung (Hrsg.), Nr. 27, Bonn 1987, S. 205-220

Rommission "Zweiter Markt"

Börsenzugang für kleine und mittlere Unternehmen, Ministerium für Wirtschaft, Mittelstand und Technologie (Hrsg.), stuttgart 1987

Rotz, S., Johnson, N.L. (Hrsg.)

Encyclopedia of Statistical sciences, Bd. 4, New York u.a.o. 1983

Rromschrōder, B.

Zum Einfluß der Unsicherheit auf die steuerliche Abschreibungspolitik der Unternehmung, Diskussionspapier, Passau 1983

Kübler, $\boldsymbol{F}$.

Gesellschaftsrecht, Heidelberg und Karlsruhe 1981

Lavoie, $D$.

National Economic Planning: What is Left?, Cambridge, Mass. 1985

Lee, T., Wilde, L.L.

Market structure and Innovation: A Reformulation, in: Quarterly Journal of Economics 94(1980), S. 429-436

Leibenstein, $H$.

X-Efficiency versus Allocative Efficiency, in: American Economic Review $56(1966)$, S. 392-415

Lettenmayer, $s$.

Probleme und Erfahrungen mit der Finanzierung technologischer Innovationen aus der Sicht eines Unternehmens, in: Venture Capital für junge Technologieunternehmen, E. Heilmayr (Hrsg.), Haar bei München 1983, S. 51-58 
Lichtenberg, F.R.

The Relationship between Federal Contract R\&D and Company R\&D, in: American Economic Review 74(1984), S. 73-78

Lichtenberg, F.R.

The Effect of Government Funding on Private Industrial Research and Development: A Re-Assessment, in: Journal of Industrial Economics 36 (1987), S. 97-104

Link, A.N.

Productivity Growth, Environmental Regulation and the Composition of R\&D, in: Bell Journal of Economics 13(1982), S. 548-554

Lipsey, R.G., Lancaster, $R$.

The General Theory of Second Best, in: Review of Economic Studies $24(1956 / 57)$, S. 11-32

Littmann, $\boldsymbol{K}$.

Die Chancen staatlicher Innovationslenkung, Göttingen 1975

Littmann, $\boldsymbol{R}$.

Gewerbesteuern, in: Handbuch der Finanzwissenschaft, F. Neumark (Hrsg.), Bd. 2, 3. Aufl., Tübingen 1980, S. 607-632

Loury, G.C.

Market structure and Innovation, in: Quarterly Journal of Economics 93(1979), S. 395-410

Lüder, $\boldsymbol{K}$.

Zum Einfluß staatlicher Investitionsfördermaßnahmen auf unternehmerische Investitionsentscheidungen, in: Zeitschrift für Betriebswirtschaft 54(1984), S. 531-547

Mas, $C$.

Zur ökonomischen Begründung der Forschungs- und Technologiepolitik, Diskussionsbeitrag, Berlin 1986

Mann, H.

Theorie und Politik der steuerreform in der Demokratie, Frankfurt a.M. U.a.O. 1987 
Mckenzie, R.B.

Competing Visions, Washington, D.C. 1985

Medoff, J.L.

U.S. Labor Markets: Imbalance, Wage Growth, and Productivity in the 1970s, in: Brookings Papers on Economic Activity $1(1983)$, S. 87-120

Meikner, $\%$.

Investitionslenkung, Frankfurt 1974

Menezes, C.F., Hanson, D.L.

On the Theory of Risk Aversion, in: International Economic Review $11(1970)$, S. 481-487

Michels, $R$.

Steuerliche Wahlrechte, Wiesbaden 1982

Ministerium für Wirtschaft, Mittelstand und Technologie Baden-Württemberg (Hrsg.)

Finanzhilfen. Wegweiser zu staatlichen Förderungs- und Finanzierungsmöglichkeiten in Baden-Württemberg, Loseblattsammlung, stand Mai 1987

Ministerium für Wissenschaft und Runst Baden-Württemberg Landesforschungsbericht 1987, stuttgart 1987

Modigliani, F., Miller, M.H.

The Cost of Capital, Corporation Finance, and the Theory of Investment, in: American Economic Review 48(1958), S. 261-297

Modigliani, F., Miller, M.H.

Corporate Income Taxes and the Cost of Capital: A Correction, in: American Economic Review 53(1963), S. 433-443

Mohr, H. - H.

Bestimmungsgründe für die Verbreitung von neuen Technologien, Berlin 1977

Molitor, $B$.

Strukturpolitik aus der sicht des Bundesministeriums für Wirtschaft, in: Beihefte der Ronjunkturpolitik 31(1985), s. $287-303$ 
Morin, R.-A., Fernandez Suarez, A.

Risk Aversion Revisited, in: Journal of Finance 38(1983), S. 1201-1216

Mortensen, D.T.

Property Rights and Efficiency in Mating, Racing, and Related Games, in: American Economic Review 72(1982), S. 968-979

Mossin, $J$.

Taxation and Risk-Taking: An Expected Utility Approach, in: Economica $35(1968)$, S. 74-82

Moxter, A.

Optimaler Verschuldungsumfang und Modigliani-Miller-Theorem, in: Aktuelle Fragen der Unternehmensfinanzierung, K.-H. Forster und P. Schumacher (Hrsg.), Stuttgart 1970, S. 128-155

Müller, J., Vogelsang, $J$.

Staatliche Regulierung: regulated industries in den USA und Gemeinwohlbindung in den wettbewerblichen Ausnahmebereichen in der Bundesrepublik Deutschland, Baden-Baden 1979

Musgrave, R.A.

Finanztheorie, 2. Aufl., Tübingen 1974

Musgrave, R.A., Musgrave, P.B., Kullmer, L. Die offentlichen Finanzen in Theorie und Praxis, Bd. 3, 3. Aufl., Tübingen 1987

Myers, J.G., Nakammura, L.

Energy and Pollution Effects on Productivity: A Putty-Clay Approach, in: New Developments in Productivity Measurement and Analysis, J.W. Rendrick und B.N. Vaccara (Hrsg.), Chicago und London 1980

Nelson, R.R.

Uncertainty, Learning, and the Economics of Parallel Research and Development Efforts, in: Review of Economics and statistics $43(1961)$, S. 351-364

Nelson, $R \cdot R$.

The Role of Knowledge in R\&D Efficiency, in: Quarterly Journal of Economics $97(1982)$, S. 453-470 
Ng, $Y_{0}-\boldsymbol{K}$.

Towards a Theory of Third-Best, in: Public Finance 32(1977), S. $1-15$

Nordhaus, W.D.

Invention, Growth, and Welfare. A Theoretical Treatment of Technological Change, Cambridge, Mass. 1969

Norsworthy, J.R., Harper, M.J., Kunze, $\boldsymbol{K}$.

The Slowdown in Productivity Growth: Analysis of Some Contributing Factors, in: Brookings Papers on Economic Activity $2(1979)$, S. 387-421

O'Driscol1, G.P.jr

Economics as a Coordination Problem, Ransas City 1977

O'Driscoll, G.P. jr, Rizzo, M.J.

The Economics of Time and Ignorance, Oxford und New York 1985

OECD

Positive Anpassungspolitik - strukturanpassung durch Marktwirtschaft, Bundesministerium für Wirtschaft (Hrsg.), Bonn 1984

Olson, $M$.

Stagflation and the Political Economy of the Decline in Productivity, in: American Economic Review, Papers and Proceedings, $72(1982)$, S. 143-148

Paulenz, $R$.

Der Einsatz finanzpolitischer Instrumente in der Forschungsund Entwicklungspolitik, Frankfurt a.M. u.a.0. 1978

Penner, R.G.

A Note on Portfolio selection and Taxation, in: Review of Economic Studies $31(1964)$, S.83-86

Pratt, J.W.

Risk Aversion in the Small and in the Large, in: Econometrica $32(1964)$, S. 122-136 
Presse- und Informationsamt der Bundesregierung

Steuerreform 1986/88 und 1990, Aktuelle Beiträge zur Wirtschafts- und Finanzpolitik Nr. 15/1987, Bonn 1987

Reich, R.B.

An Industrial Policy of the Right, in: Public Interest $73(1983)$, S. 3-17

Reinboth, $H$.

Schuldscheindarlehen, in: Handwörterbuch der Finanzwirtschaft, Stuttgart 1976, Sp. 1594-1603

Reinganum, J.F.

Dynamic Games of Innovation, in: Journal of Economic Theory $25(1981)$, S. 21-41

Reinganum, J.F.

A Dynamic Game of R\&D: Patent Protection and Competitive Behavior, in: Econometrica 50(1982), S. 671-688

Reinganum, J.F.

Uncertain Innovation and the Persistence of Monopoly, in: American Economic Review 73(1983), S. 741-748

Reinganum, J.F.

Practical Implications of Game Theoretic Models of R\&D, in: American Economic Review 74(1984), S. 61-66

Reinganum, J.F.

Innovation and Industry Evolution, in: Quarterly Journal of Economics $100(1985)$, S. 81-99

Reul, $J$.

Erfahrungen mit gesellschafts- und kapitalmarktrechtlichen Maßnahmen zur Verbesserung der Eigenkapitalausstattung der Unternehmen in Frankreich, in: Zeitschrift für Unternehmensund Gesellschaftsrecht 15(1986), S. 70-105

Richter, M.R.

Cardinal Utility, Portfolio selection and Taxation, in: Review of Economic studies 27(1960), S. 152-166 
Richter, W.F.

Modelltheoretische Analyse der steuertarifreform 1986/88 und ihrer dämpfenden Wirkung auf die Risikobereitschaft im mittleren Einkommensbereich, in: Zeitschrift für betriebswirtschaftliche Forschung 37(1985), S. 1070-1077

Richter, W.F., Hampe, J.F.

Measuring the Gain from splitting under Income Taxation, in: Methods of Operations Research 51(1984), S. 385-400

Rōpke, $J$.

Staatsversagen als Ursache der Innovationsschwäche in westlichen Industrieländern, in: Innovationsprobleme in ost und West, A. Schüller, H. Leipold, H. Hamel (Hrsg.), stuttgart und New York 1983, S. 91-114

Rose, G.

Verunsicherte steuerpraxis, in: Steuerberater-Jahrbuch $27(1975 / 76)$, S. $41-84$

Rose, G.

Besteuerung nach Wahl - Probleme aus der Existenz steuerlicher Rechtswahlmöglichkeiten, Grundsätze für ihre Ausnutzung, in: Steuerberater-Jahrbuch $31(1979 / 80)$, S. 49-96

Rose, $G$.

Betriebswirtschaftlich bedeutsame Eigenschaften des Steuersystems, in: Staatsfinanzierung im Wandel, R.-H. Hansmeyer (Hrsg.), Berlin 1983, S. 81-108

Rose, $\boldsymbol{K}$.

Produktivität, in: Handwörterbuch der Sozialwissenschaften, Band 8, Stuttgart und Tübingen 1964, S. 613-619

Rosegger, $G$.

The Economics of Production and Innovation, Oxford u.a.o. 1980

Rother, $D$.

Strukturwandel im Weltschiffbau - Auswirkungen auf die westeuropäische Schiffbauindustrie, dargestellt an den Beispielen der Schiffbauindustrie der Bundesrepublik, Japans und Schwedens, in: Beihefte der Ronjunkturpolitik 31(1985), S. 131-154 


\section{Sachverstāndigenrat}

zur Begutachtung der gesamtwirtschaftlichen Entwicklung, Jahresgutachten 1984/85, Mainz 1984

\section{Sachverstāndigenrat}

zur Begutachtung der gesamtwirtschaftlichen Entwicklung, Jahresgutachten 1985/86, Stuttgart und Mainz 1985

\section{Sachverständigenrat}

zur Begutachtung der gesamtwirtschaftlichen Entwicklung, Jahresgutachten 1986/87, Stuttgart und Mainz 1986

Sandmo, A.

Capital Risk, Consumption, and Portfolio Choice, in: Econometrica.37(1969), s. 586-599

Sandmo, A.

Investment Incentives and the Corporate Income Tax, in: Journal of Political Economy $82(1974)$, S. 287-302

Sandmo, A.

The Effects of Taxation on Savings and Risk-Taking, in: Handbook of Public Economics, Bd. 1, A.J. Auerbach und M.S. Feldstein (Hrsg.), Amsterdam U.a.0. (1985), S. 265-311

Sato, R., Suzawa, G.S.

Research and Productivity, Endogeneous Technical Change, Boston, Mass. 1983

Scherer, F.M.

Research and Development Resource Allocation under Rivalry, in: Quarterly Journal of Economics 81(1967), S. 359-394

Scherer, F.M.

Industrial Market structure and Economic Performance, 2 . Aufl., Chicago 1980

Scherer, F.M.

Time-Cost Trade-offs in Uncertain Empirical Research Projects, in: Naval Research Logistics Quarterly 13(1966), S. 71-82, wiederabgedruckt in: F.M. Scherer, Innovation and Growth, Cambridge, Mass. 1983, S. 67-82 
Schleicher, $\boldsymbol{H}$.

Bestimmungsgründe der sektoralen Produktivitätsentwicklung, Göttingen 1984

Schmalholz, H., Scholz, L.

Innovationsdynamik der deutschen Industrie in den achtziger Jahren, in: ifo-Schnelldienst 40 (1987), S. 20-28

Schmidt, $\boldsymbol{K} .-D$.

Industriepolitik - eine strategie zur oberwindung der strukturkrise?, in: Beihefte der Konjunkturpolitik 31(1985), S. 63-75

Schmidt, R.H.

Ein neo-institutionalistischer Ansatz der Finanzierungstheorie, in: Unternehmensführung aus finanz- und bankwirtschaftlicher sicht, E. Rühli und J.-P. Thommen (Hrsg.), stuttgart 1981, S. 135-154

Schmidt, R.H.

Venture Capital aus der Sicht der Finanzierungstheorie, in: Betriebswirtschaftiche Forschung und Praxis 37(1985), S. 421-437

Schmidt, $H$.

Freiverkehrsmärkte an Europas Börsen, in: Die Bank 27 (1987), S. $288-298$

Schmookler, $J$.

Invention and Economic Growth, Cambridge, Mass. 1966

Schneider, $D$.

Gewinnbesteuerung und Risikobereitschaft: zur Bewährung quantitativer Ansätze in der Entscheidungstheorie, in: Zeitschrift für betriebswirtschaftliche Forschung 29(1977), S. $633-666$

Schneider, $D$.

Investition und Finanzierung, 5. Aufl., Wiesbaden 1980

Schneider, $D$.

Offentliche Einnahmen und Unternehmensrisiko, in: Staatsfinanzierung im Wandel, R.-H. Hansmeyer (Hrsg.), Berlin 1983, S. 63-79 
Schrumpf, $H$.

Rleine und mittlere Unternehmen in der technologiepolitischen Konzeption der Bundesregierung, in: RWI-Mitteilungen $37 / 38(1986 / 87)$, S. $239-256$

Schürmann, H., Beyer, G.

Umwandlung in die Aktiengesellschaft - Steuerliche Nachteile?, in: Zeitschrift für Unternehmens- und Gesellschaftsrecht $10(1981)$, S. 58-100

Schumpeter, J.A.

Business Cycles, Bd. 1, New York 1939

Schumpeter, J.A.

Ronjunkturzyklen, Bd. 1, Göttingen 1961

Schumpeter, J.A.

Theorie der wirtschaftlichen Entwicklung, 6. Auflage, Berlin 1964

Schwark, $\boldsymbol{B}$.

Das neue Kapitalmarktrecht, in: Neue Juristische Wochenschrift $40(1987)$, S. 2041-2048

Seelbach, H.

Die Thesen von Modigliani und Miller unter Berücksichtigung von Ertrag- und Substanzsteuern, in: Zeitschrift für Betriebswirtschaft $49(1979)$, S. 692-709

sinn, H. $-W$.

Systeme der Kapitaleinkommensbesteuerung - Ein allokationstheoretischer Vergleich, in: Beiträge zur neueren steuertheorie, D. Bös, M. Rose und Ch. Seidl (Hrsg.), Berlin u.a.o. 1984, S. 209-238

Sinn, H. $-W$.

Rapitaleinkommensbesteuerung. Eine Analyse der intertemporalen, internationalen und intersektoralen Allokationswirkungen, Tübingen 1985

Sohmen, $E$.

Allokationstheorie und Wirtschaftspolitik, Tübingen 1976 
Spence, A.M.

Cost Reduction, Competition, and Industry Performance, in: Econometrica 52(1984), S. 101-121

Stackelberg, H. von

Möglichkeiten und Grenzen der Wirtschaftslenkung, in: ORDO $2(1949)$, S. 193-206

\section{Statistisches Bundesamt}

Fachserie 18, Reihe S. 7, Lange Reihen 1950-1984, Wiesbaden 1985

Staudt, $E$.

Der technologiepolitische Aktivismus in der Bundesrepublik, in: Wirtschaftsdienst 65(1985), S. 464-470

Staudt, $B$.

Technologie- und Regionalpolitik der Länder: Vom Leistungszum Subventionswettbewerb, in: List-Forum 14(1987/88), S. 93-110

stiglitz, J.E.

The Effects of Income, Wealth, and Capital Gains Taxation on Risk-Taking, in: Quarterly Journal of Economics 83(1969), S. $263-283$

stiglitz, J.E.

Taxation, Risk Taking, and the Allocation of Investment in a Competitive Economy, in: Studies in the Theory of Capital Markets, M.C. Jensen (Hrsg.), New York u.a.O. 1972, S. 294-374

stoltenberg, $G$.

Einbringungsrede des Bundesministers der Finanzen zum Haushaltsgesetz 1988, in: Bulletin, Presse- und Informationsamt der Bundesregierung (Hrsg.), Nr. 84, Bonn 1987, S. 721-731

streit, M.E.

Innovationspolitik zwischen Unwissenheit und Anmaßung von Wissen, in: Hamburger Jahrbuch für Wirtschafts- und Gesellschaftspolitik $29(1984)$, S. 35-54 
Swoboda, P., Zechner, $J$.

Unternehmensbesteuerung und Risikokapitalbildung, in: Betriebswirtschaftiche Forschung und Praxis 37(1985), S. $403-420$

Teece, D.J.

Economies of Scope and the scope of Enterprise, in: Journal of Economic Behavior and Organization 1(1980), S. 223-247

Tenhof, $D$.

Diskussionsbeitrag, in: Mehr soziale Marktwirtschaft - weniger Subventionswirtschaft, Ludwig-Erhard-stiftung e.v. Bonn (Hrsg.), Stuttgart und New York 1984, S. 115-118

Tobin, $J$.

Liquidity Preference as a Behavior Towards Risk, in: Review of Economic studies 25(1958), S. 65-86

Tollison, R.D.

Rent Seeking: A Survey, in: Kyklos 35(1982), S. 575-602

Tullock, G.

The Transitional Gains Trap, in: Bell Journal of Economics $6(1975)$, S. 671-678

Tullock, G.

The Cost of Transfers, in: Toward a Theory of the Rent-Seeking Society, J.M. Buchanan, R.D. Tollison und G. Tullock (Hrsg.), College station 1980, S. 269-282

Turvey, $R$.

On Divergences between Social Cost and Private Cost, in: Economica $30(1963)$, S. 309-313

U.S. Bureau of the Census

Statistical Abstract of the United States, verschiedene Auflagen, Washington D.C.

Usher, D.

The Welfare Economics of Invention, in: Econometrica $31(1964)$, S. 279-287 
Utterback, J.M.

Recent Findings and Hypotheses about the Dynamics of Product and Process Change, in: Innovation, Economic Change and Technology Policies, R.A. Stroetmann (Hrsg.), Basel und stuttgart 1977 , s. 7-20

Utterback, J.M.

The Dynamics of Product and Process Innovation in Industry, in: Technological Innovation for a Dynamic Economy, C.T. Hill und J.M. Utterback (Hrsg.), New York U.a.0. 1979a, S. 40-65

Utterback, J.M.

Product and Process Innovation in a Changing Competitive Environment, in: Industrial Innovation, M.J. Baker (Hrsg.), London und Basingstoke 1979b, S. 135-147

Van Cayseele, $P$.

Economies of Scope in Research and Development, in: Journal of Economics $47(1987)$, S. 273-285

Vollmer, $L$.

Der Genußschein - ein Instrument für mittelständische Unternehmen zur Eigenkapitalbeschaffung an der Börse, in: Zeitschrift für Unternehmens- und Gesellschaftsrecht 12(1983), S. 445-475

Vollmer, $L$.

Eigenkapitalbeschaffung für die $\mathrm{GmbH}$ durch Börsenzugang, in: GmbH-Rundschau $75(1984)$ ， S. 329-339

Wagner, F.W., Dirrigl, H.

Die Steuerplanung der Unternehmung, stuttgart und New York 1980

Wagner, $\boldsymbol{K}$.

Competition and the Productivity: A study of the Metal Can Industry in Britain, Germany and the United States, in: Journal of Industrial Economics 29(1980), S. 17-35

Weichert, $R$.

Mehr Risikokapital durch Erleichterung des Börsenzugangs für Unternehmen, Diskussionsbeitrag, Kiel 1985 
Weisskopf, T.B., Bowles, S., Gordon, D.M.

Hearts and Minds: A Social Model of U.S. Productivity Growth, in: Brookings Papers on Economic Activity 2(1983), S. 381-441

Werner, W., Kindermann, $\boldsymbol{E}$.

Umwandlung mittelständischer Unternehmen in eine Aktiengesellschaft: Gesellschaftsrechtliche Vor- und Nachteile und Verfahren, in: Zeitschrift für Unternehmens- und Gesellschaftsrecht $10(1981)$, S. 17-57

White, L.J.

What Has Been Happening to the Aggregate Concentration in the United States?, in: Journal of Industrial Economics 29(1981), S. $223-230$

Wilhelm, $J$.

Die Bereitschaft der Banken zur Risikoübernahme im Kreditgeschäft, Diskussionspapier, Bonn 1982

Wilson, J.H.

Microeconomics: Concepts and Applications, New York 1981

Wirtschaftswoche

Forschungsförderung: Mittelstand im Abseits, Nr. 32, $31.7 .1987 a$, S. 16-20

Wirtschaftswoche

Technologiezentren: Erste Auslaufmodelle, Nr. 32, 31.7.1987b, S. 48-50

Wiss. Beirat beim Bundesminister für Wirtschaft

Wettbewerbspolitik, Gutachten, Bonn 1986

Wiss. Beirat beim Bundesministerium der Finanzen

Stellungnahme zur Tarifstruktur der körperschaftsteuer, Bonn 1987

Wōhe, G., Bilstein, J.

Grundzüge der Unternehmensfinanzierung, 4. Aufl., München 1986 
Zajac, E.E.

A Geometric Treatment of Averch/Johnson's Behavior of the Firm Model, in: American Economic Review 60(1970), S. 117-125

Zechner, $J$.

Managerverhalten und die optimale Kapitalstruktur von Unternehmungen, in: Journal für Betriebswirtschaft 32(1982), . 180-197

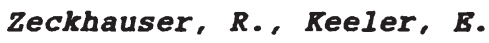

Another Type of Risk Aversion, in: Econometrica 38(1970), S. $661-665$

Zeppernick, $\boldsymbol{R}$.

Mehr staat oder mehr Markt?, in: Hamburger Jahrbuch für Wirtschafts- und Gesellschaftspolitik 30(1985), S. 69-84

Zeppernick, $\boldsymbol{R}$.

Zur Rolle des staates in der Sozialen Marktwirtschaft, Tübingen 1987 
Friedhelm Herb - 978-3-631-75475-7 Downloaded from PubFactory at 01/11/2019 04:33:39AM via free access 


\section{HOHENHEIMER VOLKSWIRTSCHAFTLICHE SCHRIFTEN}

Band 1 Walter Deffaa: Anonymisierte Befragungen mit zufallsverschlüsselten Antworten. Die Randomized-Response-Technik (RRT). Methodische Grundlagen, Modelle und Anwendungen. 1982.

Band 2 Thomas Michael Baum: Staatsverschuldung und Stabilisierungspolitik in der Demokratie. Zur neoinstitutionalistischen Kritik der keynesianischen Fiskalpolitik. 1982.

Band 3 Klaus Schröter: Die wettbewerbspolitische Behandlung der leitungsgebundenen Energiewirtschaft. Dargestellt am Beispiel der Fernwărmewirtschaft der Bundesrepublik Deutschland. 1986.

Band 4 Hugo Mann: Theorie und Politik der Steuerreform in der Demokratie. 1987.

Band 5 Max Christoph Wewel: Intervallarithmetische Dependenzanalyse in der Ökonometrie. Ein konjekturaler Ansatz. 1987.

Band 6 Heinrich Pascher: Die U.S.-amerikanische Deregulation Policy im Luftverkehrs- und Bankenbereich. 1987.

Band 7 Harald Lob: Die Entwicklung der franzósischen Wettbewerbspolitik bis zur Verordnung Nr. 86-1243 vom 01. Dezember 1986. Eine exemplarische Untersuchung der Erfassung der Behinderungsstrategie auf der Grundlage des Konzepts eines wirksamen Wettbewerbs. 1988.

Band 8 Ulrich Kirschner: Die Erfassung der Nachfragemacht von Handelsuntemehmen. Eine Analyse der okonomischen Beurteilungskriterien und der wettbewerbsrechtlichen Instrumente im Bereich der Verhaltenskontrolle.1988.

Band 9 Friedhelm Herb: Marktwirtschaftliche Innovationspolitik. 1988 
Friedhelm Herb - 978-3-631-75475-7 Downloaded from PubFactory at 01/11/2019 04:33:39AM via free access 
Friedhelm Herb - 978-3-631-75475-7 Downloaded from PubFactory at 01/11/2019 04:33:39AM via free access 
Friedhelm Herb - 978-3-631-75475-7 Downloaded from PubFactory at 01/11/2019 04:33:39AM via free access 